
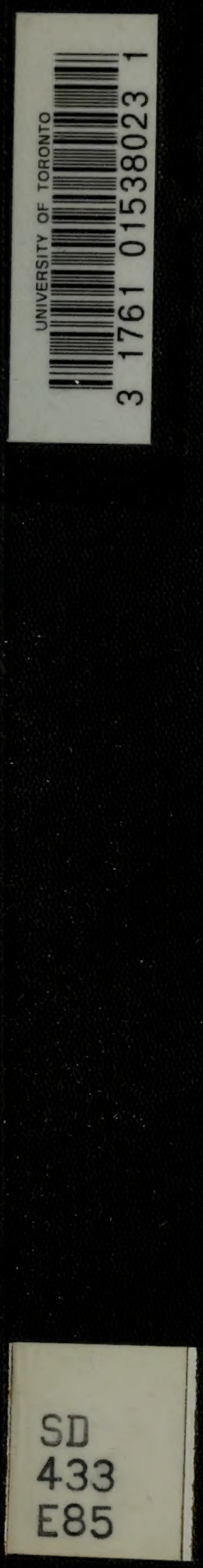


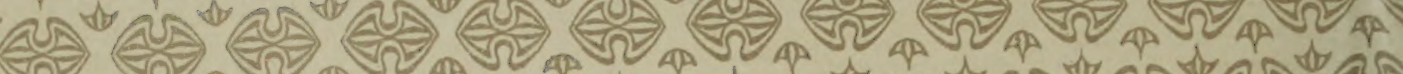





\section{DIE MECHANISCHE}

\section{TECHNOLOGIE DES HOLZES.}

ZUNÄCHST FÜR

TECHNISCHE UND FORSTLICHE HOCHSCHULEN.

VoN

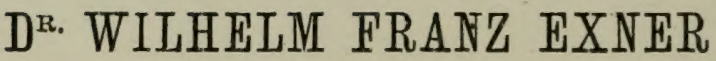

o. Ö. PROFESSOR DER INGENIEUR - WISSENSCHAFTEN AN DER K. K. FORST-AKADEMIE MARIABRUNN.

MIT VIELEN TAFELN UND HOLZSCHNITTEN.

I. BAND.

DIE MECHANISCHEN EIGENSCHAFTEN DES HOLZES.

( I. HÄLFTE.)

\section{WIEN, 1871.}

W I L H E L M B R A U M Ü L L E R K. K. HOF- UND UNIVERSITÄTSBUCHHÄNDLER. 


\section{DIE MECHANISCHEN}

\section{EIGENSCHAFTEN DES HOLZES.}

EINE ABHANDLUNG

VORGELEGT

DER AKADEMIE DER WISSENSCHAFTEN IN PARIS

VON

E. CHEVANDIER UND G. WERTHEIM.

REVIDIRT UND ÜBERSETZT

VON

PROF. DR. WILHELM FRANZ EXNER.

I. HÄLFTE.

\section{LIBRARY NIT zwEL TaRLLX \\ FACULTY OF FORESTRY UNIVERSITY OF TORONTO}

WIEN, $18 \% 1$.

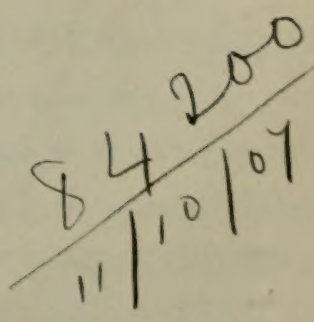

W I L H E L M B R A U M Ü L L E R K. K. HOF- UND UNIVERSTTÄTSBUCHHÄNDLER. 


$$
\begin{aligned}
& S D \\
& 433 \\
& E 85
\end{aligned}
$$




\section{VORWOR T.}

Die vorliegende Uebersetzung der Arbeit Chevandier's und Wertheim's über die mechanische Beschaffenheit des Holzes bildet gleichsam die Einleitung zu jenem Werke über mechanische Technologie des Holzes, dessen Zusammenstellung sich der Unterzeichnete zur Aufgabe gemacht hat.

Je kostbarer das Holz wird, ohne dabei entbehrlicher zu werden, desto wichtiger ist die Kenntniss dieses Rohstoffes, die Popularisirung desjenigen, was die Wissenschaft über die Natur desselben erforscht hat.

Aber auch die Bearbeitung des Holzes und die zu derselben nöthigen Vorrichtungen sind bisher nicht vollständig behandelt worden.

Endlich dürfte eine Darstellung der Holz verarbeitenden Gewerbe nach ihrem heutigen Stande gewiss kein überflüssiges Unternehmen sein.

Die Besprechung der Eigenschaften des Holzes, die Darstellung der Bearbeitung desselben und die Beschreibung der einzelnen Gewerbe bilden die drei Hauptabtheilungen der „Technologie des Holzes", welche im Laufe der nächsten Jahre erscheinen soll.

Warum der Verfasser gerade von der Arbeit Chevandier's und Wertheim's ausging, lässt sich kurz auseinandersetzen.

Diese Abhandlung ist nämlich das beste und vollständigste, was in der Erforschung der mechanischen Eigenschaften geleistet worden ist; dieselbe berïcksichtigt aber alle vorangegangenen Arbeiten und es erübrigt nur noch, die nach dem Jahre 1850 in dieser Beziehung gesammelten Ergebnisse und Erfahrungen geordnet wiederzugeben, was in der zweiten Hälfte des ersten Bandes geschehen wird. 
Die Uebersetzung der oft genannten Arbeit, welche die Grundlage für die Feststellung der meisten im praktischen Ingenieurwesen für das $\mathrm{Holz}$ gebräuchlichen Ziffern abgab, war nach dem Erachten vieler unserer Freunde Bedürfniss.

Der Zweck, den die Herren $\mathrm{Chevandier}$ und Wertheim vor Augen hatten, ist, die mechanischen Eigenschaften der einheimischen (französischen) Holzarten sowohl in theoretischer als praktischer Hinsicht zu studiren. Es wurden dabei in successiver Aufeinanderfolge die allgemeinen Gesetze, die Grenzen derselben bei den Individuen und die Abweichungen, welche den Unterschieden der Art, des Alters, der Exposition und der Herkunft zugeschrieben werden müssen, gesucht.

Die Abhandlung zerfällt in fünf Theile: Die Geschichte der Frage, die Beschreibung der Vorrichtungen und das Detail der Versuche, der Calcul der Versuche und die Besprechung der angewendeten Versuchsmethoden, Discussion der gefundenen Resultate und endlich die Folgerungen. 


\section{ERSTER THEIL.}

\section{Geschichtliches.}

Die ersten Autoren, welche die mechanische Beschaffenheit der Stoffe untersuchten, haben sich nur mit der Festigkeit (Cohäsion) und dem specifischen Gewichte (Dichte, Densité), sowie mit dem Zusammenhang dieser beiden Eigenschaften beschäftiget. Die genaue Untersuchung der Elasticität ist erst später, und zwar erst nach der Einführung des Begriffes des Elasticitätscoëfficienten in die Wissensehaft durch Young und Tredgold, gefolgt.

Was nun die Hölzer betrifft, so sind die hauptsächlichsten Ansichten der Forscher über die wesentlichsten Punkte so sehr von einander abweichend, dass wir nichts besseres thun können, um eine klare Vorstellung davon zu geben, als, diese Ansichten wörtlich zu citiren und die numerischen Resultate für die Dichte, Festigkeit und den Elasticitätscoëfficienten, zu welchen sie führten, in einer Zusammenstellung zu vereinigen. (Siehe Tabelle Nr. I.) *)

Parent (Expériences sur la résistance des bois de chêne et de sapin, Memoiren der Pariser Akademie der Wissenschaften 1707 u. 1708, S. 516) findet: dass die mittlere Festigkeit („Force“) der Tanne zu jener der Eiche sich verhält beilïutig wie $355 \mathrm{zu}$ 300 oder wie $119 \mathrm{zu} 100$.

Es ist wohl zu bemerken, dass die Mehrzahl der Parent'schen Versuche mit sehr schwachen Eichen angestellt wurden.

*) Es werden in dieser Tabelle nur jene Autoren citirt, welche sich speciell mit den mechanischen Eigenschaften des Holzes befast haben. 
Musschenbroeck (Introductio ad philosophiam naturalem, Lugduni Batavorum 1762, I. Band, S. 409):

„Der Theil der Bäume, wolcher gegen Norden gerichtet ist, ist in der Mehrzahl der Fälle jedoch nicht immer von schmäleren Jahrringen gebildet, die Kälte des Nordens hindert nämlich das Fortschreiten und die Entwickelung der Vegetation; die dem Süden zugewendete Seite setzt sich dagegen aus grösseren Jahrringen zusammen, aber ich habe auch manchmal das Gegentheil gesehen ....."

"Bei allen meinen Versuchen habe ich die folgenden Kesultate gewonnen: Die Festigkeit des Herzens des Baumes ist die schwächste.....Vom Kern ausgehend ist die Festigkeit im ganzen südlich gelegenen Theile grösser, als in dem gegen Norden exponirten; die Festigkeit im westlichen Theil ist ein Mittel zwischen den beiden ersten Festigkeiten, jene des östlichen zeigt sich aber als die grösste. Wenn man weiters das Holz von der Axe bis zur Peripherie in diesen vier Richtungen verfolgt, so findet man das festeste $\mathrm{Holz}$ an einer mittleren Stelle, die zwischen Rinde und Mark liegt, und die dem Centrum zunächst gelegenen Theile werden um ein Bedeutendes von jenen in der Nähe des Splintes an Festigkeit übertroffen."

"Die Festigkeit der höheren Theile des Stammes, wo die Aeste ausgehen, differirt von jener der dem Boden benachbarten fast nicht, auch gibt es keine derartigen Unterschiede zwischen Stamm und Aesten. Ich weiss, dass mehrere Physiker einer entgegengesetzten Ansicht sind; sie behaupten, der Kern des Baumes enthalte das härteste und festeste Holz, und in gleicher Entfernung rund um die Axe sei es von gleicher, aber schwächerer Cohäsion, der Splint endlich sei die schwächste Partie, ich aber führe einfach das an, was mich die Versuche mit unseren Bäumen gelehrt haben."

"Es gibt eine von der Natur des Bodens herrührende Differenz. Die Bäume, welche auf einem sandigen Boden erwachsen, sind gebrechlicher, sowie die auf einem thonichten Grunde stehenden, zïher sind. Das frischgeschnittene und noch feuchte Holz ist stärker, als das gleiche getrocknete."

Im Allgemeinen basirt die Arbeit Musschenbroeck's, obwohl sie, beconders was die Variationen der Festigkeiten in eincm und demselben Baume betrifft, eine der vollstïndigsten ist, nicht auf einer himreichenden Zahl geniigend ïberzengender Versuche, um die angeführten Folgerungen ganz zu rechtfertigen. - Er*selbst fügt auch später hinzu: "Vielleicht habe ich 


\section{$-3-$}

nicht alle Umstïnde beachtet, welche auf die Festigkeit der IIölzer Einfluss nehmen."

Buffon (Oeuvres de Buffon. 1. Band, S. 10):

„Das junge Holz ist weniger stark, als das ältere: ein dem Fusse des Baumes entnommener Barren hält mehr, als ein vom Gipfel kommender, ans. Ein von dem Umfang, nahe dem Splint, des Baumes herrührendes Stück, ist weniger fest, als ein gleiches vom Mittelpunkt."

"Ausserdem modificirt der Grad der Trockenheit sehr dessen Widerstandsfähigkeit; das grüne Holz bricht viel schwerer als trockenes."

Seite 18: „Das Holz, welches auf einem gewissen Boden am schnellsten erwächst, ist das stärkste, dasjenige, welches langsam erwachsen ist, und bei dem die Jahrringe, das sind die Holzschichten, sehr klein sind, ist minder fest als ersteres."

"Ich habe gefunden, dass die Festigkeit des Holzes seinem Gewichte proportional ist, folglich dass ein Stück, welches mit einem andern gleiche Länge und Dicke hat, aber schwerer ist, auch beiläufig in demselben Verhältnisse fester sein wird."

Seite 27: „Die Dichte des Holzes nimmt vom Centrum gegen den äussersten Umfang des Splintes hin nach einer arithmetischen Progression ab...."

"Das Holz vom Fuss des Baumes wiegt mehr, als jenes vom Stamm in der Mitte seiner Höhe, und dieses wieder wiegt mehr, als jenes vom Gipfel, und zwar nahezu nach einer arithmetischen Progression, welche von dem Wachsthum des Baumes abhängt. Es gibt eine Zeit, zu welcher das Holz des Centrums und der Peripherie des Kernes gleiches Gewicht haben, und das ist die Zeit, in der das Holz in seiner Vollkommenheit ist, (diese Erfahrung ist an Büumen von 40-46 Jahren gemacht worden); aber bei 100 bis 110 jährigen Bäumen war der Kern nicht mehr der solideste Theil des Baumes; - der Splint ist schwerer und fester in alten, als in jungen Bäumen."

Es ist wohl zu beachten, dass die Arbeit Buffon's, obwohl sie nach einem sehr grossen Maassstabe durchgeführt wurde, sich nur auf Eichenholz stützte, was also nicht gestatten würde, seine Schlüsse, selbst wenn sie alle erwiesen wären, auf andere Arten anzuwenden.

Duhamel du Monceau (Traité de la conservation et de la force des bois. 1780).

Seite 50: „Man soll trockene Hölzer anwenden....." 
Seite 56: „Aber das Holz bedarf einer kleinen Quantität Feuchtigkeit, damit es hart sei, woraus ich schliesse, dass die zu trockenen Hölzer keine guten Dienste leisten können."

Stite 65: „Das Holz, das man vom Fusse des Baumes nimmt, ist schwerer, als jenes vom Gipfel."

Seite 71: „Das grüne Holz muss ein Drittheil seines Totalgewichtes verlieren, um für so trocken zu gelten, dass es dieselbe Wirkung habe, wie ein Hygrometer."

Seite 264: „Es scheint, dass die Extraction des Saftes der Hölzer sie nichts von ihrer Festigkeit verlieren macht, nachdem der Saft auch nicht die Festigkeit, welche von der Zahl und Stärke der Fasern abhängt, vermehren kann. Der Saft macht die Holzfiber geschmeidiger und geneigter zu zerreissen."

Seite 378: „Das ist noch eine erwiesene Thatsache, dass ihre Jahrringe (die Jahrringe von Mastbäumen ausgezeichneter Beschaffenheit, die in einem sehr kalten Lande erwachsen sind) näher an einander und kleiner sind."

Seite 411: „So lange die Bäume kräftig und im lebhaften Wachsthum begriffen sind, ist das Kernholz das festeste; bei starken Bäumen, welche in die Ringbildung eintreten, ist das Kernholz oft leichter als das Holz des Ringes zwischen Kern und Umfang; so dass das Holz nach und nach an Dichte gewinnt, und wenn es die höchste Ziffer erreicht hat, wieder nach und nach an Dichte verliert."

Seite 458: "Die Bodengattungen, welche am geeignetsten sind, die schönsten Bäume hervorzubringen, sind nicht diejenigen, welche die Bäume bester Qualität geben."

Seite 458: „In diesen starken Fichten (Pin du Nord von beiläufig 260 Jahren) ist das festeste $\mathrm{Holz}$ dasjenige, welches sich im fünften Ring, vom Centrum aus, befindet, wenn man die Querschnittsfläche, inclusive Splint in 6 gleich breite Ringe theilt; aber man begreift, dass das ein Gegenstand ist, der sehr von den Umständen verändert werden kann."

Die drei Autoren, von denen wir oben Auszïge gegeben haben, sind fast die einzigen, welche sich mit den in einem und demselben Baume vorkommenden Unterschieden der Dichte und Festigkeit und mit dem Einfluss des Bodens beschäftigt haben. Die Divergenz ihrer Ansichten hat diese grossen Fragen unentschieden gelassen. Sie ist vielleicht theilweise der geringen Gleichförmigkeit und Genauigkeit, deren die Bruchversuche fähig sind, zuzuschreiben. 
Wir übergehen mit Stillschweigen die Untersuchungen, welche Duhamel uiber den Einfluss der Risse und über jenen anstellte, den die Beziehungen zwischen der Zusammendrïckung und Verlängerung der Fasern auf den Totalwiderstand von der Biegung unterworfenen Stiicken ausüben.

Die späteren Autoren haben sich besonders mit dem Studium der Elasticität der Hölzer abgegeben.

Girard (Traité de la résistance des solides 1798. S. 183) in diesem Punkt mit Perronet (Oeuvres de Perronnet 1782. Band I. Abhandlung über die Pfähle und Piloten. S. 93) übereinstimmend schliesst aus dem Gange seiner Versuche, dass sich die Elasticität der Eiche zu jener der Tanne wie 63 zu 47 verhält; er sagt endlich (S. 39), dass die Fortdauer der Einwirkung der Last die Pfeilhöhe der Ausbiegungscurve vergrössert, was, nach seiner Ansicht, nie statthaben kann, wenn die Elasticität sich nicht verändert und in jedem Augenblick einen gewissen Theil seiner Kraft verliert.

Belidor (Architecture hydraulique 1782), Rondelet (Art de bâtir 1814), Barlow (Essay on the strength of timber 1817), Ebbels und Tredgold in verschiedenen Werken des letzteren und besonders in "l'art du charpentier" haben die Dichte, die Festigkeit und den Elasticitätscoëfficienten für eine grosse Anzahl von Holzarten verschiedener Herkunft bestimmt.

Charles Dupin (Expériences sur la flexibilité, la force et l'élasticité im Journal der École polytechnique. X. Band, 1815) hat eine grosse Arbeit über die mechanischen Eigenschaften des Holzes veröffentlicht. Dupin untersuchte die Natur der elastischen Curve, die Lage der neutralen Schichte (unveränderliche Faser); er berichtigte die Formeln, welche die Beziehungen zwischen den Dimensionen des Stückes dem angewendeten Gewichte und der erzeugten Ausbiegung ausdrückten.

Seite 142. Er beweist, dass: „Die Krümmungen der Hölzer, welche durch sehr kleine Gewichte hervorgebracht werden, diesen Gewichten proportionirt sind", und Seite 150 folgert er aus einem die Eiche, die Cypresse, die Rothbuche und die Tanne enthaltenden Tablean: „Dass die specifischen Gewichte gleichzeitig, aber in viel geringerem Grade mit den Widerständen gegen die Biegung wachsen."

Seite 194. Dupin bemerkt: „Dass die Kräfte, die man anwenden muss, um die Hölzer zum Bruch zu bringen, keine nothwendige Beziehung mit den die Ausbiegung der Hölzer hervorrufenden Kräften haben." 
"So setzen einige Pflanzenarten der Biegung einen sehr geringen Widerstand, dem Bruch einen grossen entgegen; solche sind die Rothbuche, der Nussbaum, die Ulme, die Tanne etc. Einige Arten widerstehen im Gegentheil sehr stark der Biegung und verhältnissmässig viel weniger dem Bruche, z. B. die Cypresse, das Acajou etc. Andere endlich bieten gleichzeitig grossen Widerstand der Biegung und dem Bruche dar, hieher gehören die corsische Fichte und die Eiche."

Diese Eintheilung führt Dupin dazu, die beste Anwendung dieser verschiedenen Holzarten in der Praxis anzugeben.

Bevan (Philosophical Transactions, 1829) hat sich besonders mit der Bestimmung des Moduls der Elasticität durch Torsion befasst.

Savart (Mémoires de l'Academie des Sciences, 1830) hat sich der durch Tonschwingungen auf Platten erzeugten Knotenlinien bedient (welche Platten aus Rothbuchenholz nach verschiedenen Richtungen herausgeschnitten worden waren), um die Unterschiede und die Richtung der Axen der Elasticität zu ermitteln.

Er bemerkt Seite 404: „dass in den Hölzern, wo die Jahresschichten nahezu cylindrisch und concentrisch sind, die Elasticität nach allen Radien in jedem beliebigen zur Axe senkrechten Schnitt auffallend gleichförmig ist."

Seite 407: "Jeder Stab kann bei derselben Art der Eintheilung, je nachdem die Schwingung nach der Breite oder Dicke Platz greift, zwei Töne zum Vorschein bringen, aber man kann diesen Unterschied, wenn jene Abmessungen sehr klein sind, leicht vernachlässigen. ${ }^{*}$

Savart nimmt drei Axen an: „die erste, parallel zu den Fasern, die zweite im Sinne des Radius und die dritte tangentiell zu den Jahrringen. Er findet durch Versuche, die er mit kleinen Barren im Sinne dieser drei Axen genommen angestellt hat, dass wenn man als Einheit den Widerstand gegen Biegung im Sinne der Tangente, dieser dem Radius folgend 2.25 und in der Richtung der Axe 16 beträgt."

Wheatstone (Philosophical Transactions, 1833, S. 608): "Wenn man cine Holzplatte so vorrichtet, dass die Fibern zu einer der Seitenkanten parallel laufen, so sind die Axen der grössten und kleinsten Elasticität rechtwinkeliģ zu einander und parallel zu den anliegenden Seiten gestellt."

"Wenn die Platte ein rechtwinkeliges Parallelopiped ist, ressen Seitenkanten sich umgekehrt wie die Quadrate ihrer 
Widerstände gegen Biegung verhalten, so werden die beiden Arten der Vibration parallel zu den Seiten, wiewol diese verschieden lang sind, isochronisch sein, und ihre Coëxistenz wird cine resultirende Gestalt, deren Linien parallel zur Diagonale sind, ergeben."

Man könnte also, indem man die diesen Seiten zu gebende relative Länge durch Versuche ermittelt, die Beziehung der Elasticitätscoëfficienten in zwei auf einander senkrechten Richtungen finden.

Poncelet (Mécanique industrielle, 1839. S. 316) geht in sehr genaue Details über die Elasticität der Hölzer und namentlich ïber die Versuche durch Ausdehnung ein. Er macht nach den Versuchen von Minard und Désormes und jenen von Ardant ersichtlich, dass bei den ersten Belastungen die Verlängerungen den spannenden Kräften fühlbar proportional sind, und rechnet nach diesen Verlängerungen die in unserer Tabelle I. enthaltenen Elasticitätscoëfficienten.

Die Elasticitätsgrenze für die Eiche entspricht nach den Versuchen von Minard und Désorme einer Belastung von 2.13 Kil. per Quadrat-Millimeter und einer Verlängerung 0.0016 der ursprünglichen Länge.

Diese Grenze ist für die Tanne der Vogesen, nach den Erfahrungen Ardant's einer Belastung von 1.85 Kil. per QuadratNillimeter und einer Verlängerung von $0 \cdot 00117$ entsprechend.

Diese verschiedenen Resultate bestimmen die Elasticität im Sinne der Länge der Fasern. Poncelet spricht, indem er sie mitheilt, den Wunsch aus, es möchten analoge Versuche iiber den Elasticitätswiderstand im Sinne des Radius und der Tangente der Holzringe gemacht werden.

Nach Eaton Hodgkinson (Combes, Exploitations des mines, I. Band, S. 550) alterirt ganz namhaft eine Verkuirzung von 0.0027 der urspriinglichen Lïnge eines nicht gebogenen Prisma's die Elasticität um ein sehr namhaftes.

Hagen (Poggendorf's Annalen, LVIII. Band, S. 125) hat die Elasticität mehrerer Holzarten durch die Biegung von Stäben, die im Sinne der Fasern und senkrecht auf dieselben genommen waren, untersucht, und hat keine grosse Differenz zwischen Kernund Splintholz gefunden; er hat indessen erkannt, dass der Elasticitätscoëfficient bedeutend abnimmt, wenn das Holz sehr stark durchnässt ist.

Paccinotti und Peri (Il Cimento, 3. Jahrgang, 1845) haben, nachdem wir die vorliegende Arbeit begonnen hatten, umstand- 
liche und präcis durchgeführte Versuche über die Elasticität der Hölzer veröffentlicht. Sie haben das Ziel gehabt, speciell die verschiedenen Methoden, welche man zur Bestimmung des Elasticitätscoëfficienten anwendet, untereinander zu vergleichen und dieselben zu berichtigen; endlich jene Beziehungen zwischen der Dichte und dem Elasticitätscoëfficienten aufzufinden, welche einer uns für die Metalle schon bestimmt hatte.

Diese Physiker haben mit quadratischen Holzstäben von 27 zu 36 Millimeıer Seite, nach den drei Methoden durch Biegung, Verlängerung und Torsion, operirt.

Ihre Versuche für die Biegung sind nach fünf verschiedenen Verfahren angestellt worden.

1. Indem die Enden der Stäbe auf Steinlagern unterstützt wurden.

2. Indem diese Enden auf bronzene um eine horizontale Axe drehbare Isager gelegt wurden.

3. Indem sie auf fixe Lager messingener Cylinder gelegt waren.

4. Indem an den Erden der Stäbe metallische um eine Axe bewegliche Platten angebracht wurden, die ihrerseits durch einen an Ketten aufgehängten Haken getragen wurden.

5. Indem ein Ende fest eingelassen wurde.

Diese Experimentatoren haben sowol die elastischen als permanenten Verlängerungen, die Torsionswinkel und die verschiedenen Punkte des Stabes entsprechenden Ordinaten bei der Biegung desselben bei wachsender Belastung gemessen.

In dem zweiten Theil ihrer Arbeit vergleichen Paccinotti und Peri die ziffermässigen Ergebnisse ihrer Versuche, mit jenen, die sie aus den bekannten Formeln ableiteten und suchen eine Relation zwischen der Dichte und dem Elasticitätscoëfficienten der von ihnen geprüften Hölzer aufzustellen.

Sie gelangen endlich zu folgenden Schlüssen:

1. "Die Elasticität erzeugt in den verschiedenen Theilen des Holzes Veränderungen, welche nicht blos für die ersten $\mathrm{Be}$ lastungen, sondern auch für jene dem Bruche sehr naheliegenden, den angewendeten Spannungen proportional sind, vorausgesetzt dass man Sorge trägt unter dem Maass der Elasticität nicht auch die permanente Aenderung zu begreifen, die entweder der Wcichheit der Substanz oder der Permanenz der Belastung zuzuschreiben ist." 
2. „Die Curven, welche die mit einem Ende fest eingelassenen Hölzer annehmen sind nicht dieselben, wie jene, welche die gleichen Hölzer, sobald sie an beiden Enden unterstïtzt sind, annehmen würden, was man der Gegenwirkung der Fasern in den beiden entgegengesetzten Aesten zuschreiben muss. Indessen kann dieselbe Theorie dazu dienen, um die beiden Arten von Curven abzuleiten vorausgesetzt, dass bei der Integration der Differentialgleichung auf die gehörige Bestimmung der Constanten Rücksicht genommen werde. (Der Werth derselben hängt von dem Grad der Unveränderlichkeit der Einfügung des Endes ab.)"

3. "Die Unterschiede, die sich bei der Bestimmung des Elasticitätscoëfficienten ergeben haben, verschwinden fast vollständig, wenn man mit diesem Ausdrucke $\mathrm{E}^{\prime}={ }_{\mathrm{G}}^{\mathrm{E}}$ bezeichnet, worin $\mathrm{F}$ den bisher allgemein angenommenen Begriff des Elasticitätscoëfficienten und $G$ das specifische Gewicht bedeutet."

4. „Der Elasticitätscoëfficient $\mathrm{E}^{\prime}$ ist, wiewohl es einige Unterschiede unter den diversen Holzarten gäbe, im allgemeinen = 200 per Quadrat-Millimeter Querschnitt anzunehmen."

5. „Man kann den Elasticitätscoëfficienten nicht nur durch die Verlängerungen, sondern auch durch Biegung und Drehung bestimmen, aber man gewinnt durch diese verschiedenen Methoden auch verschiedene Werthe und um sie auf eine gleiche Ziffer zurückzuführen, wird man in jedem Fall einen constanten Coëfficienten, der von der Operation selbst abhängt, zu ermitteln haben."

6. „Die leichteste Methode um den Elasticitätscoëfficienten zu erhalten, ist, den Körper an beiden Enden zu unterstiutzen und ihn in der Mitte zu belasten."

Die Beobachtungen Paccinotti und Peri's erscheinen so exact, als sie es ohne Anwendung des Kathetometers sein konnten; auch das Gesetz der Formveränderungen, welches in dem ersten der obigen Grundsätze enthalten ist, stimmt mit jenem, der bei den Metallen statt hat, vollkommen überein. Indessen bleibt einige Ungewissheit bezüglich der aus den Versuchen abgeleiteten Coëfficienten und beziiglich des Vergleichs der Methoden untereinander; denn diese Auioren haben vernachlässigt, den Theil des Baumes, woher die Stäbe genommen sind, sowie den Grad der Feuchtigkeit derselben bei den verschiedenen Versuchen in Rechnung zu ziehen. Im Verfolg dieser Árbeit wird man sehen, dass 
die Elasticität nicht in allen Theilen eines Baumes dieselbe ist, dass sie bemerkenswerth mit dem Feuchtigkeits-Grade wechselt, und dass dieser letztere selbst in so kleinen Stäben, wie sich deren die Autoren bedient haben, schr variabel ist. Die Ergebnisse der Paccinotti'- und Peri'schen Beobachtungen, welche unter abweichenden. Umständen an dem nämlichen Holze und jene, welche an diversen Holzarten gewonnen wurden, sind demnach denn doch nicht ganz vergleichbar untereinander.

Wir bemerken ferner überdiess, dass nach den bekannten Formeln, welche den Elasticitätscoëfficienten mit der Schallsgeschwindigkeit in Verbindung bringen, der Coëfficient $\mathrm{E}^{\prime}$, den Paccinotti und Peri eingeführt haben, eine Proportionalgrösse zum Quadrat der Schallgeschwindigkeit ist, woraus, wenn $\mathrm{E}^{\prime}$ constant wäre, folgt, dass auch die Schallgeschwindigkeit für alle Holzarten dieselbe scin miisste. Nun wird sich aber bald zeigen, dass diese nicht blos bei dem verschiedenen Stoff, sondern sogar in den verschiedenen Theilen eines und desselben Baumes und in ein und demselben Stabe, je nach seinem Trockenheitsgrade, variirt. Es versteht sich endlich, dass, weil der Elasticitätscoëfficient $\mathrm{E}$ im allgemeinen mit der Trockene zunimmt, während das specifische Gewicht $\mathrm{G}$ sich vermindert, $\mathrm{E}^{\prime}=\frac{\mathrm{E}}{\mathrm{G}}$ in diesem Falle um so mehr zunehmen werde.

Wir werden diesen historischen Abriss über die bis heute durchgeführten Untersuchungen der mechanischen Eigenschaften des Holzes durch ein Resumé der Ansichten der Autoren über jene Fragen beschliessen, die uns als die wesentlichsten erschienen sind, und welche so zu sagen den Cadre für unsere eigenen Versuche gebildet haben.

Hier folgen nun diese Fragen in ihrer natiirlichsten Aufeinanderfolge:

I. Welche Wirkung übt auf die Hölzer eine allmählig wachsende Belastung aus? Nach welchen Gesetzen finden dic daraus hervorgehenden Formveränderungen statt, und welches sind die auf die Hölzer behufs Bestimmung ihrer mechanischen Eigenschaften anwendbaren Methoden?

Charles Dupin hat die Proportionalität zwischen den Belastungen und den hervorgebrachten Wirkungen für den Fall nachgewiesen, dass erstere sehr klein sind.

Paceinotti und Peri haben gezeigt, dass dieses Gesetz fïr alle Belastungen, selbst für jene, whlehe dem Bruch nahe 


\section{$-11-$}

kommen, giltig ist, vorausgesetzt dass man den permanenten Formveränderungen Rechnung trage. Sie haben weiters gefunden, dass man gewisse Correctionscoëfficienten anwenden miisse, um die Ergebnisse untereinander in Einklang zu bringen.

II. Variiren die Eigenschaften in ein und demselben Baume nach der Orientation, d. h. je nachdem ein Theil desselben der einen oder anderen Weltgegend zugekehrt ist?

Musschenbroeck allein hat diese Frage, und zwar nur mit Ruicksicht auf die Festigkeit behandelt; - er fand, dass sie die höchste gegen Osten ist und gerade im Süden und Westen gegen Norden passirend kleiner wird.

III. Variiren die mechanischen Eigenschaften mit dem Feuchtigkeitszustande der Hölzer?

Nach Musschenbroeck und Buffon ist die Festigkeit in den grünen Hölzern stärker, als in den trockenen. Duhamel dagegen glaubt, dass die Saftextraction ihre Festigkeit nicht geringer mache.

Endlich Hagen hat gefunden, dass sie eine Vergrösserung des Elasticitätscoëfficienten herbeiführe.

IV. Variiren die Eigenschaften in ein und demselben Baume, in ein und derselben Höhe, in der Richtung vom Mittelpunkt gegen den Umfang, und rach welchem Gesetze?

Was die Dichte anbelangt, hat $\mathrm{Buffon}$ gefunden, dass sie in jungen Bäumen nach einer arithmetischen Progression vom Centrum gegen den Umfang hin abnimmt und dass zur Zeit der Reife die Dichte überall so ziemlich dieselbe sei. Buffon hat jedoch nur Eichen untersucht.

Nach Duhamel findet sich die grösste Dichte bei kräftigen Bäumen im Kernholz, während bei in der Ruickbildung begriffenen Bäumen das Kernholz oft leichter ist, als jenes zwischen Kern und Umfang.

Bezüglich der Festigkeit findet Musschenbroeck, dass sie im Kern des Baumes am schwächsten, nahe am Splint grösser, als nahe am Kern ist, und dass sie ihr Maximum in ciner mittleren Region erreiche, was mit der über die starken Fichten des Nordens ausgesprochenen Ansicht Duhamel's iibereinstimmt.

Buffon hat im Gegentheil gefunden, dass die Festigkeit grösser im Centrum, als am Unfang sei, aber dass die Regel für Bäume von 100 bis 110 Jahren aufhört, wahr zu sein. 
Betreffs der Elasticität hat Hagen keine sehr grosse Differenz zwischen Kern und Splint gefunden.

V. Variiren diese Eigenschaften in ein und demselben Baume mit der Höhe:

1. Im Sinne der Fasern für jede Jahresholzschicht oder für den ganzen Baumstamm?

2. Im Sinne des Halbmessers senkrecht zur Faser?

3. Im Sinne der Tangente zu dem Jahrringe senkrecht zur Faser?

Nach Buffon und Duhamel nimmt die Dichte des Fiolzes vom Fusse gegen die Krone des Baumes hin ab.

Musschenbroeck nimmt keinen merklichen Unterschied für die Festigkeit des Holzes in den Zweigen und im Stamm, zu was immer für einer Höhe, an. Nach Buffon wäre dagegen die Festigkeit im Fusse des Baumes grösser als am Gipfel.

Diese Ergebnisse beziehen sich nur auf die Festigkeit im Sinne der Fasern.

VI. Welche Beziehung besteht zwischen den mechanischen Eigenschaften im Sinne der Fasern und den zwei auf diese senkrechten Richtungen zu verschiedenen Höhen im Baume?

Wir haben in dieser Richtung nur einige von Savart, Wheatstone und Hagen angestellten Versuche vorgefunden, welche die Beziehungen unter den Elasticitäten in ein und derselben Höhe des Baumes untersucht haben.

VII. Was für einen Einfluss hat das Alter der Bäume?

Wir haben schon die Ansichten der Autoren bezüglich der Veränderungen angeführt, welche mit dem Alter in den verschiedenen Jahrringen auftreten. Was nun das Holz, in seiner Gänze aufgefasst, betrifft, so sagt Buffon, dass es mit dem Alter an Kraft zunehme, aber diese Frage ist noch nicht genügend erforscht worden. Dasselbe gilt für die folgenden Fragen.

VIII. Welchen Einfluss hat die Breite der Jahrringe?

Nach Buffon ist das Holz, welches auf demselben Boden am schnellsten erwächst, das festeste, und jenes, welches langsam wachst und daher sehr schmale Jahrringe hat, ist das schwächste.

Duhamel scheint einer entgegengesetzten Ansicht zu sein. Er behauptet, dass die Fichten des Nordens, welche von ausgezeichneter Qualität sind, sehr dünne Jahresschichten haben, was auch aus den interessanten Untersuchungen von Bravais und Martins (Recherches sur la croissance du Pin silvestre, Memoiren der Brüsseler Akademie) hervorgeht. 


\section{$-13-$}

IX. Welchen Einfluss hat die Exposition?

X. Welchen Einfluss hat die Bodenbeschaffenheit?

Nach Musschenbroeck erzeugen die sandigen Böden minder kriaftiges Holz, als thoniger Grund; und nach D uhamel sind jene Terrains, welche geeignet sind schöne Bäume hervorzubringen, nicht diejenigen, welche das $\mathrm{Holz}$ bester Qualität ergeben.

XI. Welche Beziehungen bestehen unter den verschiedenen mechanischen Eigenschaften des Holzes?

Buffon hat beobachtet, dass die Festigkeit der Dichte proportional sei; Charles Dupin, dass die Dichten gleichzeitig, aber nach einem geringeren Maassstab, mit den Widerständen gegen die Biegung wachsen. Der letztere Autor hat auch bewiesen, dass es keine constante Beziehung zwischen Festigkeit und Elasticität der Hölzer gebe.

XII. Welche Mittelzahlen kann man einführen, und welche sind die Konsequenzen, die man aus denselben für die Praxis ziehen kann?

Man sieht aus der Tabelle Nr. I., dass die bisher angestellten Versuche zu Ergebuissen geführt haben, welche so sehr divergiren, dass man folgende Grenzen aufstellen muss:

Dichte Elasticitätscoëfficient Festigkeit

\begin{tabular}{|c|c|c|c|c|}
\hline che & & $0 \cdot 616$ & $500-1600$ & $5-12$ \\
\hline Buche & & $0.600-0.811$ & $950-1483$ & $8-12$ \\
\hline Tanne & & $0.443-0.703$ & $611-1615$ & $5-9$ \\
\hline Fichte & & $0.396-0.753$ & $433-1776$ & $4-8$ \\
\hline
\end{tabular}

Selbst die Genauigkeit der angewendeten Methoden angenommen, lassen sich diese grossen Divergenzen aus den Bedingungen, unter denen diese Beobachtungen angestellt wurden, erklären.

Was nun immer der Grund sei, die Ergebnisse erweisen die Nothwendigkeit einer neuen Studie über die mechanischen Eigenschaften des Holzes, unter Berücksichtigung aller Umstände die sie modificiren können und zur Vervollständigung der theoretischen Untersuchungen durch Versuche, welche im Hinblick auf die Praxis eingeleitet werden. 


\section{ZWEITER THEIL.}

\section{Beschreibung der Versuche und der zu den- selben dienenden Vorrichtungen.}

In der vorliegenden Arbeit wurden vorerst die Verschiedenheiten aufgesucht, welche bei den gleichen Hölzern in Folge des Feuchtigkeitsgrades und in den verschiedenen Theilen desselben Baumes auftreten, ferner die mechanischen Eigenschaften der Bäume mit Rücksichtnahme der auf sie einflussnehmenden Umstände, endlich die mechanische Beschaffenheit des Eichen- und Tannenholzes in den in der Praxis üblichen Formen und $\mathrm{Ab}$ messungen.

Wir werden einzeln die Methoden und Vorrichtungen beschreiben, welche wir bei diesen verschiedenen Untersuchungen angewendet haben.

Wir haben Bäume verwendet, welche in jenen Lokalitäten der westlichen Abhänge der Vogesen erwachsen sind, in denen die forstlichen Arbeiten von einem von uns besorgt wurden. Die Hölzer wurden aus einem beiläufig 4000 Hektaren umfassenden Waldgebiet ausgewählt. Die dort herrschenden Verhältnisse, welche indessen genügend viel Varianten darbieten, sind genau bekannt, was unmöglich der Fall ist, wenn man Hölzer vornimmt, die uns der Handel zufällig zur Verfügung stellt.

Wir haben auf jenem Terrain alle Details der Arbeit verfolgt, so dass wir der Auswahl und Vorbereitung der Bäume dieselbe Sorgfalt zuwenden konnten, wie den Versuchen selbst.

Die hauptsächlichsten Umstände, denen wir bei Auswahl der Bïume Rechnung tragen zu müssen glaubten, sind die geo- 
logische Beschaffenheit des Bodens, die Guite des Grundes, die Exposition, das Alter, der Zustand des Waldes, und ausserdem haben wir bei einigen den Einfluss der Fällung während der Saftzeit untersucht. Die gefällten und zu den Versuchen bestimmten Bäume wurden nach der geologischen Nutur des Bodens classificirt (siche Tabelle II). Jeder erhielt bei der Faillung eine OrdnungsNummer, und an der Seite eine Marke, welche gestattete später dic ursprüngliche Position des Baumes zu den Weltgegenden wieder aufzufinden.

Die Forste sind auf drei verschiedenen Bodenarten erwachsen: Vogesensandstein, bunter Sandstein und Muschelkalk. In jeder derselben konnten wir die drei folgenden Gütegrade der Erde unterscheiden: trocken und dürr, fruchtbar, nass.

Der Vogesensandstein allein hat uns genügend viele differirende Expositionen dargeboten, $u$ m denselben Rechnung tragen zu können, die beiden anderen Gebiete hingegen bilden nur Plateaux.

Um den Einfluss der Exposition auf das Holz zu finden, wurden in jeder eigenthiumlichen Lage folgende Bäume gefällt: Vom Vogesensandstein je eine Eiche (Quercus sessiliflora),

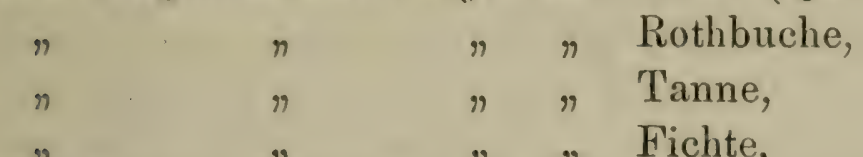

vom bunten Sandstein je eine Eiche (Quercus pedunculata),

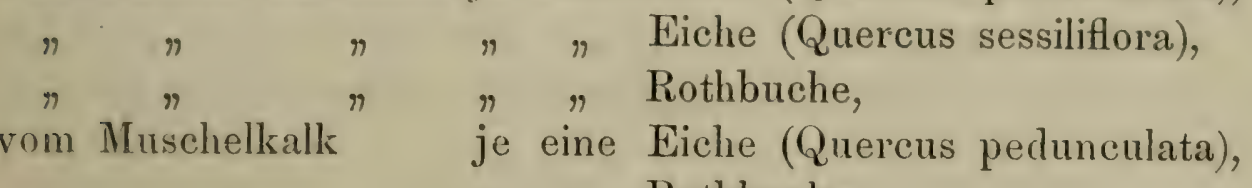

" " " Rothbuche.

Um den Einfluss des Alters zu erkennen, haben wir von einer gewissen Localität im Vogesensandstein, Serien von Eichen, Buchen und 'T'annen von verschiedenem Alter und verschiedener Stärke entnommen.

Wir haben ferner einige andere Species von Hölzern, die in diesen Forsten erwachsen, füllen lassen. Endlich wurden, da alle obigen Bäume ausser der Saftzeit gewonnen worden sind, mehrere Exemplare wïhrend des Hauptsafttriebes gefïllt.

Die Eichen und Tannen, welche uns zu den Experimenten mit Stücken von handelsüblicher Form und Grösse gedient haben (siche Tabelle III.), waren getrocknet worden, erstere mehrere Jahre hindurch, letztere in der Dauer eines Jahres.

Wir haben in der eben erwähnten Tabelle den Ursprung und die verschiedenen Bedingungen, unter denen sie erwachsen sind, angegeben. 


\section{$-16-$}

Die Gesammtanzahl der für unsere Zwecke gefällten Bäume betrug 94 , nämlich:

\begin{tabular}{|c|c|}
\hline Iichen & 31 \\
\hline Buchen . & 16 \\
\hline Tannen. & 28 \\
\hline Fichten . & \\
\hline Weissbuchen & \\
\hline Birken & \\
\hline Akazie & \\
\hline
\end{tabular}

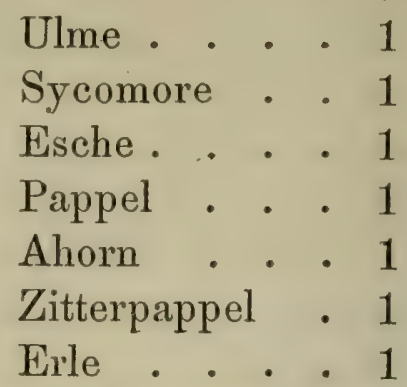

Die sämmtlichen Bäume, mit Ausnahme der zu handelsüblichen Formen verarbeiteten und der zur Saftzeit gefällten, wurden unmittelbar nach dem Hiebe in einen Hangar gebracht und dort geschützt vor den Einflüssen der Atmosphäre so viel als möglich in dem Zustande im Momente der Fällung zu erhalten.

\section{A) Untersuchnng der mechanischen Beschaffenheit des Holzes eines und desselben Baumes.}

Diese Untersuchung hat die Aufgabe die unter I. bis VI. gestellten Fragen zu beantworten. Es erhellt aus der Fassung jener Fragen, dass es nothwendig war, nicht blos verschiedenen Richtungen im Innern des Baumes zu folgen, sondern dass man auch in verschiedenen Höhen und in verschiedenen Jahrringen mit Rücksicht auf ihre Lage gegen die vier Hauptpunkte der Windrose Studien über die mechanischen Eigenschaften machen musste.

Für diese Versuche haben wir 2 Eichen, 2 Buchen, 2 Tannen und je einen Baum der anderen Arten ausgewählt. Dieselben wurden in Trumme (Cylinder) von 2 Meter Länge geschnitten, von denen jedes eine Nummer, unabhängig von der OrdnungsNummer des ganzen Baumes, erhielt. Man gieng dabei vom Fusse aus und konnte aus dieser neuen Nummer auf die Höhe des Trummes im Baume schliessen. Auf der Basis jedes Trumms haben wir eine 2 Centimeter dicke Platte (Scheibe) senkrecht zur Axe weggenommen und gleichfalls numerirt.

Die Walzen wurden nun mittelst der Säge so zerschnitten (Tafel I. Fig. 1.), dass drei Bretter entstanden, von denen das eine den Mittelpunkt enthielt und in der Richtung von Norden nach Sïden herausgenommen war, die anderen zwei senkrecht auf diese Richtung, also von Osten nach Westen liefen. Wir bezeichneten die Partien des Baumes, welche ursprünglich nach den vier Weltgegenden hin gerichtet waren. 

Fig. 1.
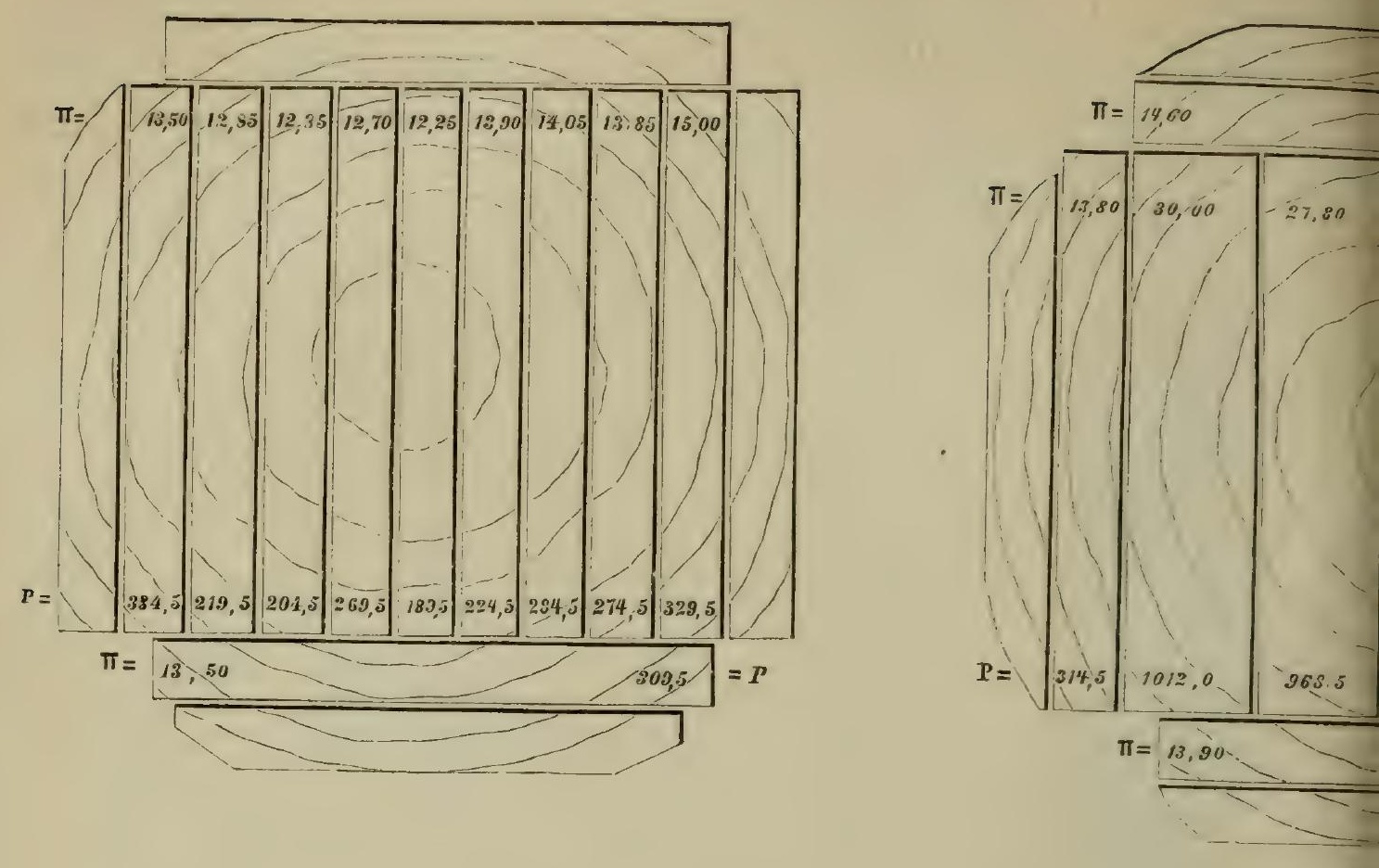

Fig. 4.
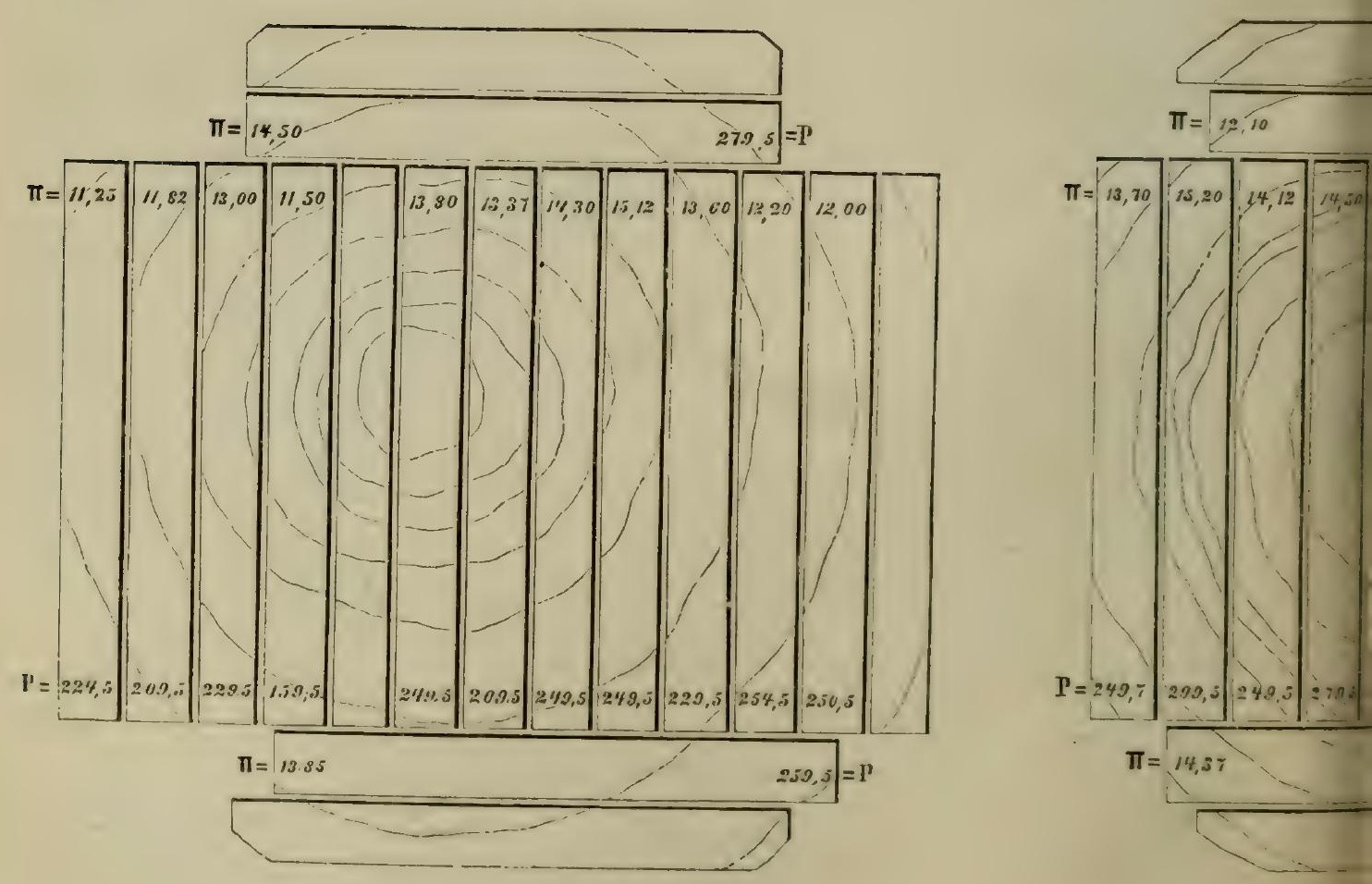
Fig. 3.
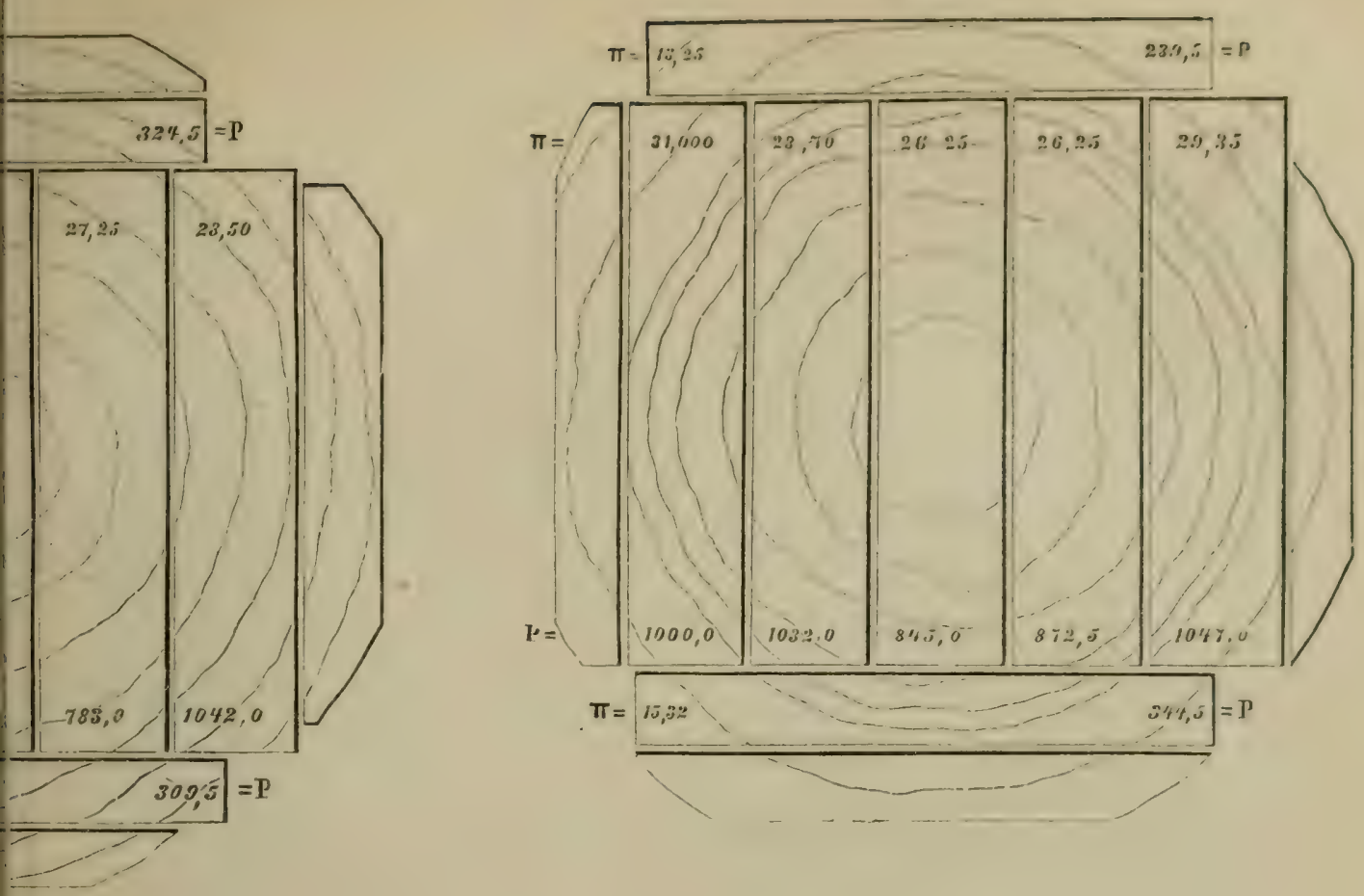

5.

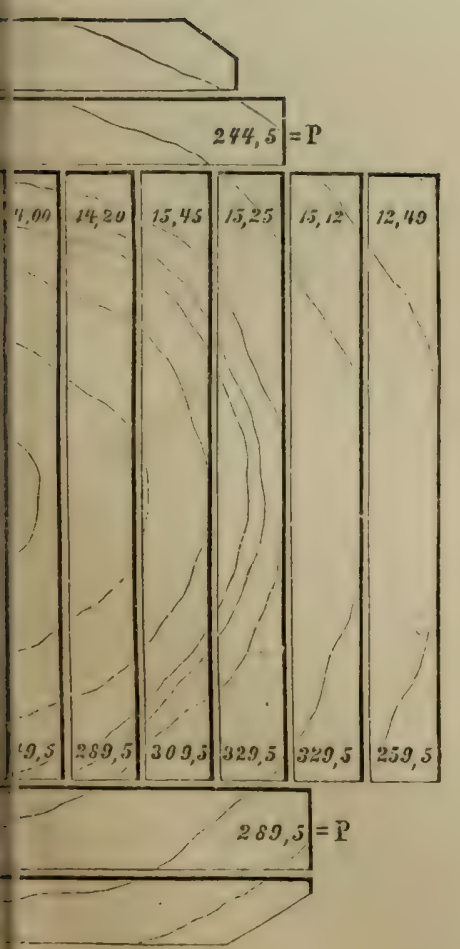

Fig. 6.

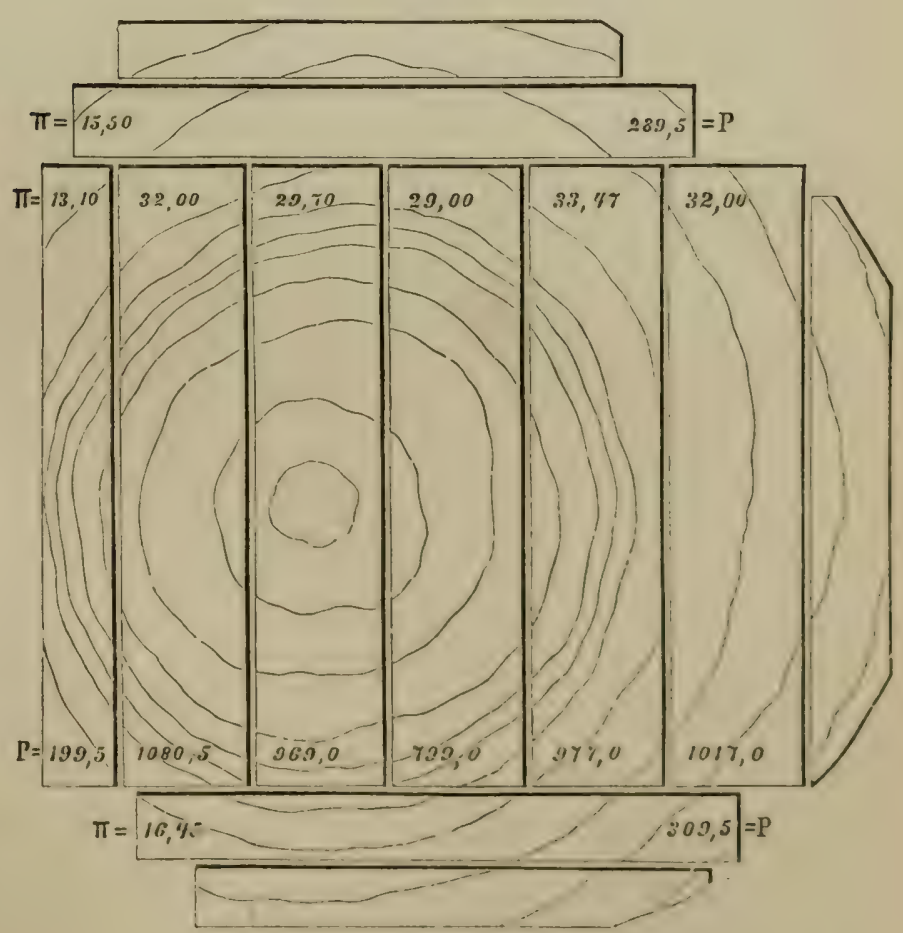



Nachdem nun die Jahrringe, die in den Brettern enthaltent waren, sorgfältig gezihlt worden sind, wurden diese Bretter ihrer ganzen Länge nach, abermals der Faser folgend, in der Art zerschnitten, dass wir Serien von prismatischen Stäben gewannen. Ein Parellelopiped enthiclt das Centrum, das Herz des Baumes, die anderen von der Mitte gegen den Umfang hin den Latten entnommenen Stangen enthielten je vier immer dieselben Jahrringe.

Wenn wir mehrere Trumme desselben Baumes einer Prïfung unterwarfen, so waren wir darauf bedacht, in jedem Trumme die Stäbe aus denselben Jahrringen zu nehmen, so dass die Prismen sich gegenseitig fortsetzten.

Diese Stäbe hatten beiläufig dieselbe Länge, wie die Trumme, nämlich nahezu zwei Meter. Sie wurden vollkommen genau mit dem Hobel zugerichtet und auf gleiche Dimensionen georacht. - Jeder von ihnen erhielt drei Nummern; die erste den Baum bezeichnend, dem er angehörte, die zweite das Trumm, aus dem er genommen wurde und die dritte die Entfernung vom Centrum angebend. Das die Axe enthaltende Prisma erhielt immer die Nummer 1. Ueberdiess wurde jede Stange mit einem Buchstaben markirt, der die Orientation nach der Weltgegend angab.

Von den Scheiben, die oben erwähnt wurden, liessen wir zwei Barren herausarbeiten, den einen in der Richtung des Radius vom Mittelpunkt bis zum Umfang, den andern im Sinne der Tangente an die Jahrringe. Diese Barren trugen die Nummern des Baumes, des Trummes und die Buchstaben R oder T - Radius oder Tangente bedeutend. - Nach den Versuchen Savart's konnten wir das erste der beiden Stiicke einem beliebigen Radius folgend nehmen. Das zweite wurde so nahe als möglich am Umfange und zwar an jener Stelle herausgeschnitten, an welcher der Krümmungshalbmesser am grössten, also die Krümmung am kleinsten war.

Nachdem eine Partie Stäbe zum Behufe der Bestimmung der Festigkeit im grünen Holze zerbrochen werden musste, waren wir genöthigt von diesen Dupplikate vorzurichten; wir suchten das dadurch nahezu zu erreichen, dass wir immer der Dicke der Bretter nach zwei nnmittelbar benachbarte Stäbe aus denselben Jahrringen anfertigten, welche dann auch als identisch angesehen werden konnten. Die eine dieser Serien von Stiiben wurde ummittelbar allen Versuchen unterzogen, ein Theil derselben diente jedoch blos jenen für die Festigkeit. Die Staibe, welche ganz erhalten wurden, kamen in einem Ofen zur Trocknumg. Die zweite Seriu 
wurde erst nachdem sie einige Zeit im Versuchsraum aufbewahrt gewesen, zur Untersuchung gebracht. Man trachtete soviel als möglich die Stäbe ganz zu erhalten, trocknete sie in der freien Luft und an der Sonne, um sie dann neuerdings zu prüfen.

Wir haben demnach die mechanische Beschaffenheit von Längsstäben studirt in vier verschiedenen Feuchtigkeitszuständen.

1. Grünes Holz. Als solches bezeichnen wir jene Stäbe, die wir unmittelbar nach der Gewinnung der Arbeit unterzogen haben.

2. Einige Zeit im Versuchslocale aufbewahrtes Holz. Diese Stäbe befanden sich einige Zeit hindurch im Laboratorium, d. h. in einem geschlossenen Locale unter sich gleich bleibenden Umständen.

3. Luftrockenes Holz. Die Prismen wurden unter einem mit Fenstern versehenen Dache, von der Einrichtung, dass man sie bei Regen und während der Nacht schliessen konnte, mindestens einen Monat hindurch, untergebracht, wo sie der directen Einwirkung der Sonne und des Luftzuges ausgesetzt waren.

4. Künstlich getrocknetes Holz. Diese Stäbe hielten sich circa 14 Tage in einem geschlossenen Trockenkasten auf, in dem eine Temperatur von 40-50 Grad Celsius herrschte.

Da wir unter analogen Verhältnissen durch directe Versuche iiber die Trocknung von Brennholz bei sehr trockenem Holze die Erfahrung machten, dass alles derselben Höhe des Stammes angehörige Holz in gleichem hygrometrischen Zustand war, so haben wir angenommen, dass in Folge der gehabten Vorsicht in jedem Falle alle Stäbe, die demselben Trumme entstammten, sich im gleichen Feuchtigkeitszustande befanden. Darauf gestützt, bestimmten wir den Wassergehalt der Stäbe in folgender Weise:

Wir haben durch doppelte Wägungen das absolute Gewicht der Stäbe in diesen verschiedenen Stadien der Trocknung ermittelt, aus welchen sich die successiven Wasserverluste für jeden ergaben. Diese stellten sich für sämmtliche Stäbe eines Trummes als auffallend gleich gross heraus. Um endlich den absoluten Wassergehalt der am meisten getrockneten Stäbe zu eruiren, das sind die im Ofen gedörrten, haben wir dieselben senkrecht zur Länge zersägt und von jedem eine gleiche Quantität Sägespäne gesammelt. Alle von demselben Trumm herrührenden Späne wurden in einem sehr dünnen Rohre untergebracht und dieses mehrere Tage lang unter cinem trockenen Recipienten der Luftpumpe gesetzt, nachdem es schon vorher wiederholt bis auf $140^{\prime \prime}$ erhitzt worden war. Dieses Verfahren wurde so lange wiederholt, bis 
die Späne keinen messbaren Verlust an Gewicht mehr zeigten. Der totale Verlust ergab uns die im gedörrten (im Ofen getrockneten) Holze enthaltene Wassermenge. Indem man diesem Wasserquantum die nach jedem Trocknungsstadium entstandenen Gewichtsdifferenzen beifügte, erhielt man die Wassergehalte der Hölzer in den verschiedenen Feuchtigkeitszuständen. Alle Resultate dieser Kathegorie von Versuchen sind in der Tabelle V vereinigt.

Wir müssen noch bemerken, dass die zwei senkrecht zur Faserrichtung im Sinne des Radius und der Tangente herausgearbeiteten Barren in einem einzigen Zustande geprüft wurden, und zwar in dem der spontanen Trocknung im Laboratorium.

Bei jedem der vier Feuchtigkeitszustände hatten wir folgende Eigenschaften des Holzes zu untersuchen: das absolute Gewicht, die Dichte, die Schall-Leitungsfähigkeit (Geschwindigkeit), das Elasticitätsgesetz, die Elasticitätsgrenze und ihren Coëfficienten, die Maximal-Ausdehnung und die Festigkeit. Die Versuche wurden in der folgenden Ordnung und ohne Unterbrechung vorgenommen, um so viel als möglich eine Veränderung im Trockenheitsgrade der Stäbe zu vermeiden.

1. Dichte. Wir haben uns dreier verschiedenen Methoden, die uns am leichtesten ans Ziel führen konnten, bedient. In manchen Fällen berichtigte die eine Methode die durch die anderen gewonnenen Resultate.

Einige Bestimmungen haben wir mit Hülfe des Volumometers von Regnault gemacht; da aber unser Kathetometer bei anderen Versuchen in Anwendung war und bei jenen nicht entbehrt werden konnte, wurden auf diese Art nur wenige Versuche durchgeführt.

Am häufigsten haben wir ein verkorktes Fläschchen, von 5 Centimeter Höhe und 2 Centimeter Durchmesser, das mit Quecksilber gefüllt war, angewendet. In dieses Fläschchen haben wir zwei Stückchen von gleicher Länge, von den beiden Enden jedes Stabes, versenkt. Nachdem die adhärirenden Luftblasen so viel als möglich weggeschafft worden waren, wurde das verdrängte Quecksilber gewogen. Aus diesem Gewichte und jenem der Stabstückchen konnte man die specifischen Gewichte rechnen.

Wenn wir zur Annahme veranlasst waren, dass die Dichte nicht in der ganzen Lünge des Stabes die gleiche sei, was namentlich bei den senkrecht zur Faser genommenen Barren der Fall ist, so haben wir die Dichte aus dem absoluten Gewicht des gauzen Stabes oder Barrens und dessen Volumen abgeleitet. Das 


\section{- $20-$}

letztere ist bestimmt worden, indem man die Länge mit einem Metermassstab, die Breite und Dicke mit einem Sphärometer gemsssen hatte. Die Abmessungen zeigten wenig Abweichungen an demselben Stabe, dank der Sorgfalt, welche man auf die Anfertigung derselben verwendet hatte. Die Mittelzahlen der Abmessungen sind in Tabelle $\mathrm{Nr}$. XV eingetragen.

2. Schallgeschwindigkeit. Wir haben diese für die Stäbe nach der Chladni'schen Methode mittels longitudinaler Schwingungen bestimmt, nachdem man die Stablänge genau gemessen hatte. Einer von uns setzte ihn in Vibration, indem er ihn in der Mitte haltend an einem seiner Enden rieb, während der andere denselben Schall auf einem Differential-Tonmesser hervorrief, der genau mit einer Normal-Stimmgabel (Diapason normal) übereinstimmt. Die Länge der Saite, welche diesen Ton lieferte, auf diese Art gefunden, ergab uns durch Rechnung die entsprechende Zahl von Schwingungen und daraus die Geschwindigkeit des Schalles im Stabe.

Was die Transversalbarren anbelangt, so gestatteten sie wegen der geringen Länge die Hervorrufung von Longitudinalschwingungen nicht und wir konnten desshalb die Schallgeschwindigkeit nur durch den Elasticitätscoëfficienten (aus Transversalschwingungen abgeleitet) finden.

3. Elasticitätsgesetz. Wir haben diese Gesetze durch Ausdehnungen zu suchen unternommen, indem wir die Stäbe bei drei verschiedenen*) Trockenheitsgraden einer stufenweis zunehmenden Belastung unterworfen haben. Sowohl die permanenten als die elastischen Ausdehnungen oder besser Verlängerungen wurden mit dem Kathetometer gemessen. Wir haben uns einer Vorrichtung bedient, welche früher von einem von uns bei Versuchen mit Metallen und Legirungen verwendet wurde. Unsere Versuche wurden bis zum Bruch geführt und haben wir hiebei denselben Vorgang und dieselben Vorsichten beobachtet, wie bei jenen Versuchen. Es wäre viel zu umständlich, die Details aller Versuchsreihen ïber Verlängerung der Stäbe anzugeben, welche wir zu machen hatten. Wir beschränken uns denn darauf, blos ïber einige derselben in Tabelle IV, als Beispiele dafür wie wir vorgegangen sind, $\mathrm{zu}$ berichten.

Wir haben auf diese Art unter Einem die mittlere elastische Ausdehnung für eine bestimmte Belastung, die aufeinanderfolgen-

*) Die kïnstlich getrockneten Stïbe sind zn gebrechlich, um einer Verlingerung unterzogen werden zu kimmen, namentlich jene aus Akazienholz dulden keine derartige Inanspruchnahme. 
den permanenten Verlängerungen und endlich die Maximalausdehnung bestimmt. Die erste dieser Daten dient zur Berechnung des Elasticitiatscöfficienten, die zweite zur Auffindung der Elasticitätsgrenze.

Die senkrecht zur Faser herausgeschnittenen Barren sind zu kurz, um Ausdehnungen unterworfen werden zu können. Wir haben deshalb zur Methode der transversalen Schwingungen unsere Zuflucht genommen. Gewöhnlich haben wir uns des tiefsten Tones bedient, welchen die freien Enden geben, wenn man den Holzstab an dem Orte eines der Knotenpunkte hält, die bei dieser Art von Vibration sich bilden.

Man ruft den Ton durch Streichen des näheren Endes mit einem starken Geigenbogen hervor. Jeder dieser Knotenpunkte ist am Ende des ersten Viertels der Totallänge von den Enden res Barrens situirt. Wenn der so hervorgerufene Ton zu tief war, $u m$ ihn auf dem Sonometer leicht zu erhalten, so haben wir Vibrationen erzeugt, die einen höheren Ton mit drei, vier oder selbst fünf Knoten ergaben. Wenn man die Zahl der Schwingungen des tiefsten Tones mit $3^{2}$ ausdrückt', so sind die correspondirenden Schwingungszahlen der anderen Töne sehr nahe, die Quadrate der ungeraden Zahlen 5, 7 und 9. Mit Hülfe der bekannten Formeln konnten wir nun den Elasticitätscoëfficienten ableiten. In jedem Falle haben wir die Zahl und Lage der Knotenpunkte mittelst Sand, den wir auf die obere Fläche des Barrens gestreut haben, sichergestellt.

Wir müssen bemerken, dass nur homogene Substanzen immer denselben Ton geben, ob man die transversalen Schwingungen im Sinne der Breite, oder im Sinne der Dicke an einem parallelopipidischen Stabe hervorruft, was demnach beim Holze nicht der Fall ist. Für dieses Materiale gewinnt man im allgemeinen einen etwas grösseren Elasticitäts-coëfficienten, wenn der Stab die Schwingungen im Sinne der Fasern ausführt, als wenn sie senkrecht auf diese stattfinden. Immerhin war bei der Mehrzahl der Versuche die Differenz eine sehr geringe, und wir konnten in jedem Falle das Mittel aus den erhaltenen Ziffern als wirklichen Coëfficienten annehmen. Alle Daten dieser Untersuchungen sind in Tabelle XV enthalten.

4. Festigkeit. Diese Eigenschaft ist durch Zerbrechen der Stäbe und Barren im Wege der Spannung durch Zug ermittelt worden. Es wurde Sorge getragen, denselben eine rollkommene senkrechte Stellung zu geben und das Auflegen der Gewichte nur allmählig und ohne Stösse zu bewerkstelligen. 


\section{B) Untersuchung der mechanischen Beschaffenheit des Holzes verschiedener Bäume.}

Diese Untersuchung, welche die unter Nr. V, VII, VIII, IX, X aufgestellten Fragen in sich begreift, konnte man entweder mit den ganzen Stämmen oder blos mit Theilen derselben vornehmen.

In dem ersten Falle wäre es unmöglich gewesen, den Einfluss der Höhe der Bäume zu studiren, ausserdem hätte die fortwährende Abänderung der Vorrichtungen, je nach der verschiedenen Länge der Stämme grosse Schwierigkeiten dargeboten, so wie es auch schwer durchführbar gewesen wäre, für die, an unregelmässig konischen Körpern, wie es die ganzen Bäume sind, erhaltenen Versuchsergebnisse die gewöhnlichen Formeln zu verwenden. Wenn man dieselben andererseits hätte auf eine regelmässige Form bringen wollen, so hätte man sie unter Verlust eines grossen Theils ihrer äusseren Schichten nahezu unter jene Bedingungen gebracht, wie sie sich bei den im praktischen Leben verwendeten Stämmen vorfinden, mit welchen wir uns in der dritten Abtheilung dieser Arbeit beschäftiget haben.

Wir haben daher vorgezogen bei der Vergleichung verschiedener Bäume, Trumme von 2 Meter Länge, die in der gesundesten und regelmässigsten Partie eines jeden Stammes gewonnen wurden, zu verwenden. Was den Einfluss der Höhe betrifft, so untersuchten wir sie an den Trummen, welche von ein und demselben Baume kamen und von denen abwechselnd die einen ganz belassen und die anderen in Stäbe zerlegt wurden, so dass z. B. bei einem in 6 Stücke getheilten Baume, die Trumme Nr. 1, 3 und 5 (von der Basis aus gezählt) nach den bestimmte Jahrringe enthaltenden Stäben, die Trumme 2, 4 und 6 aber im ganzen untersucht wurden.

Bei diesen Untersuchungen haben wir blos die Dichte und den Elasticitätscoëfficienten bestimmt. In der That würde es auch mit Trummen von solcher Länge und zuweilen von sehr grossem Durchmesser schwierig gewesen sein, bis zum Bruch zu gehen. Uebrigens hängen die Werthe für die Elasticitätsgrenze und für die Festigkeit zu sehr von der Ausführung des Versuches ab, and sind, wie es in der Natur der Sache liegt, zu ungenau, um dort in Betracht zu kommen, wo es sich nur um kleine Differenzen handelt, wie es jene sind, welche wir bei diesem Theil unserer Arbeit aufzusuchen gehabt hatten. 


\section{Tafel II.}

Fig. 1.

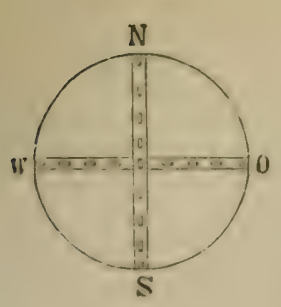

Fig. 2.

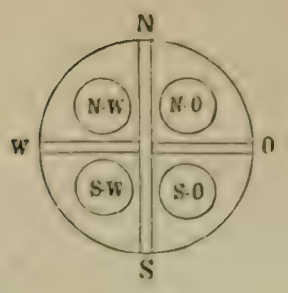

Fig. 3.

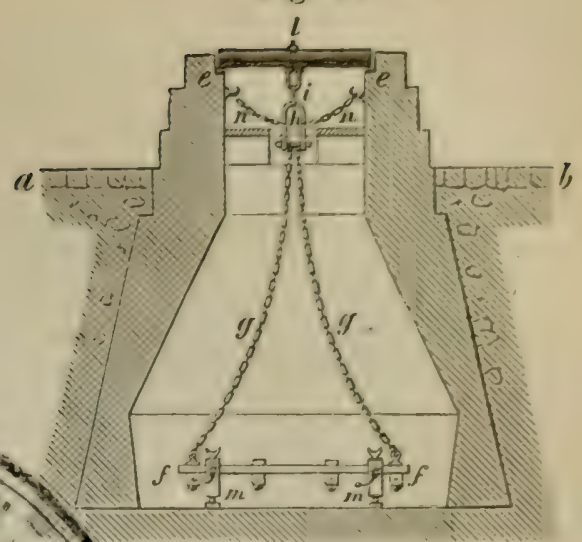

Fig. 5.

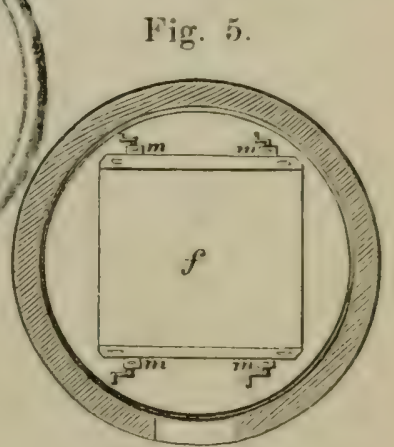

Fig. 7.

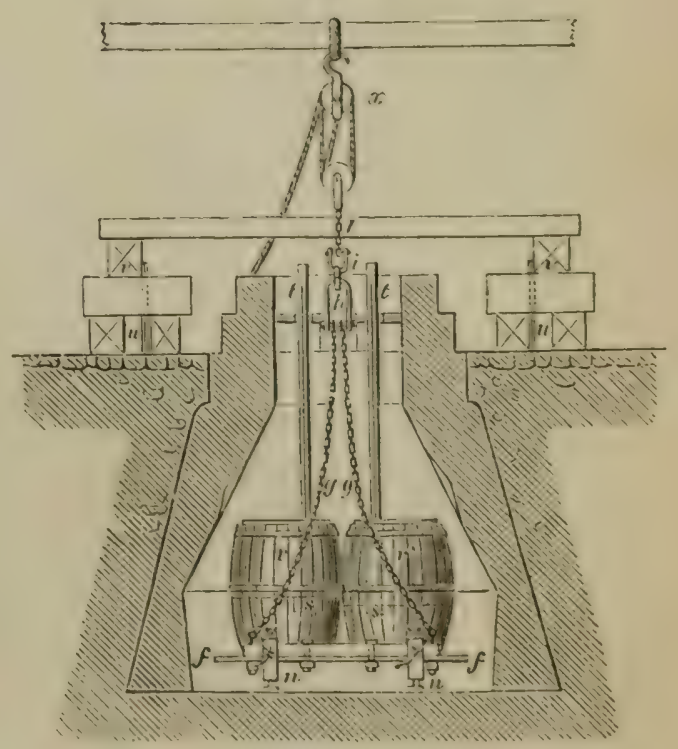

Fig. 8.

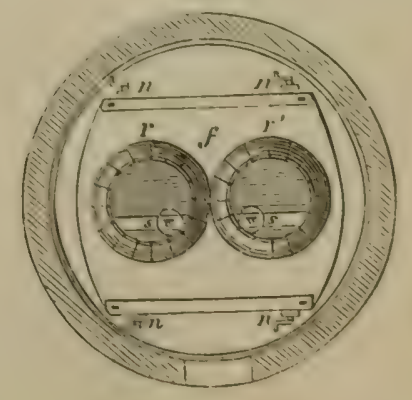

Maassstab für die Fig. 3-9. 

Die Trumme wurden entwindet und in cylinderische Form gebracht, was bei ihrer geringen Länge leicht war, indem man an der Basis den Durchmesser nur um etwas $\mathrm{zu}$ verkleinern brauchte.

Die Dichte wurde unmittelbar vor dem Versuch aus dem absoluten Gewicht, aus der Lünge und dem mittleren Durchmesser bestimmt. Um den letzteren zu finden, nahmen wir mit einem Messband an fünf verschiedenen Punkten den dreifachen Umfang von jedem Trumm, um auf diese Weise genauere Mittelwerthe zu erlangen.

Der Elasticitätscoëfficient wurde durch Ausbiegung ermittelt. Das Trumm war an beiden Enden frei unterstizt und die Last in der Mitte wirksam.

Damit dieser Vorgang bei so starken Stuicken eingeschlagen werden kann, müssen folgende Bedingungen erfüllt werden:

1. Die Auflagen müssen so stark sein, dass sie eine 10.000 Kilogrammes übersteigende Belastung ertragen.

2. Muss eine so grosse Belastung mit Leichtigkeit und ohne Strisse aufgelegt und weggenommen werden können.

3. Muss die Ausbiegung mit grosser Genauigkeit gemessen werden können.

Wir bedienten uns zum Behufe dieser Versuche einer konischen, ausgemauerten, oben offenen Grube, in welche man durch einen eigenen unterirdischen Gang leicht gelangen konnte. Aus den in Tafel I enthaltenen Figuren 3, 4, 5, 6 ist sowohl die Form der Grube als auch die des unterirdischen Aufbaues ersichtlich. Ueber dem Versuchsraum wurde ein Schoppen errichtet.

Auf der Futtermauer der Grube errichteten wir zwei aus Quadern aufgeführte Pfeiler, welche an der Innenseite ihrer Obertheile gusseiserne Lager, e, Fig. 32 und 4, trugen, die zur Unterstiitzung der beiden Enden des Trummes zu dienen bestimmt waren und einen grösseren Krümmungsradius als das Profil der Trumme besassen. Die ganze Anordnung war so getroffen, dass die Trumme an beiden Enden mindestens auf 4 Centimeter unterstiitzt waren, ohne mit den Hirnflächen die Quadermauer zu berühren. Wir haben in der Grube eine sehr starke rechteckige Platte, die zur Aufnahme der Gewichte bestimmt ist, an Ketten, die einerseits an den vier Ecken der Platte $f$, andererseits an einem Doppelbügel $h$ und $i$ befestigt sind, aufgehängt. Dieser Biigel wird wieder durch eine starke Vaucanson'sche Kette, die über die Mitte des zu untersuchenden Trumms gelegt ist, getragen, so dass an dieser letzteren das ganze aufgehängt ist. Auf. 


\section{- $24-$}

dem gepflasterten Fussboden der Grube werden 4 Hebewinden $m$ aufgestellt, um die Platte heben zu können, und zwei Sicherheitsketten n, die an den Säulen eingehängt waren, dienten dazu, um die Bügel und Ketten $g$ mit dem Plateau zu halten, wenn das Trumm ausgewechselt wurde und gleichzeitig dazu, um Unfälle bei einem unvorhergesehenen Bruch des Trummes zu verhiiteir. Endlich haben wir auf einer grossen gusseisernen Platte $o$ (Fig. 4), die von einem starken eichenen Klotz $p$ getragen wurde (dieser ruhte auf der Futtermaner der Grube), ein Kathetometer aufgestellt. Der Beobachter stand auf dem Mauerwerk hinter dem Kathetometer, etwa bei $q$ und konnte auf diese Art beim Gehen die Libelle nicht alteriren.

Die beiden Bügel und die Ketten wogen zusammen $184 \cdot 5$, die Platte $864 \cdot 0$ Kil.

Der Gang der Untersuchung war für jedes Trumm folgender:

Während das Plateau durch die Winden und die Aufhängeketten durch die Sicherheitsketten, welche entsprechend verküirzt worden waren, getragen wurden, legten wir das Trumm in der Art in die Lager, dass die Mitte desselben von beiden Unterstiitzungspunkten gleiche Entfernung hatte. Eine Marke zeigte diese Mitte an, und wir leyten die Vaucanson'sche Kette so iiber das Trumm, dass nur jenes Markzeichen für die Mitte innerhalb eines Kettenringes sichtbar blieb. Hierauf wurden die Haken eingehängt, die Sicherheitsketten verlängert und die Winden so weit herabgelassen, dass die Platte mit den Gewichten frei am Trumm hing und auf den Halbirungspunkt einwirkte. Das anzuwendende Gewicht ist durch eine vorhergehende Rechnung annähernd bestimmt worden. Das Trumm erlitt in Folge der Belastung eine Ausbiegung, welche entweder eine blos elastische oder gleichzeitig eine elastische und permanente sein konnte. Um nun von den beiden (für den letzteren Fall) diejenige Ausbiegung zu messen, um die es sich hier handelt, wurde die erste Beobachtung während der Action der Belastung und die zweite dann vorgenommen, wenn dieselbe mittelst der Winden gehoben, also unthätig gemacht worden war. Wie wir schon angedeutet haben, wurde diese Messung der Ausbiegung mit dem Kathetometer vorgenommen und auf jene Marke in der Mitte des Balkens bezogen. Um die Wirkung der Compression zu umgehen, wurde die Marke so niedrig als möglich angebracht. Die Messung ergab nach dem gesagten die elastische Ausbiegung, welche durch das Gewicht der Belastung, die Platte mit inbegriffen, hervorgerufen worden war. Die Ketten sammt den Haken blieben jederzeit 
angehïingt, und waren als constante Gewichtsgrösse ohne Einfluss auf die Ausbiegungsgrössen. In zwei Fällen, wo die Ketten allein himreichten, um eine Ausbiegung liervorzurufen, wurde indesscen die erste Messung unter alleiniger Belastung des Trummes durch die Ketten, die zweite nach Aushängung derselben vorgeriommen.

Jede Operation wurde zweimal vorgenommen, um zwei Masse fiir die Ausbiegung, von denen dann das Mittel genommen wurde, zu erhalten. Man wird aus der Tabelle XIV ersehen, dass die beiden Ausbiegungen sehr nahe übereinstimmten.

Bei allen Beobachtungen wurden nur Gewichte angewendet, welche hinreichten, kleine Ausbiegungen von 1-2 Cenimetern zu erzeugen, um innerhalb jener Grenzen zu bleiben, welche für die Gültigkeit der bekannten Formeln gesetzt und wo die permanenten Ausbiegungen nicht zu beträchtlich sind. Da unser Instrument die Grösse der Ausbiegungen bis auf hundcrtel von Millimetern ergab, so waren dieselben hinlänglich, um exacte Resultate zu liefern.

Es musste auch eine Fehlerquelle in Rechnung gezogen werden, die bisher vernachlässigt erscheint. Es ist diess die Zusammendriekung, welche die Unterstiitzungen immer, sobald sie einer erheblichen Belastung unterworfen werden, erleiden, auch wenn sic noch so widerstandsfähig zu sein scheinen. Diese Zusammendriickungen können von zweierlei Art sein, nämlich solche, welche mit dem Aufhören der Lastwirkung verschwinden und solche, wclche nach Wegnahme der Belastung bestehen bleiben. Bei unserer Methode die Versuche anzustellen, können nur jene ersteren die Resultate modificiren. Wir haben diese deshalb für jode Säule mit Zuhilfenahme von Marken, die auf den Lagern angebracht wurden, vorher bestimmt. Die Ortsveränderung dieser Marken unter zunehmender Belastung der Trumme wurde mit dem Kathetometer gemessen. Die Compressionen von 2 bis 3 Millimeter stellten sich als den Belastungen proportional, aber bei den zwei Stiitzpunkten etwas differirend heraus.

Die Mittelzahlen der Compressionen beider Stiitzpunkte, welche auf diese Art gefunden wurden, ergaben die Correcturen für die Ausbiegungen, Correcturen, deren relative Grösse uns nicht gestattete, sie zu vernachlässigen.

Unmittelbar nach jedem Versuch iiber die Biegung wurde die in dem Trumme enthaltene Wassermenge, durch Sägespäne bei einem transversalen Sägeschnitt, ermittelt. Diese Sägespäne, welche gleichmässig alle Jahrringe repräsentirten, wurden gemischt, 
unter der Luftpumpe getrocknet und nach der schon oben beschriebenen Methode weiter vorgegangen.

\section{C) Untersuchung der mechanischen Beschaffenheit der Hölzer in den im praktischen Leben üblichen Formen (Abmessungen).}

Dieser Theil unserer Arbeit enthält einige Versuche über Stücke von Eichen- und Tannenholz unter Bedingungen, unter denen sie im Handel vorkommen.

Wir haben uns Bohlen und Bretter, sowie Werkstiicke verschiedener Dimensionen von diesen zwei Holzarten gewählt, von schwachen Sparren (Stücke von 0.08 M. zu 0.10 M. Querschnitt) bis zu starken Balken (Stiicke von 0.23 M. zu 0.25 M. Querschnitt) mit Längen von 4 bis 7 Meter für das Eichenholz und von 10 bis 14 Meter für die Tanne. Alle diese Hölzer wurden scharfkantig hergestellt, derart, dass sie regelmässigere Prismen als die gewöhnlich angewendeten Stücke darstellten. Wir haben zuerst ihre Dichte und hierauf die Elasticitäten mittelst allmählig bis zum Bruch wachsenden Belastungen erhoben.

Um die Dichte zu finden, haben wir das absolute Gewicht, die Länge jedes Stückes und von Current- zu Currentmeter die übrigen Abmessungen genommen. Die Zahlen, welche diese letzteren in den Tabellen XVII und XVIII ausdrücken, sind die Mittelwerthe aus den abgenommenen Maassen.

Um bei den Biegungsversuchen gradatim und ohne Stösse die aufeinanderfolgenden Belastungen und mit Vermeidung jeder Gefahr zur Zeit des Bruches, anwenden zu können, haben wir in dem Apparate, dessen wir uns für die Trumme bedienten, für die Arbeitsstuicke anstatt der gusseisernen Gewichte, Belastungen durch Wasser, welche man von aussen leicht stufenweise vermehren kann, angewendet. (Tafel I Fig. 7 und 8.) Wir haben auf der entsprechend verstärkten Platte zwei zur Aufnahme des Wassers bestimmte Fässer $r$ gestellt, jedes mit einem Gehalt von 25 Hectoliter. Eine Pumpe, welche in einen ausserhalb des Mauerwerkes befindlichen Wasserlauf tauchte, diente dazu, der Tonne nach Belieben Wasser zuzuführen, von wo man es in der Folge mittelst eines am Boden angebrachten Hahnes austreten lassen konnte. Um jeden Augenblick die in den Fässern enthaltene Wassermenge erfahren zu können, haben wir einen Schwimmer in jedem Gefässe angebracht, welcher eine schon früher nach der Eichung des Fasses eingetheilte Stange tu'ug. Diese Stange reichte bis ins obere Stockwerk und zeigte, durch ihre Stellung, die man 
nach einer am Fasse befestigten Latte beurtheilen konnte, die Zahl der Litres Wasser an. Die Platte war, wie bei den vorangehenden Versuchen, durch die Winden $n$ unterstützt und an den Ketten $g$ befestiget. Da für einige kleinere Muster die vorstehende Einrichtung als erste Belastıng zu schwer gewesen wäre, so haben wir in diesem Falle nur ein Fass an den Ketten aufgehängt, und da dabei oft die Pfeilhöhen der Biegungen grösser waren, als der Lauf der Winden $n$, so ersetzten wir die Haken durch eine Schraubenwinde. Die Schraube, welche genug stark war, um unsere Belastungen auszuhalten, war am Anfang des Versuches ganz herausgedreht, und in dem Maasse, als das Fass am Niveau des Bodens ankam, wurde sie zurïckgedreht, um dasselbe wieder emporzuheben.

Die Nothwendigkeit für diese Versuche, Unterstützungen von mit der Länge der Stiicke veränderlicher Entferning zu haben, liess uns auf die gemaiuerten Stuitzen Verzicht leisten. Wir haben sie durch Pfeiler aus starken eichenen Balken, die sehr nahe aneinander gelegt wurden, ersetzt. Es war indessen nöthig, die Zusammendrückung dieser Pfeiler jeden Augenblick messen zu können. Zu diesem Ende bedienten wir uns zweier kupfernen mit Nonien versehenen Maassstäbe, welche Hundertel-Millimeter zu messen gestatteten. Diese Maassstäbe wurden von Latten $u$ getragen, die in dem Grund befestigt waren, während die Verniers an der inneren Fläche der Balken $v$, auf welchen unmittelbar die Versuchsstiicke ruhten und unter der Axe derselben angebracht waren. $\mathrm{Da}$ die oberen Theile der zu den vorangehenden Versuchen dienenden gemauerten Pfeiler weggenommen werden mussten, so haben wir an Stelle der Sicherheitsketten einen Flaschenzug $x$ gesetzt, welcher mit fixer Rolle am Bundtram des HangarDaches aufgehängt wurde. Er diente dazu, das Auflegen der Versuchsstücke zu erleichtern, die grossen Ketten aufzuheben und sie im Momente des Bruches zu halten.

Bei diesen neuen Versuchen betrug das Gewicht der Ketten und der Haken . . . . . . . . . . 1975 Kil. das der Platte . . . . . . . . . . 888.0 n das des Fasses $r$ sammt Schwimmer . . . . 4268" das des Fasses $r^{\prime}$. . . . . . . . . . . 3767

Als die Tonne $r$ allein angewendet wurde, fuigte man einen Boden aus gebolzten Bohler von dem Gewichte von 273 Kilogrammen hinzu, die Winde, welche die Haken ersetzte, wog mit ihren Bolzen 166, und die Ketten 147 Kilogramme. 


\section{$-28-$}

Der Gang der Operation ist so ziemlich derselbe, wie der oben beschriebene gewesen. Nachdem man das Versuchsstück hocilkantig auf die Stützen selegt hatte, hat man mit dem Kathetometer die Höhe des markirten Punktes und direkt die Stellung der Nonien genommen, das gab uns den in unseren Tabellen mit $o$ bezeichneten Ausgangspunkt. Hierauf hat man, je nach der Stärke der Stücke, als erste Belastung entweder blos das Gewicht der Ketten allein oder das des leeren Apparates angewendet; dann wurde die Belastung aufgehoben und alle Maasse neuerdings genommen, um die totale von der ersten Belastung erzeugte Pfeilhöhe zu bekommen. Auf gleiche Weise wurde für die verschiedenen Belastungen durch Wasser, die man erzielte, indem man die Pumpe in Thätigkeit versetzte, vorgegangen. Die bei jeder Beobachtung gefundene Ausbiegung setzt sich aus den dem betreffenden Gewichte, welches sie erzeugt hat, entsprechenden elastischen und permanenten Ausbiegungen zusammen. Die Beobachtung, welche unmittelbar nach Aufhebung der Belastung angestellt wird, ergibt die Maasse für die permanente Ausbiegung. Die Differenz der Abmessungen während und nach der Belastung ist nichts anderes, als die elastische Ausbiegung. Es versteht sich, dass die durch die Nonien gegebene Correction separat bei jeder Beobachtung vorgenommen werden muss.

Bei den dem Bruche benachbarten Belastungen wäre fiir die an den Winden manipulirenden Personen Gefahr vorhanden gewesen. Wir haben uns also damit zufriedengestellt, die totale Pfeilhöhe zu nehmen, und die letzten Belastungen haben in die zur Bestimmung des Elasticitätscoëfficienten angestellten Rechnungen nicht einbezogen werden können.

Für Latten, Bohlen, Bretter wäre als erste Belastung schon das Gewicht der Ketten allein zu gross gewesen. Wir haben deshalb (Tafel I., Fig. 9) eine Platte y, die durch die Hand mit Gewichten zu 20 Kilogrammen belastet wurde, angewendet. Sie wurde mittelst des Flaschenzuges $x$, ohne die Stiicke in Unordnung zu bringen, aufgehoben. Letztere sind durch hölzerne Stiitzen $z$, welche mit den steinernen Säulen verbolzt waren, getragen worden, welche zusammen im Vergleich zu den angewendeten Belastungen so stark gebaut waren, dass wir geglaubt haben, in diesem einen Falle die Zusammenpressung der Stützen vernachlässigen zu dürfen.

Die Bestimmung dieser Zusammendrïckungen hätte ja schliesslich nur zu, im Vergleich mit den erlangten Ausbiegungen 
bedentungslosen Correcturen geführt. Die Bretter und Bohlen sind anstatt hochkaniig gestellt zu werden auf ihre Breitseite gelegt worden, im Uebrigen war der Gang der Operation derselbe.

Bei keiner der Beobachtungen dieser letzten Serie haben wir die in den Hölzern enthaltene Quantität Wasser gesucht, weil sich dieselben in dem Zustande der gewöhnlich angewendeten befanden, für welche wir schon durch Specialversuche die Coëfficienten der durch dic Feuchtigkeit hervorgerufenen Veränderungen gefunden hatten. 


\section{DRITTER THEIL.}

\section{Calcul der Versuche und Besprechung der Methoden.}

Nachdem wir die angewendeten Methoden und Apparate beschrieben, so ist es jetzt unsere Aufgabe die Formeln anzuführen, welche zur Berechnung und Vergleichung der nach den verschiedenen Verfahrungsweisen gewonnenen Resultate gedient haben.

Was die Dichte anbelangt, so wird man bemerken, dass die mittelst des Volumens eines ganzen Trummes gewonnenen Werthe im allgemeinen grösser sind, als die aus den Stäben oder Schichten, welche dasselbe Trumm zusammensetzen, abgeleitet wurden. Dieser Unterschied kann zwei Ursachen zugeschrieben werden. Um die Werthe untereinander vergleichbar zu machen, war es unerlässlich, sie auf den gleichen constanten Feuchtigkeitsgehalt zurückzuführen. Wir haben den von $20 \%$ gewählt. Die zu dieser Reduction dienenden Coëfficienten sind, da sie nur für Stäbe, deren Feuchtigkeitsgehalt zwischen circa 8 und 40 zu 100 schwankt, bestimmt werden konnten, möglicherweise nicht constant für beträchtlichere Feuchtigkeitsunterschiede oder lassen sich nicht genau auf jene der Trumme in Anwondung bringen. Andererseits kann die Bestimmung der Dichte der Trumme mittelst der Dichte der Stäbe deshalb nicht scharf sein, da die Stäbe nicht die Totalität der Jahresschichten der Walzen repräsentiren.

Wie wir schon oben angegeben haben, wurde zur Untersuchung der elastischen Beschaffenheit der Stäbe zweierlei angewendet, die Methode der Longitudinal-Schwingungen und jene der Verlängerungs-Ausdehnung. Die erstere gibt direct die Schall- 
geschwindigkcit, die zweite den Elasticitätscoëfficienten. Diese beiden Grössen sind durch die Gleichung

in welcher

$$
E=\frac{v^{2} \cdot d}{g}
$$

$E$ der Elasticitätscoëfficient,

$v$ die Schallgeschwindigkeit und

$d$ die Dichto ist,

in Verbindung gebracht.

Wenn man als Einheit für die Lünge, den Millimeter, für die Gewichte das Kilogramm, für die Schallgeschwindigkeit jene in der Luft, für die Dichte das specifische Gewicht des Wasser's annimmt, so hat man

$$
\log \mathrm{E}=2 \log \mathrm{v}+\log \mathrm{d}+1 \cdot 05130 \quad \text { 1) }
$$

Man kann nun mit Hülfe dieser Formel den Elasticitätscoëfficienten aus den Longitudinal-Schwingungen und umgekehrt aus der Verlängerung die Schallgeschwindigkeit ableiten.

Man weiss aber für die Gase und Metalle, dass die aus den Longitudinal-Schwingungen berechneten Ziffern für E grösser als die aus der Ausdehnung oder Zusammendrückung gefundenen sind. Wir haben deshalb geglaubt untersuchen zu sollen, ob sich die Hölzer ebenso verhalten. Eine Berichtigung dieser Art war nicht blos vom theoretischen Standpunkt aus interessant, sondern sie war nothwendig, um die mit einer oder der anderen der beiden Methoden erhaltenen Resultate untereinander vergleichbar zu machen.

Wir haben in die Tabelle Nr. VII die erhaltenen Ergebnisse und das Verhältniss zwischen den beiden Elasticitätscoëfficienten und den beiden Schallgeschwindigkeiten für alle Stäbe, welche vergleichsweise beiden Methoden unterworfen wurden, eingetragen. Das erste dieses Verhältnisses ist offenbar das Quadrat des zweiten. Wenn man diese Tabelle durchgeht, so wird man sehen, dass die Schwingungen auch für die Hölzer zu höheren Ziffern führen, als die Ausdehnungen, und dass die Trockenheit ohne Linfluss auf die Grösse der Beziehungen erscheint. Wir haben demnach als Correctionscoëfficienten die Mittelzahlen der für die Stäbe eines Trummes bei verschiedener Feuchtigkeit gefundenen Verhältnisse annehmen kömen. Um aber den Elasticitïtscoëfficienten, den wir den wirklichen (reellen) nennen, das ist derjenige, den die Ausdehnung ergeben hatte, zu erhalten, muss man den aus den Schwingungen abgeleiteten Elasticitätscoëfficienten durch den ersten jener Coëfficienten dividiren, während man die aus der Ausdehnung abgeleitete Schallgeschwia- 
digkeit mit dem zweiten multipliciren muss, um die wirkliche Schallgeschwindigkeit zu bekommen. Diese Correctionscoëfficienten sind für die Trumme derselben Holzarten nahezu dieselben.

Dieselben Coëfficienten darf man auch auf die durch Transversalschwingungen erhaltenen Resultate anwenden, denn bekanntlich sind diese mit den aus Longitudinalschwingungen hervorgehenden gleichwerthig. Man leitet also den wirklichen Elasticitätscoëfficienten $\mathrm{ab}$ mit der Formel

$\log \mathrm{E}=\log \mathrm{p}+2 \log \mathrm{n}+3 \log \mathrm{l}-3 \log \mathrm{a}-\log \mathrm{b}-$ $4 \cdot 02104-\log \mathrm{c}$

in der $p$ das absolute Gewicht des Barrens,

$l$ seine Länge,

$n$ die Anzahl der Doppel-Transversalschwingungen per Secunde,

$a$ die Seite des Querschnittes, welcher die Schwingungen folgten,

$b$ die andere Seite,

$c$ der Correctionscoëfficient für die Elasticitätscoëfficienten jenes Trummes, dem der Barren entnommen war. Um die wirkliche Schallgeschwindigkeit zu finden, muss man in die Formel 1 den nicht corrigirten Werth von $\log E$ substituiren.

Wir haben schon auseinandergesetzt, wie wir darauf gekommen sind, zur Bestimmung der Eigenschaften ganzer Bäume und für die Vergleichung der verschiedenen Arten, cylindrische Trumme von nur 2 Meter Länge anzuwenden. Diese Anordnung gestattet mit Stücken von regelmässiger Form in ihrem natürlichen $\mathrm{Zu}$ stande und bei Erhaltung aller Jahrringe zu arbeiten; aber andererseits herrschen nicht mehr die Umstände, für welche die Formeln aufgestellt wurden. In der That sind diese Formeln streng genommen nur auf vollkommen homogene Stiicke anwendbar, während in den Bäumen die Elasticität, wie wir dies wohl für jedes Trumm erkennen konnten, von Jahrring zu Jahrring gesetzmässig variirt, welches Gesetz, wie wir später sehen werden, mit dem Alter und der eigenen Natur der Bäume wechseln kann. Ueberdiess sind diese Formein blos für Walzen gültig, deren Durchmesser im Verhältniss zu ihrer Länge unbeträchtlich ist und nicht für starke Durchmesser, wie wir sie im Lanfe unserer Untersuchungen begegnet haben. Wir haben uns denn, um uns so wenig als möglich von den Bedingungen des Problemes zu entfernen, darauf beschränken müssen, nur sehr kleine Ausbiegungen hervorzurufen. Wir haben ferner für eine gewisse 
Anzahl von Trummen, die bei der Biegung gewonnencin Legelb. nisse durch Vergleich mit den bei der Ausdchnung der Stabe, (die aus den verschiedenen Jahrringen derselben Trumme gonommen wurden) erhaltenen, controllirt.

Die mittleren Elasticitätseoëfficienten wurden für die Trumme aus den Ausbiegungen mittelst der gewöhnlichen Formel

abgeieitet, in welcher

$$
\mathrm{E}=\frac{2 \mathrm{P} \cdot \mathrm{a}^{3}}{3 \pi \mathrm{f} \cdot \mathrm{r}^{4}}
$$

$P$ die Belastung in Kilogrammen,

$a$ die Hälfte der Entfernung der beiden Stützen,

$f$ die elastische Pfeilhöhe,

$v$ den Halbmesser des Trummes bedeutet.

Wir haben die so gewonnenen und auf $20 \%$ Feuchtigkcit reducirten Coëfficienten in dic Tabelle Nr. XVI eingetragen, an (ier Seite derjenigen, welche wir in der Art, wie wir diess später auscinander setzen werden, aus der Verlängerung der Stäbe für die ganzen Trumme berechnet haben. Man sieht aus dieser Tabelle, dass die Ziffern, abgesehen von einigen Abweichungen, hinreichend iibereinstimmen, um im allgemeinen diesen Vorgang einhalten zu kömuen, namentlich wenn es sich nicht um die Vergleichung von Bäumen derselben Art handelt. Dieses Resultat wurde noch durch jenes bestätiget, welches für die aus verschiedenen Höhen bei ein und demselben Baume genommenen Trumme dadurch gewonnen wurde, dass man die einen der Biegung, die anderen der Ausdehnung unterworfen hatte. Die erhaltenen Ziffern folgen derselben Progression und zeigen nur Abweichungen von solcher Grösse, dass sie der Höhe im Bamme zugeschrieben werden kïnnen.

Nur die harzreichen Bäume zeigen eine merkwürdige Anomalie. Alle von der Ausbiegung der Trumme herstammenden Coëficienten, namentlich jene von Trummen, deren Durehmesser sehr gross war, erscheinen viel zu klein, wemn man sie mit den dureh Ausdehnung gewonnenen Ziffern vergleicht, ja selbst im Verhältniss zu den aus Biegungsversuchen nuit viereckigeu Stiicken von hinreichender Länge hervorgehenden. Es ist also evident, dass die weiter oben angefuihrte Formel sich nicht auf Valzen aus solehen Stoffen anwenden lïsst. Es lïsst sich diess vielleicht einem Mangel an hinreichender Adhärenz und gegenseitiger liückwirkung der Holzschichten untereinander und dem Umstande zuschreiben, dass sich (wie mam bald sehen wird) die festesten Jahrringe am Unfange vorfinden. 
Wir haben demnach in unsere allgemeinen Mittelzahlen die Ergebnisse der Biegungsversuche mit Walzen von Nadelhölzern nicht einbezogen, wohl haben wir aber von denselben alle Elemente gegeben, um, wenn man später daran geht die Formeln zu modificiren, von ihnen genaue Resultate erwarten zu können.

Für die grossen Eichen- und Tannenstücke in den im Handel üblichen Formen haben wir uns der gewöhnlich angewendeten und schon hinreichend bewährten Formeln bedienen können. Wir haben den Elasticitätscoëfficienten aus der mittleren Pfeilhöhe, welcher aus einer genügend grossen Anzahl von Messungen bestimmt wurde, nach der Formel

$$
\left.\mathrm{E}=\frac{2 \mathrm{P} \cdot \mathrm{a}^{3}}{\mathrm{f} \cdot \mathrm{b} \cdot \mathrm{c}^{3}} \quad 3\right)
$$

abgeleitet. In derselben ist $b$ die Breite und $c$ die Höhe, $d$. h. die Dimension des Stückes, in deren Sinn die Last wirkt. In den Formeln 2 und 3 ist auf das Eigengewicht der Versuchsstïcke keine Rücksicht genommen, weil dieses Gewicht als ein Theil der permanenten Belastung erscheint, nachdem die Pfeilhöhen durch Subtraction gewonnen wurden.

Der Widerstand bei dem Bruch per Quadratmillimeter Querschnitt wurde für diese Stücke nach der Formel

$$
\mathrm{R}=\frac{3\left[\mathrm{P}\left(\mathrm{a}^{2}+1.5 \mathrm{f}^{2}\right)+\pi\left(\mathrm{a}^{2}+3 \mathrm{f}^{2}\right)\right]}{\mathrm{abc}^{2}}
$$

berechnet, in welcher $P, a, b, c$ und $f$ dieselben Bedeutungen wie in den Formeln 2 und 3 haben, und unter $\pi$ das Gerwicht des zwischen den Unterstützungspunkten liegenden Theils des Stückes verstanden wird, welches letztere aus dem Gewicht und der ganzen Länge des Stückes und der Entfernung der Stützpunkte leicht berechnet werden konnte.

Die mittlere Festigkeit für alle Holzarten wurde endlich durch Abreissung der Stäbe bestimmt und man wird sehen, dass sie für die Eiche und Tanne mit den Werthen, welche durch Bruch bei Ausbiegung gefunden wurden, gut übereinstimmen. 


\section{VIERTER THEIL.}

\section{Besprechung der Resultate.}

Wir haben am Anfang dieser Abhandlung den Weg angegeben, welcher uns als der natürlichste erschien, und den wir desshalb zum Behufe des Studiums der mechanischen Eigenschaften des Holzes zu verfolgen uns vorgenommen hatten.

Vor allem hatten wir zu untersuchen, wie sich die Hölzer, wenn sie von aussen einwirkenden Kräften unterworfen wurden, verhielten und nach welchen Gesetzen die Formveränderungen Platz griffen. Denn es wäre möglich, dass diese Formveränderungen nicht den angewendeten Kräften proportional wären und dass deshalb die gewöhnliche Definition des Elasticitätscoëfficienten nicht anwendbar gewesen wäre. Ueberdies hätten die ersten Belastungen schon in den Fasern Veränderungen hervorrufen können, welche genügt hatten, um die Eigenschaften der Hölzer und die für sie geltenden Gesetze zu modificiren. Wir konnten demnach erst dann untersuchen, wie die mechanischen Eigenschaften in den verschiedenen Theilen eines und desselben Baumes variiren, nachdem wir uns überzeugt hatten, ob die bekannten Gesetze und Formeln sich auf die Hölzer anwenden liessen.

Die erste Frage, die sich uns dann darbot, war: sich zu versichern, ob die Lage (Orientation) der verschiedenen Theile eines Baumes wirklich auf ihre Eigenschaften Einfluss nimmt, und ob man sich diese Ueberzeugung bei den in ein und demselben Jahrring, aber in verschiedener Lage gegen die Weltgegenden gewählten Stäben verschaffen konnte, bevor man den Einfluss der Quantität des hygrometrischen Wassers kannte, allerdings vorausgesetzt, dass man nur unter gleichen Bedingungen genom- 
mene Stïbe in Anwendung brachte. Es war demnach, um die Ergebnisse aller Versuche dadurch vergleichbar zu machen, dass sie auf einen bestimmten Feuchtigkeitsgrad reducirt wurden, unerlïsslich, diesen Einfluss der Fenchtigkeit auf das Volumen und die übrigen Eigenschaften zu bestinmen und durch Coëfficienten auszudrücken.

Wir hatten bei diesen Versuchen den Gang der mechanischen Eigenschaften in den vom Centrum verschieden entfernten Jahrringen, in denselben. Jahrringen bei verschicdenen Höhen und endlich in den verschiedenen Richtungen zu studiren und für die Bäume derselben Gattung den Einfluss des Alters, der Exposition und der Natur des Bodens zu untersuchen.

Die mechanischen Eigenschaften wurden so für jede $\mathrm{Holz}$ art bestimmt; wir hatten aber nun die Relationen, die unter ihnen bestehen konnten, aufzustellen und die praktischen aus unserer Arbeit hervorgehenden Consequenzen abzuleiten.

In den dieser Abhandlung angehängten Tabellen findet man alle Daten unserer Versuche, sowie die Resultate des angewendeten Calculs. Wir waren, wie man sehen wird, angewiesen für jede der Fragen, die wi" uns gestellt haben, eine grosse Zahl von Versuchen anzustellen, und zwar hauptsächlich mit den, in der Lokalität, wo wir arbeiteten, gewöhnlichsten Holzarten. In der That können sich die Gesetze durch Untersuchungen, die an einem einzelnen Individuum vorgenommen werden, nicht mehr bestimmen lassen, sobald man es mit organisirten Körpern zu thun hat, welche immer Abweichungen in ihrem Bau darbieten. Speciell bei den Hölzern sind die Unregelmässigkeit im Wachsthum, die Veränderungen in der Breite und dem Verlanfe der Jahrringe, welchen man oft begegnet, die Astknoten und andere Umstände, die sich nicht voraussehen, nicht einmal immer nachweisen lassen, das alles sind Veranlassungen zu Unregelmässigkeiten in den Ziffern. So haben wir auch, olne uns bei eigenthümlichen Ausnahmen aufzuhalten, aus der Gesammtheit der Fälle die Gesetze zu erkennen suchen müssen.

Wir hoffen diess in einer geniigenden Weise für die Mehrzahl der Fragen über dic Unterschiede in den Individuen und in den Arten erreicht zu haben, aber wir haben nicht vollständig die Fragen bezüglich der örtlichen Einflïsse entscheiden können, welche noch viel zahlreichere und in verschiedenen Klimaten, sowie an verschiedenen Oertlichkeiten angestellte Versuche erfordert hätten. 


\section{$-\quad 37$}

Nach dieser kurzen Auscinandersetzung der Betrachtungen, wolche uns geleitet haben, eriibrigt uns die Resultate, zu denen wir gelangt sind, anzugeben und zu besprechen.

I. Man sielit aus den in die Tabelle $\mathrm{Nr}$. IV eingetragenen Ausdehnungsversuchen (Verlängerung, Streckung), dass man bei den Hölzern, wie bei den Metallen zwei Arten von Ausdehnungen, die elastische Ausdehnung und die permanente Ausdehnung, unterscheiden kann und dass die erstere den Belastungen sehr erkennbar proportional ist. Die erste der gemessenen Ausdehnungen ist manchmal um ein geringes nicht übereinstimmend mit den anderen gewesen, wiewohl wir besorgt waren, die Stäbe ror der ersten Messung mit einem Gewicht zu belasten, das gerade hinreichte, um sie gerade zu richten und das folglich nicht in jener Last, die die elastische Ausdehnung hervorbrachte, eingerechnet wirde. Uebrigens tritt derselbe Fall bei den Metallen ein.

Was die permanenten Ausdehnungen anbelangt, so sieht man, dass sie in einer unregelmässigen Art mit den Belastungen, von den ersten angefangen, wachsen, was sich leicht daraus erklïrt, dass sie eben nicht blos von der Grösse der Belastung, sondern auch von der Daucr derselben und von der Art zu operiren abhängen. In einer gewissen Anzahl von Fällen verringern sich gar nach mehreren Belastungen diese Ausdehnungen, anstatt sich zu vergrössern, um in der Folge wieder ihren gewöhnlichen Gang zul nchmen. Diese Art von Rücksprüngen, welche auch bei den Metallen, wenn auch minder häufig, auftreten, scheinen einer heftigen und unregelmässigen Zusammenzichung der Fasern, zufolge der Wegnahme der Gewichte zuzuschreiben sein. Aus diesen Betrachtungen erhellt, dass mann. der Theorie nach einen bestimmten Werth weder für die Elasticitïtsgrenze, noch für die Maximalausdehnung wiirde aufstellen können. Auch haben wir diese Daten bei unseren Versuchen nicht speciell gesucht. Jedenfalls kann man sie nach der gewöhnlichen Annahme aus den gemessenen Ausdehnungen ableiten, und wir haben Sorge getragen, Mittelwerthe für dieselben in unseren Schlussfolgerungen zu geben.

Die Tabellen Nr. XVII und XVIII zeigen, dass dieselben Gesetze für die der Biegung unterworfenen Stiicke giltig sind. Die permanenten Ausdehnungen sind in der Columme der corrigirten totalen Ausdehmungen enthalten. Um die Proportionalitit zwischen den Pfeilhöhen und den correspondirenden Belastungen ersichtlich zu machen, haben wir alle Versuche auf eine bestimmte Last von 100 Kilogrammen zurückgeführt. Und die so durch die 


\section{$-38-$}

Rechnung gefundenen Pfeilhöhen stimmen genügend überein, um sich ihrer Mittelwerthe zur Berechnung der Elasticitätscoëfficienten bedienen zu können.

II. Die Stäbe, welche wir aus ein und demselben Jahrringe, aber aus den den vier Weltgegenden gegenüberliegenden Theilen eines Baumes (Tabelle Nr. VIII), genommen haben, zeigten in den Unterschieden ihrer mechanischen Eigenschaften keinerlei Regelmässigkeit. Ja für die verschiedenen Jahrringe desselben Trummes fanden sich die Maxima und Minima ebenso sehr in der einen, wie in der anderen der Richtungen. Diese Unregelmässigkeit war in allen Fällen derart constant, dass wir, nachdem wir 14 verschiedene Trumme von Eichen, Rothbuchen, Akazie, Tanne und Fichte geprüft hatten, es für unnütz hielten, diese Untersuchung weiter zu treiben. Auch haben wir deshalb, als es sich darum handelte, den Gang der mechanischen Eigenschaften in den Jahrringen dieser Walzen zu verfolgen, die Mittelwerthe der so gefundenen vier Werthe für die Jahrringe in Verwendung gebracht.

Für zwei dieser Walzen, Eiche und Rothbuche, haben wir nicht blos die Stäbe den Ausdehnungsversuchen unterworfen, sondern wir haben unsere Resultate durch die Methode der Ausbiegung an Cylindern von hinreichend starkem Durchmesser und einer Mittelstellung (Tafel I, Fig. 2), nämlich aus den gegen Nordost, Nordwest, Südost und Südwest exponirten Theilen, controllirt.

Die in die Tabelle Nr. XIV eingetragenen Ergebnisse dieser letzteren Versuche bestätigen jene durch Ausdehnungsversuche erhaltenen Punkt für Punkt.

III. Wir haben in der Tabelle Nr. IX die Resultate aller Versuche über den Einfluss der Feuchtigkeit vereinigt. Alle Stäbe, mit denen wir gearbeitet haben, sind in drei oder vier verschiedenen Zuständen untersucht worden und die Tabelle stellt die Jahrringe, denen sie angehörten, nach ihrer Entfernung vom Centrum und nach ihrem Feuchtigkeitsgrade geordnet, dar. Wir müssen neuerdings bemerken, dass, wie wir schon weiter oben auseinandergesetzt haben, ein und dieselben Stäbe nicht in allen Feuchtigkeitsgraden verwendet werden konnten, und dass wir, um genügend zahlreiche Vergleichspunkte zu haben, gezwungen waren, die unmittelbar nebeneinander aus dem gleichen Jahrringe genommenen Stäbe als identisch anzusehen. Diese Identität kann jedoch nicht jederzeit eine vollständige gewesen sein, und dieser Umstand erklärt die Anomalien, denen man zuweilen in der Tabelle begegnet. 
Die Dichte nimmt bekanntlich im allgemeinen mit der Quantität des enthaltenen Wassers ab. Diese Abnahme ist in allen Fällen, wo ein grosser Unterschied in den Feuchtigkeitsgraden obwaltet, sehr merklich. Die Differenzen in der Dichte, die man dann findet, sind hinreichend gross, um durch die Fehlerquellen und die schon signalisirten Unregelmässigkeiten nicht maskirt zu werden. Auch ist zu bedauern, dass wir, um die Jahrringe selbst vergleichen zu können, gezwungen waren nur mit sehr schwachen Stäben zu arbeiten. Solche Stäbe trocknen an der Luft sehr schnell, und davon rührt es her, dass fast nie eine grössere Abweichung als 10 oder 20 Percent zwischen den trockensten und feuchtesten Stäben zum Vorschein kam. Die Schwierigkeit prëciser Bestimmungen innerhalb so naher Grenzen und besonders in jenen Fällen, wo wir mit kleinen an den Enden der Stäbe genommenen Stücken arbeiteten, erklärt die zahlreichen Anomalien, denen wir bei der Dichte und hauptsächlich in den äussersten Jahrringen harzreicher Hölzer begegnet sind. Aber trotz dieser Anomalien macht der allgemeine Gang der Ziffern nicht nur die Abnahme der Dichte im Gefolge der Austrocknung des Holzes, sondern selbst deren Proportionalität erkenntlich.

Um dieses Gesetz aufzustellen, welches wir weit entfernt sind als streng genau hinzustellen, haben wir die Dichten bei den verschiedenen Graden der successiven Austrocknung mit der Dichte beim höchsten Feuchtigkeitsgrade verglichen.

Bezeichnen wir mit $h$ die in Percenten des absoluten Holzgewichtes ausgedrückte Feuchtigkeit, mit $d$ die entsprechende Dichtc. Es seien ferner $h^{\prime}$ und $d^{\prime}$ dieselben Quantitäten für einen geringeren Feuchtigkeitsgrad und endlich $c$ der Abweichungscoëfficient der Dichte für 1 Percent Feuchtigkeit, so wird man haben

$$
\begin{aligned}
& d^{\prime}=d\left[1-c\left(h-h^{\prime}\right)\right] \\
& \text { woraus } c=\frac{d-d^{\prime}}{d\left(h-h^{\prime}\right)}
\end{aligned}
$$

Wir haben für ein und denselben Jahrring für verschiedene Feuchtigkeitsgrade hinreichend nahe liegende Werthe für $c$ gefunden, auf dass wir das Mittel nehmen konnten (wobei wir jederzeit jene, wo der Feuchtigkeitsunterschied 1 Percent nicht überstieg, wegliessen), und haben diese für jeden Jahrring derart gefundenen Mittelzahlen in die Tabelle Nr. X eingetragen. Es wurden dabei mit dem Zeichen + die Ausnahmsfälle, nämlich jene, in denen sich die Dichte vermehrt, statt vermindert, be- 
zeichnet. In diesem Falle wird die weiter oben gegebene Formel folgende Form erhalten:

$$
\mathrm{d}^{\prime}=\mathrm{d}\left[1+\mathrm{c}\left(\mathrm{h}-\mathrm{h}^{\prime}\right)\right]
$$

Die Werthe von $c$ variiren erheblich für die verschicdenen Jahrringe. Es ist uns indessen rationell erschienen, Mittelwerthe von allen für dieselbe Holzart von uns gefundenen Coëfficienten, als eine in den Rechnungen für den ganzen (ein und demselben) Baum anwendbare Ziffer, aufzustellen.

Die Schallgeschwindigkeit nimmt, wenn man mit ein und denselben Stäben arbeitet, immer mit der Trockenheit zu. Ein eclatantes Beispiel hiefür findet man in den von den sechs Trummen dor Tanne Nr. 56 (Tabelle Nr. IX) herrïhrenden, welche nicht eine einzige Ausnahme von diesem Gesetz darbieten. Die Ausnahmen, welche sich fuir die anderen Trumme in der Tabelle finden, kommen fast alle davon her, dass zwei Serien von Stäben vorhanden waren, und sie entfallen, wenn man dafür Sorge trägt, dass nur die Stäbe einer Serie untereinander verglichen werden. Die crste Serie umfasst jene Stäbe von grünem und von an der Sonne getrocknetem Holz, die zweite enthielt die im Laboratorium und die im Ofen getrockneten Stäbe. Diese Vergrösserung der Schallgeschwindigkeit ist merklich dem Verlust an Wasser proportional. Wir haben hiefür die Coëfficienten bei einer Trockenheitszunahme von 1 Percent durch einen Calcul bestimmt, der jenem analog ist, dessen wir uns für die Coëfficienten der Veränderungen der Dichte bedient haben.

Es seien $v$ und $v$ die Schallgeschwindigkeiten bei den Feuchtigkeiten $h$ und $h^{\prime}$, vorausgesetzt, dass $h^{\prime}>h$, es sei ferner $c^{\prime}$ der Coëfficient der Veränderung, der Schallgeschwindigkeit, so wird man haben:

$$
\mathrm{v}^{\prime}=\mathrm{v}\left[1+\mathrm{c}^{\prime}\left(\mathrm{h}-\mathrm{h}^{\prime}\right)\right]
$$

Wir haben mittelst dieser Formel die verschiedenen Werthe von $c^{\prime}$ gerechnet und haben sie für denselben Jahrring, für verschiedene Feuchtigkeitsgrade, im allgemeinen constant gefunden. Wir haben nun, wie bei den Dichten, von denselben die Mittelwerthe genommen, welche wir dann ebenso als die allgememen Mittelwerthe furr ein und dieselbe Holzart in die Tabelle $\mathrm{Nr}$. X eingetragen haben.

Was den Einfluss der Fenchtigkeit auf den Werth des Elastisitätscoëfficienten anbelangt, so haben wir bei der Besprechung der Methoden die Formel angefuihrt, mittelst deren man diesen Coëfficienten ans der Dichte und der Schallgeschwindigkeit ableitet. und wir haben uns desselben, um in jedem Fall für die 
verschiedencen in der Tabelle Nr. IX eingetragenen Fenchtigk ritsgrade die Coëfficienten der Elasticität zu rechnen, bedient.

Diese Formel ist

$$
\mathrm{E}=\frac{\mathrm{d} \cdot \mathrm{v}^{2}}{\mathrm{~g}}
$$

Ist dann der Elasticitätsenëfficient $E$ bei einer bestimmten Feuchtigkeit $h$ gegeben, so wird man den Coëfficienten $E^{\prime}$ für cine geringere Feuchtigkeit $h^{\prime}$ finden mittelst der Formel

und wenn $\operatorname{man} h \stackrel{\mathrm{g}}{-} h^{\prime}=H$ setzt, wird

$$
\mathrm{E}^{\prime}=\frac{\mathrm{d} \cdot \mathrm{v}^{2}}{\mathrm{~g}}\left[1-\mathrm{c}\left(\mathrm{h}-\mathrm{h}^{\prime}\right)\right]\left[1+\mathrm{c}^{\prime}\left(\mathrm{h}-\mathrm{h}^{\prime}\right)\right]^{2}
$$

$$
\mathrm{E}^{\prime}=\mathrm{E}(1-\mathrm{c} \mathrm{II})\left(1+\mathrm{c}^{\prime} \mathrm{H}\right)^{2} \text {; }
$$

worin $c$ und $c^{\prime}$ schon für jede Holzgattung in der Tabelle $\mathrm{Nr}$. X gegeben sind.

Diese Gleichung gibt den mit der Feuchtigkeit zunchmenden Gang des Elasticitätscoëfficienten.

Im Allgemeinen ist nach den aus unseren Versuchen hervorgehenden Werthen $c^{\prime}>c$ oder in äussersten Falle $c^{\prime}=c$, so wie diess sehr auffalend für die Esche gilt. In diesen Fällen wird der Coëfficient constant mit $H$, das ist mit der Austrocknung zunehmen.

Setzt man wirklich $c=c^{d}$ in unserer Gleichung, so wird

$$
\mathrm{E}^{\prime}=\mathrm{E}\left[1+\mathrm{cH}-(\mathrm{cH})^{2}-(\mathrm{cH})^{3}\right]
$$

Da aber aus den für $c$ und $c^{\prime}$ gefundenen Werthen und aus dem Umstande, dass $H$ seiner Natur nach nicht grösser als 60 oder 70 sein kann, hervorgeht, dass $c H$ immer ein echter Bruch sein wird, so folgt, dass die Werthe von $E^{\prime}$ constant mit jenen von $H$ wachsen werden und das umsomehr, wenn $c<c^{\prime}$ ist.

Es gilt aber nicht dasselbe, wenn $c>c^{\prime}$ ist, ein Fall, den wir bei der Tamne angetroffen haben. Der Gang des Coëfficienten wird dann abhängen von dem Verhältniss von $c$ zu $c^{\prime}$. Wenn man in die Formel die Werthe dieser beiden Coëfficienten substituirt, so findet man, dass $E^{\prime}$ fiir $H=23 \cdot 21$ ein Maximum erreicht. Aber bevor man diese Folgerung aus der Formel einführt, würde es nothwendig sein, dieselbe durch specielle Versuche mit langsam von hundertel zu hundertel zu trocknenden IIölzern bestiitigen zu lassen.

Wenn $c=2 c^{\prime}$ ist, ein Fall, der bei den von uns untersuchten Holzarten nie vorkam, so würde man haben:

$$
\mathbf{E}^{\prime}=\mathrm{E}\left[1-\ddot{3}\left(\mathrm{c}^{\prime} \mathrm{H}\right)^{2}-2\left(\mathrm{c}^{\prime} \mathrm{H}\right)^{3}\right]
$$

was so viel bedeutet, als: die Elasticitiat wiirde mit dem Grade der 'Trockenheit beständig abnehmen. 


\section{- 42}

Dic Festigkeit oder der Widerstand gegen den Bruch durch Ausdehnung (Zug) vermehrt sich in Folge der Trocknung, und zwar in einem genügend starken Verhältniss in fast allen Fällen (siche Tabelle Nr. IX). Nur wenn die Trocknung so weit getrieben wurde, dass sie blos 10 Percent Feuchtigkeit zurückliess, haben sich sehr charakteristische Verminderungen der Festigkeit gezeigt. Es wäre schwierig zu entscheiden, ob diese Verminderungen von einer durch die Wärme erzeugten Störung in den Fasern oder in ihrer Textur herrühren, oder ob man sie dem Umstande zuschreiben muss, dass die sehr trockenen Hölzer eine permanente Verlängerung fast nicht mehr annehmen und dann so spröde und zerbrechlich werden, um unter dem geringsten Stoss oder senkrechten Anprall, auf die Richtung der Belastung zu Grunde zu gehen. Die allgemeine Annahme der Ansicht von der grössten Festigkeit des grünen Holzes, die Ansicht, welche von jenen Autoren, die das Holz nur der Biegung unterworfen haben, ausging, erklärt sich auch aus dem oben erörterten Umstand. Wir haben geglaubt die Coëfficienten der von uns allgemein beobachteten Vergrösserung der Festigkeit nicht bestimmen zu sollen, da die Versuche über den Bruch, wie wir schon oben bemerkt haben, zu wenig Genauigkeit zulassen, um einen Calcul dieser Art zu gestatten.

Es hat uns nützlich geschienen von den bei verschiedenen Feuchtigkeitsgraden für die Stäbe gefundenen Dimensionen, unabhängig von den mechanischen Eigenschaften, welche wir in allen Partien unserer Arbeit studirt haben, Nutzen zu ziehen, und zwar zur Bestimmung ihrer transversalen Zusammenziehung (Schwindung) in Folge der Austrocknung. Zu diesem Behufe haben wir bei demselben Trockengrade die Mittelwerthe der Seiten der quadratischen Querschnitte aller demselben Trumme angehörenden Stäbe genommen. Ihr Verhältniss untereinander hat uns dann die mittleren Contractionscoëfficienten für den Verlust eines Procentes Feuchtigkeit ergeben. Diese Coëfficienten und die Dimensionen der Seiten, von denen sie abgeleitet wurden, sind in der Tabelle Nr. XI enthalten. Wir hatten die Mittelzahlen für jedes Trumm und jede Holzart im Auge gehabt. Aber wie wir manchmal für die verschiedenen Trumme ein und desselben Baumes grössere Abweichungen gefunden haben, als bei der Mehrzahl jener der verschiedenen Holzarten untereinander, so haben wir die allgemeine Nittelzahl von allen beobachteten Fällen genommen, und diese Mittelzahl sollte, wie uns scheint, so lange als Erfah- 
rungsziffer angewendet werden, bis neue, specielle und zahlreichere Versuche in dieser Hinsicht gemacht worden sein werden.

IV. Die Untersuchungen, welche wir jetzt besprochen haben, liaben uns in die Lage versetzt, unsere sämmtlichen Versuche, indem sie auf einen und denselben Trockenheitsgrad zuriickgeführt wurden, untereinander vergleichbar zu machen. Als entsprechendster Fixpunkt erschien uns die Feuchtigkeit von 20 Percent, diese ist diejenige, zu welcher die Hölzer bei natülicher Trocknung in der Regel nahezu gelangen. Um diese Reduction durchzuführen, haben wir die Dichten und die Schallgeschwindigkeiten durch Interpolation zwischen die beiden über und unter 20 Percent nächstliegend gefundenen Feuchtigkeiten ermittelt. Wenn wir keine Beobachtung oberhalb von 20 Percent hatten, so haben wir jene, welche sich am meisten nähcrte und eine zweite von dieser nur um einige Hundertel differirende genommen. Wir hatten also in jedem. Falle vier Becbachtungen, nämlich zwei Dichten $d$ und zwei Schallgeschwindigkeiten $v$. Wenn man diese Werthe in die Interpolationsformel

$$
d=a+b \cdot h \text { und } v=a^{\prime}+b^{\prime} h
$$

substituirt, so kann man die vier Constanten bestimmen und $h=20$ gesetzt findet man die gesuchte Dichte und Schallgeschwindigkeit. Wir haben es vorgezogen, diesen Weg zu verfolgen, als uns der früher nach allen Versuchen bestimmten mittleren Coëfficienten zu bedienen. Es war in der That, da es sich nicht um den allgemeinen Gang der Austrocknung, sondern um Aufsuchung einer speciellen auf einen einzelnen Feuchtigkeitsgrad anwendbaren Ziffer handelte, viel richtiger, statt der entferntesten Grenzen, die dem Fixpunkt zunächst gelegenen Beobachtungen zu nehmen.

Die Elasticitätscoëfficienten wurden aus den so für die Dichte und Schallgeschwindigkeiten gerechneten Werthen gefunden; nur haben wir noch, da es sich nicht darum handelte, die aus den Vibrationen sich ergebenden Coëfficienten, sondern jene, die aus den Ausdehnungen hervorgehen, und welche wir die "wirklichen" genannt hatten, zu erhalten, auf diese ersteren die durch die Tabelle Nr. VII gegebenen Correctionscoëfficienten angewendet.

Die Festigkeiten sind ebenfalls durch Interpolation gerechnet worden. Wenn für einen Jahrring nur Beobachtungen bei einem Feuchtigkeitsgrad vorhanden waren, so hat man die Veränderungscoëfficienten für die anderen Jahrringe ebenso gesucht, wie man es bei der Dichte und Schallgeschwindigkeit gethan hat, und das Mittel der gefundenen Coëfficienten, für alle Jahr- 
ringe derselben Walze, hat man für jenen einen Jahrring adoptirt; und wenn für alle Jahrringe nur eine Serie von Beobachtungen vorhanden war, so bediente man sich des Mittelwerthes aller Coëfficienten für alle Trumme derselben Holzart. Da das nicht anging, wenn die Beobachtungen von 20 Percent zu sehr entfernt waren, so hat man in diesem Falle in die Tabelle jene Coëfficienten eingetragen, welche mit der dieser Grenze am meisten naheliegenden Feuchtigkeit correspondirten.

Wenn man die Tabelle Nr. XII durchgeht, so wird man sehen, dass die Bewegung der mechanischen Eigenschaften in den verschiedenen Jahrringen ein und desselben Trummes dieselbe ist. So ist der dichteste Jahrring gewöhnlich derjenige, welcher den Schall mit der grössten Geschwindigkeit leitet, welcher folglich auch den grössten Elasticitätscoëfficienten hat und endlich auch der, welcher die bedeutendste Festigkeit aufweist. Nichtsdestoweniger zeigt das Beispiel der Rothbuche Nr. 41 (1), dass die Dichte und Schallgeschwindigkeit manchmal im entgegengesetzten Sinne sich bewegen. Für die Mehrzahl der Bäume, die wir in Stäbe verarbeitet haben, ist die Bewegung der mechanischen Eigenschaften vom Centrum gegen den Umfang genau die entgegengesetzte von jener, welche man bisher allgemein angenommen hat. In der That gehen die Dichte, die Schallgeschwindigkeit, der Elasticitätscoëfficient und die Festigkeit in nahezu constanter Zunahme vom Mittelpunkt gegen den Umfang für Tanne, Fichte, Weissbuche, Esche, Ulme, Ahorn, Sycomore, Zitterpappel und Erle. Es kommt indessen manchmal vor, dass die Ziffern, welche diese Eigenschaften für die der Rinde zunächst gelegenen Stäbe ausdrïcken, wieder ein wenig abnehmen. Besonders bei den harzreichen Bäumen, für welche eine grosse Zahl vergleichender Versuche angestellt worden ist, ist diese constante Zunahme merkwürdig, sie ist derart, dass in den starken Bäumen der Elasticitätscoëfficient der äusseren Jahrringe oft doppelt, manchmal selbst mehr als das doppelte desjenigen vom Kern desselben Baumes beträgt. Die Alkazie folgt im allgemeinen, ohne dieselbe Regelnässigkeit zu zeigen, demselben Gang, wie die obigen Holzarten.

Aber das gleiche gilt nicht mehr für die Eiche und die Birke. In den Bäumen, die wir gepriift haben, findet sich das Maximum der mechanischen Eigenschaften in einer zwischen dem Umfang und dem Centrum in ungefähr einem Drittel des Radius rom Mittelpunkte aus, liegenden Jahrringe. Vom Centrum ausgehend nehmen die mechanischen Eigenschaften bis zu jener 
Schichte zu, um von da ab wieder zu fallen selbst bis unter die Höhe am Ausgangspunkt.

Bei der Rothbuche, von welcher wir mit zwei Exemplaren, einem 50 und einem 95 Jahre alten Baum gearbeitet haben, giengen die Eigenschaften in jedem der beiden anders. Sie nahmen in dem 50 Jahre alten Baume fast regelmässig vom Mittelpunkt gegen den Umfarg hin zu, und in jenem von 95 Jahren, bei welchem uns der Mittelpunktsjahrring fehlte, nahmen sie in derselben Richtung ab. Dieser so bemerkenswerthe Einfluss des Alters, welchen uns die Buche festzustellen gestattete, sollte auch bei der Beurtheilung der Resultate, wolche bei den anderen Holzarten gefunden wurden, in Betracht gezogen werden. Wirklich zählten die beiden Eichen, welche wir untersucht hatten, 95 und 164 Jahre, die Birke 114, die anderen Bäume von 26 bis 61 , ausgenommen eine starke 110 Jahre alte Tanne. Muss man daraus nicht folgern, dass bei den Splintbäumen, das sind jene, deren älteste Jahrringe mit dem Alter absterben (verwachsen), um das, was man Kernholz nennt, zu bilden, wie z. B. die Eiche, Buche etc., ein gewisser Zcitpunkt im Leben cintritt, wo die durch das Alter bedingte Veränderung in dem relativen Zustand ihrer Jahrringe zu einer Umkehr in der Bewegung der mechanischen Eigenschaften führt, wïhrend bei den Holzarten, deren sämmtliche Jahrringe für Flüssigkeiten rlurchdringbar bleiben, wie zum Beispiel die harzreichen Bäume und die meisten weichen Hölzer, diese Eigenschaften vom Mittelpunkte gegen den Umfang hin, bei was immer für einem Alter, zunehmen?

Wir haben in die vorangehenden Betrachtungen nicht die die Pappel betreffenden Resultate cimbezogen, weil der Baum, dessen wir uns bedient haben, am Fusse krank war.

Um mit einem Blick die Bewegung der mechanischen Eigenschaften in den aufeinanderfolgenden Jahringen eines jeden Trummes zu erfassen, kann man die Resultate unserer Versuche graphisch darstellen. Indem man die Jahrringe als Abscissen und ihre Dichten, Schallgुeschwindigkeiten, Elasticititseö̈ficienten oder Festigkeiten als Ordination aufträgt, erhielt man Curren, welche jede dieser Eigenschaften in ihrer Bewegung in dem Trumme oder aber die relative Bewegung einer dieser Eigenschaften in verschiedenen Trummen darstellen.

V. Dic Untersuchung der Veränderungen, wolche die mechanischen Eigenschaften der Höhe des Baumes nach erleider, sei es dass man diese Veränderungen im Sinne der Faser, fuir 
einen einzelnen Jahrring oder für die ganze Masse des Baumes ins Auge fasst, sei es dass man sie in auf die Faser senkrechten Richtungen studirt, diese Untersuchung bildet eine der wichtigsten von jenen Fragen, die wir zu behandeln uns vorgelegt haben. Alle Ergebnisse, welche sich auf diese Frage beziehen, sind in der Tabelle Nr. XVI vereinigt.

Diese Tabelle selbst ist jedoch nur ein Resumé von jenen, die ihr vorangehen, und bevor wir nicht erklärt haben, wie diese entstanden sind, können wir die Besprechung jener nicht beginnen.

Wir haben schon gesehen, dass die Tabelle Nr. XII die Werthe der Dichten, Elasticitätscoëfficienten und Festigkeiten, auf einen Feuchtigkeitsgrad von 20 Percent zurückgeführt, für jeden Jahrring enthielt; aber man musste aus den für jeden Jahrring gefundenen Werthen die Mittelzahl ableiten, die auf das ganze Trumm anwendbar ist, um nicht nur die Methode der Ausdehnung mit jener der Biegung, sondern auch um alle Stammabschnitte eines und desselben Baumes, ob sie nun zerschnitten worden waren, oder nicht, untereinander vergleichen zu können. Zu diesem Behufe haben wir die Radien der Trumme und die Breite der aufeinander folgenden Ringe gemessen (siehe Tabelle Nr. XIII), aus denen die Stäbe genommen wurden, und unter der Annahme, dass die Eigenschaften jedes Stabes sich in allen benachbarten, denselben Ring zusammensetzenden Jahrringen auch vorfinden, hatten wir nur die Dichte, den Elasticitätscoëfficienten und die Festigkeit jedes Ringes mit dessen Flächeninhalt zu multipliciren, diese Producte zu summiren und durch den Querschnitt der ganzen Walze jene Summe zu dividiren. Auf diese Art fanden wir die gesuchten Mittelzahlen, welche wir in die drei letzten Columnen der Tabelle Nr. XII eingetragen haben. Wir haben eine ähnliche Rechnung für die Schallgeschwindigkeiten nicht anstellen können, weil es ein neuerliches Problem gewesen wäre, die Schallgeschwindigkeit für einen Cylinder, der aus concentrischen Röhren, von denen jede mit einer verschiedenen derartigen Geschwindigkeit begabt ist, zu bestimmen.

Die Tabelle Nr. XIV bringt die Daten und die Ergebnisse aller bei der Ausbiegung der Walzen unternommenen Versuche, die durch die Versuche festgestellten Dichten und Elasticitätscoëffiicienten, die Feuchtigkeitsgrade der Trumme und die mittelst der vorangehend bestimmten Coëfficienten auf 20 Percent Feuchtigkeit zurückgefülırten Werthe. Unter diesen Trummen gibt es mehrere, die von demselben Baume zu verschiedener Höhe herstammen und die folglich in die Tabelle Nr. XVI aufaunehmen waren. 
Die Tabelle Nr. XV enthält febenso die Daten und die Resultate der Versuche, die mit den senkrecht auf die Fasern im Sinne des Radius und der Tangente der Jahrringe, in der Höhe der verschiedenen Trumme entnommenen Barren mittelst transversaler Schwingungen und über die Zugsifestigkeit durchgeführt wurden.

Endlich in der Tabelle Nr. XVI findet man:

(a) Die Werthe der mechanischen Eigenschaften im Sinne der Fascr für eine und dieselbe Holzschichte bei verschiedenen IIöhen. Um diese Untersuchung machen zu können, haben wir die Stäbe in den gleichen Jahrringen der verschiedenen von ein und demselben Baume herrührenden Trumme genommen, was sehr leicht durchführbar ist, indem man nur vom Umfang aus die Jahrringe bei der Zertheilung abzählt. Diese correspondirenden Stäbe sind nicht durch die in den vorangehenden Tabellen erscheinenden Ziffern, sondern durch die Buchstaben $a, b, c, d$ bezeichnet, wobei mit $a$ der der Rinde zunächst gelegene Stab benannt wurde.

(b) Die mechanischen Eigenschaftsn der ganzen Trumme, die entweder direkt durch Ausbiegung gewonnen, oder aus den Ausdehnungsversuchen mit den Stäben abgeleitet wurden. Die Walzen wurden nach ihrer Höhe im Baume, vom Fusse ausgehend, geordnet.

(c) Die mechanischen Eigenschaften im Sinne des Radius bei verschiedenen Höhen.

(d) Dieselben im Sinne der Tangente an die Jahrringe.

Die in dieser Tabelle enthaltenen Ziffern zeigen, dass im allgemeinen die mechanischen Eigenschaften mit der Höhe im Baume für alle im Sinne der Faser abgesondert geprüften Jahrringe an Grösse abnehmen. Diese Abnahme ist beträchtlich genug und findet sich gleichfalls in den auf die Axe des Baumes senkrecht nach den beiden Richtunger genommenen Barren wieder; aber wenn man die ganzen in verschiedenen Höhen aus dem Baume geschnittenen Walzen untereinander vergleicht, so gewahrt man nicht mehr diese regelmässige Bewegung der Eigenschaften, was sich übrigens a priori hatte vorausschen lassen. Wir haben ja bei gewissen Bäumen gesehen, dass die mechanischen Eigenschaften am schwächsten in den äusseren Jahrringen auftreten; andererseits gehen sie gleichmissig abnehmend in jedem Jahrring von dem Fuss gegen den Gipfel hin: und da es nur die äusseren Holzschichten sind, die sich bis in die Hölie fortsetzen, so ist es einleuchtend, dass die obersten Theile des Stam- 
mes, welche von jenen in ihrer Gesammtheit gebildet sind, bei diesen Bäumen mit minder bedeutenden Ziffern der Eigenschaften, als die Theile am Fusse auftreten. Das ist unter anderem bei der Eiche Nr. 34 der Fall. Aber es findet diess bei jenen Bäumen nicht statt, deren äussere Schichten die besseren sind. Bei diesen wird das Verhältniss zwischen den Werthen der Eigenschaften jener Trumme, die an der Basis und jenen, die vom Wipfel gewonnen wurden, von der Beziehung des Gesetzes der Zunahme der Eigenschaften der verschiedenen Jahringe vom Centrum gegen den Umfang, zu dem Gesetze der Abnahme in ein und demselben Jahrring von der Wurzel gegen das Zopfende hin abhängen. Es kann demnach bei diesen Bäumen Vergrösserung, Gleichförmigkeit und Verringerung der Höhe der Eigenschaften vorkommen, und es verhält sich demgemäss auch in Wirklichkeit so. Indessen sind die zahlreichsten Fälle bei den von uns angestellten Versuchen doch jene gewesen, in denen die Eigenschaften von unten gegen oben hin abnehmen.

VI. Wir haben nun zu zeigen, wie der Elasticitätscoëfficient nicht nur im Sinne der Faser, sondern auch nach dem Radius und der Tangente mit der Höhe im Baume variirt. Selbstverständlich sind wir weit davon entfernt, diese Linien als wirkliche Axen der Elasticität anzusehen; denn damit eine solche Auffassung berechtigt wäre, müsste nicht blos die Elasticität der ganzen Länge der Linien nach dieselbe bleiben, sondern auch in allen Parallelen gleich gross sein. Beim Holze findet indessen nichts ähnliches statt. Die Elasticität variirt mit der Höhe, sie variirt vou Jahrring zu Jahrring, endlich in ein und demselben Durchmesser mit der Entfernung des Barrens vom Mittelpunkt, - diess zeigt das Beispiel des Barrens $R$ vom Fusse der Fichte Nr. 31 (siehe Tabelle Nr. XV). Dieser Barren wurde zuerst als ganzes gepriift, hierauf in zwei gleiche Theile geschnitten, von denen der eine a vom Mittelpunkt bis zum Halbirungspunkt des Radius, und der andere $b$ von diesem bis zum Umfang ging. Der Elasticitaitscoëfficient dieses letzteren Theiles wurde höher gefunden als jener der ersteren, was sehr gut mit dem Gang der Elasticitit in den Jahrringen dieses Baumes ïbereinstimmt. Natiirlich wird man auch Differenzen derselben begegnen, wenn man einen von der Peripherie mehr oder minder entfernten Barren vornimmt. Wir haben nun die Verhältnisse der Elasticitäten und Festigkeiten in dieser dreifachen Richtung, wegen der praktischen Wichtigkeit dieser Daten, untersucht. 
Diese Verhältnisse (Tabelle Nr. XVI) bleiben in demselben Baume, bei verschiedenen Hïhen, merklich die grleichen, aber sie variiren mit den Holzarten. Wenn man die Elasticitäten und Festigkeiten im Sinne der Fasern als Einheiten annimmt, so findet man als allgemeine Mittelzahlen für die Elasticitäten im Sinne des Radius und der Tangente die Zahlen 0.165 und 0.091 und für die Festigkeiten nach diesen beiden Richtungen 0.163 und 0.159.

VII bis X. Wir haben schon, als wir von der Wahl der Bïume gesprochen haben, gesagt, dass wir verschiedene Stämme von Eichen, Rothbuchen, Tannen und Fichten genommen haben, mittelst deren wir hofften, den Einfluss, den das Alter, die Stärke der Jahrringe, die Exposition, die Bodenbeschaffenheit und die Fällungszeit auf die mechanischen Eigenschaften üben, zu erkennen.

Bei der Discussion der Methoden haben wir die Scbwierigkeiten, die sich bei Anwendung der bekannten Formeln auf die Pfeilhöhen der Ausbiegungen harzreicher Hölzer ergeben haben, und welche uns auch nicht erlaubten Schliusse zu ziehen, hervorgehoben, aber wir haben wenigstens zum Theil die Serie der tannenen Walzen durch die der Praxis üblichen viercekig behauenen Stiicke aus dieser Substanz ersetzen können und auf diese Art einige Vergleichs-Resultate für die harzreichen Hölzer erhalten.

Die 'Tabelle Nr. XIX enthält:

1. Die für die Dichten- und Elasticitätscoëfficierten der Trumme von Eichen und Rothbuchen auf 20 Percent Feuchtigkeit zurückgeführten Werthe. Diese Ziffern sind nach dem Alter und der Herkunft der Bäume etc. geordnet, um die Discussion zu erleichtern.

2. Die ebenso nach Herkunft und Grösse der viereckig behauenen Stücke der 'Tanne geordneten Werthe der Dichten, Elasticitïtseö̈fieienten und Festigkeiten. Es fehlten uns dabei zwei Daten: Das Aiter der Bäume und der Feuchtigkeitsgrad, welcher für diese Stïcke nicht erhoben wurde. Man kann indessen annehmen, dass das Alter zicmlich der Stärke proportionel gewesen ist und dass der Fenchtigkeitsgrad sich jenem von 20 Perzent näherte, da die Bäume ein Jahr gefällt waren.

Diese Tabelle laisst erkennen, dass die Dichte mindestens innerhalb der Grenzen unserer Versuche vollkommen unabhängig von den Einflüssen gewesen sei, die wir zu erforschen suchten, und dass ihre Abweichungen für Bäume ein und derselben Art wenig betrïchtlich seien. Sie zeigt nur bei der Tanne etwas grössere Abweichungen, was dem Umstand, dass ihre Wcrthe 
nicht auf 20 Perzent Feuchtigkeit reducirt werden konnten, zugeschrieben werden muss.

Dasselbe ist aber nicht bei dem Elasticitätscoëfficienten der Fall, welcher in dem Maasse als das Alter zunimmt, abzunehmen scheint. Diese Abnahme ist bei den unter vergleichbaren Umständen genommenen Eichen constant gewesen. Sie hat auch bei den Tannen und Rothbuchen, wiewohl für letztere in einer weniger erwiesenen Weise, stattgefunden.

Die grössere oder geringere Trockenheit und die Exposition des Bodens, auf denen die Bäume erwachsen sind, scheinen ebenfalls auf den Werth des Elasticitätscoëfficienten zu reagiren.

So haben die in nördlichen, nordwestlichen und nordöstlichen Lagen und auf trockenem Grunde erwachsenen Bäume immer einen grösseren und einen um so grösseren Coëfficienten, wenn diese beiden Umstände vereinigt auftreten; ebenso wie die auf sumpfigen Boden entstandenen Bäume mit wenig Ausnahmen die kleinsten Coëfficienten zeigen. Bei der Rothbuche ist besonders die Wirkung dieser Umstände eine markirte.

Das Alter und die Exposition influenciren auf die Festigkeit und Elasticität der Stücke von Tannenholz im gleichen Sinne, was zeigt, dass der Gang der Festigkeit mit jenem der Elasticität im Einklange steht.

Die Elasticitätscoëfficienten der am Vogesensandstein erwachsenen Rothbuchen sind alle viel grösser bei den vergleichbaren Bäumen als jene der auf rem bunten Sandstein oder auf dem Muschelkalk gediehenen Rothbuchen. Wir haben ein ähnliches Gesetz bei der Eiche nicht gefunden. Was die Tanne anbelangt, so erinnern wir daran; dass wir nur ein vom Vogesensandstein gekommenes Exemplar zu unserer Verfügung hatten. Es wäre schwierig, aus dem cinzelnen Beispiel der Rothbuche eine allgemein giltige Folgerung zu ziehen. Da man gesehen hat, wie sehr die Trockenheit des Bodens auf die Elasticität dieses Baumes influencirt, so wäre es wohl möglich, dass die grosse Durchdringlichkeit der oberen Schichten des Vogesensandsteines, welche das Regenwasser nicht lange Zeit zurickzuhalten gestattet, theilweise an der grösseren Elasticität der auf einem Boden von dieser Formation erwachsenen Rothbuchen Schuld sei.

Die Bäume, welche zur vollen Saftzeit gefällt und von denen die einen entrindet und der Sonne ausgesetzt, die andern nicht entrindet und unmittelbar in den Hangar transportirt wur- 
den, wo auch die ausser der Saftzeit gewonnenen Trumme aufbewahrt waren, haben keinerlei Eigenthümlichkeiten gezeigt. Aber da wir nur eine kleine Zahl von Bäumen unter diesen Bedingungen vorgenommen hatten, so bedürfte es neuerlicher specieller und mehr zahlreicher Untersuchungen, um den Einfluss der Fällung während der Saftzeit zu bestimmen. Nichtsdestoweniger kïnnen wir aus den unsrigen schliessen, dass der Elasticitätscoëfficient der Hölzer durch die Fällungszeit nicht merklich modificirt wird. Was ihre Aufbewahrung anbelangt, so ist es cbenfalls nicht wahrscheinlich, dass sie einen Einfluss nehme, übrigens liegt diese Frage vollständig ausserhalb derjenigen, welche wir in dieser Arbeit zu studieren hatten.

Wir hatten endlich den Einfluss der Breite der Jahrringe auf die mechanischen Eigenschaften der Hölzer zu untersuchen. Wir haben dies in der Art gemacht, dass wir die mittlere Jahrriugbreite für jedes Trumm bestimmten, und haben gefunden, dass sie in einer von der Bewegung der Eigenschaften vollkommen unabhängigen Weise variirte. Aber da diess möglicher Weise der Abwechslung von schmalen und breiteren Jahrringen, welche fast in allen Bäumen auftritt, zugeschrieben werden muss, so haben wir untersucht, was für die Stäbe, welche nur einige wenige gleichbreite Jahrringe enthalten, der Fall sei. Die Tanne hat uns allein eine Relation zwischen der Breite der Jahrringe und ihrer Elasticität gezeigt. Diese letztere ist um ebensoviel grösser, als die Jahrringe kleiner werden. Indessen ist die Elasticität auch bei gleichbreiten Jahrringen verschieden und zwar mit Rücksicht auf die Entfernung derselben vom Centrum, sie ist immer grösser in jenen, die vom Centrum mehr entfernt sind. Die Abnahme der Jahrringbreite ist daher in der Tanne, wenn man vom Centrum gegen den Umfang vorschreitet, nicht die erste Ursache der Zunahme der Elasticität, die man dabei findet. Nach dem Vorangehenden hat man Ursache zu glauben: dass die vorzügliche Beschaffenheit der nordischen Fichten nicht der geringen Breite ihrer Jahrringe ausschliesslich zuzuschreiben sei, sondern dass sie vielmehr von Klima und Boden herrührt, in dem sie erwachsen sind.

XI. Wir haben schon bemerklich gemacht, dass in einem und demselben Baume bei demselben Feuchtigkeitsgrade die Eigenschaften im Allgemeinen sich in gleichem Sinne bewegen, so dass derjenige Jahrring, welcher in einer grossen Höhe die grösste Dichte zeigt, auch mit der grössten Elasticität und Festigkeit begabt ist. Dieses mit den Stäben gewonnene Ergebniss 
wurde namentlich bezüglich des Verhältnisses der Dichte zur Festigkeit durch spezielle, mit sechs Tannen angestellte Versuche, von denen drei in Bretter und die andern in Bohlen geschnitten worden waren, bestätiget.

Die Fig. 1-6 der Taf. II zeigen die bezüglichen Stellungen dieser Stücke in den Bäumen, ihre Dimensionen, ihre absoluten Gewichte $\pi$ und die Belastungen $\mathrm{P}$, welche den Bruch nach der oben beschriebenen Methode und mit dem für Stücke dieser Art dienenden Apparat herbeigeführt haben.

Das Verhältniss der verschiedenen mechanischen Eigenschaften untereinander war zu unregelmässig und zu wenig constant, selbst in ein und demselben Baume, um durch eine Formel ausgedrückt werden zu können.

Wenn man die Bäume derselben Holzart untereinander verglichen hatte, so wiederholte sich nur die Beziehung zwischen Elastizität und Festigkeit, sie verschwand jedoch vollständig, sobald man diesen Vergleich unter den für verschiedene Holzarten gefundenen Mittelzahlen anstellte. Es ist wohl richtig, dass die Fichte und die Pappel, deren Dichte eine sehr geringe ist, auch die kleinsten Elasticitätscoëfficienten, Elasticitätsgrenzen und Festigkeiten zeigen, aber andererseits hat die Tanne, welche ebenfalls von sehr geringer Dichte ist, einen schr grossen Elasticitätscoëfficienten; die Weissbuche und die Rothbuche, welche ein selur hohes spec. Gewicht haben, zeigen nur eine geringe Festigkeit, und endlich die Akazic, welche allen Holzarten beziiglich der Elasticität und der Festigkeit überlegen ist, hat eine Dichte, welche derjenigen von mehreren Bäumen nachsteht.

XII. Bevor wir die aus allen unseren Versuchen hervorgehenden Mittelzahlen angeben, welche wir zur praktischen Anwendung am geeignetsten halten, müssen wir auf die Elasticitätsgrenze und auf die permanente Maximalausdehnung zurïckkommen, von welcher wir bisher nur gesprochen haben, als wir andeuteten, dass es unmöglich sei, für dieselben präcise Werthe zu bestimmen, und dass die Ergebnisse je nach der Genauigkeit der angewendeten Instrumente und der Art zu operiren von einander abweichen müssen.

Wir haben für den Ausdruck der Elasticititsgrenze das Gewicht in Kilogrammen per Quadr.-Millimeter Querschnitt genommen, welches, nachdem es eine genug kurze Zeit eingewirkt, eine permanente Verlängerung durch Zug ron 0.00005 der Länge hervorgebracht hat. Wir haben diese Grenze gewiihlt, damit unsere Ziffern mit jenen anderer Autoren rergleichbar sein konnten. 
Man hätte sicherlich viel niedrigere Grenzen gefunden, wenn man schon bei den ersten messbaren permanenten Ausdehnungen stehen geblieben wäre.

Die folgenden Ziffern machen den Einfluss des Feuchtigkeitsgrades auf den Werth dieser Gränze ersichtlich.

\section{Elasticitiits-Grenzen.}

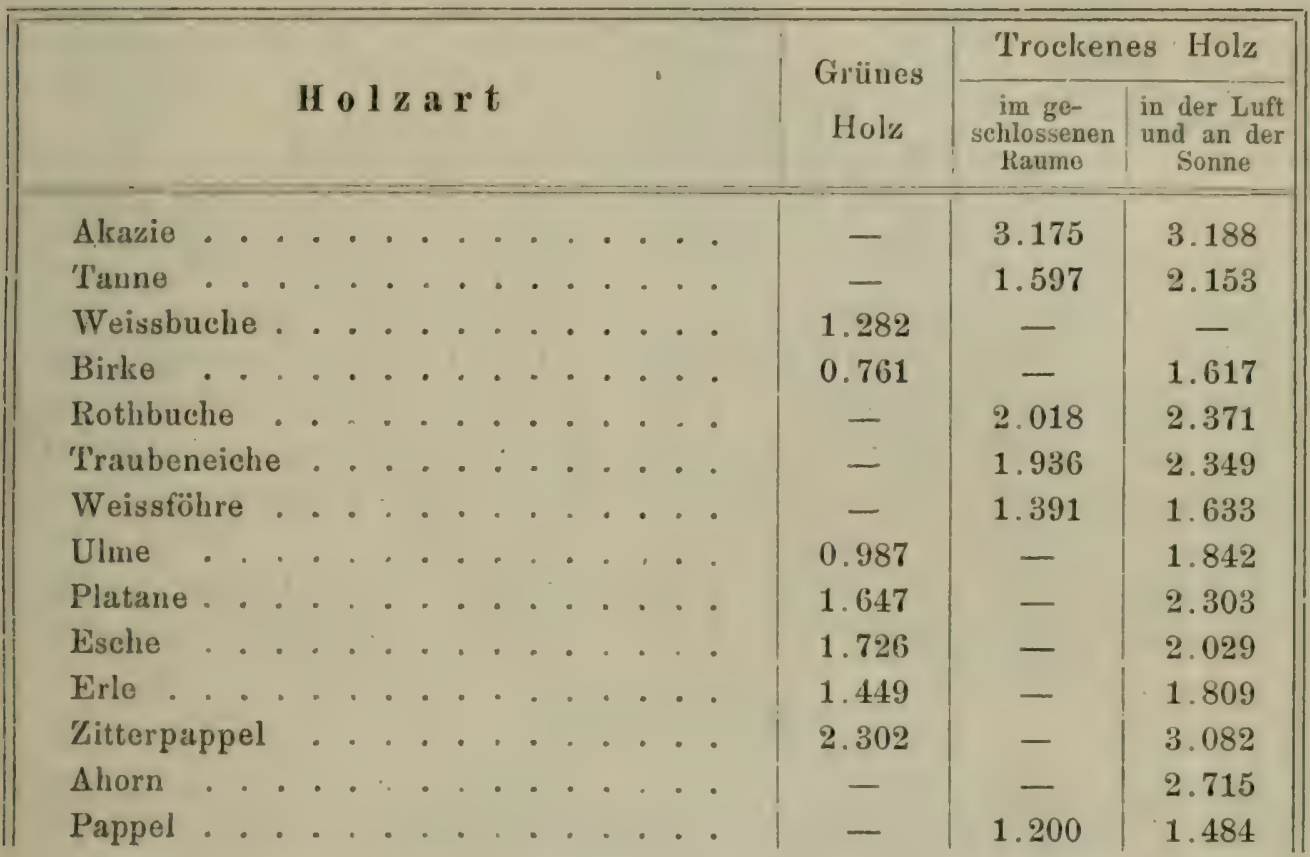

Man sicht, dass dieser Werth mit der Trockenheit zunimmt, woraus sich erklärt, warum die sehr nassen Hölzer viel leichter als die trockenen permanente Krümmungen annehmen.

Boi den, im Ofen stark getrockneten Hölzern, fällt die Elasticitïtsgrenze fast mit jener Belastung zusammen, welche den Bruch hervorbringt, d. h. die Hölzer vertragen fast keine permanente Ausdehnung in diesem Falle.

Die Maximalausdehnung besteht aus zwei Theilen; der eine, die elastische Ausdehnung, ist immer sehr leicht durch den Elasticitätscoëfficienten nach der Belastung, welche den Bruch hervorruft zu berechnen. Den andern Theil bildet die permanente Ausdehnung, welche für die Hölzer sehr klein ist. Wir haben gefunden, dass diese letztere für das grrine Holz höchstens 0.0007 und für das getrocknete 0.00032 der Lïnge beträgt. Es wäre demnach iiberflüssig, sie einzeln für jede Holzart anzugeben, man hat blos zu beachten, dass sie mit der Trockenheit abnimmt, d. h. je trockener die Hölzer sind, desto weniger lassen sie sich ausdehnen. 
Es folgen nun hier die aus unserer Arbeit hervorgehenden praktischen Daten.

\section{Mittelzahlen,}

welche aus den Versuchen mit mehreren Bäumen derselben Holzart im Sinne der Faser angestellt wurden, auf 20\% Feuchtigkeit reducirt.

\begin{tabular}{|c|c|c|c|c|c|c|c|c|c|}
\hline \multirow{3}{*}{ Holzart } & \multirow[b]{3}{*}{ 总 } & \multirow{3}{*}{ 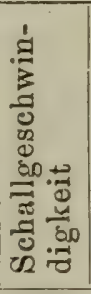 } & \multirow{3}{*}{ 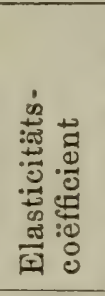 } & \multirow{3}{*}{\begin{tabular}{|c|} 
Verhältniss \\
zwischen dem \\
aus Vibratio- \\
nen abgeleite- \\
ten und dem \\
durch Ausdeh- \\
nung gefunde- \\
nen Elastici- \\
tätscoëficien- \\
ten \\
\end{tabular}} & \multirow{3}{*}{ 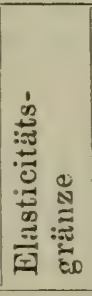 } & \multirow{3}{*}{ 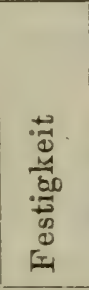 } & \multicolumn{3}{|c|}{$\begin{array}{l}\text { Für einen Verlust von } \\
1 \% \text { Fenchtigkeit }\end{array}$} \\
\hline & & & & & & & \multirow[b]{2}{*}{ 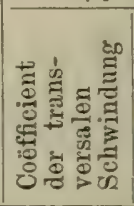 } & \multicolumn{2}{|c|}{$\begin{array}{c}\text { Variationscoëffi- } \\
\text { cient }\end{array}$} \\
\hline & & & & & & & & 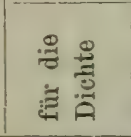 & 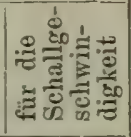 \\
\hline Akazie... & 0.717 & 14.19 & 1261.9 & 1.193 & 3.188 & 7.93 & 0.00300 & 0.00555 & 0.00576 \\
\hline Tanne... & 0.493 & 13.96 & 1113.2 & 1.056 & 2.153 & $4,18 \%$ & 0.00467 & 0.01034 & 0.00797 \\
\hline Weissbuche & 0.756 & 11.80 & 1085.7 & 1.105 & 1.282 & 2.99 & 0.00149 & 0.00743 & 0.00951 \\
\hline Birke . . & 0.812 & 13.82 & 997.2 & 1.212 & 1.617 & 4.30 & 0.00347 & 0.00422 & 0.00943 \\
\hline Rothbuche . & 0.823 & 10.06 & 980.4 & 1.087 & 2.317 & 3.57 & 0.00412 & 0.00486 & 0.01068 \\
\hline Stieleiche & 0.808 & - & 977.8 & 一 & - & 6.49 & - & - & - \\
\hline Traubeneiche & 0.872 & 11.58 & 921.3 & 1.117 & 2.349 & 5.66 & 0.00461 & 0.00420 & 0.00805 \\
\hline Weissföhre & 0.559 & 10.00 & 564.1 & 1.086 & 1.633 & 2.48 & 0.01093 & 0.01056 & 0.01369 \\
\hline
\end{tabular}

\section{Mittelzahlen,}

welche aus Versuchen mit einem einzigen Baume von jeder Holzart im Sinne der Faser hervorgingen, auf 20\% Feuchtigkeit reducirt.

\begin{tabular}{|c|c|c|c|c|c|}
\hline Ulme & \begin{tabular}{|l|l|l|}
0.723 & 12.40 \\
\end{tabular} & 1165.3 & 1.175 & 1.8426 .99 & 0.002940 .003860 .01006 \\
\hline Platane & 0.69213 .43 & 1163.8 & 1.139 & 2.3036 .16 & 0.003120 .004230 .00540 \\
\hline Esche & $0.697: 14.05$ & 1121.4 & 1.246 & $2.029,6.78$ & $0.00121,0.005010 .00489$ \\
\hline Erle . . & 0.60113 .95 & 1108.1 & 1.121 & 1.8094 .54 & 0.002800 .001100 .00897 \\
\hline Zitterpappel & 0.60215 .30 & 1075.9 & 1.035 & 3.0827 .20 & $0.003850 .00230 \quad 0.00803$ \\
\hline Ahorn . . & 0.67412 .36 & 1021.4 & 1.068 & $2.715 \mid 3.58$ & $0.00328 \quad 0.003630 .00929$ \\
\hline Pappel . • & $0.477 \quad 12.89$ & 517.2 & 1.007 & $1.48 \pm 1.97$ & $0.00583 \quad 0.00450,0.00592$ \\
\hline
\end{tabular}

Anmerkung. Die Schallgeschwindigkeiten sind Mittelzahlen der direct aus den Stäben gefundenen Geschwindigkeiten während die Elasticitätscoëfficienten Mittelzahlen aus der Gesammtheit unserer Versuchsergebnisse sind, die beiden stellen daher keine correspondirenden Zahlen dar.

* Die Ziffer 4.18 für die Festigkeit der Tanne begreift die Ergebnisse specieller Versuche mit Bohlen oder Brettern nicht in sich; weun man auf diese die Approximativ-Formel

$$
R=\left(P+\frac{\pi}{2}\right) \frac{3 \mathrm{a}}{\mathrm{bc}^{2}}
$$

anwendet, so erhält man im Mittel für die Festigkeit der Bohlen 5.88 und für jene der Bretter 6.50. 


\section{Versuche}

im Sinne des Radius und der 'Tangente angestellt.

\begin{tabular}{|c|c|c|c|c|c|c|}
\hline \multirow[b]{2}{*}{ Holzart } & \multicolumn{3}{|c|}{ In Sinne des Rudius } & \multicolumn{3}{|c|}{ Im Sinne der 'Tangente } \\
\hline & $\begin{array}{l}\text { Elasticitat- } \\
\text { coefficient }\end{array}$ & $\begin{array}{l}\text { Schall- } \\
\text { geschwin- } \\
\text { digknit }\end{array}$ & Festigkeit & $\begin{array}{c}\text { Elasticitäts- } \\
\text { couffficient }\end{array}$ & $\begin{array}{l}\text { Schall- } \\
\text { geschwin- } \\
\text { digkeit }\end{array}$ & Festigkeit \\
\hline W eissbuche & 208.4 & 5.14 & 1.007 & 103.4 & 3.60 & 0.608 \\
\hline Zitterpappel & 107.6 & 4.86 & 0.171 & 43.7 & 2.74 & 0.414 \\
\hline Erle . . & 98.3 & 412 & 0.329 & 59.4 & 3.14 & 0.175 \\
\hline Platane. . & 134.9 & 4.51 & 0.522 & 805 & 3.42 & 0.610 \\
\hline Ahorn . . & 157.1 & 4.63 & 0.716 & 72.7 & 3.12 & 0.371 \\
\hline Eiche. . . & 188.7 & 4.62 & 0.582 & 129.8 & 3.88 & 0.406 \\
\hline Birke . . . & 81.1 & 3.23 & 0.823 & 155.2 & 4.57 & 1.063 \\
\hline Rothbuche . & 269.7 & 5.53 & 0.885 & 159.3 & 4.26 & 0.752 \\
\hline Esche ... & 111.3 & 4.19 & 0.218 & 102.0 & 3.80 & 0.408 \\
\hline Ulıne... & 122.6 & 4.28 & 0.345 & 63.4 & 3.05 & 0.366 \\
\hline Pappel . . & 73.3 & 4.22 & 0.146 & 38.9 & 3.16 & 0.214 \\
\hline Akazie : . & 170.3 & 4.44 & - & 152.2 & 4.07 & 1.231 \\
\hline Tanne . . & 94.5 & 4.02 & 0.220 & 34.1 & 3.26 & 0.297 \\
\hline Weissföhre . & 97.7 & 4.23 & 0.256 & 28.6 & 2.39 & 0.196 \\
\hline
\end{tabular}

\section{Versuch e}

mit Balken, Bohlen und Brettern von Tannenholz.

\begin{tabular}{|c|c|c|c|c|c|c|c|c|c|}
\hline $\begin{array}{l}\text { Uebliche } \\
\text { Bezeichnung }\end{array}$ & 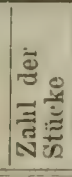 & 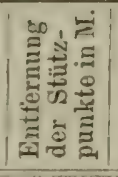 & 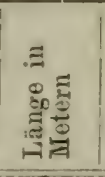 & 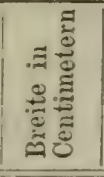 & 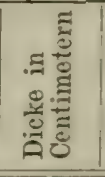 & 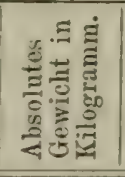 & 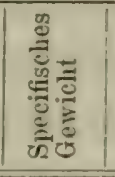 & 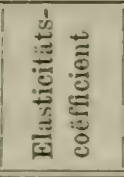 & 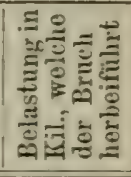 \\
\hline $11 / 12 \cdot$. & 1 & 0 & 14.00 & 28.99 & 41 & 697 & 0 & 113 & $6 \pm 0$ \\
\hline $3 / 10 \ldots . .$. & 1 & 11.00 & 13. & 25.46 & & 475 & 6 & 115 & 5394 \\
\hline$s / 9 \cdot \ldots \cdot \cdots$ & 3 & 9.00 & 10.48 & 22.30 & 24.30 & 310. & 0.548 & 1026.9 & 3447 \\
\hline $6 / 7 \ldots \ldots$ & 3 & 9.00 & 10.46 & 16.99 & 19.63 & 183. & 0.525 & 1245.0 & 2082 \\
\hline Sparren (Cherrón) . & 3 & 9.00 & 10.47 & 9.27 & 12.31 & 57.48 & 0.481 & 1257.6 & 517 \\
\hline Bohle (Uadrier) . . & 15 & 3.02 & 4.24 & 24.63 & 5.40 & 27.79 & 0.493 & 1089.8 & 917 \\
\hline Brett (Planche) & 42 & 3.02 & 4.25 & 24.13 & 2.78 & 13.74 & 0.479 & 1202.2 & 264 \\
\hline
\end{tabular}

\section{Versuch e}

mit Balken, Bohlen und Brettern aus Eichenholz.

\begin{tabular}{|c|c|c|c|c|c|c|c|c|}
\hline $8 \frac{1}{2} / 9 \frac{1}{2} \cdot \ldots .$. & 1 & 5.50 & 5.87 & 23.18 & 25.28 & 346.701 .008 & 825.1 & 7889 \\
\hline $8 / 9 . . .$. & 1 & 5.50 & 6.11 & 21.67 & 23.67 & 300.300 .958 & $8: 2.3$ & 7189 \\
\hline $7 / 8 \cdot \ldots \cdot \cdot \cdot$ & 1 & 5.50 & 7.06 & 19.07 & 22.00 & $|273.200 .922|$ & 855.9 & 5225 \\
\hline $6 / 7 \ldots . .$. & $\ell$ & 5.50 & 6.82 & 15.99 & 18.90 & $191.30,0.928$ & 1007.0 & $5525)$ \\
\hline $5 / 6 \cdot$. & 1 & 5.50 & 6.54 & 13.67 & 16.10 & $141.70,0.958$ & 638.1 & $2220)=$ \\
\hline Sparren (Cherron) . & 1 & 3.00 & $4: 01$ & 8.28 & 8.14 & 17.200 .636 & 601.3 & 540 \\
\hline$"$ & 1 & 2.50 & 4.00 & 7.82 & 8.04 & $19.10 \mid 0.759$ & 774.3 & $735 \frac{0}{0}$ \\
\hline Doppelbrett (Doublette) & 1 & 5.50 & 6.50 & 29.34 & 5.46 & 71.300 .685 & 965.8 & 435 \\
\hline Pfosten (Lichantillou) & 1 & 3.00 & 3.65 & 14.34 & 4.22 & 18.200 .824 & 1210.7 & 375 \\
\hline Schalbrett (Entrerous). & 1 & 3.00 & 3.37 & 24.22 & 2.82 & 16.400 .712 & 1251.2 & $335{ }^{22}$ \\
\hline
\end{tabular}


Es ist nicht immer möglich, von vorne herein aus den Ziffern, die wir geben, zu schliessen, welche die beste Anwendung der Hölzer in der Praxis sei. In der That, hängt die Anwendung der Hölzer sehr oft von ihrer Widerstandsfähigkeit gegen Druck und Torsion von ihrer Härte, von ihrer Structur, von ihrer Unbiegsamkeit und namentlich von ihrer Dauerhaftigkeit ab. Ausserdem müssen wir daran erinnern, dass streng genommen die Ergebnisse unserer Versuche nur auf Hölzer, die aus den Vogesen kommen, anwendbar sind, da ja eben unsere Versuche nur mit solchen durehgeführt wurden.

Die Akazie ist diejenige Holzart, welche in jeder Beziehung mit den vorzüglichsten Eigenschaften begabt ist, sie vereinigt in sich die grösste Festigkeit, den höchsten Elasticitätscoëfficienten und die höchste Elasticitätsgränze mit einer grossen Härte und Dauerhaftigkeit. Dieses Holz von einer bis jetzt sehr beschränkten Verwendung könnte demnach in vielen Fällen sehr’ nützlich verwendet werden und würde ein kostbares Material für EisenbahnSleeper besonders mit Rücksicht auf dessen rapides Wachsthum und dessen leichtem Gedeihen in der Mehrzahl der Bodenarten sein. Sie könnte auf Böschungen und den ausgedehnten Grundcomplexen der Eisenbahnın, also, an denjenigen Orten, an denen sie später verwerthet wird, selbst cultivirt werden.

Die Tanne reiht sich in doppelter Beziehung an die Akazie an, einerseits was die Grösse des Elasticitätscoëfficienten, andererseits die ihrer Festigkeit anbelangt. Wiewohl diese beiden Eigenschaften geringer sind als bei mehreren anderen Holzarten, sind sie doch hinlänglich gross, damit die Anwendung der Tanne in allen Fällen, wo es sich um einen grossen Elasticitätswiderstand, bei relativ geringem Gewichte des Holzstückes handelt, eine sehr vortheilhafte würde. Die geringe Elasticität und Festigkeit im Sinne des Radius und der Tangente machen die Tanne für transversale Inanspruchnahme minder geeignet. Da endlich die stärksten Jahrringe dieser Bäume in der Nähe des Uinfanges gelegen sind, so sollte man sie, anstatt sie viereckig zu behauen, wie es gewöhnlich geschieht, so viel wie möglich in ihrer natürlichen Form zur Anwendung bringen. Aus demselben Grunde sind die, aus der Nähe des Umíanges genommenen Bretter und Bohlen von Tannenholz die besten, und wenn man in der Mitte durchschnittene Stiicke zu verwenden hat, so wird es vortheilhaft sein, sie in der Art zu placiren, dass die grösste Spannung auf den äusseren Theil wirksam ist. 
Die Eiche hat die Eigenthiimlichkeit, dass sie, ohne für irgend eine der mech. Eigenschaften die höchste Ziffer zu geben, alle Eigenschaften in einer sehr bedeutenden Höhe vereinigt. Diese Gesammtheit der Eigenschaften macht sie gleichfalls fast fuir alle Anwendungen geeignet und erklärt die bedeutende Rolle, die sie in der Praxis spielt. Den Verzug, welchen man dem Kernholz gegeniiber dern Splintholz zuschreibt, und dem Holze vom Fusse des Baumes gegenuiber der Krone, ist für die Eiche vollkommen gerechtfertiget, weil in den ersteren Theilen die mechanischen Eigenschaften ihr Maxinium erreichen. Im Einklange mit unseren Versuchen haben wir noch hinzuzufügen, dass das Holz, welches von einem jungen Baume herrührt, fester ist als jenes von einem älteren Baume von gleicher Dicke, endlich dass die Traubeneiche von der Stieleiche in ihren Eigenschaften übertroffen wird.

Die Weissbuche, Rothbuche und Birke haben entweder gleiche oder etwas höhere Elasticitätscoëfficienten als die beiden Varictaten der Eiche, aber ihre Festigkeit ist viel geringer und das gleiche gilt von ihren Elasticitätsgrenzen, ausgenommen die Rothbuche. Diese Bäume sind besonders wegen ihrer grossen Elasticität und Festigkeit in den beiden auf die Faser senkrechten Richtungen beachtenswerth. In dieser Beziehung könnten sie von sehr guter Anwendung beim Eisenbahnbaue sein, vorausgesetzt, dass man im Stande ist, sie ohne Alterirung ihrer mechanischen Eigenschaften zu conserviren. Diese Stärke in den Transversalrichtungen ist auch der Grund, warum sie so gute Zahmradkïmme liefern und die Reihenfolge, in welcher die Praktiker sie in dieser Hinsicht rangiren, stimmt mit den Werthen ihrer Festigkeiten im Sinne des Radius uiberein.

Die Weissföhre gibt uns viel niedrigere Ziffern, als alle andern Holzarten, mit Ausnahme der Pappel. Dieser Umstand hat uns desshalb sehr überrascht, weil man die Elasticitiit der Fohre im allgemeinen jener der Tanne als ebenbürtig und manchmal sogar als derselben iberlegen annimmt. Aber bevor man die Ziffern als den wahren Auschuck der mechanischen Eigenschaften der Vogesen-Föhren ansieht, dürfte es nothwendig sein, neuerliche Versuche mit Bäumen dieser Holzart, welche im geschlossenen Stande erwachsen sind, anzustellen. Wir haben nämlich in den Wäldern, aus denen wir unsere Bäume bezogen haben, gefunden, dass bei den isolirt stehenden Individuen das Wachsthum sehr stark gewesen ist und die Forstverwaltung glaubte nicht, uns einen Baum aus den schönen hochstämmigeen 
Wäldern unserer Umgebung gewähren zu sollen, was uns in die Lage versetzt hätte, diese vergleichenden Versuche anzustellen.

Von den andern Holzarten haben wir nur je einen Baum geprüft und wir konnten uns überdies in manchen Fällen nur solche von geringem Alter und Durchmesser verschaffen. So haben wir uns darauf beschränkt, blos die Ziffern, zu denen wir gelangten, anzugeben, ohne uns derselben zur Begründung praktischer Regeln zu bedienen, um somehr, als die Ziffern mancher Resultate z. B. für die Festigkeit der Zitteipappel, nicht mit den Ansichten über den bezüglichen Werth der betreffenden Holzarten übereinstimmen. Abgesehen von dieser Ziffer für die Zitterpappel kann man diese Holzarten nach ihren mechanischen Eigenschaften in folgender Reihe ordnen.

Ulme,

Esche,

Platane,

Erle,
Zitterpappel,

Ahorn,

Pappel. 


\section{FÜNFTER THEIL.}

\section{Schlussfolgerungen.}

A us allem was vorangeht, glauben wir folgende Schlüsse ziehen zu können.

1. Die Werthe der Elasticitätscoëfficienten und der Fortpflanzungsgeschwindigkeiten des Schalles, welche aus Schwingungen abgeleitet wurden, sind grösser als die durch Ausdehnung gefundenen Ziffern. Die Verhältnisse, zwischen den durch diese zwei Methoden gewonnenen Zahlen sind für die Bäume einer Holzart für was immer für einen Feuchtigkeitsgrad merklich dieselben; sie dienen dazı um den wirklichen Elasticitätscoëffi(ienten mittelst der wirklichen Schallgeschwindigkeit zu rechnen und umgekehrt.

2. Die durch Zug im Sinne der Faser erzeugte Ausdehnung besteht aus einem elastischen, den angewendeten Belastungen proportionalen und einem permanenten Theile, letzterer ist selbst bei verhältnissmässig kleinen Belastungen messbar und variirt nicht blos mit der Grösse dieser, sondern auch mit der Dauer der Wirksamkeit derselben.

3. Dieses Gesetz gilt gleichfalls für die Ausbiegungen, welche selbst sehr grosse Stiicke erleiden, wenn man sie an ihren Enden unterstuitzt und in der Mitte durch successive wachsende Gewichte belastet.

4. Die Elasticitätscoëfficienten, welche durch Ausbiegung von zwei Meter langen Walzen gefunden wurden, stimmen in Allgemeinen mit jenen mittleren Coëfficienten überein, welche durch Ausdehnungsversuche mit einer grossen Zahl von Stäben die dieser Walze angehörten unternommen wurden. Dennoch. 
findet diese Uebereinstimmung nicht bei den harzreichen Bäumen statt. Die Pfeilhöhen sind bei diesen viel grösser, als sie der Verlängerung der Stäbe nach sein sollten.

Wenn die Länge der Stäbe im Vergleiche zu ihrem Querschnitte eine sehr beträchtliche ist, so nähern sich die durch die Biegung erhaltenen Ziffern für was immer für Bäume sehr denjenigen, welche durch Ausdehnung ermittelt wurden.

5. Stäbe, welche in denselben Jahrringen in gleicher Höhe aber an verschiedenen Punkten entnommen wurden, zeigen in ihren mechanischen Eigenschaften wohl manche Abweichungen, aber zwischen den beobachteten Abweichungen und der ursprünglichen Position der Stäbe im Baume zu den Weltgegenden gibt es keine gesetzmässige Relation.

6. Die Dichte nimmt im Allgemeinen mit der Trockenheit und proportionirt zu dieser ab. Wenn man mit $d$ und $d^{\prime}$ die Dichten, bei den Feuchtigkeiten $h$ und $h^{\prime}$ bezeichnet, wobei $h>h^{\prime}$ ist, ferner mit c den Variationscoëfficienten für $1 \%$ Feuchtigkeit, und wenn man $h-h^{\prime}=H$ setzt, so hat man:

$$
d^{\prime}=d^{\prime}(1-c H) \text {. }
$$

Die Geschwindigkeit des Schalles nimmt mit der Trockenheit zu, u. z. proportional zu dieser.

Es seien $v$ und $v^{\prime}$ die Schallgeschwindigkeiten bei den Feuchtigkeiten $h$ und $h^{\prime}$ und $c^{\prime}$ der Variationscoëfficient der Schallgeschwindigkeit für $1 \%$ Wasserverlust, so wird man haben:

$$
\mathrm{v}^{\prime}=\mathrm{v}\left(1+\mathrm{c}^{\prime} \mathrm{H}\right) \text {. }
$$

Der Elasticitätscoëfficient nimmt mit der Trockenheit zu nach der Formel:

$$
\mathrm{E}^{\prime}=\mathrm{E}(1-\mathrm{cH})\left(\mathrm{I}+\mathrm{c}^{\prime} \mathrm{H}\right)^{2}
$$

Die Werthe von $\mathrm{c}$ und $\mathrm{c}^{\prime}$ sind für alle Holzarten, welche wir untersucht haben, derartige, das $\mathrm{E}^{\prime}$ gleichzeitig mit $\mathrm{H}$ wächst.

Die Elasticitätsgränze nimmt mit der Trockenheit zu und die Niaximalausdehnung nimmt ab. (Diese beiden Eigenschaften in ihrer gewöhnlichen Annahme vorausgesetzt.)

Die Festigkeit nimmt fast in allen Fällen mit den successiven Verlusten an Wasser in ziemlich starkem Verhältnisse zu.

Diese Thatsache ist indessen zu schwankender Natur, der Unmöglichkeit einer präcisen Bestimmung der Eigenschaft wegen, als dass man dieses Ergebniss dem Calcul unterwerfen könnte. Wenn die Trocknung bis auf $10 \%$ Wasser kiunstlich getrieben wurde, so wird das Holz derart gebrechlich, dass es nicht mehr möglich ist auch nur halbwegs präcise Versuche über den Bruch anzustellen. 
7. Die mechanischen Eigenschaften nehmen in constanter Weise, und manchmal in sehr starker Proportion vom Mittelpunkte gegen den Umfang hin zu, u. z. bei der Tanne in was immer für einem Alter, bei der Fichte, Weissbuche, Esche, Ulme, dem Ahorn, der Platane, Zitterpappel, Erle und zum Theil auch bei der Akazie.

Diese Zunahme scheint unabhängig vom Alter bei den harzreichen Bäumen zu sein, im Allgemeinen aber bei jenen Arten, deren Jahrringe immer für Flüssigkeiten durchdringlich bleiben. Bei der alten Eiche und Birke folgen die Eigenschaften einem umgekehrten Gang; d. h. nachdem sie bis zu einem Drittel des Radius zugenommen haben, nehmen sie bis zum Umfange hin wieder ab. Endlich findet man bei der Buche, bei jungen Bäumen cine Stcigerung der Eigenschaften, bei älteren Bäumen aber eine Abnahme, was darauf hindeuten würde, dass in den Bäumen, bei denen die älteren Jahrringe absterben, um den Kern zu bilden, diese Umbildung die Bewegung der Eigenschaften abändert.

8. Für jeden Jahrring an und für sich nehmen die mechanischen Eigenschaften mit der Höhe im Baume ab; dasselbe findet in den, auf die Axe senkrechten Richtungen statt. Fïr die Gesammtheit des Schaftes kann nur eine Abnahme mit der Höhe bei jenen Gattungen stattfinden, bei denen die schwächsten Jahrringe am Umfang liegen, und dies ist bei der Eiche der Fall. Aber bei den andern Gattungen, kann Verminderung, Gleichförmigkeit oder Vermehrung eintreten, je nachdem Verhältniss des Gesetzes der Zunahme vom Mittelpunkt gegen den Umfang, zu dem Gesetze der Abnahme in den Jahrringen von der Basis gegen den Gipfel.

Nichtsdestoweniger ist der Fall der Abnahme mit der Höhe im Allgemeinen der häufigste.

9. Die Verhältnisse zwischen Elasticität und Festigkeit im Sinne der Fasern und derselben Eigenschaften im Sinne des Radius und der Tangente variiren nicht merklich mit der Höhe in ein und demselben Baune oder in Bäumen derselben Art, aber wohl, wenn man die verschiedenen Arten untereinander in Vergleich zieht.

Wenn man die mittleren Elasticitäscoëfficienten und Festigkeiten im Sinne der Fasern als Einheiten annimmt, so finclet man die Elasticitätscoëfficienten im Sinne des Radius und der Tangente der Bäume durchschnittlich gleich $0 \cdot 165$ und 0.091 und für die Festigkeiten in diesen beiden Richtungen die Mittelzahlen $0 \cdot 163$ und $0 \cdot 159$. 
10. Man nimmt keinerlei regelmässige Beziehung zwischen der Dichte der Bäume, der Breite ihrer Jahrringe, der Exposition und der Bodenbeschaffenheit wahr.

11. Die Fällungszeit der Bäume scheint auf ihre mechanische Beschaffenheit zu influenciren.

12. Der Elasticitätscoëfficient und die Festigkeit nehmen in dem Masse ab, als das Alter der Bäume zunimmt.

13. Die Jahrringbreite kann als die erste Ursache weder der Differenzen die in einem und demselben Baume, noch jener die zwischen mehreren Individuen vorkommen, angesehen werden.

Es ist allerdings wahr, dass die graduelle Abnahme der Jahrringe bei der Tanne sich gleichmässig zur Vermehrung des Werthes der Eigenschaften, vom Mittelpunkt gegen den Umfang hin verhält, aber in jenen Fällen, wo das Gegentheil stattfindet, bleibt diese Vermehrung nicht minder merklich.

14. Die Hölzer, welche auf nördlichen, nordöstlichen und nordwestlichen Lagen und trockenem Boden erwachsen sind, haben immer einen grossen Elasticitätscoëfficienten und derselbe ist um so grösser, je mehr sich diese Umstände vereinigt finden; während die, auf nassem Boden erwachsenen Bäume geringere Coëfficienten darbieten.

Das gilt namentlich für die Rothbuche, bei welcher eben die Wirkung dieses Einflusses sehr auffallend ist.

15. Die Rothbuchen, welche auf Vogesenstandstein erwachsen sind, zeigen eine höhere Elasticität als jene vom bunten Sandstein und Muschelkalk.

16. In ein und demselben Baume gehen die verschiedenen mechanischen Eigenschaften fast immer parallel. So ist der dichteste Jahrring gewöhnlich derjenige, welcher auch die höchste Fortpflanzungsgeschwindigkeit des Schalles, den bedeutendsten Elasticitätscoëfficienten und die höchste Festigkeit besitzt; aber dieses schon in ein und demselben Baume zu wenig constante Verhältniss, auf dass es durch eine Formel ausgedrückt werden könnte, findet sich nur selten vor, wenn man untereinander verschiedene Bäume derselben Gattung vergleicht und es verschwindet gänzlich bei Bäumen verschiedener Art. 
Tabelle Nr. I.

Jebersicht der Resultate der Versuche, von verschiedenen Forschern, über die Dichtigkeit, Elasticität und Festigkeit der Hölzer.

\begin{tabular}{|c|c|c|c|c|c|c|}
\hline $\begin{array}{l}\text { Molz- } \\
\text { Gattung }\end{array}$ & $\begin{array}{l}\text { Qualitit und Ursprung } \\
\text { des Hol\%es }\end{array}$ & $\begin{array}{l}\text { Angewandte } \\
\text { Methode }\end{array}$ & 竎 & 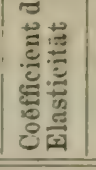 & 离 & Forscher \\
\hline Eiche & - & $\begin{array}{c}\text { Ausdehnung in } \\
\text { der Richtung der } \\
\text { Fasern }\end{array}$ & - & - & 9.810 & Rondelet \\
\hline$n$ & - & $n$ & 0.845 & - & 6.466 & Barlow \\
\hline$n$ & - & $"$ & 一 & - & 8.141 & Barlow \\
\hline$n$ & - & Biegung & - & 1012 & - & Duhamel \\
\hline- & - & $"$ & - & 1040 & - & Aubry \\
\hline$"$ & - $\quad-$ & $n$ & - & 969 & 一 & Aubry \\
\hline " & $\begin{array}{l}\text { Von einer Demolirung, } 25 \\
\text { Jahre seit dem Fällen . }\end{array}$ & $\eta$ & 0.732 & 1688 & - & Dupin \\
\hline$"$ & - $\quad-$ & $n$ & - & 1291 & 一 & Rondelet \\
\hline$n$ & Aus England $\cdot \cdots \cdot \cdot$ & $"$ & 0.969 & 614 & 4.982 & Barlow \\
\hline$"$ & $n \quad n \quad \cdots \cdot \cdots$ & $n$ & 0.934 & 1018 & 7.053 & n \\
\hline$n$ & n Danzig . . . . . . & $n$ & $|0.756|$ & 835 & 6.296 & $n$ \\
\hline$"$ & Von der adriatischen Küste & $"$ & 0.993 & 681 & 5.824 & $"$ \\
\hline$n$ & Aus Canada. . . . . & $"$ & 0.872 & 1507 & 7.452 & $"$ \\
\hline " & Altes Schiffsholz . • • & $\pi$ & 0.872 & 1205 & 8.350 & Ebbels und Tredgold \\
\hline$"$ & Junge, Kings Langley, Herts & $"$ & 0.863 & 1151 & $\mid 12,196$ & $"$ \\
\hline$n$ & Aus Beaulieu, Hants... & " & 0.616 & 740 & - & $n$ \\
\hline$"$ & $" n \quad$ ein and. Stïck & $"$ & 0.736 & 617 & - & $r$ \\
\hline r & Von einem alten Baume & $"$ & 0.625 & 500 & 5.516 & r \\
\hline " & Aus Riga. . • . . . & $"$ & 0.688 & 1131 & 9.033 & $"$ \\
\hline$n$ & n England $\ldots \ldots$ & $"$ & 0.748 & 1300 & 8.982 & $n$ \\
\hline$n$ & Grine englische . . . . & $n$ & 0.763 & 911 & 6.920 & $n$ \\
\hline$\Rightarrow$ & Aus Danzig, ausgetrocknet & $"$ & 0.755 & 1405 & 一 & $n$ \\
\hline$"$ & Quercus sossiliflora ... & $"$ & - & 1033 & - & $n$ \\
\hline$"$ & Quercus robur Frisch behauen, 4 Zoll im Ge- & $"$ & - & 1158 & - & $n$ \\
\hline$"$ & $\begin{array}{l}\text { vierte, } 20 \text { Fuss lang . . } \\
\text { Frisch bebauen, } 5 \text { Zoll im Ge. }\end{array}$ & $"$ & - & - & 6.944 & Buffon \\
\hline$n$ & $\begin{array}{l}\text { vierte, } 28 \text { Fuss lang . } \\
\text { Frisch behauen, } 6 \text { Zollim Ge. }\end{array}$ & 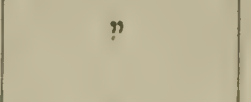 & - & - & 5.391 & " \\
\hline$"$ & $\begin{array}{l}\text { vierte, } 20 \text { Fuss lang . } \\
\text { Frisch behauen, } 7 \text { Zoll im Ge }\end{array}$ & $"$ & - & - & 5.772 & $"$ \\
\hline$"$ & $\begin{array}{l}\text { vierte, } 20 \text { Fuss lang } . \\
\text { Frisch behauen, } 8 \text { Zollim Ge- } \\
\text { vierte, } 20 \text { Fuss lang . . . }\end{array}$ & $"$ & 一 & - & $\begin{array}{l}6.091 \\
5.574\end{array}$ & $"$ \\
\hline$"$ & - $\quad-$ & $"$ & - & - & 7.322 & Belidor \\
\hline$"$ & - & $"$ & 一 & $\div$ & 7.259 & $n$ \\
\hline$"$ & - & $n$ & - & - & 7.124 & $"$ \\
\hline$n$ & - & Ausdehnung & - & 1340 & - & Minard u. Desormes \\
\hline
\end{tabular}




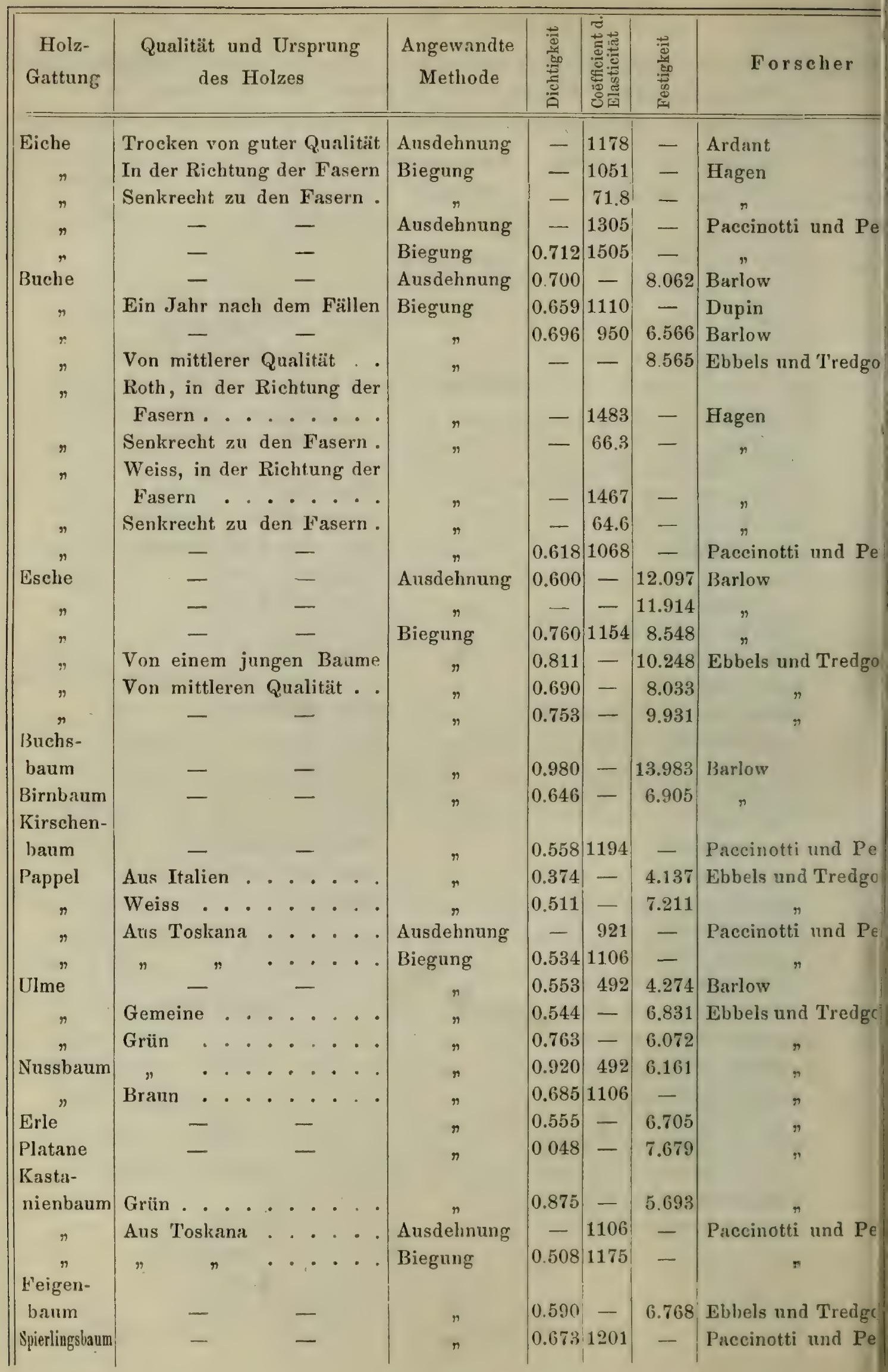




\begin{tabular}{|c|c|c|c|c|c|c|}
\hline $\begin{array}{l}\text { Holz- } \\
\text { Gattung }\end{array}$ & $\begin{array}{l}\text { Qualitait und Ursprung } \\
\text { des Holzes }\end{array}$ & $\begin{array}{c}\text { Angewandte } \\
\text { Methode }\end{array}$ & 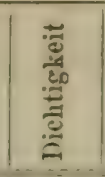 & 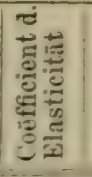 & 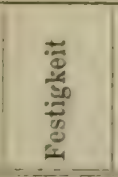 & Forscher \\
\hline $\begin{array}{l}\text { Olirenbanm } \\
\text { Akazien- }\end{array}$ & Aus Toscana . . . . & Biegung & 0.829 & 836 & - & Paccinotti und Peri \\
\hline bлиแ & Grün....... & $n$ & $0.820 \mid$ & - & 7.869 & Ebbels und Tredgold \\
\hline Weide & - & n & 0.405 & - & 4.618 & $r$ \\
\hline Birke & - & $"$ & 0.720 & - & 6.540 & $"$ \\
\hline Cypresse & Ein Jahr nach dem Fallen & $r$ & 0.664 & 1372 & - & Dupin \\
\hline Ileerhirschen. & Aus ''oscana $\ldots \ldots$ & $n$ & 0.502 & 1021 & - & Paccinotti und Peri \\
\hline baam & - & 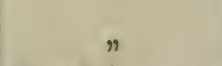 & 1.035 & 1313 & - & 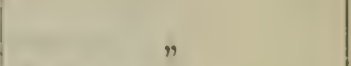 \\
\hline Acajou & - & Ausdehnung & 0.637 & - & 5.653 & Barlow \\
\hline$"$ & Von Spanien, trocken . & $n$ & 0.852 & - & 5.377 & Ebbels und Tredgold \\
\hline$"$ & Von Honduras, trocken. . & $n$ & 0.560 & - & 8.059 & $n$ \\
\hline$n$ & - & Biegung & 0.609 & 1424 & - & Paccinotti und Peri \\
\hline Ebenhol\% & - & $n$ & 1.125 & 2091 & - & $"$ \\
\hline Teak & - & Ausdehnung & 0.860 & - & 10.609 & Barlow \\
\hline$\eta$ & - & Biegung & 0.745 & 1693 & 10.386 & $"$ \\
\hline Poon & - & $n$ & 0.579 & - & 9.367 & $n$ \\
\hline Meleze & - & $"$ & 0.543 & 702 & - & $"$ \\
\hline $\begin{array}{l}\text { Calaba } \\
\text { (Schôtulatit) }\end{array}$ & - & $n$ & 0.579 & 1137 & - & $n$ \\
\hline Coder & Vom Libanon, trocken. & $"$ & 0.486 & - & 5.212 & Ebbels und Tredgold \\
\hline Tanne & $-\quad-$ & Ausdehnung & 0.600 & - & 9.039 & Barlow \\
\hline$n$ & - $\quad-$ & $"$ & - & 一 & 8.119 & r \\
\hline$"$ & $\begin{array}{l}\text { Von einer Demolirung, } 25 \\
\text { Jahre nach dem Fällen }\end{array}$ & Biegung & 0.443 & 1029 & - & Dupin \\
\hline$"$ & - & $"$ & - & 1350 & 一 & Rondelet \\
\hline " & Von Norwegen ..... & " & 0.660 & 859 & - & Barlow \\
\hline$"$ & Weisse von Christiana . & $"$ & 0.512 & 1268 & 8.678 & Ebbels und Tredgold \\
\hline$"$ & Weisse von Quebec ... & $n$ & $0.465 \mid$ & 875 & 7.211 & $r$ \\
\hline$"$ & Weisse aus Schottland.. & " & 0.529 & 845 & 7.363 & $\eta$ \\
\hline$"$ & Weisse aus England... & 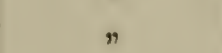 & 0.555 & 977 & 5.883 & r \\
\hline$"$ & Aus dem Walde von Mar. & r & 0.696 & 453 & 4.828 & Barlow \\
\hline$"$ & 一 & $n$ & 0.693 & 611 & 5.324 & $n$ \\
\hline$"$ & - & $n$ & 0.703 & 611 & 5.324 & $r$ \\
\hline$"$ & Aus den Vogesen... & Ausdehnung & - & 1615 & 一 & Ardant \\
\hline$n$ & $-\quad-$ & $r$ & 一 & 1188 & 一 & $"$ \\
\hline$"$ & Parallel zu den Fasern & Biegung & - & 1204 & - & Hagen \\
\hline$"$ & Seukrecht zu den Fasern. & $n$ & - & 25.3 & - & $"$ \\
\hline$n$ & - & Ausdehnung & - & 1155 & - & Pacciuotti und Peri \\
\hline$"$ & - $\quad-\quad-$ & Biegung & 0.484 & 940 & - & r \\
\hline$"$ & $\begin{array}{c}\text { Pinus abies in der Rich- } \\
\text { tung der Fasern .... }\end{array}$ & $"$ & 一 & 1330 & - & Hagen \\
\hline$"$ & Senkrecht zu den Fasern & $"$ & - & 15.7 & - & 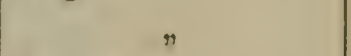 \\
\hline Fichte & Rothe oder schottische . & $n$ & 0.657 & 1290 & 5.658 & Barlow \\
\hline$n$ & Weisso oder von Neuengland & $"$ & 0.553 & 1046 & 4.650 & $"$ \\
\hline
\end{tabular}

Exner. Mechanischo Technologie des Molzes. 


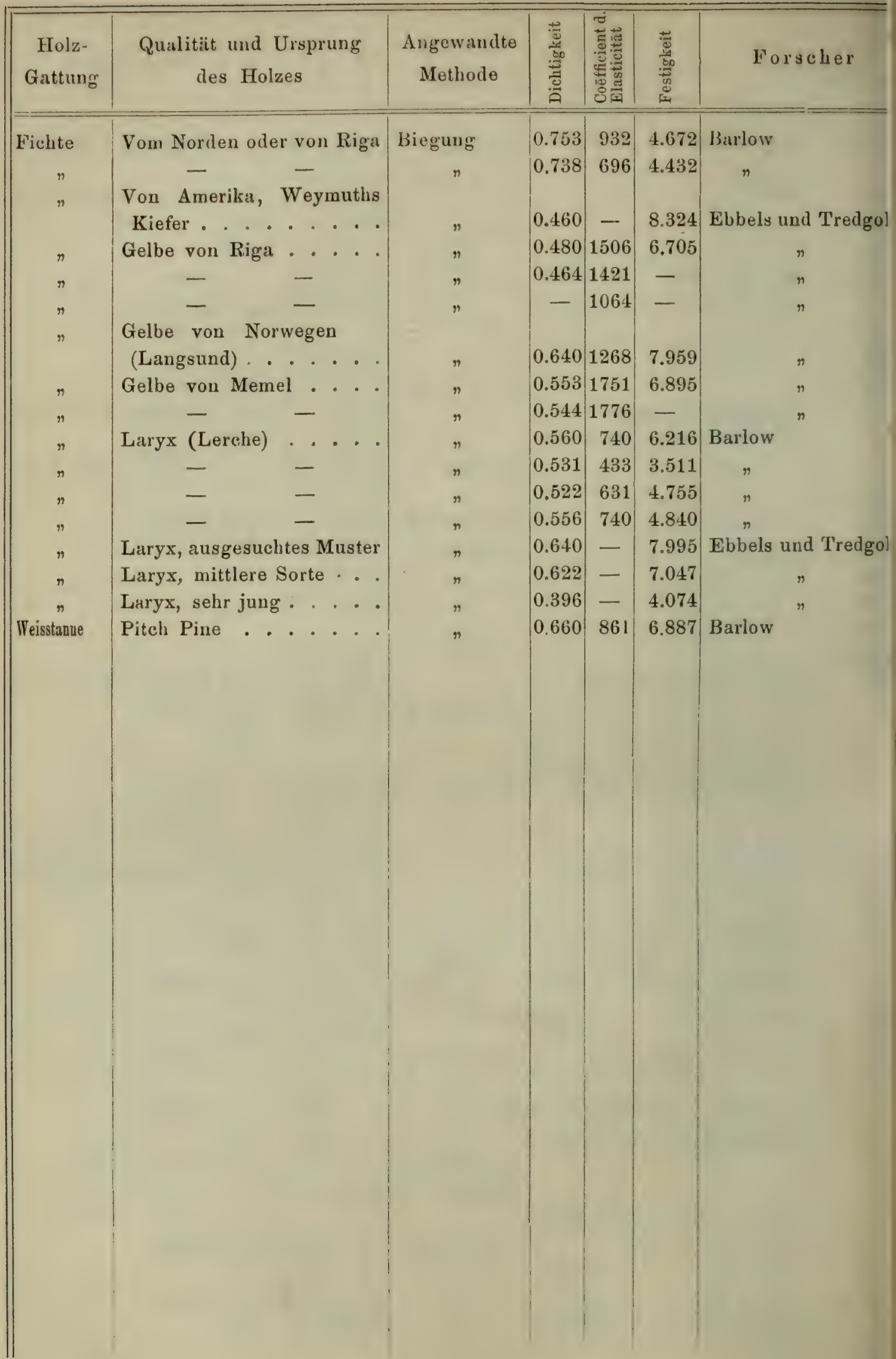




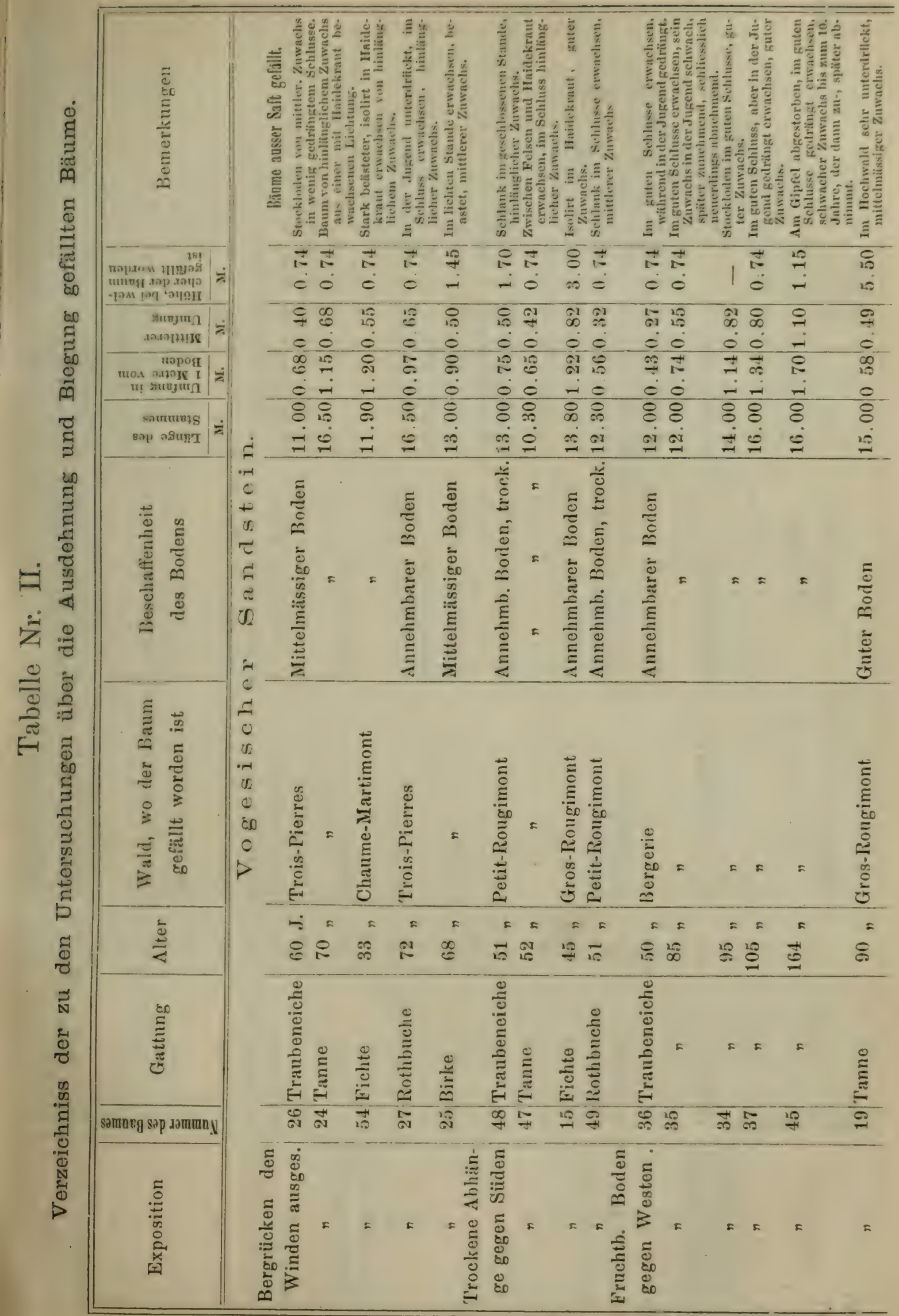




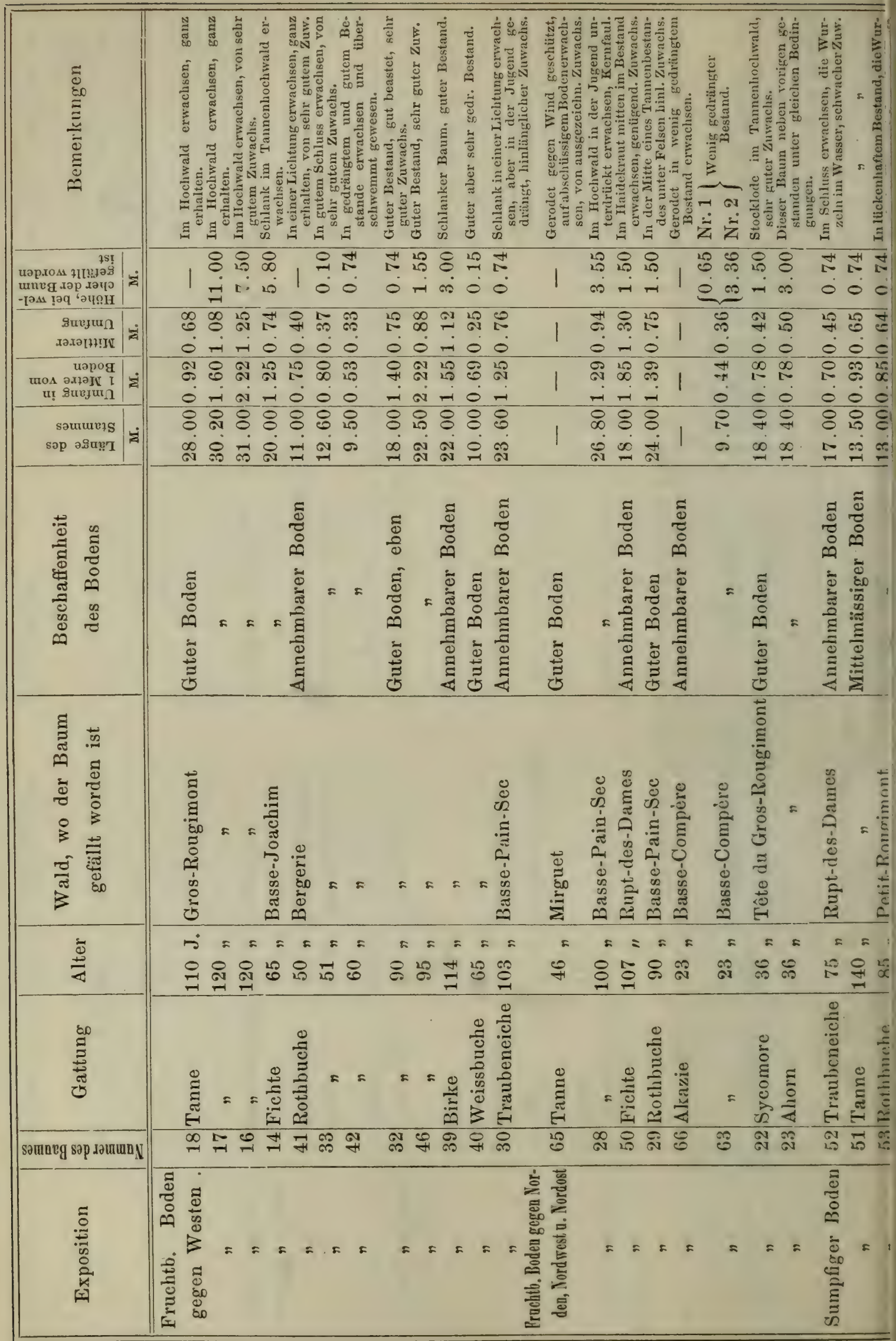




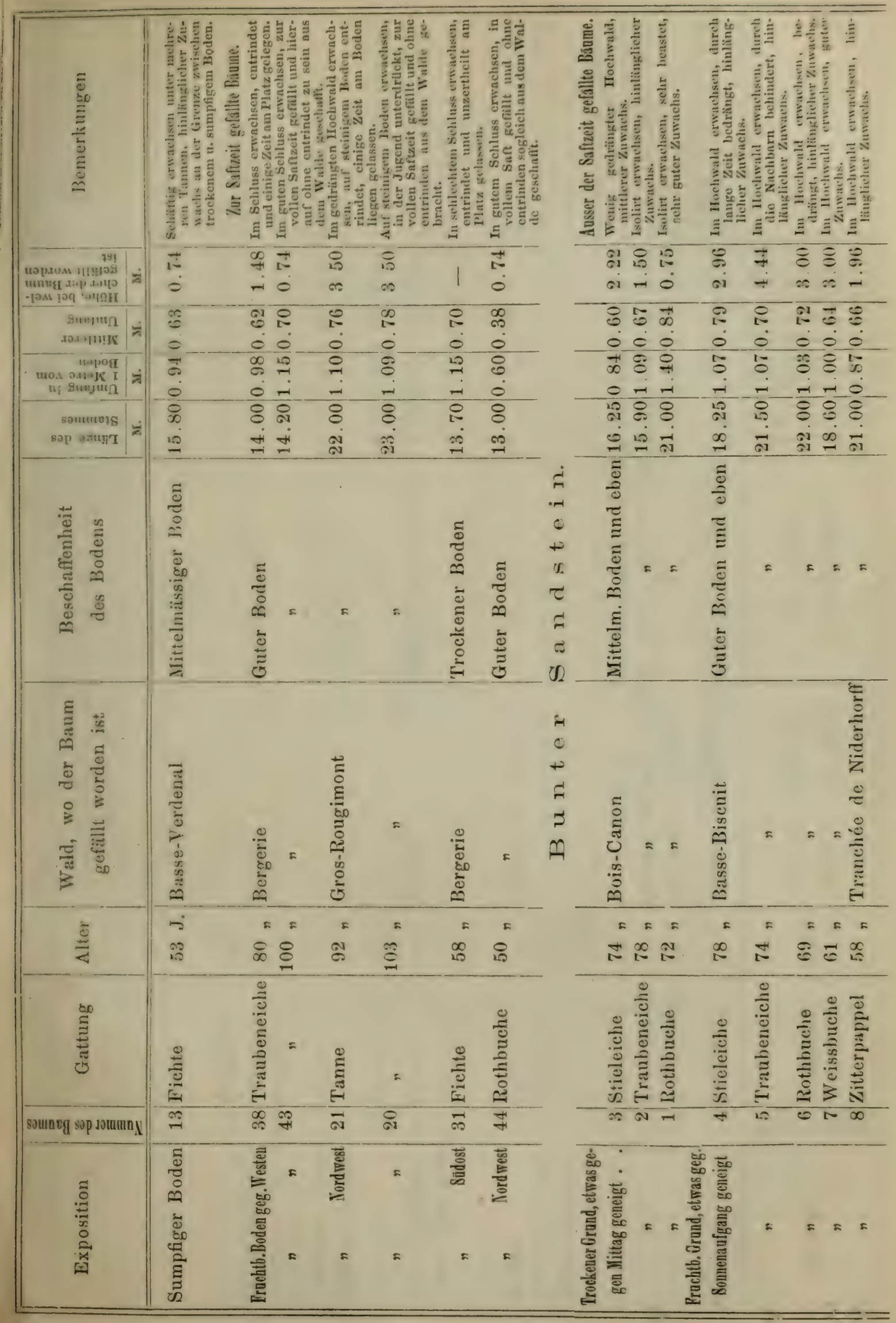




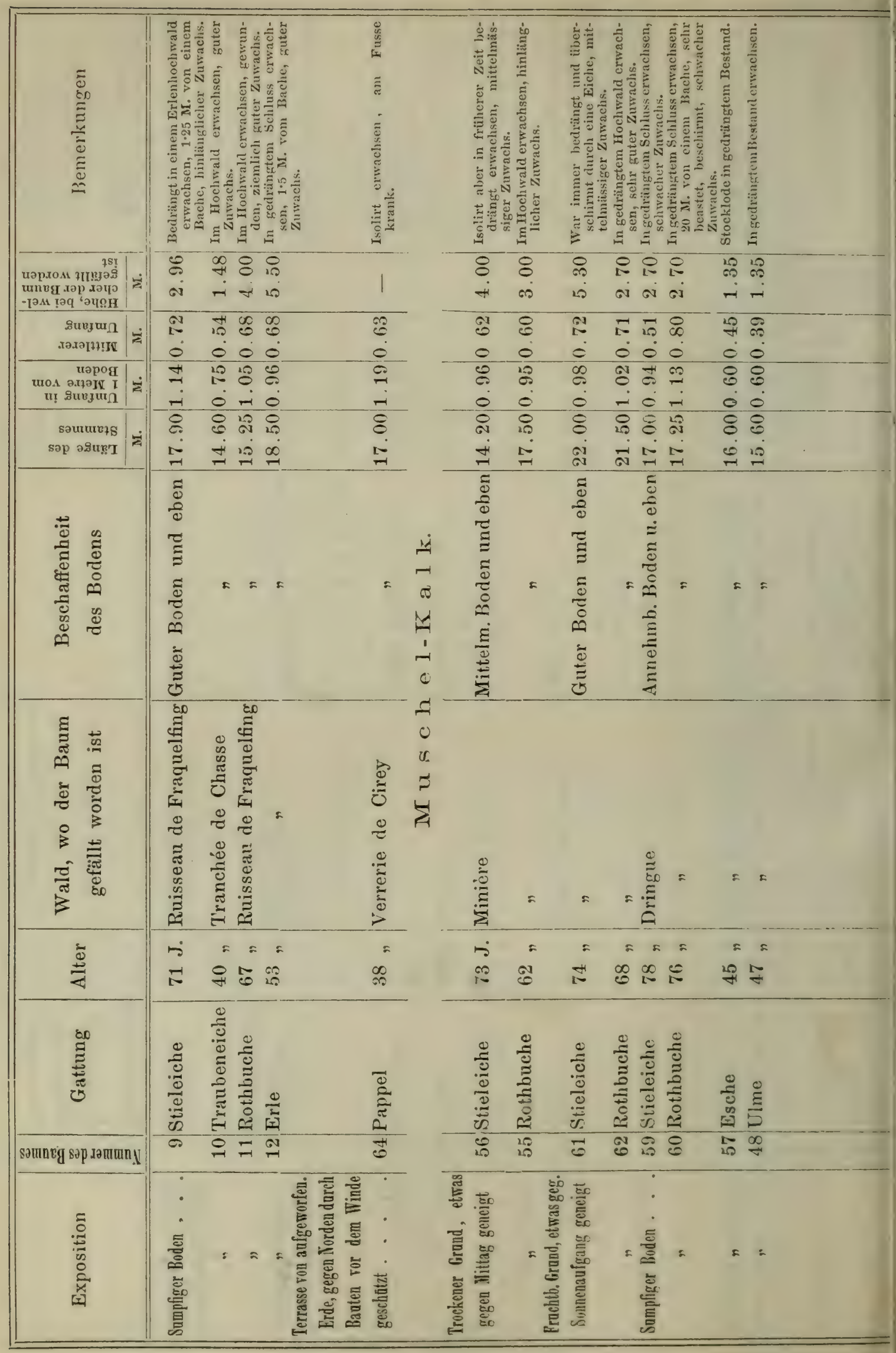




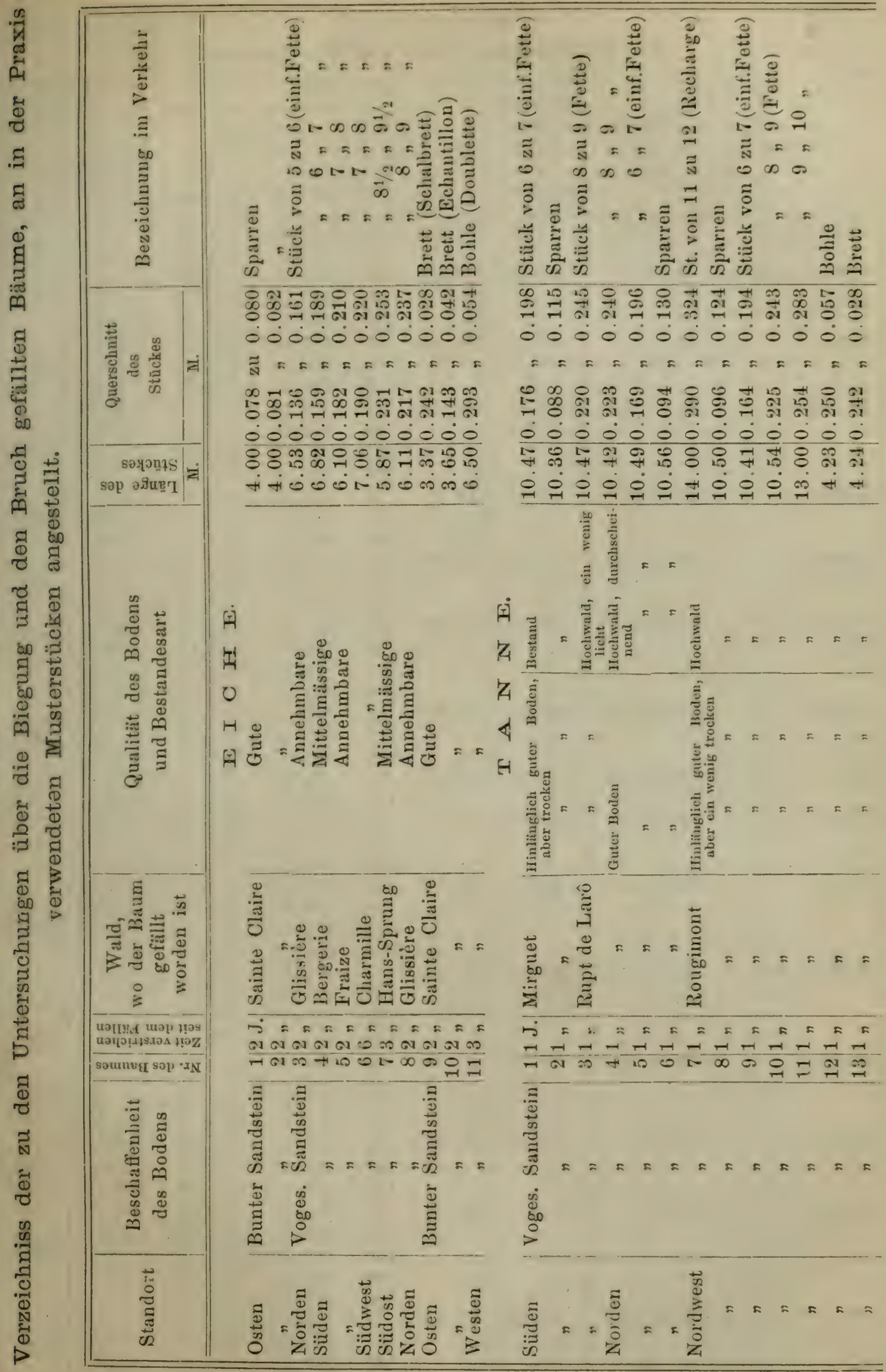




\section{- $72-$}

Untersuchungen über die Ausdehnung.

\begin{tabular}{|c|c|c|c|c|c|c|c|c|c|c|c|}
\hline \multirow{2}{*}{\multicolumn{4}{|c|}{ Gattung }} & \multirow{2}{*}{$\begin{array}{l}\text { Nummer } \\
\text { des } \\
\text { Stabes }\end{array}$} & \multirow{2}{*}{\begin{tabular}{|} 
Querschnitt \\
des \\
Stabes \\
in \\
$\square$ Millim.
\end{tabular}} & \multirow{2}{*}{ 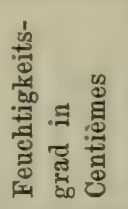 } & \multirow{2}{*}{ 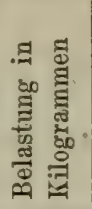 } & \multicolumn{2}{|c|}{$\begin{array}{l}\text { Entfernung der Marke } \\
\text { in Millimetern }\end{array}$} & \multirow{2}{*}{ 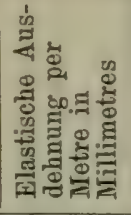 } & \multirow{2}{*}{$\begin{array}{l}\text { Coëfficient } \\
\text { der } \\
\text { Elasticitāt }\end{array}$} \\
\hline & & & & & & & & $\begin{array}{c}\text { mit } \\
\text { Belastung }\end{array}$ & $\begin{array}{c}\text { ohne } \\
\text { Belastung }\end{array}$ & & \\
\hline \multirow{2}{*}{\multicolumn{4}{|c|}{ Weissbuche }} & $7 \mathrm{~N}(2)$ & 48.88 & 37.22 & 40 & 814.87 & 814.16 & 0.872 & 9385 \\
\hline & & & & $8(1)$ & 5092 & 2189 & $\begin{array}{l}80 \\
40\end{array}$ & $\begin{array}{l}815.70 \\
805.84\end{array}$ & $\begin{array}{l}814,28 \\
805.09\end{array}$ & $\begin{array}{l}1.744 \\
0.932\end{array}$ & 9080 \\
\hline \multirow{2}{*}{\multicolumn{4}{|c|}{ Zitterpappel }} & (1) & 80.20 & 21.00 & 80 & 806.43 & 805.08 & 1.677 & 934.8 \\
\hline & & & & & & & 120 & 807.18 & 805.17 & 2.497 & \\
\hline \multirow[t]{3}{*}{ Erle . } & . . & . & - & $12(1)$ & 51.47 & 28.55 & 40 & 797.09 & 796.21 & 1.105 & \\
\hline & & & & & & & 80 & 798.06 & 796.40 & 2.084 & 718.3 \\
\hline \multirow{2}{*}{\multicolumn{2}{|c|}{ Sycomore }} & 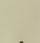 & & 00 & 0 & 31 & 120 & & 60 & 3.302 & \\
\hline & & • & & $22 \times(3)$ & 46.80 & 31.62 & $\begin{array}{l}40 \\
80\end{array}$ & $\begin{array}{l}778.50 \\
779.14\end{array}$ & $\begin{array}{r}777.89 \\
777.92\end{array}$ & $\begin{array}{l}0.784 \\
1.568\end{array}$ & \\
\hline & & & & & & & 120 & 77976 & 778.08 & 2.160 & 1136.6 \\
\hline & & & & & & & 160 & $780 \quad 49$ & 778.15 & 3.008 & \\
\hline \multirow[t]{3}{*}{ Ahorn } & . . & - & - & $23 \mathrm{~N}(3)$ & 47.02 & 13.79 & 40 & 814.55 & 813.75 & 0.983 & \\
\hline & & & & & & & 100 & 815.35 & 813.78 & 1.929 & 1026.1 \\
\hline & & & & & & & 160 & $816 \quad 50$ & 813.81 & 3.306 & \\
\hline \multirow[t]{2}{*}{ Eiche } & $=\cdot$ & - & - & $34(1)(1)$ & 46.37 & 10.96 & $\begin{array}{l}40 \\
80\end{array}$ & $\begin{array}{l}781.02 \\
781.58\end{array}$ & $\begin{array}{ll}780 & 49 \\
780 & 49\end{array}$ & $\begin{array}{l}0.679 \\
1.397\end{array}$ & 1205.6 \\
\hline & & & & & & & 140 & 782.55 & 780.54 & 2.575 & \\
\hline \multirow[t]{3}{*}{ Eiche } & - . & . & . & $34(1) \mathrm{E}(5)$ & 40.55 & 10.96 & 40 & 808.90 & 808.12 & 0.965 & \\
\hline & & & & & & & 80 & 809.51 & 808.18 & 1.646 & 1150.8 \\
\hline & & & & & & & 120 & 810.21 & 808.18 & 2.512 & \\
\hline \multirow[t]{3}{*}{ Eiche } & - . & . & . & $34(3) \mathrm{E}(2)$ & 46.55 & 16.63 & $\begin{array}{r}200 \\
40\end{array}$ & $\begin{array}{l}811.73 \\
811.32\end{array}$ & $\begin{array}{l}808.25 \\
810.72\end{array}$ & $\begin{array}{l}4306 \\
0.740\end{array}$ & \\
\hline & & & & & & & 100 & 812.10 & 810.71 & 1.715 & 1241.4 \\
\hline & & & & & & & 200 & 813.51 & 810.73 & 3.429 & \\
\hline Eiche & - & 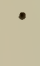 & - & $34(3) \mathrm{E}(4)$ & 45.88 & 16.63 & $\begin{array}{r}40 \\
100\end{array}$ & $\begin{array}{r}786.89 \\
787.98\end{array}$ & $\begin{array}{l}786.11 \\
786.21\end{array}$ & $\begin{array}{l}1.089 \\
2.486\end{array}$ & 853.6 \\
\hline \multirow[t]{2}{*}{ Birke } & . & - & . & $39(1)$ & 51.37 & 37.83 & 40 & 791.75 & 790.98 & 0.973 & \\
\hline & & & & & & & 80 & 792.38 & 791.06 & 1.669 & 888.4 \\
\hline \multirow{4}{*}{ Birke } & . & • & . & $39 \mathrm{~N}(3)$ & 4977 & & 120 & 793.14 & 791.07 & 2.617 & \\
\hline & & & & 39 in (0) & 42.78 & 10.67 & $\begin{array}{l}40 \\
80\end{array}$ & $\begin{array}{l}807.88 \\
808.30\end{array}$ & $\begin{array}{l}807.41 \\
807.45\end{array}$ & $\begin{array}{l}0.582 \\
1.053\end{array}$ & \\
\hline & & & & & & & 140 & 809.00 & 807.53 & 1.821 & 1709.7 \\
\hline & & & & & & & 200 & 809.85 & 807.56 & 2.836 & \\
\hline \multicolumn{2}{|c|}{ Rothbuche } & - & - & $41(1) \mathrm{E}(3)$ & 49.19 & 18.22 & 40 & 782.58 & 781.93 & 0.831 & \\
\hline & & & & & & & 80 & 783.22 & 781.94 & 1.637 & 1019.5 \\
\hline & & & & & & & $\begin{array}{l}120 \\
160\end{array}$ & $\begin{array}{r}783.85 \\
784.59\end{array}$ & $\begin{array}{l}781.93 \\
782.10\end{array}$ & $\begin{array}{l}2.324 \\
3.184\end{array}$ & \\
\hline Eiche & - . & . & . & $45 \mathrm{E}(3)$ & 98.68 & 19.53 & 80 & 814.26 & 813.68 & 0.713 & \\
\hline & & & & & & & 180 & 815.06 & 813.69 & 1.684 & 1095.0 \\
\hline & & & & & & & 300 & 815.93 & 813.72 & 2.716 & \\
\hline & & & & & & & 440 & 817.23 & 813.86 & 4.142 & \\
\hline Esche & $\cdot \cdot$ & . & . & $57(1)$ & 53.78 & 31.50 & 40 & 804.31 & 803.69 & 0.771 & \\
\hline & & & & & & & $\begin{array}{r}80 \\
120\end{array}$ & $\begin{array}{l}804.92 \\
805.71\end{array}$ & $\begin{array}{l}803.71 \\
803.80\end{array}$ & $\begin{array}{l}1.506 \\
2.376\end{array}$ & 959.1 \\
\hline Esche & . . & 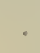 & . & 57 (1) & 52.77 & 10.54 & 40 & 815.35 & 814.96 & 0.479 & \\
\hline & & & & & & & 80 & 815.93 & 815.02 & 1.117 & \\
\hline & & & & & & & 140 & 816.72 & 815.00 & 2.110 & 1264.3 \\
\hline & & & & & & & 200 & 817.48 & 814.97 & 3.080 & \\
\hline Ulme & . . & 。 & - & $58 \mathrm{~N}(3)$ & 4726 & 33.26 & $\begin{array}{r}240 \\
40\end{array}$ & $\begin{array}{l}818.10 \\
797.16\end{array}$ & $\begin{array}{l}515.08 \\
796.40\end{array}$ & $\begin{array}{l}3.705 \\
0.954\end{array}$ & \\
\hline & & & & & & & 80 & 797.95 & 796.61 & 1682 & 1009.7 \\
\hline & & & & & & & $\begin{array}{l}140 \\
240\end{array}$ & $\begin{array}{l}799.20 \\
800.95\end{array}$ & $\begin{array}{r}796.80 \\
797.10\end{array}$ & $\begin{array}{l}3.012 \\
4830\end{array}$ & \\
\hline
\end{tabular}




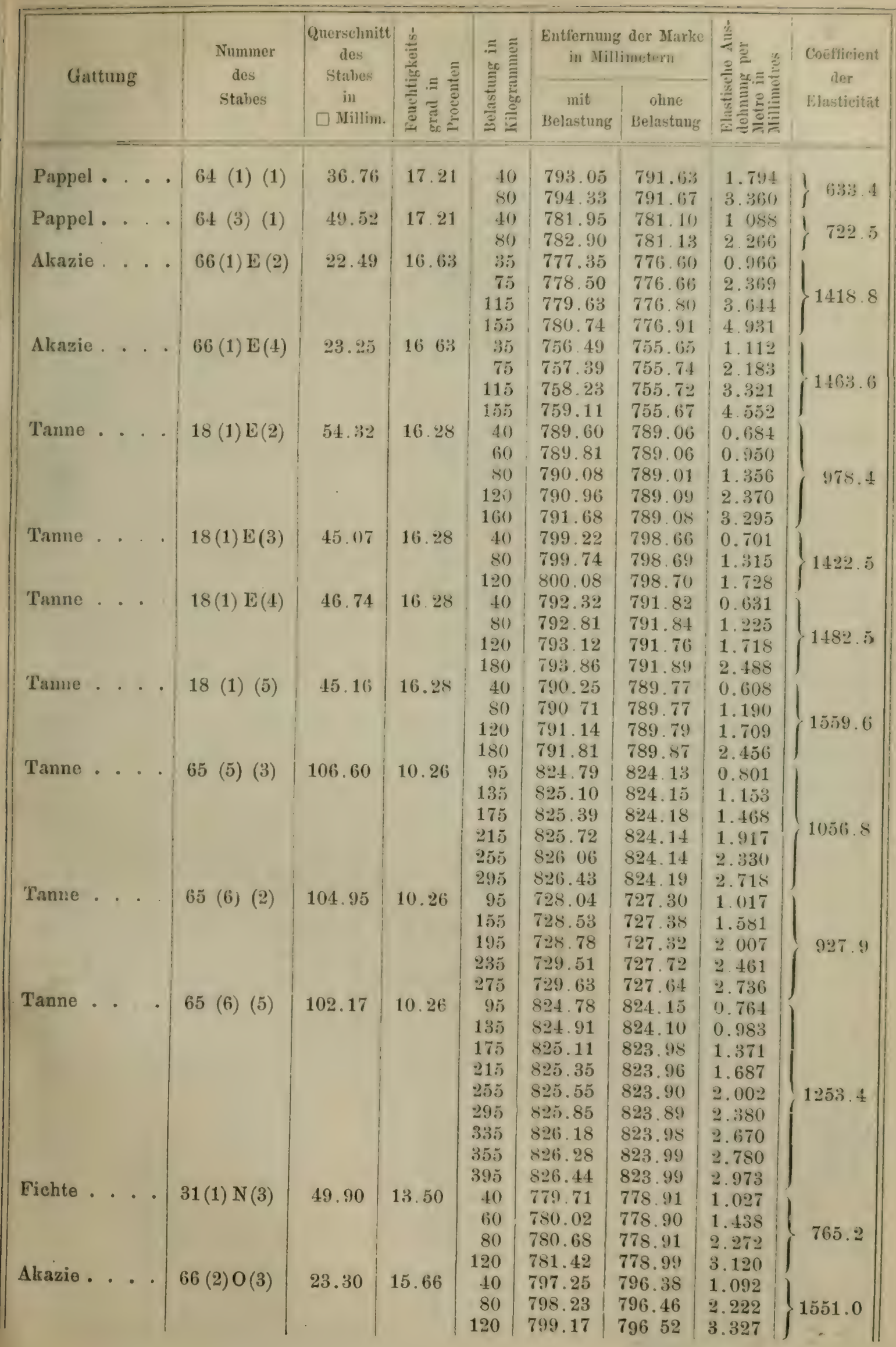




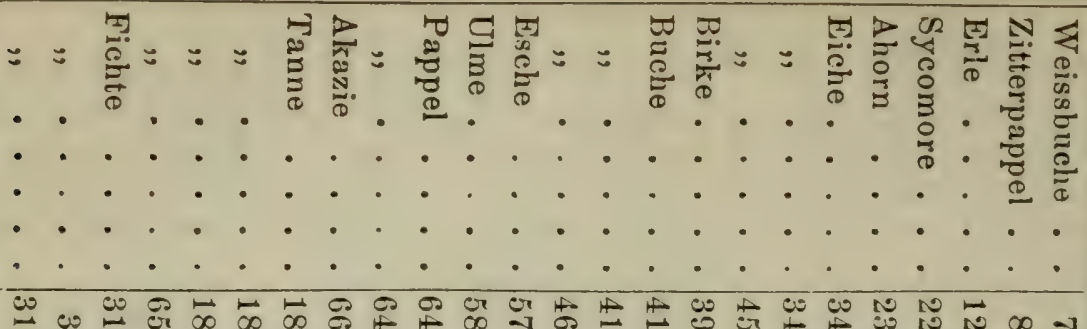

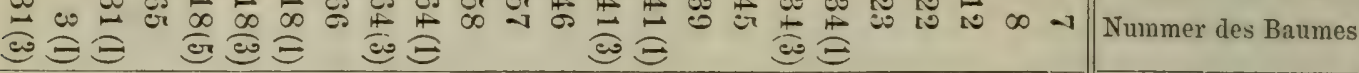

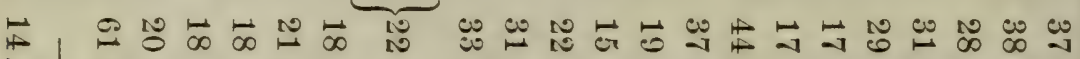

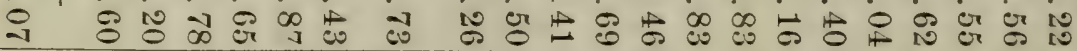

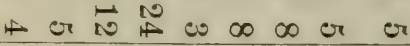

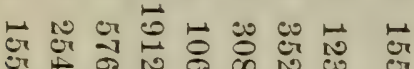
or

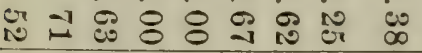

芹

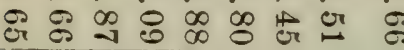

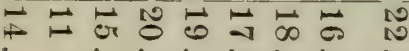

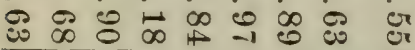
$\int_{\infty}^{\infty} \int_{\infty}^{\infty}$ is is 0 $\vec{\omega} \vec{\sigma} \overrightarrow{0} \overrightarrow{0} \vec{\sigma} \overrightarrow{0}$ is or is is co

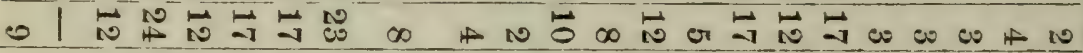

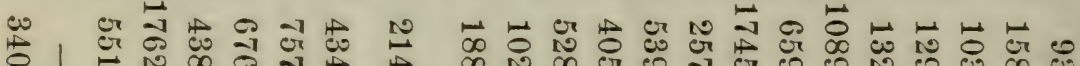
- |

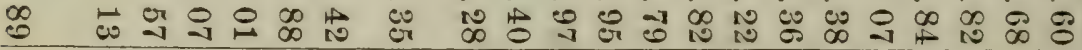
ש,

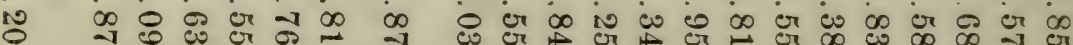

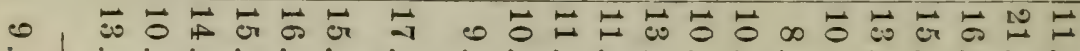

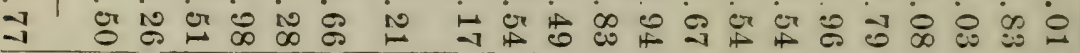

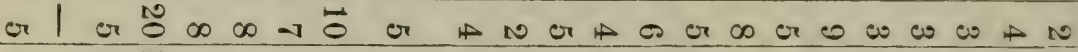

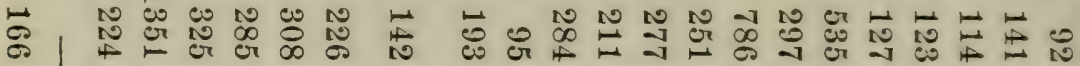

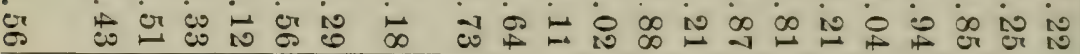

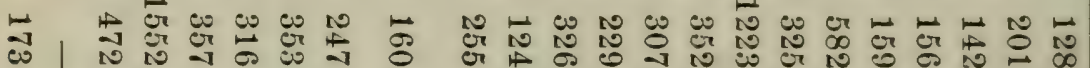

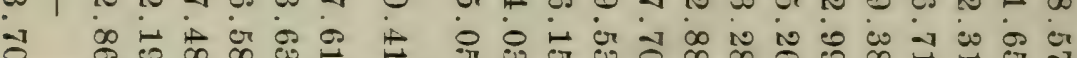

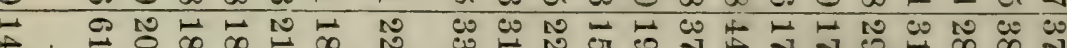
$-1,-\infty, \infty, \infty$ N

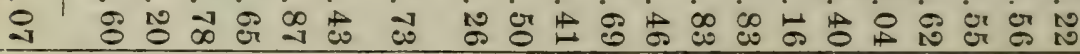

-

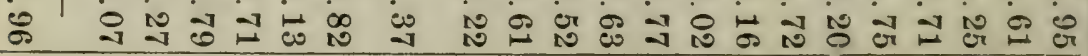
\begin{tabular}{l|l|}
$\begin{array}{l}\text { Quantität des } \\
\text { Wassers in } \\
\text { Procenten }\end{array}$ & 영 ․ㅕㄴ \\
\hline Zahl der Stäbe &
\end{tabular} Zahl der Stäbe Gewicht im
Momente der Untersuchung in Grammen Wassers in
Procenten

Zahl der Stiabe

Q

Quantitait des Wassers in
Procenten

Zahl der Stäbe

Gewicht im 


\section{Tabelle Nr. VI.}

Fouchtigkeitsgrad eines jedon Trummes zur Zeit der Vornahme der Biegungsversuche.

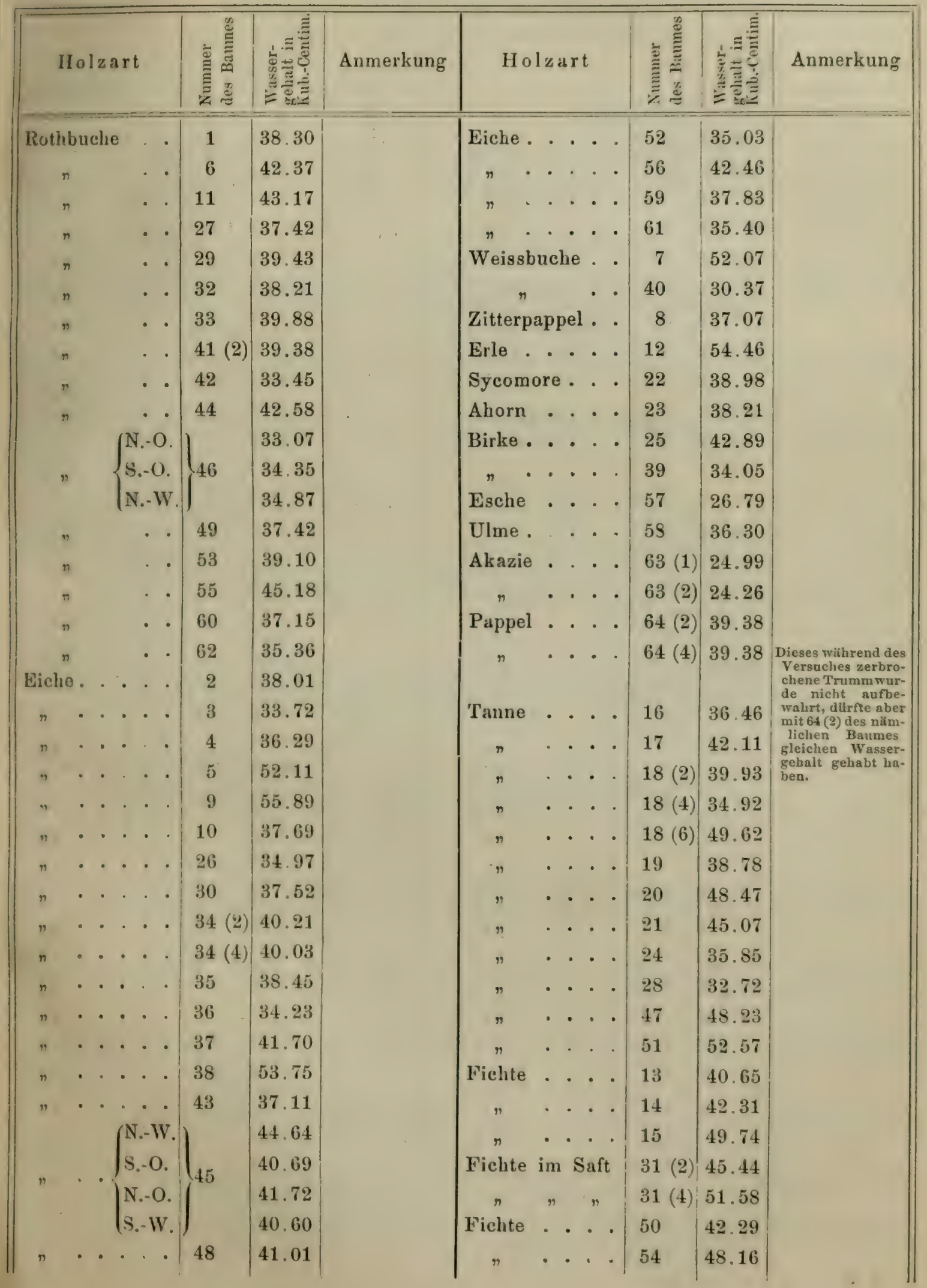


Tabelle

Beziehung zwischen der Fortpflanzungs-Geschwindigkeit des Schalles gungen und

\begin{tabular}{|c|c|c|c|c|c|c|c|c|c|c|c|c|c|}
\hline \multirow{2}{*}{ Art } & & \multirow{2}{*}{ 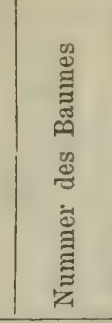 } & \multirow{2}{*}{ 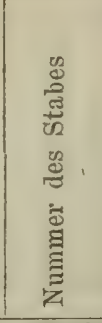 } & \multirow[b]{2}{*}{ 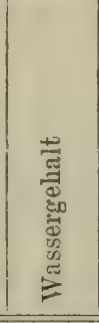 } & \multicolumn{2}{|c|}{$\begin{array}{l}\text { Elasticitäts- } \\
\text { coëfficienten } \\
\text { für die }\end{array}$} & \multirow[b]{2}{*}{ 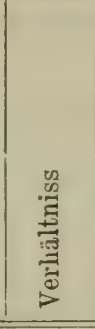 } & \multirow[b]{2}{*}{ 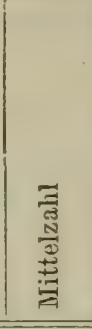 } & \multicolumn{2}{|c|}{$\begin{array}{c}\text { Schall- } \\
\text { geschwindig- } \\
\text { keiten } \\
\text { nach der }\end{array}$} & \multirow[b]{2}{*}{ 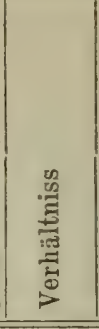 } & \multirow[b]{2}{*}{ 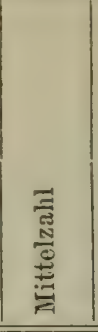 } & \multirow{2}{*}{ Anmerkung } \\
\hline & & & & & 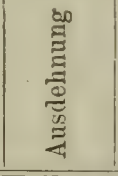 & 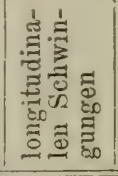 & & & 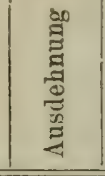 & 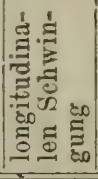 & & & \\
\hline Weissbuche & & 7 & N. [1] $\left[\begin{array}{l}1 \\
2]\end{array}\right.$ & 37.22 & $\begin{array}{l}636.8 \\
938.5\end{array}$ & $\begin{array}{l}795.6 \\
902.3\end{array}$ & $\begin{array}{l}1.249 \\
0.961\end{array}$ & 1.105 & $\begin{array}{r}8.86 \\
10.39\end{array}$ & $\begin{array}{r}9.90 \\
10.19\end{array}$ & $\begin{array}{l}\mathbf{1 . 1 1 7} \\
0.980\end{array}$ & 1.048 & \\
\hline Zitterpappe & & 8 & $\begin{array}{l} \\
\text { N. }[1] \\
\text { N. [2] }\end{array}$ & 38.56 & $\begin{array}{r}725.0 \\
1000.4 \\
1020.2\end{array}$ & $\begin{array}{r}671.2 \\
1009.8 \\
1220.0\end{array}$ & \begin{tabular}{|l|}
0.926 \\
1.009 \\
1.196
\end{tabular} & 1.044 & $\begin{array}{l}11.81 \\
13.80 \\
12.80\end{array}$ & $\begin{array}{l}11.36 \\
13.86 \\
14.00\end{array}$ & $\begin{array}{l}0.962 \\
1.004 \\
1.094\end{array}$ & 1.023 & \\
\hline Erle . & & 12 & N. [1] $\left[\begin{array}{l}{[1]} \\
{[3]}\end{array}\right.$ & 28.55 & $\begin{array}{l}718.3 \\
983.7\end{array}$ & $\begin{array}{r}720.2 \\
1118.8\end{array}$ & $\begin{array}{l}1.003 \\
1.137\end{array}$ & 1.070 & $\begin{array}{l}11.15 \\
12.94\end{array}$ & $\begin{array}{l}11.17 \\
13.80\end{array}$ & $\begin{array}{l}1.002 \\
1.066\end{array}$ & 1.034 & \\
\hline Sycomore & . & 22 & N. $\left[\begin{array}{l}1 \\
3\end{array}\right]$ & 31.62 & $\begin{array}{r}706.9 \\
1136.7\end{array}$ & $\begin{array}{r}761.2 \\
1400.4\end{array}$ & $\begin{array}{l}1.077 \\
1.232\end{array}$ & 1.154 & $\mid \begin{array}{l}10.78 \\
12.20\end{array}$ & $\begin{array}{l}11.18 \\
13.54\end{array} \mid$ & $\begin{array}{l}1.038 \\
1.110\end{array}$ & 1.074 & \\
\hline Ahorn. . & & 23 & N. $\left[\begin{array}{l}{[1]} \\
{[3]}\end{array}\right.$ & 13.79 & $\begin{array}{r}983.0 \\
1026.1\end{array}$ & $\begin{array}{l}1002.2 \\
1146.3\end{array}$ & $\begin{array}{l}1.019 \\
1.117\end{array}$ & 1.068 & $\begin{array}{l}12.61 \\
12.31\end{array}$ & $\begin{array}{l}12.74 \\
13.01\end{array}$ & $\begin{array}{l}1.010 \\
1.057\end{array}$ & 1.033 & \\
\hline Eiche . & & 34 (1) & O. $\begin{array}{l}2] \\
{[4]} \\
{[5]}\end{array}$ & 19.08 & $\begin{array}{r}1111.8 \\
1278.3 \\
883.8\end{array}$ & $\begin{array}{r}1236.8 \\
1570.8 \\
1086.9\end{array}$ & $\begin{array}{l}1.112 \\
1.229 \\
1.230\end{array}$ & 1.190 & $\begin{array}{l}11.74 \\
11.75 \\
11.74\end{array}$ & $\begin{array}{l}12.38 \\
13.02 \\
13.01\end{array}$ & $\begin{array}{l}1.054 \\
1.108 \\
1.108\end{array}$ & 1.090 & \\
\hline Eiche & & $34(3)$ & O. $\begin{array}{l}2] \\
{[3]} \\
{[4]}\end{array}$ & 16.63 & $\begin{array}{r}1241.4 \\
1056.7 \\
853.6\end{array}$ & $\begin{array}{l}1445.7 \\
1088.2 \\
1106.8\end{array}$ & $\begin{array}{l}1.165 \\
1.030 \\
1.297\end{array}$ & 1.164 & $\left|\begin{array}{l}11.88 \\
11.61 \\
10.67\end{array}\right|$ & $\begin{array}{l}12.82 \\
11.79 \\
12.15\end{array}$ & $\begin{array}{l}1.079 \\
1.015 \\
1.138\end{array}$ & 1.077 & \\
\hline Eiche. . & & 45 & O. $\begin{array}{l}{[3]} \\
{[4]} \\
{[5]}\end{array}$ & 19.53 & $\begin{array}{r}1095.1 \\
441.0 \\
505.1\end{array}$ & $\begin{array}{l}924.2 \\
478.4 \\
585.0\end{array}$ & $\begin{array}{l}0.844 \\
1.085 \\
1.158\end{array}$ & 1.029 & $\begin{array}{r}11.76 \\
8.66 \\
883\end{array}$ & $\begin{array}{r}10.81 \\
9.02 \\
9.51\end{array}$ & $\begin{array}{l}0.919 \\
1.041 \\
1.077\end{array}$ & 1.012 & \\
\hline Birke. . & & 39 & N. $\begin{array}{l}{[1]} \\
{[3]} \\
{[5]}\end{array}$ & 37.83 & $\begin{array}{r}888.4 \\
1178.4 \\
699.0\end{array}$ & $\begin{array}{r}1130.6 \\
1298.7 \\
976.7\end{array}$ & $\begin{array}{l}1.272 \\
1.102 \\
1.397\end{array}$ & 1.257 & $\begin{array}{r}10.04 \\
11.48 \\
8.83\end{array}$ & $\begin{array}{l}11.33 \\
12.05 \\
10.44\end{array}$ & $\begin{array}{l}1.128 \\
1.049 \\
1.182\end{array}$ & 1.120 & \\
\hline Rothbuche & & 41 (1) & O. $\begin{array}{l}{[3]} \\
{[4]}\end{array}$ & 18.22 & $\begin{array}{r}1018.9 \\
985.6\end{array}$ & $\begin{array}{r}950.6 \\
1083.7\end{array}$ & $\begin{array}{l}0.933 \\
1.099\end{array}$ & 1.016 & $\mid \begin{array}{l}11.15 \\
10.91\end{array}$ & $\begin{array}{l}10.77 \\
11.45\end{array}$ & $\left|\begin{array}{l}0.966 \\
1.0+9\end{array}\right|$ & 1.007 & \\
\hline Rothbuche & . & $41(3)$ & o. [3] & 16.50 & 806.1 & 799.0 & 0.991 & 0.991 & 9.76 & 9.72 & 0.996 & 0.996 & \\
\hline Rothbuche & & 46 & O. [2] & 13.92 & $\begin{array}{r}895.7 \\
1164.2 \\
899.5\end{array}$ & $\begin{array}{l}1301.7 \\
1260.4 \\
1214.1\end{array}$ & $\begin{array}{l}1.453 \\
1.083 \\
1.350\end{array}$ & 1.295 & $\begin{array}{l}10.47 \\
12.10 \\
10.89\end{array}$ & $\begin{array}{l}12.62 \\
12.60 \\
12.65\end{array}$ & $\begin{array}{l}1.205 \\
1.041 \\
1.162\end{array}$ & 1.136 & \\
\hline Esche . & & 57 & N. $\begin{array}{l}{[1]} \\
{[2]}\end{array}$ & 31.50 & $\begin{array}{r}959.0 \\
1171.3\end{array}$ & $\begin{array}{l}1216.7 \\
1476.4\end{array}$ & $\begin{array}{l}1.268 \\
1.260\end{array}$ & 1.264 & $\begin{array}{l}11.58 \\
11.84\end{array}$ & $\begin{array}{l}13.05 \\
13.30\end{array}$ & $\begin{array}{l}1.127 \\
1.123\end{array}$ & 1.125 & \\
\hline Ulme. & & 58 & N. $\left[\begin{array}{l}{[1]} \\
\text { N. }[3]\end{array}\right.$ & 33.26 & $\begin{array}{r}435.1 \\
1009.5\end{array}$ & $\begin{array}{r}558.2 \\
1248.7\end{array}$ & $\begin{array}{l}1.283 \\
1.237\end{array}$ & 1.260 & $\begin{array}{r}8.02 \\
11.25\end{array}$ & $\begin{array}{r}9.08 \\
12.51\end{array}$ & \begin{tabular}{|l|}
1.132 \\
1.112
\end{tabular} & 1.122 & \\
\hline
\end{tabular}


Nr. VII.

und dem Elasticitätscoëfficienten, ermittelt durch longitudinale SchwinAusdebnungen.

\begin{tabular}{|c|c|c|c|c|c|c|c|c|c|c|c|c|c|}
\hline \multirow{2}{*}{ Art } & \multirow{2}{*}{ 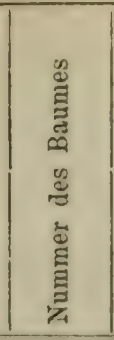 } & \multirow{2}{*}{ 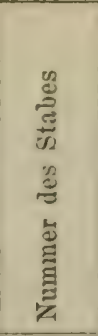 } & \multirow[b]{2}{*}{ 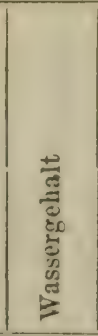 } & \multicolumn{2}{|c|}{$\begin{array}{l}\text { Elasticitāts- } \\
\text { coëfficienten } \\
\text { für dis }\end{array}$} & \multirow[b]{2}{*}{ 总 } & \multirow[b]{2}{*}{ 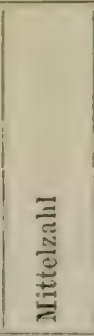 } & \multicolumn{2}{|c|}{\begin{tabular}{|c|} 
Schall- \\
geschwindig- \\
keiten \\
nach der
\end{tabular}} & \multirow[b]{2}{*}{ 王 } & \multirow[b]{2}{*}{ 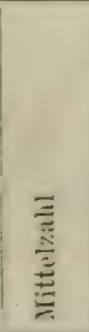 } & \multicolumn{2}{|c|}{ 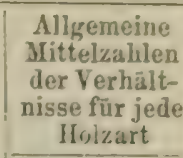 } \\
\hline & & & & 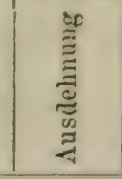 & 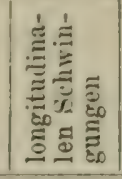 & & & 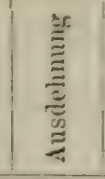 & 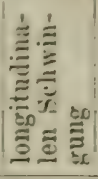 & & & 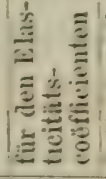 & 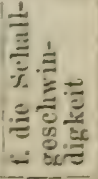 \\
\hline - & - & - & - & - & $\overline{-}$ & - & & I & - & I & & 1.105 & 1.048 \\
\hline Zitterpappel & 8 & $\begin{array}{l}{[1]} \\
\text { N. }[4]\end{array}$ & 21.83 & $\begin{array}{r}935.1 \\
1437.4\end{array}$ & $\begin{array}{r}871.7 \\
1615.0\end{array}$ & \begin{tabular}{|l|}
0.932 \\
1.123
\end{tabular} & 1.027 & $\begin{array}{l}13.80 \\
15.57\end{array}$ & $\begin{array}{l}13.33 \\
16.50\end{array}$ & $\begin{array}{l}0.966 \\
1.059\end{array}$ & 1.012 & 1.035 & 1.017 \\
\hline Erle . & 12 & N. [3] & 16.03 & 1086.2 & 1274.1 & 1.173 & 1.173 & 13.84 & 14.99 & 1.083 & 1.083 & 1.121 & 1.058 \\
\hline Sycomore & 22 & N. [3] & 15.08 & 1294.8 & 1457.1 & 1125 & 1.125 & 13.72 & 14.56 & 1.061 & 1.061 & 1.139 & 1.067 \\
\hline & - & - & 二 & $\overline{-}$ & - & - & & $\overline{-}$ & - & $=$ & & 1.068 & 1.033 \\
\hline Eiche & 34 (1) & O. $\begin{array}{l}{[1]} \\
{[3]} \\
{[3]} \\
{[4]} \\
{[5]}\end{array}$ & 10.96 & $\begin{array}{l}1205.6 \\
1375.2 \\
1241.3 \\
1517.0 \\
1151.0\end{array}$ & $\begin{array}{l}1121.5 \\
1425.8 \\
1321.5 \\
1520.0 \\
1193.9\end{array}$ & $\begin{array}{l}0.930 \\
1.037 \\
1.065 \\
1.002 \\
1.037\end{array}$ & 1.014 & \begin{tabular}{|l|}
11.96 \\
12.46 \\
12.02 \\
12.88 \\
12.26
\end{tabular} & \begin{tabular}{|l|}
11.55 \\
12.69 \\
12.40 \\
12.89 \\
12.48
\end{tabular} & $\begin{array}{l}0.965 \\
1.018 \\
1.032 \\
1.000 \\
1.018\end{array}$ & 1.007 & 1.102 & 1.048 \\
\hline Eiche . & $34(3)$ & O. $\left.\begin{array}{l}{[1]} \\
{[2]} \\
{[3]} \\
{[4]}\end{array}\right]$ & 8.54 & $\begin{array}{l}1488.1 \\
1390.2 \\
1246.2 \\
1060.2\end{array}$ & $\begin{array}{l}1488.6 \\
1478.7 \\
1341.3 \\
1255.5\end{array}$ & $\begin{array}{l}1.000 \\
1.064 \\
1.076 \\
1.184\end{array}$ & 1.081 & $\begin{array}{l}13.38 \\
12.65 \\
12.73 \\
12.15\end{array}$ & $\begin{array}{l}13.38 \\
13.05 \\
13.21 \\
13.22\end{array}$ & $\begin{array}{l}1.000 \\
1.032 \\
1.038 \\
1.088\end{array}$ & 1.039 & 1.122 & 1.058 \\
\hline Eiche & 45 & O. $\left.\begin{array}{l}{[1]} \\
{[2]} \\
{[3]} \\
{[4]} \\
{[5]}\end{array}\right]$ & 10.54 & $\begin{array}{r}640.2 \\
905.5 \\
1194.1 \\
9741 \\
556.6\end{array}$ & \begin{tabular}{|r|}
1071.0 \\
1239.0 \\
1292.0 \\
552.0 \\
801.0
\end{tabular} & $\begin{array}{l}1.673 \\
1.368 \\
1.082 \\
0.567 \\
1.439\end{array}$ & 1.226 & $\begin{array}{r}9.38 \\
10.39 \\
11.79 \\
12.79 \\
8.54\end{array}$ & \begin{tabular}{r|r|r}
12.14 & \\
12.15 \\
12.27 \\
9.63 \\
10.25
\end{tabular} & $\begin{array}{l}1.294 \\
1.169 \\
1.041 \\
0.753 \\
1.200\end{array}$ & 1.091 & 1.127 & 1.051 \\
\hline Birlke . & 39 & N. [1] $[3]$ & 10.67 & $\begin{array}{l}1288.9 \\
1709.7\end{array}$ & $\begin{array}{l}1558.1 \\
1926.9\end{array}$ & $\begin{array}{l}1.209 \\
1.127\end{array}$ & 1.168 & $\begin{array}{l}14.02 \\
14.52\end{array}$ & \begin{tabular}{|l|}
15.41 \\
15.41
\end{tabular} & $\begin{array}{l}1.099 \\
1.061\end{array}$ & 1.080 & 1.212 & 1.100 \\
\hline Rothbuche & $41(1)$ & O. [2] $\left.\begin{array}{r}{[3]} \\
{[4]}\end{array}\right]$ & 13.94 & $\begin{array}{r}982.1 \\
986.5 \\
1060.9\end{array}$ & $\begin{array}{l}1020.5 \\
1070.1 \\
1225.8\end{array}$ & $\begin{array}{l}1.039 \\
1.085 \\
1.155\end{array}$ & 1.093 & $\begin{array}{l}10.96 \\
11.07 \\
11.57\end{array}$ & $\begin{array}{l}11.17 \\
11.53 \\
12.44\end{array}$ & $\begin{array}{l}1.019 \\
1.041 \\
1.075\end{array}$ & 1.045 & 1.054 & 1.026 \\
\hline Rothbuche & $41(3)$ & N. [2] & 11.83 & $\begin{array}{l}1200.4 \\
1142.5\end{array}$ & $\begin{array}{l}1226.7 \\
1287.1\end{array}$ & $\begin{array}{l}1.022 \\
1.126\end{array}$ & 1.07 .4 & $\begin{array}{l}11.43 \\
11.97\end{array}$ & $\begin{array}{l}11.56 \\
12.70\end{array}$ & $\left|\begin{array}{l}1.011 \\
1.061\end{array}\right|$ & 1.036 & 1.032 & 1.016 \\
\hline Rothbuche & 46 & O. [2] $\left.\begin{array}{r}2 \\
{[3]} \\
{[4]}\end{array}\right]$ & 11.49 & $\begin{array}{l}1134.2 \\
1213.5 \\
1072.0\end{array}$ & $\begin{array}{l}1510.7 \\
1359.9 \\
1193.9\end{array}$ & $\begin{array}{l}1.332 \\
1.121 \\
0.114\end{array}$ & 1.189 & $\begin{array}{l}11.76 \\
12.38 \\
11.98\end{array}$ & \begin{tabular}{|l|}
13.57 \\
13.11 \\
12.64
\end{tabular} & $\begin{array}{l}1.154 \\
1.059 \\
1.055\end{array}$ & 1.089 & 1.242 & 1.112 \\
\hline Esche & 57 & N. [1] $\left[\begin{array}{l}{[3]} \\
{[}\end{array}\right]$ & 1054 & $\begin{array}{l}1264.3 \\
1280.4\end{array}$ & $\begin{array}{l}1409.2 \\
1723.0\end{array}$ & $\begin{array}{l}1.115 \\
1.345\end{array}$ & 1.229 & $\begin{array}{l}13.92 \\
12.80\end{array}$ & $\begin{array}{l}14.70 \\
14.85\end{array}$ & $\begin{array}{l}1.056 \\
1.160\end{array}$ & 1.1 & 1.246 & 1.116 \\
\hline Ulme . & 58 & N. [3] & \begin{tabular}{|l|}
9.17 \\
\end{tabular} & 1403.0 & 1530.2 & 1.090 & 1.090 & 13.85 & 14.46 & 1.044 & 1.044 & 1.175 & 1.083 \\
\hline
\end{tabular}




\begin{tabular}{|c|c|c|c|c|c|c|c|c|c|c|c|c|c|}
\hline \multirow[b]{2}{*}{$\mathrm{Ar}$} & & \multirow{2}{*}{ 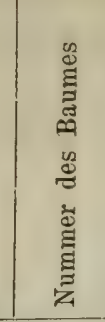 } & \multirow{2}{*}{ 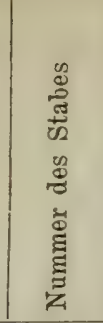 } & \multirow[b]{2}{*}{ 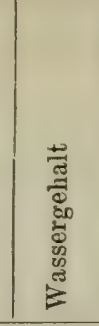 } & \multicolumn{2}{|c|}{$\begin{array}{l}\text { Elasticitäts- } \\
\text { coëfficienten } \\
\text { für die }\end{array}$} & \multirow[b]{2}{*}{ 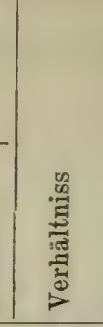 } & \multirow[b]{2}{*}{ 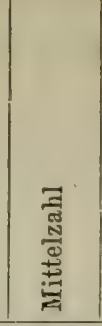 } & \multicolumn{2}{|c|}{$\begin{array}{l}\text { Schall- } \\
\text { geschwindig- } \\
\text { keiten } \\
\text { nach der }\end{array}$} & \multirow[b]{2}{*}{ 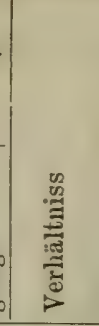 } & \multirow[b]{2}{*}{ 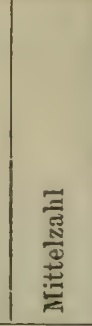 } & \multirow[b]{2}{*}{ Anmerkung } \\
\hline & & & & & 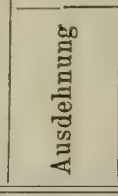 & 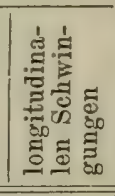 & & & 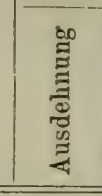 & 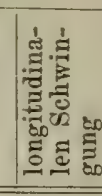 & & & \\
\hline Pappel & . & $64(1)$ & $\begin{array}{r}\text { N. }[2] \\
{[3]}\end{array}$ & 22.55 & $\begin{array}{l}535.1 \\
612.5\end{array}$ & $\begin{array}{l}496.6 \\
580.2\end{array}$ & $\begin{array}{l}0.928 \\
0.947\end{array}$ & 0.937 & $\begin{array}{l}12.00 \\
12.52\end{array}$ & $\begin{array}{l}11.56 \\
12.19\end{array}$ & $\begin{array}{l}0.963 \\
0.974\end{array}$ & 0.968 & \\
\hline Pappel & . . & $64(3)$ & N. [4] & 22.55 & 736.6 & 765.3 & 1.039 & 1.039 & 13.16 & 13.41 & 1.019 & 1.019 & \\
\hline Alkazie & . & $66(1)$ & $\begin{array}{r}\text { O. }[2] \\
{[3]} \\
{[4]}\end{array}$ & 16.63 & $\begin{array}{l}1418.8 \\
1334.8 \\
1465.0\end{array}$ & $\begin{array}{l}1871.6 \\
1639.0 \\
1568.0\end{array}$ & $\begin{array}{l}1.319 \\
1.228 \\
1.070\end{array}$ & 1.206 & $\begin{array}{l}13.21 \\
13.52 \\
13.11\end{array}$ & $\begin{array}{l}15.18 \\
14.98 \\
13.57\end{array}$ & $\begin{array}{l}1.149 \\
1.108 \\
1.035\end{array}$ & 1.097 & \\
\hline Tanne & $\cdot \cdot$ & $18(1)$ & $\begin{array}{r}\text { O. }[2] \\
{[3]} \\
{[4]} \\
{[5]}\end{array}$ & 18.89 & $\begin{array}{r}909.2 \\
1154.1 \\
1056.1 \\
1473.5\end{array}$ & $\begin{array}{r}968.9 \\
1388.6 \\
1443.0 \\
1482.9\end{array}$ & $\begin{array}{l}1.065 \\
1.203 \\
1.366 \\
1.006\end{array}$ & 1.160 & $\begin{array}{l}14.04 \\
14.69 \\
13.67 \\
15.79\end{array}$ & $\begin{array}{l}14.49 \\
16.12 \\
15.98 \\
15.84\end{array}$ & $\begin{array}{l}1.032 \\
1.097 \\
1.169 \\
1.003\end{array}$ & 1.075 & \\
\hline Tanne & . . & $18(3)$ & O. $\begin{array}{r}{[2]} \\
{[5]}\end{array}$ & 17.97 & \begin{tabular}{|r|}
853.3 \\
1385.1
\end{tabular} & $\begin{array}{r}914.0 \\
1337.7\end{array}$ & $\begin{array}{l}1.071 \\
0.966\end{array}$ & 1.018 & $\left\{\begin{array}{l}14.18 \\
16.37\end{array}\right.$ & $\begin{array}{l}14.68 \\
16.09\end{array}$ & $\begin{array}{l}1.035 \\
0.983\end{array}$ & 1.009 & \\
\hline Tanne & . & $18(5)$ & O. $\left[\begin{array}{r}2] \\
{[3]} \\
{[4]}\end{array}\right.$ & 19.84 & $\begin{array}{r}826.1 \\
1177.6 \\
1328.2\end{array}$ & $\begin{array}{r}828.5 \\
1207.2 \\
1384.6\end{array}$ & $\begin{array}{l}1.003 \\
1.025 \\
1.042\end{array}$ & 1.023 & $\begin{array}{l}13.38 \\
14.86 \\
15.98\end{array}$ & $\begin{array}{l}13.40 \\
15.04 \\
16.32\end{array}$ & $\begin{array}{l}1.001 \\
1.012 \\
1.021\end{array}$ & 1.011 & \\
\hline Tanne & . . & $65(1)$ & $\begin{array}{l}{[2]} \\
{[3]} \\
{[4]} \\
{[5]}\end{array}$ & & $\begin{array}{r}613.8 \\
496.7 \\
1331.7 \\
1285.0\end{array}$ & $\begin{array}{r}664.8 \\
630.2 \\
1421.2 \\
1498.9\end{array}$ & $\begin{array}{l}1.083 \\
1.269 \\
1.067 \\
1.166\end{array}$ & & $\begin{array}{l}11.02 \\
11.09 \\
15.82 \\
15.48\end{array}$ & $\begin{array}{l}11.47 \\
12.49 \\
16.34 \\
16.71\end{array}$ & $\begin{array}{l}1.041 \\
1.126 \\
1.033 \\
1.079\end{array}$ & & \\
\hline & & $65(2)$ & $\begin{array}{l}\lfloor 2] \\
{[4]} \\
{[5]}\end{array}$ & & $\begin{array}{r}858.0 \\
1168.9 \\
1464.5\end{array}$ & $\begin{array}{r}923.1 \\
1188.9 \\
1555.6\end{array}$ & $\begin{array}{l}1.076 \\
1.017 \\
1.063\end{array}$ & & $\begin{array}{l}13.81 \\
15.49 \\
16.75\end{array}$ & $\begin{array}{l}14.32 \\
15.62 \\
17.26\end{array}$ & $\begin{array}{l}1.037 \\
1.008 \\
1.030\end{array}$ & & \\
\hline & & $65(3)$ & $\begin{array}{l}{[2]} \\
{[4]} \\
{[5]}\end{array}$ & & $\begin{array}{l}1096.0 \\
1378.6 \\
1335.0\end{array}$ & $\begin{array}{l}1053.0 \\
1276.7 \\
1364.7\end{array}$ & $\begin{array}{l}0.961 \\
0.926 \\
1.022\end{array} \mid$ & & $\begin{array}{l}1443 \\
16.28 \\
16.45\end{array}$ & $\begin{array}{l}14.14 \\
15.67 \\
16.62\end{array}$ & $=\begin{array}{l}0.979 \\
0.962 \\
1.010\end{array}$ & & \\
\hline & & $65(4)$ & $\begin{array}{l}{[3]} \\
{[4]} \\
{[5]}\end{array}$ & 10.26 & $\begin{array}{l}1085.7 \\
1365.2 \\
1290.5\end{array}$ & \begin{tabular}{|l|}
1167.1 \\
1413.2 \\
1446.6
\end{tabular} & $\begin{array}{l}1.075 \\
1.035 \\
1.121\end{array}$ & 1.051 & $\begin{array}{l}14.34 \\
15.25 \\
15.28\end{array}$ & $\begin{array}{l}14.87 \\
15.51 \\
16.18\end{array}$ & $\begin{array}{l}1.037 \\
1.017 \\
1.059\end{array}$ & 1.024 & \\
\hline & & $65(5)$ & $\begin{array}{l}{[2]} \\
{[3]} \\
4]\end{array}$ & & \begin{tabular}{|}
858.1 \\
1053.2 \\
1056.8
\end{tabular} & $\begin{array}{r}941.2 \\
972.1 \\
1016.2\end{array}$ & $\begin{array}{l}1.097 \\
0.923 \\
0.961\end{array}$ & & $\left|\begin{array}{l}13.12 \\
14.82 \\
14.25\end{array}\right|$ & $\begin{array}{l}13.74 \\
14.24 \\
13.98\end{array}$ & $\begin{array}{l}1.047 \\
0.961 \\
0.981\end{array} \mid$ & & \\
\hline
\end{tabular}




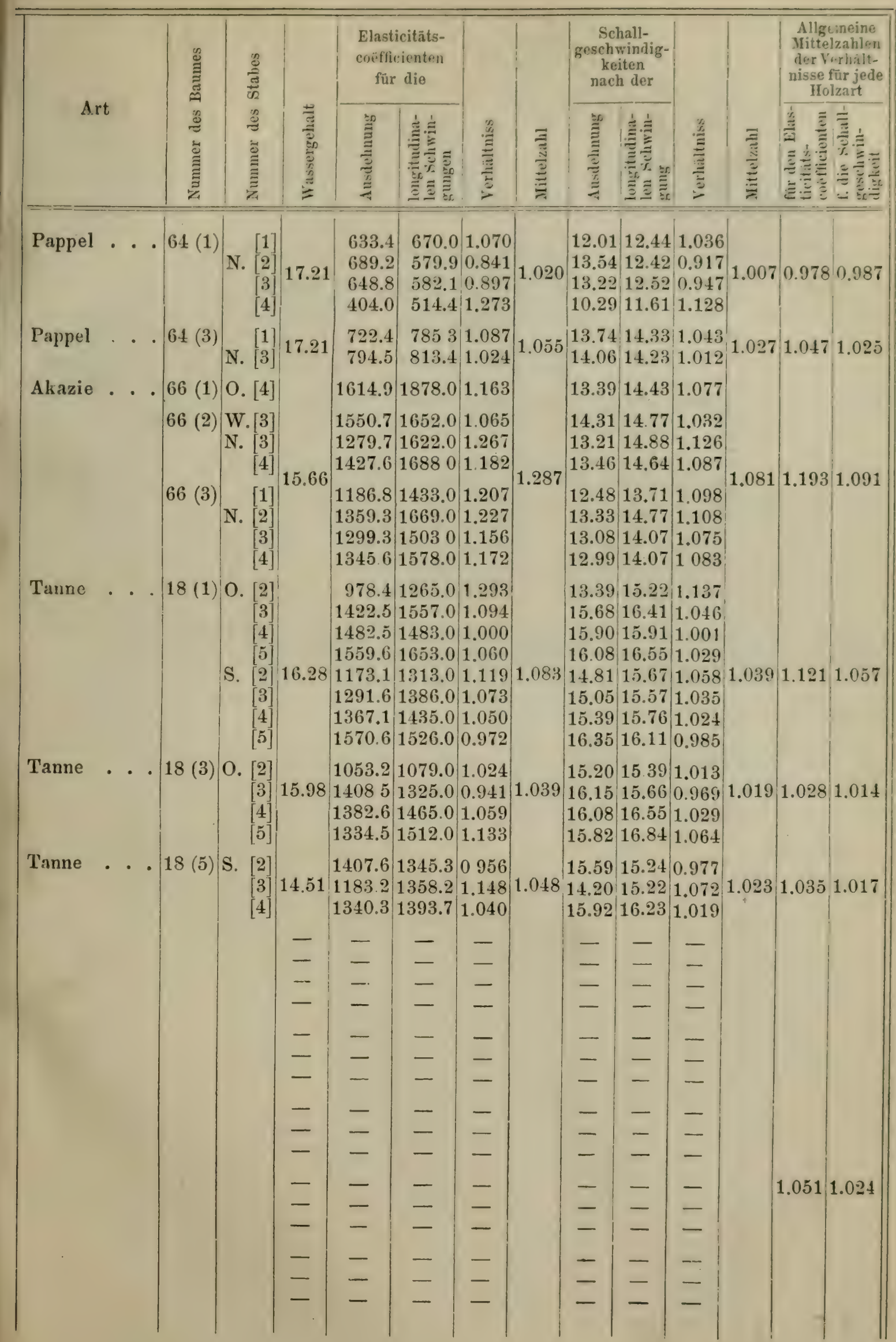




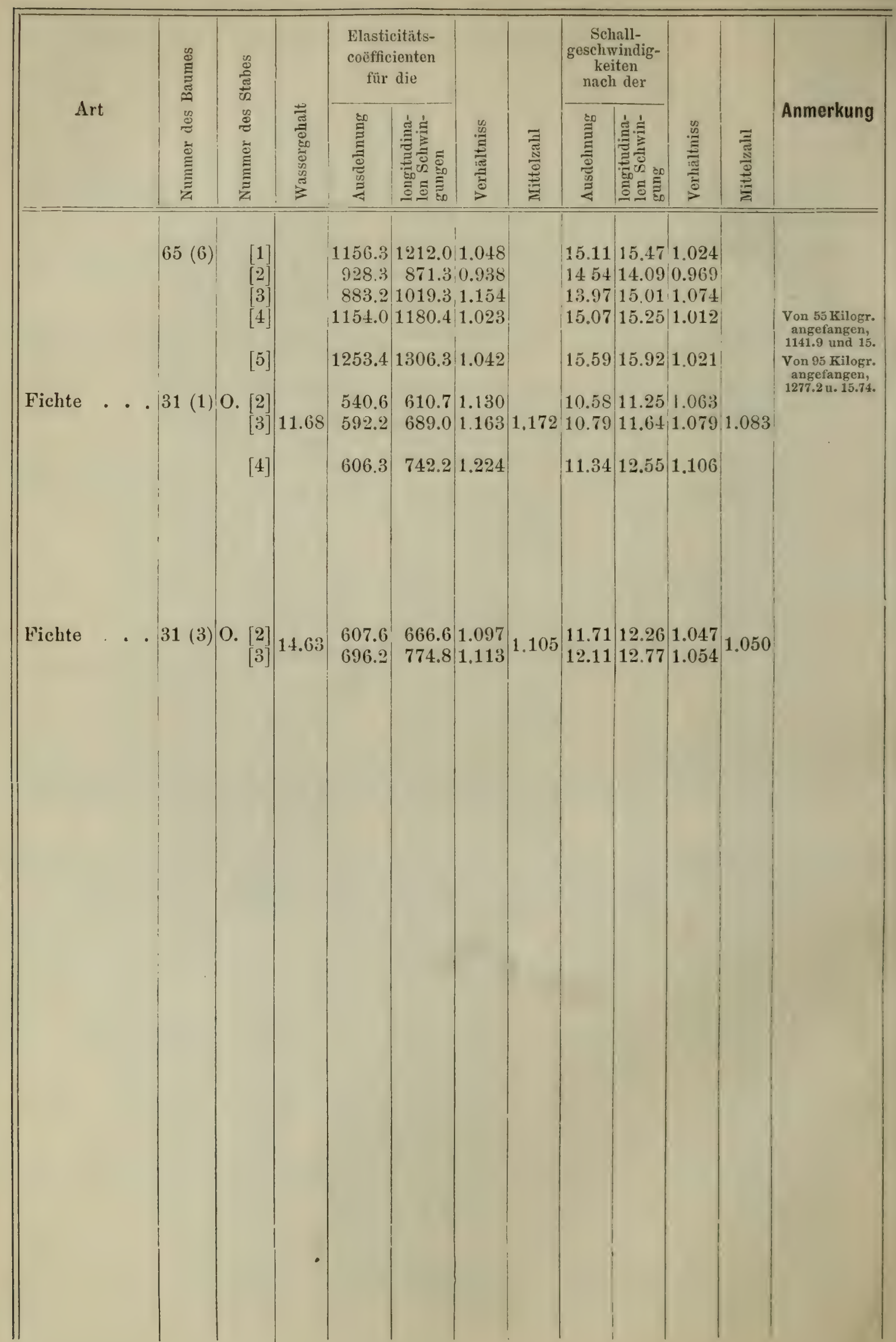




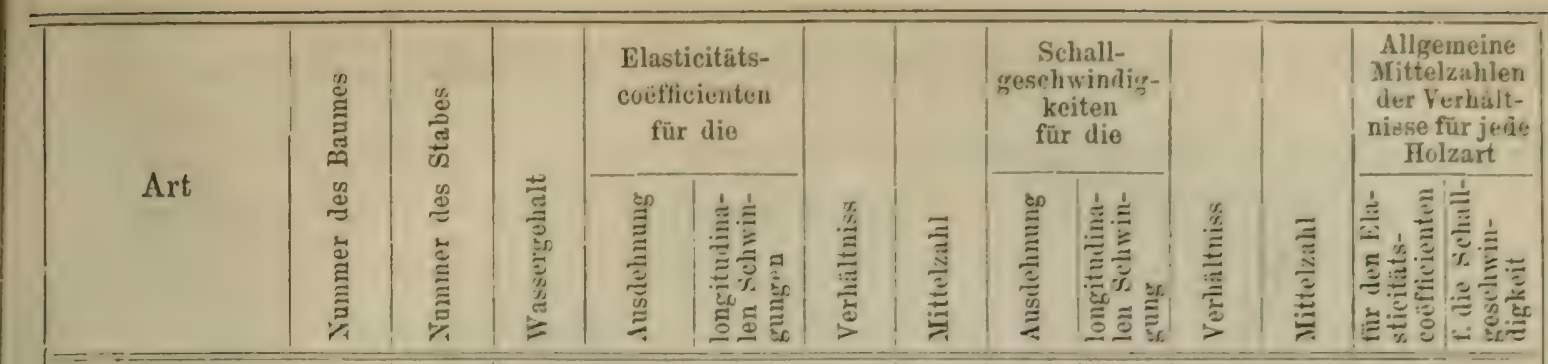

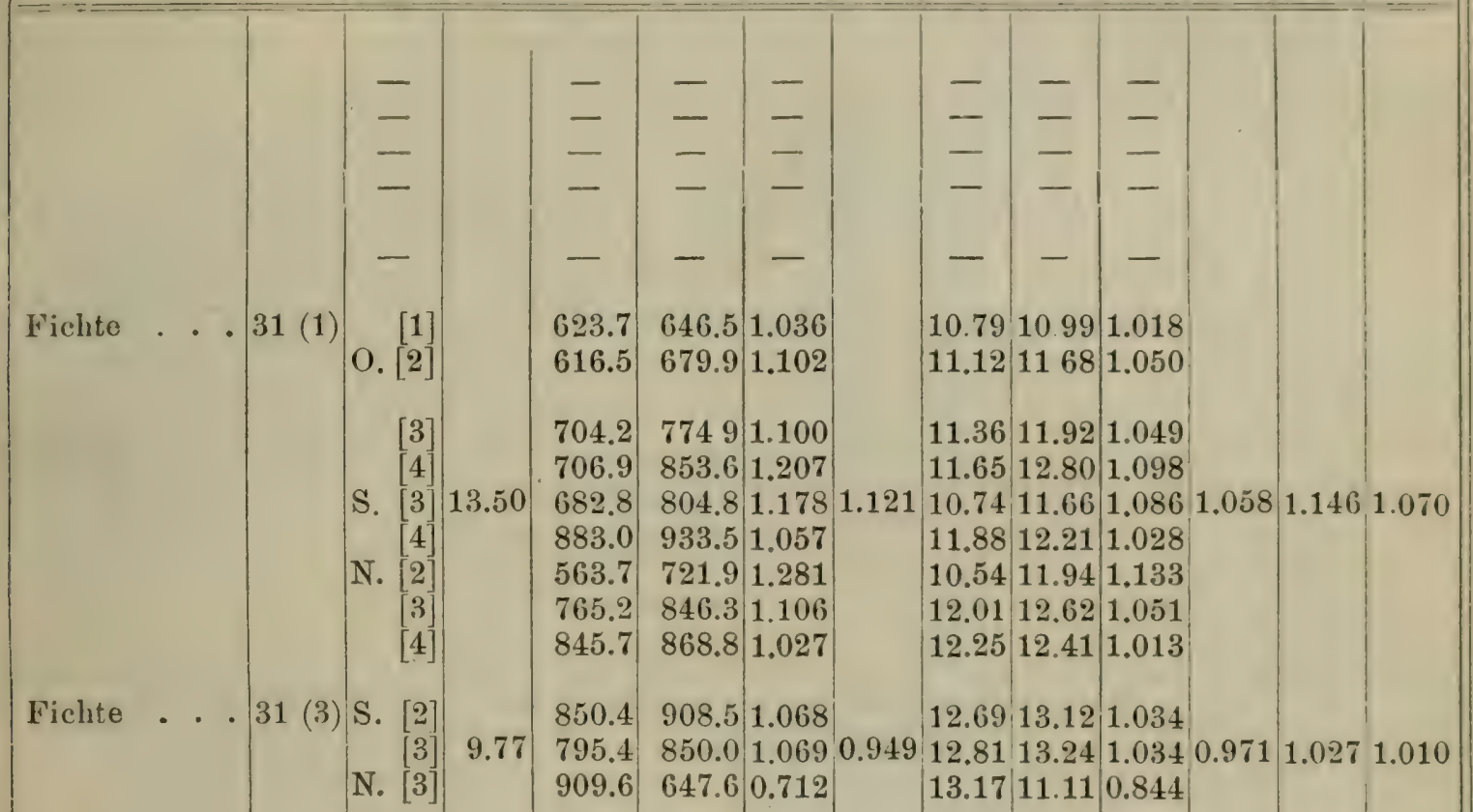


Tabelle

Specifisches Gewicht, Schallgeschwindigkeit, Elasticitätscoëfficient, Fesnach den vier

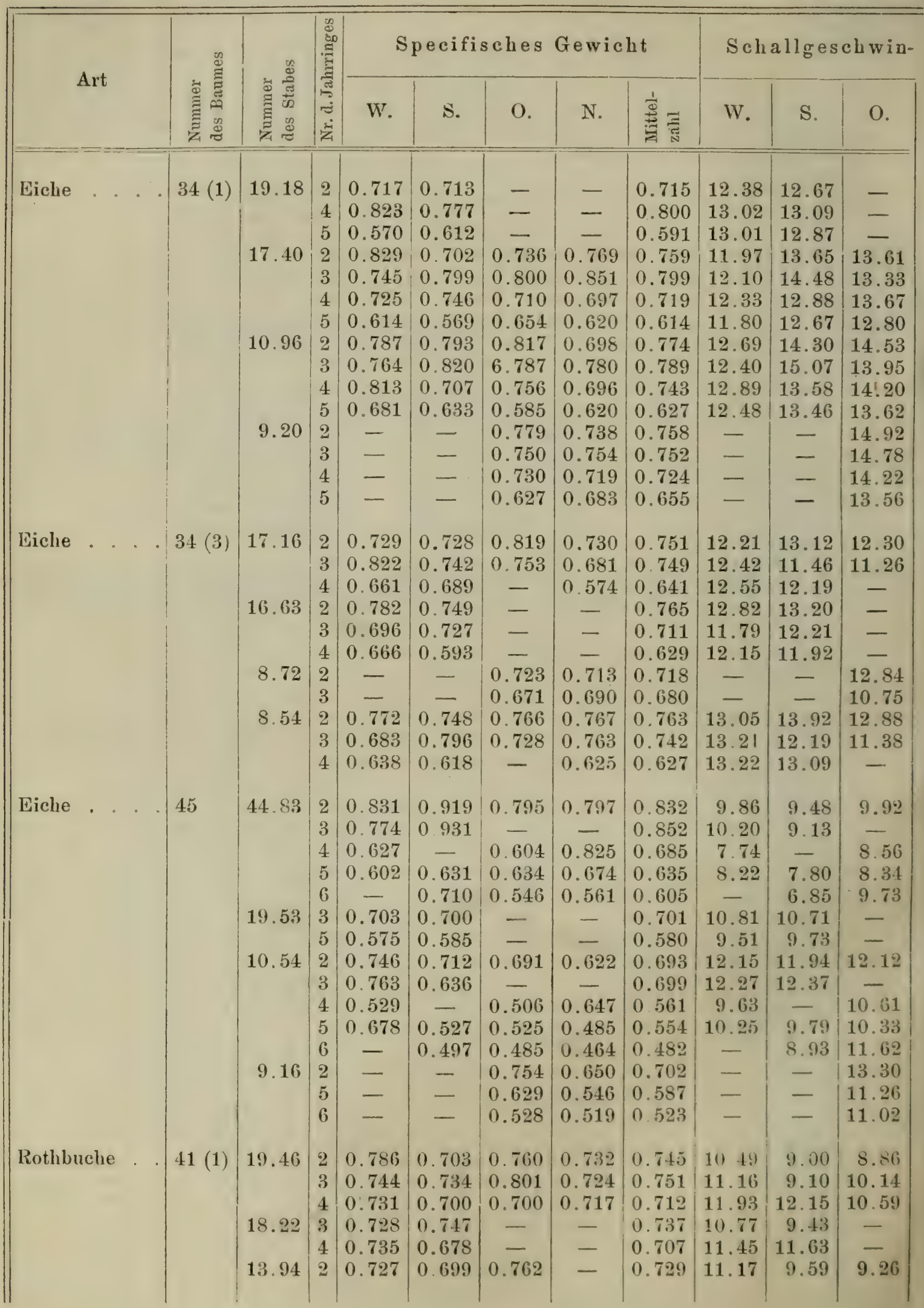


Nr. VIII.

tigkeit im Sinne der Fasern in ein und denselben Jahrringen und Weltgegenden.

digkeit

N. 产
Elasticitätscoëfficient

O.
S.

Festigkeit in $\square$ Millim.

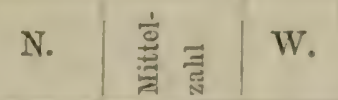

S. $\quad 0$.

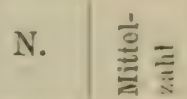

\begin{tabular}{|c|c|c|c|c|c|c|c|c|c|c|c|}
\hline - & 12.52 & 1236.8 & 1288.8 & 一 & - & 1262.8 & 2.90 & 7.22 & - & - & 5.06 \\
\hline - & 13.05 & 1570.8 & 1497.4 & - & 一 & 1534.1 & 9.67 & 8.15 & - & - & 8.91 \\
\hline- & 12.94 & 1086.9 & 1141.3 & - & - & 1114.1 & 5.41 & 7.54 & 一 & - & 6.47 \\
\hline 13.61 & 13.21 & 1346.7 & 1472.0 & 1534.2 & 1602.5 & 1488.8 & - & - & - & - & - \\
\hline 12.30 & 13.05 & 1227.5 & 1885.3 & 1599.7 & 1448.9 & 1540.3 & - & - & - & - & - \\
\hline 11.50 & 12.59 & 1240.4 & 1394.4 & 1493.1 & 1039.0 & 1291.7 & - & - & - & - & - \\
\hline i1. 37 & 12.16 & 1074.6 & 1023.6 & 1205.9 & 9020 & 1051.5 & - & - & - & - & - \\
\hline 14.40 & 13.98 & 1425.8 & 1825.5 & 1940.8 & $\mid 1628.3$ & 1705.1 & 2.20 & 5.29 & 9.69 & 3.85 & 5.26 \\
\hline 12.96 & 13.59 & 1321.5 & 2096.1 & 1723.4 & 1475.2 & 1654.0 & 6.62 & 9.69 & 7.05 & 3.52 & 6.72 \\
\hline 12.16 & 13.21 & 1520.0 & 1466.8 & 1714.6 & 1188.3 & 1472.4 & 7.53 & 9.03 & 11.45 & 7.49 & 8.87 \\
\hline 11.96 & 12.88 & 1193.9 & 1291.2 & 1220.8 & 998.3 & 1176.0 & 8.39 & 9.03 & 7.49 & 5.85 & 7.69 \\
\hline 1305 & 13.98 & - & - & 1950.5 & 1411.6 & 1681.0 & - & - & - & - & - \\
\hline 12.03 & 13.40 & - & - & 1843.0 & 1228.2 & 1535.6 & - & - & - & - & - \\
\hline 14.00 & 14.11 & 一 & - & 1660.3 & 1588.3 & 1624.3 & - & - & - & - & - \\
\hline 12.18 & 12.87 & 一 & - & 1296.8 & 1139.1 & 1217.9 & - & 一 & - & - & - \\
\hline 12.24 & 12.47 & 1223.1 & 1410.3 & 1394.4 & 1230.8 & 13146 & - & -. & - & - & - \\
\hline 12.76 & 11.97 & 1438.2 & 1096.6 & 1074.3 & 1247.8 & 1214.2 & - & - & - & 一 & - \\
\hline 12.11 & 12.28 & 1171.6 & 1152.2 & 一 & 947.3 & 1090.4 & - & - & - & - & - \\
\hline - & 13.01 & 1445.7 & 1468.1 & - & - & 1456.9 & 6.45 & 5.00 & - & - & 5.72 \\
\hline - & 12.00 & 1088.2 & 1218.9 & - & - & 1153.5 & 5.32 & 7.34 & - & - & 6.33 \\
\hline- & 12.03 & 1106.8 & 948.9 & - & - & 1027.8 & 5.67 & 4.33 & - & - & 5.00 \\
\hline 13.18 & 13.01 & - & - & 1342.5 & 1395.3 & 1368.9 & - & - & - & - & - \\
\hline 12.01 & 11.38 & - & - & 1356.2 & 1121.1 & $12:$ & - & - & $=8$ & 5 & 50 \\
\hline 12.78 & 13.16 & 1478.7 & 1631.2 & 1430.6 & 1410.1 & 1487.6 & 9.43 & 716 & 5.8 & 5.3 & 6.94 \\
\hline 13.59 & 12.59 & 1341.3 & 1331.1 & 1060.3 & 1586.1 & 1329.7 & 5.70 & 5.37 & 9.40 & 6.27 & 6.68 \\
\hline 12.91 & 13.07 & 1255.5 & 1191.3 & - & 1171.6 & 1206.1 & 4.50 & 7.61 & - & 5.82 & 5.98 \\
\hline 9.40 & 9.66 & 9090 & 928.9 & 881.0 & 792.3 & 877.8 & - & - & - & - & - \\
\hline - & 9.66 & 905.7 & 873.2 & 一 & - & 889.4 & - & - & - & - & - \\
\hline 9.32 & 8.54 & 422.7 & - & 498.6 & 805.6 & 575.6 & - & - & - & - & - \\
\hline 7.37 & 7.93 & 458.1 & 430.9 & 495.8 & 412.3 & 449.3 & - & - & - & - & - \\
\hline 6.80 & 7.79 & - & 375.4 & 581.5 & 292.1 & $\$ 16.3$ & - & - & - & - & - \\
\hline - & 10.76 & 924.2 & 903.6 & - & - & 913.9 & 6.72 & 5.04 & - & - & 5.88 \\
\hline- & 9.62 & 585.0 & 623.3 & - & - & 604.1 & 2.98 & 4.62 & $=$ & - & 3.80 \\
\hline 12.14 & 12.09 & 1239.0 & 1141.0 & 1141.0 & 1031.0 & 1138.0 & 3.05 & 4.21 & 5.77 & 3.99 & $\begin{array}{l}4.25 \\
3.83\end{array}$ \\
\hline 15 & 12.32 & 1292.0 & 1095.0 & $\overline{11}$ & $\overline{0}$ & 1193.0 & 4.52 & 3.14 & - & - & $\begin{array}{l}3.85 \\
3.86\end{array}$ \\
\hline 9.81 & 10.04 & 801.0 & $5 \overline{69} 0$ & 630.0 & 526.0 & 631.0 & $\begin{array}{r}2.20 \\
4.11\end{array}$ & 35.5 & $\begin{array}{l}3.14 \\
3.11\end{array}$ & 3.14 & $\begin{array}{l}3.00 \\
334\end{array}$ \\
\hline 8.70 & 9.75 & - & 446.0 & 737.0 & 395.0 & 526.0 & - & 2.66 & 3.10 & 1.72 & 2.49 \\
\hline 12.95 & 13.12 & 一 & 一 & 1501.6 & 1227.0 & 1364.3 & - & - & - & - & - \\
\hline 10.43 & 10.84 & - & - & 898.0 & 668.0 & 783.0 & - & - & - & - & - \\
\hline 9.16 & 10.09 & $一$ & - & 722.2 & 490.0 & 606.1 & - & 一 & - & - & - \\
\hline 8.17 & 9.13 & 973.3 & $6+0.8$ & 671.4 & 543.1 & 707.1 & - & - & - & - & - \\
\hline 12.08 & 10.62 & 1042.8 & 684.0 & 926.8 & 1188.9 & 960.6 & - & - & - & - & - \\
\hline 11.47 & 11.53 & 1162.6 & 1162.9 & 885.7 & 1060.6 & 1067.9 & - & - & - & - & - \\
\hline- & 10.10 & 950.6 & 747.6 & - & 826.5 & 841.6 & 6.91 & 5.89 & 一 & - & 6.40 \\
\hline & 11.54 & 1083.7 & 1032.2 & - & 1057.9 & 1057.9 & 3.48 & 5.81 & - & - & 4.64 \\
\hline 8.58 & 9.65 & 1020.5 & 723.7 & 735.3 & - & 826.5 & 5.79 & 5.12 & 4.27 & 4. 27 & 4.86 \\
\hline
\end{tabular}




\section{$-\quad 84$}

\begin{tabular}{|c|c|c|c|c|c|c|c|c|c|c|c|}
\hline \multirow{2}{*}{ Art } & \multirow{2}{*}{ 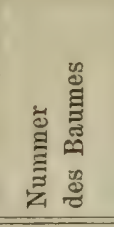 } & \multirow{2}{*}{ 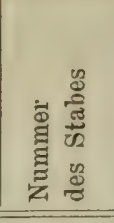 } & \multirow{2}{*}{ 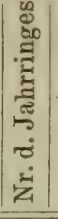 } & \multicolumn{3}{|c|}{ Specifisches } & \multicolumn{2}{|c|}{ Gewicht } & \multicolumn{3}{|c|}{ Schallgeschwin- } \\
\hline & & & & W. & s. & o. & $\mathrm{N}$. & 暯 & W. & S. & o. \\
\hline Rothbuche & & 9.77 & $\begin{array}{l}3 \\
4 \\
2 \\
4\end{array}$ & $\begin{array}{c}0.715 \\
0.704 \\
-\end{array}$ & $\begin{array}{c}0.738 \\
0.705 \\
- \\
-\end{array}$ & $\begin{array}{l}0.754 \\
0.734 \\
0 . \overline{727}\end{array}$ & $\begin{array}{l}0.654 \\
0.691 \\
0.779 \\
0.692\end{array}$ & $\begin{array}{l}0.716 \\
0.708 \\
0 \\
0.779 \\
0.709\end{array}$ & $\begin{array}{c}11.53 \\
12.44 \\
=\end{array}$ & $\begin{array}{c}10.26 \\
12.91 \\
-\end{array}$ & $\begin{array}{r}10.56 \\
11.47 \\
8.83 \\
11.28\end{array}$ \\
\hline Rothbuche & $41(3)$ & $\begin{array}{r}16.50 \\
15.69 \\
11.83 \\
7.63\end{array}$ & $\begin{array}{l}3 \\
2 \\
3 \\
2 \\
3 \\
2 \\
3\end{array}$ & $\begin{array}{c}0.752 \\
0 . \overline{7} 48 \\
0 . \overline{7} 86 \\
- \\
-\end{array}$ & $\begin{array}{l}0.769 \\
0.784 \\
0.688 \\
0.747 \\
0.743 \\
- \\
-\end{array}$ & $\begin{array}{c}0 . \overline{780} \\
0.721 \\
0.762 \\
0.757 \\
0.768 \\
0.715\end{array}$ & $\begin{array}{l}\overline{-} \\
0.831 \\
0.677 \\
0.816 \\
0.709 \\
0.796 \\
0.677\end{array}$ & $\begin{array}{l}0.760 \\
0.798 \\
0.708 \\
0.775 \\
0.744 \\
0.782 \\
0.696\end{array}$ & $\begin{array}{c}9.72 \\
- \\
1100 \\
- \\
11.46 \\
-\end{array}$ & $\begin{array}{r}10.42 \\
9.90 \\
11.91 \\
10.07 \\
12.04 \\
- \\
-\end{array}$ & $\begin{array}{c}- \\
10.20 \\
11.17 \\
10.67 \\
11.40 \\
10.46 \\
10.50\end{array}$ \\
\hline Rothbuche & 46 & $\begin{array}{r}22.41 \\
\\
13.92 \\
11.49 \\
9.52\end{array}$ & $\begin{array}{l}2 \\
3 \\
4 \\
4 \\
2 \\
3 \\
4 \\
3 \\
4\end{array}$ & $\begin{array}{c}0.768 \\
0 . \\
0.686 \\
0.674 \\
0.729 \\
0.703 \\
0.664 \\
- \\
-\end{array}$ & $\begin{array}{c}0 . \overline{717} \\
0.668 \\
0.669 \\
\overline{-} \\
0.790 \\
0.741 \\
- \\
-\end{array}$ & $\begin{array}{c}\overline{-} \\
0.683 \\
0.679 \\
- \\
\overline{-} \\
0.749 \\
0.648 \\
0.709 \\
0.658\end{array}$ & $\begin{array}{l}0.800 \\
0.686 \\
0.657 \\
- \\
0.767 \\
0.770 \\
0.713 \\
0.674 \\
0.700\end{array}$ & $\begin{array}{l}0.784 \\
0.695 \\
0.672 \\
0.671 \\
0.748 \\
0753 \\
0.691 \\
0.691 \\
0.679\end{array}$ & $\begin{array}{c}10.58 \\
10.99 \\
10.17 \\
12.65 \\
13.57 \\
13.11 \\
12.64 \\
=\end{array}$ & $\begin{array}{c}-- \\
950 \\
9.59 \\
11.98 \\
- \\
11.00 \\
11.88 \\
- \\
-\end{array}$ & $\begin{array}{c}- \\
10.10 \\
10.28 \\
- \\
- \\
12.41 \\
12.76 \\
11.81 \\
11.92\end{array}$ \\
\hline Akazie & $66(1)$ & $\begin{array}{r}18.43 \\
16 \quad 63 \\
15.66\end{array}$ & \begin{tabular}{|l}
3 \\
4 \\
3 \\
4 \\
3 \\
4
\end{tabular} & $\begin{array}{l}0.738 \\
0.790 \\
0.649 \\
0.757 \\
0.698 \\
0.801\end{array}$ & $\begin{array}{l}0.668 \\
0.796 \\
0.647 \\
0.706 \\
0.665 \\
0.729\end{array}$ & $\begin{array}{c}0.762 \\
0.822 \\
- \\
\overline{-} \\
0.711 \\
0.730\end{array}$ & $\begin{array}{l}= \\
= \\
= \\
=\end{array}$ & $\begin{array}{l}0.723 \\
0.803 \\
0.648 \\
0.731 \\
0.691 \\
0.753\end{array}$ & $\begin{array}{l}14.21 \\
13.99 \\
14.98 \\
13.57 \\
14.79 \\
14.43\end{array}$ & $\begin{array}{l}14.77 \\
14.53 \\
13.85 \\
13.79 \\
14.53 \\
14.77\end{array}$ & $\begin{array}{c}14.22 \\
12.65 \\
- \\
14.29 \\
12.66\end{array}$ \\
\hline Akazi & $66(2)$ & $\begin{array}{r}18.43 \\
1566 \\
9.82\end{array}$ & $\begin{array}{l}3 \\
4 \\
3 \\
4 \\
3 \\
4\end{array}$ & $\begin{array}{l}\bar{z} \\
\overline{-} \\
\overline{-}\end{array}$ & $\begin{array}{c}- \\
\overline{-} \\
\overline{-} \\
\overline{7} \\
-\end{array}$ & $\begin{array}{l}0.695 \\
0.719 \\
0.673 \\
0.709 \\
0.678 \\
0.711\end{array}$ & $\begin{array}{l}0.654 \\
0.671 \\
0.651 \\
0.700 \\
0.630 \\
0.736\end{array}$ & $\begin{array}{l}0.674 \\
0.695 \\
0.662 \\
0.722 \\
0.654 \\
0.723\end{array}$ & $\begin{array}{l}\bar{z} \\
\bar{z} \\
\overline{-}\end{array}$ & $\begin{array}{c}- \\
13.63 \\
- \\
13.85 \\
-\end{array}$ & $\begin{array}{l}14.67 \\
13.63 \\
14.77 \\
13.63 \\
15.52 \\
15.64\end{array}$ \\
\hline Akazie & $66(4)$ & $\begin{array}{l}18: 43 \\
15.66\end{array}$ & $\begin{array}{l}4 \\
4\end{array}$ & - & - & $\begin{array}{l}0.704 \\
0.718\end{array}$ & $\begin{array}{l}0.754 \\
0.734\end{array}$ & $\begin{array}{l}0.729 \\
0.726\end{array}$ & - & $\overline{-}$ & 14.77 \\
\hline Tanne & $18(1)$ & 16.28 & $\begin{array}{l}2 \\
3 \\
4 \\
5 \\
2 \\
3\end{array}$ & $\begin{array}{c}0.424 \\
0.462 \\
0.494 \\
0.508 \\
0.410 \\
0.475 \\
0.502 \\
0.525 \\
0.485 \\
05514 \\
0.521 \\
0.536 \\
- \\
-\end{array}$ & $\begin{array}{l}0.421 \\
0.432 \\
0.468 \\
0.512 \\
0.403 \\
0.435 \\
0.464 \\
0.556 \\
0.475 \\
0.508 \\
0.513 \\
0.522 \\
-\end{array}$ & $\begin{array}{c}0.395 \\
0.561 \\
0586 \\
0.521 \\
- \\
- \\
- \\
0.434 \\
0.539 \\
0.572 \\
0.517 \\
0.540 \\
0.526\end{array}$ & $\begin{array}{l}0.380 \\
0.545 \\
0.438 \\
0.493 \\
- \\
- \\
\overline{-} \\
0.402 \\
0.481 \\
0.461 \\
0.529 \\
0.544 \\
0.500\end{array}$ & $\begin{array}{l}0.405 \\
0.500 \\
0.496 \\
0.508 \\
0.406 \\
0.455 \\
0.483 \\
0.540 \\
0.449 \\
0.510 \\
0.517 \\
0.526 \\
0.542 \\
0.513\end{array}$ & $\begin{array}{c}14.01 \\
15.72 \\
15.11 \\
15.85 \\
14.49 \\
16.12 \\
15.98 \\
15.84 \\
15.22 \\
16.41 \\
15.91 \\
16.55 \\
- \\
-\end{array}$ & $\begin{array}{c}14.80 \\
14.81 \\
14.98 \\
15.29 \\
15.15 \\
15.47 \\
14.01 \\
15.11 \\
15.67 \\
15.57 \\
15.76 \\
16.11 \\
- \\
-\end{array}$ & $\begin{array}{c}14.91 \\
14.69 \\
11.73 \\
15.80 \\
= \\
- \\
- \\
15.69 \\
15.35 \\
1232 \\
16.32 \\
12.99 \\
16.93\end{array}$ \\
\hline
\end{tabular}




\begin{tabular}{|c|c|c|c|c|c|c|c|c|c|c|c|}
\hline \multicolumn{2}{|c|}{ digkeit } & \multicolumn{5}{|c|}{ Elasticitätscoëfficient } & \multicolumn{5}{|c|}{ Festigkeit in $\square$ Millim. } \\
\hline N. & $\stackrel{\frac{1}{\Xi}}{\Xi}$ & W. & S. & O. & N. & $\stackrel{i}{\Xi} \equiv$ & IV. & $\therefore$. & O. & N. & $\stackrel{\frac{1}{3}}{\equiv}$ \\
\hline 12.52 & 11.22 & 1070.1 & 874.8 & 947.1 & 1161.3 & 1013.3 & 7.75 & 5.12 & 7. 26 & 3.42 & 5.89 \\
\hline 12.01 & 12.21 & 12258 & 1321.5 & 1087.1 & 1120.8 & 1185.8 & 6.52 & t. $7(0)$ & 7.31 & 555 & (6.) 02 \\
\hline 8.64 & 8.73 & - & - & -- & $65+1.1$ & 654.1 & - & - & 一 & - & - \\
\hline 12.47 & 11.87 & - & - & 1041.4 & 1210.3 & 1125.8 & - & - & - & - & - \\
\hline - & 10.07 & 7990 & 939.4 & - & 一 & 869.2 & 2. 41 & 5.87 & - & - & $+\frac{14}{4}$ \\
\hline 11.33 & 10.48 & - & 864.7 & 913.2 & 1200.5 & 992.8 & - & - & - & - & - \\
\hline 12.30 & 11.59 & 1018.4 & 1098.2 & 1010.9 & 1152.7 & 1070.0 & 一 & - & - & - & - \\
\hline 11.56 & 10.76 & - & 852.6 & 9767 & 1226.7 & 1018.7 & - & 4.8 .3 & t. $8: 3$ & 5. 93 & 5. 19 \\
\hline 12.70 & 11.90 & 1161.0 & 1213.0 & 1106.4 & 1287.1 & 1191.9 & 6.01 & $5 \quad 49$ & $7+6$ & 7.32 & 6.57 \\
\hline 11. 23 & 10.84 & - & - & 946.4 & 1130.2 & 1038.3 & - & - & - & - & - \\
\hline 13.05 & 11.77 & - & - & 8874 & 1297.4 & 1092.4 & - & - & - & - & - \\
\hline 10.28 & 10.43 & 967.3 & - & - & 951.4 & 959.3 & - & - & - & - & - \\
\hline อ. 43 & 10.00 & - & 728.2 & 784.0 & 6865 & 732.9 & - & - & - & - & - \\
\hline 9.71 & 9.94 & 798.4 & 691.3 & 807.1 & 698.5 & $7 \pm 8.8$ & $\ldots$ & - & $\ldots$ & - & - \\
\hline- & 12.31 & 1214.1 & 1080.0 & - & - & $11+7.0$ & 4.28 & 286 & - & - & 3.57 \\
\hline 12.33 & 12.95 & 1510.7 & - & - & 1311.9 & 1411.3 & 8.33 & - & - & 8.08 & 8.20 \\
\hline 11.95 & 12.12 & 1359.8 & 1075.0 & 1298.0 & 1237.9 & $12+2.7$ & 4.58 & $4(1) 4$ & 808 & 4.94 & 5. 41 \\
\hline 11.94 & 12.30 & 1193.9 & 1177.4 & 1187.5 & 1144 ว & 1175.8 & $4.7 t$ & อั 39 & 6.29 & t. 04 & 5 11 \\
\hline 11.33 & 11.57 & - & - & 1113.7 & 973.0 & 1043.3 & - & - & - & - & - \\
\hline 10.39 & 11.15 & - & - & 1052.8 & 851.1 & 951.9 & - & - & - & - & - \\
\hline- & 14.40 & 1679.0 & 1640.0 & 1734.0 & - & $168+.3$ & - & - & - & - & - \\
\hline- & 13.72 & 17.40 .0 & 1889.0 & 1480.0 & - & 1703.0 & - & - & - & - & - \\
\hline- & 14.41 & 1639.0 & 1396.7 & - & - & 1517.8 & 6.26 & 11.59 & - & - & 8.92 \\
\hline$\ldots$ & 13.68 & 1568.8 & 1510.9 & - & - & 1539.8 & 9.25 & 9.06 & 一 & 一 & 9.15 \\
\hline- & 14.54 & 1719.1 & 1579.1 & 1634.0 & - & $16+4.1$ & 8.49 & 13.58 & - & - & 11.03 \\
\hline- & 13.95 & 1878.0 & 1789.3 & 1316.0 & - & 1661.1 & 7.20 & 11.45 & 10.21 & - & 9.62 \\
\hline 14.70 & 14.67 & - & - & 1676.0 & 1592.0 & $163 \pm .0$ & - & - & - & - & - \\
\hline 14.40 & 13.89 & - & 1681.0 & 15040 & 1565.0 & 1583.3 & - & - & - & - & - \\
\hline 14.88 & 14.82 & - & - & 1652.0 & 1622.0 & 1637.0 & - & - & - & 10.30 & 10.30 \\
\hline 14.64 & 14.04 & - & 16330 & 1483.0 & 1688.0 & 1601.3 & - & $11.0 \pm$ & 8. 66 & - & 9.85 \\
\hline 16.33 & 15.92 & - & - & 1984.0 & 1790.0 & 1887.0 & - & - & - & - & - \\
\hline 15.89 & 15.76 & - & - & 2161.0 & 19955.0 & 2078.0 & - & - & - & - & - \\
\hline 14.18 & 14.47 & - & - & 1728.0 & 1706.0 & 1717.0 & - & - & - & - & - \\
\hline 14.53 & 14.65 & - & - & 1762.0 & 1743.0 & 1752.5 & - & - & 10.19 & 1358 & 11.88 \\
\hline 14.58 & 14.57 & 936.5 & 1037.6 & 988.1 & 909.0 & 967.8 & - & - & - & - & - \\
\hline 17.04 & 15.54 & 1283.2 & $106+.9$ & 13439 & 1780.9 & 1368.2 & - & - & - & - & - \\
\hline 15.31 & 14.28 & 1269.2 & 1181.8 & 1062.1 & 1155.3 & 1167.1 & - & - & $\ldots$ & - & - \\
\hline 16.12 & 15.76 & 1436.2 & 1347.0 & 1463.7 & $14 t 1.7$ & $1+2: 2.1$ & - & -- & - & - & - \\
\hline- & 14.82 & 968.9 & $10 \leftarrow 0.9$ & - & - & $100+.9$ & 4.32 & t. 11 & - & - & 4.21 \\
\hline- & 15.79 & 1388.6 & 1171.8 & - & - & 1280.2 & 4.88 & 4.92 & - & - & \pm .90 \\
\hline- & 14.99 & 1443.0 & 1024.3 & - & - & 1233.6 & $\begin{array}{r}4.30 \\
\end{array}$ & 6.34 & - & - & 5.32 \\
\hline - & 15.47 & 1482.9 & 1428.5 & - & - & 1455.7 & 5.81 & 5.42 & - & - & 5.61 \\
\hline 15.33 & 15.48 & 1265.0 & 1313.0 & 1202.6 & 1063.4 & 12110 & 4.23 & 3.93 & +.89 & 4.68 & 4.43 \\
\hline 17.86 & 16.29 & 1557.0 & 1386.0 & 1496.0 & 1726.6 & $15+1.4$ & 4.22 & 3.77 & 4.68 & ว. 96 & 4.66 \\
\hline 16.13 & 15.03 & 1483.0 & 1435.0 & 976.3 & 1350.0 & 1311.1 & 4.71 & 5. 39 & 3.83 & 5.53 & 4.87 \\
\hline 16.85 & 16.46 & 1653.0 & 1526.0 & 1550.2 & 1689.7 & 1604.7 & 6.42 & 11.40 & 8.72 & 7.23 & 8.44 \\
\hline 16.63 & 14.81 & - & - & 1025.5 & 1693.8 & 13559.6 & - & - & - & - & - \\
\hline 17.09 & 17.01 & - & - & 1697.7 & 1642.5 & 1670.1 & - & - & - & - & - \\
\hline
\end{tabular}




\section{$-\quad 86$}

\begin{tabular}{|c|c|c|c|c|c|c|c|c|c|c|c|c|}
\hline \multirow{2}{*}{\multicolumn{2}{|c|}{ Art }} & \multirow{2}{*}{ 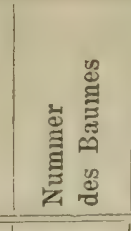 } & \multirow{2}{*}{ 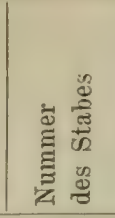 } & \multirow{2}{*}{ 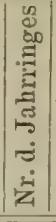 } & \multicolumn{5}{|c|}{ Specifisches Gewicht } & \multicolumn{3}{|c|}{ Schallgeschwin. } \\
\hline & & & & & W. & S. & O. & N. & $\begin{array}{l}\frac{1}{c} \\
\text { 总 }\end{array}$ & W. & S. & $\mathrm{O}$. \\
\hline Tanne & . & $18(3)$ & $\begin{array}{l}18.65 \\
17.97\end{array}$ & $\begin{array}{l}2 \\
3 \\
4 \\
5 \\
2 \\
3 \\
4 \\
5 \\
2 \\
3 \\
4 \\
5 \\
2 \\
4 \\
5\end{array}$ & $\begin{array}{c}0.407 \\
0.462 \\
0.447 \\
0.458 \\
0.377 \\
0.474 \\
0.500 \\
0.459 \\
0.405 \\
0.480 \\
0.475 \\
0.474 \\
- \\
-\end{array}$ & $\begin{array}{c}0.520 \\
0.514 \\
0.473 \\
0.468 \\
0.394 \\
0.480 \\
0.509 \\
0.425 \\
0.532 \\
0.459 \\
0.547 \\
0.494 \\
- \\
-\end{array}$ & $\begin{array}{l}0.375 \\
0.476 \\
0.555 \\
0.452 \\
- \\
- \\
- \\
- \\
0.425 \\
0.494 \\
0.526 \\
0.468 \\
0.365 \\
0.590 \\
0.488\end{array}$ & $\begin{array}{c}0.592 \\
0.499 \\
\overline{0} \\
0.455 \\
- \\
- \\
- \\
- \\
0.461 \\
0.540 \\
0.477 \\
0.485 \\
0.389 \\
0.473 \\
0.491\end{array}$ & $\begin{array}{l}0.473 \\
0.488 \\
0.492 \\
0.458 \\
0.385 \\
0.477 \\
0.504 \\
0.442 \\
0.455 \\
0.493 \\
0.506 \\
0.480 \\
0.377 \\
0.531 \\
0.489\end{array}$ & $\mid \begin{array}{cc}15.22 \\
15.25 \\
15 & 84 \\
16 & 27 \\
14 & 68 \\
15.45 \\
15 & 60 \\
16 & 09 \\
15 & 39 \\
15.66 \\
16.55 \\
1684 \\
- \\
-\end{array}$ & $\begin{array}{c}13.41 \\
14.45 \\
14.42 \\
15.76 \\
12.26 \\
15.16 \\
14.87 \\
16.56 \\
13.80 \\
14.87 \\
14.82 \\
16.14 \\
- \\
-\end{array}$ & \begin{tabular}{|}
12.58 \\
15.76 \\
15.93 \\
15.66 \\
- \\
- \\
- \\
- \\
12.95 \\
16.11 \\
16.39 \\
16.19 \\
13.50 \\
16.28 \\
17.39
\end{tabular} \\
\hline Tanne & 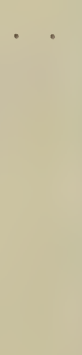 & $18(5)$ & $\begin{array}{r}18.78 \\
14.51 \\
9.79\end{array}$ & $\begin{array}{l}2 \\
3 \\
4 \\
2 \\
3 \\
4 \\
2 \\
3 \\
4\end{array}$ & $\begin{array}{c}0.393 \\
0.482 \\
0.436 \\
0.422 \\
0.496 \\
0.468 \\
- \\
- \\
-\end{array}$ & $\begin{array}{l}0.541 \\
0.429 \\
0.419 \\
0.515 \\
0.521 \\
0.470 \\
0.434 \\
0.456 \\
0.471\end{array}$ & $\begin{array}{c}-\overline{4} \\
0.415 \\
\overline{0} \\
0.444 \\
0.455 \\
-\overline{486} \\
0 .\end{array}$ & $\begin{array}{l}0.397 \\
0.427 \\
0.430 \\
0.467 \\
0.511 \\
0.465 \\
0.471 \\
0.431 \\
0.508\end{array}$ & $\begin{array}{l}0.444 \\
0.437 \\
0.425 \\
0.468 \\
0.493 \\
0.464 \\
0.452 \\
0.458 \\
0.489\end{array}$ & $\begin{array}{c}13.91 \\
14.76 \\
16.19 \\
14.49 \\
14.77 \\
16.37 \\
- \\
-\end{array}$ & $\begin{array}{l}14.93 \\
14.91 \\
16.06 \\
15.24 \\
15.22 \\
16.23 \\
15.85 \\
16.27 \\
17.23\end{array}$ & $\begin{array}{c}- \\
14.44 \\
15.53 \\
- \\
14.73 \\
15.70 \\
- \\
14.45 \\
-\end{array}$ \\
\hline Fichte & & $31(1)$ & $\begin{array}{l}61.60 \\
15.90 \\
13.50 \\
11.68 \\
9.07\end{array}$ & $\begin{array}{l}2 \\
3 \\
4 \\
2 \\
3 \\
4 \\
2 \\
3 \\
4 \\
3 \\
4 \\
2 \\
3 \\
4\end{array}$ & \begin{tabular}{l|}
0.873 \\
0.903 \\
0.979 \\
0.408 \\
0.513 \\
0.463 \\
0.443 \\
0.485 \\
0.463 \\
0.452 \\
0.419 \\
- \\
-
\end{tabular} & $\begin{array}{c}\overline{-} \overline{986} \\
1.013 \\
\overline{4} \\
0.420 \\
0.507 \\
- \\
0.526 \\
0.556 \\
0.552 \\
0.421 \\
- \\
-\end{array}$ & $\begin{array}{l}0.566 \\
0.663 \\
0.701 \\
0.609 \\
0.451 \\
0.517 \\
0.560 \\
0.494 \\
0.473 \\
- \\
-\overline{.} \\
0.488 \\
0.539 \\
0.580\end{array}$ & $\begin{array}{c}0.828 \\
0.959 \\
1.025 \\
0.452 \\
0.427 \\
0.435 \\
0.450 \\
0.472 \\
0.501 \\
- \\
-\overline{4} \\
0.523 \\
0.527\end{array}$ & $\begin{array}{l}0.756 \\
0.878 \\
0.929 \\
0.490 \\
0.453 \\
0.480 \\
0.484 \\
0.494 \\
0.498 \\
0.502 \\
0.420 \\
0.475 \\
0.533 \\
0.553\end{array}$ & $\begin{array}{r}7.35 \\
7.44 \\
7.57 \\
10.99 \\
11.37 \\
12.07 \\
11.68 \\
11.92 \\
12.80 \\
11.64 \\
12.55 \\
- \\
-\end{array}$ & $\begin{array}{c}- \\
7.83 \\
8.26 \\
- \\
10.74 \\
11.55 \\
- \\
11.66 \\
12.21 \\
11.08 \\
11.64 \\
- \\
-\end{array}$ & $\begin{array}{r}8.02 \\
7.24 \\
7.73 \\
8.84 \\
9.25 \\
9.85 \\
9.72 \\
9.99 \\
10.62 \\
- \\
- \\
9.16 \\
9.76 \\
12.06\end{array}$ \\
\hline Fichte & & $31(3)$ & $\begin{array}{r}14.63 \\
14.07 \\
9.96 \\
9.77\end{array}$ & $\begin{array}{l}2 \\
3 \\
2 \\
3 \\
2 \\
3 \\
2 \\
3\end{array}$ & $\begin{array}{c}0.394 \\
0.422 \\
0.417 \\
0.435 \\
- \\
\overline{-} \\
0.423 \\
0.439\end{array}$ & $\begin{array}{c}0.427 \\
0.471 \\
0.438 \\
0.438 \\
- \\
- \\
0.469 \\
0.431\end{array}$ & $\begin{array}{c}- \\
-\overline{429} \\
0.529 \\
0.439 \\
0.489 \\
0.435 \\
0.421\end{array}$ & $\begin{array}{c}\overline{-} \\
\overline{-} \\
0.633 \\
0.421 \\
0.494 \\
0.506 \\
0.430 \\
0.466\end{array}$ & \begin{tabular}{|l|}
0.410 \\
0.446 \\
0.479 \\
0.469 \\
0.466 \\
0.497 \\
0.439 \\
0.439
\end{tabular} & $\left|\begin{array}{c}12.26 \\
12.77 \\
11.76 \\
11.69 \\
- \\
- \\
12.04 \\
12.59\end{array}\right|$ & $\begin{array}{c}- \\
11.98 \\
12.54 \\
12.14 \\
- \\
- \\
13.12 \\
13.24\end{array}$ & $\begin{array}{c}\overline{-} \\
10.96 \\
11.03 \\
11.67 \\
11.69 \\
12.05 \\
11.95\end{array}$ \\
\hline
\end{tabular}




\begin{tabular}{|c|c|c|c|c|c|c|c|c|c|c|c|}
\hline N. & 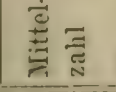 & W. & S. & 0. & $\mathrm{~N}$. & 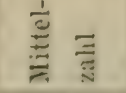 & W. & S. & O. & N. & $\stackrel{\frac{1}{3}}{\equiv}$ \\
\hline 12.76 & 13.49 & 1061.0 & 1052.3 & 667.8 & - & 966.4 & - & - & - & - & - \\
\hline 17.31 & 15.69 & 1209.6 & 1207.7 & 13331.5 & - & 12.49 .6 & - & - & - & - & - \\
\hline 16.14 & $15.5 \mathrm{~s}$ & 1262.1 & 1106.8 & $15 \div 5.9$ & - & 1318.3 & - & - & 一 & - & - \\
\hline 16.77 & 16.11 & 1364.4 & 1308.1 & 12475 & 1440.0 & $1: 340.0$ & - & - & - & - & - \\
\hline - & 13.47 & 914.0 & 666.5 & - & - & 790.2 & 2.98 & - & - & - & 2.98 \\
\hline - & 15.30 & 1273.1 & 1241.5 & - & - & 1257.3 & 3.69 & 3.12 & - & - & 3.41 \\
\hline - & 15.23 & 136.8 & 12666 & - & - & 1317.7 & 4.25 & 4.28 & - & 一 & 4.26 \\
\hline- & 16.32 & 1337.7 & 1311.6 & - & - & 1324.6 & 4.76 & 3.90 & - & - & 4. 30 \\
\hline 13.10 & 13.81 & 1079.0 & 1140.0 & 802.2 & 890.6 & 977.9 & 4.51 & 3.26 & 42.4 & 3.46 & 3.87 \\
\hline 17.61 & 16.06 & 1325.0 & 1141.0 & 14434 & 1885.3 & 1448.7 & 5.33 & 625 & 445 & 5.55 & 5. 39 \\
\hline 16.59 & 16.09 & 1465.0 & 1353.0 & 1590.1 & 1477.1 & 1471.3 & 8.26 & 5.98 & 7.99 & $6: 30$ & 7. 13 \\
\hline 17.17 & 16.58 & 1512.0 & 1447.0 & 1381.2 & 1608.4 & 1487.1 & 782 & 6.95 & 5.56 & 7.01 & 6.83 \\
\hline 15.80 & 14.65 & - & - & 748.5 & 1089.5 & 919.0 & - & - & - & - & - \\
\hline 17.46 & 16.87 & - & - & 1759.3 & 1610.9 & 1685.1 & - & - & - & - & - \\
\hline 17.69 & 17.54 & - & - & 1660.8 & 1729.8 & 1695.3 & - & - & - & - & - \\
\hline 13.86 & 14. 23 & 855.7 & 1132.4 & - & 858.2 & 948.8 & - & - & - & - & - \\
\hline 14.68 & 14.70 & 1181.8 & 1108.3 & 959.8 & 1035.5 & 1071.3 & - & - & - & - & - \\
\hline 16.01 & 15.96 & 1286.2 & 1216.2 & 1126.4 & 1240.3 & 1217.2 & - & - & - & - & - \\
\hline 14.10 & 14.61 & 997.1 & 1345.3 & - & 1044.6 & 1129.0 & 2.67 & 3.64 & - & 4.09 & 3.47 \\
\hline 14.97 & 14.92 & 1217.0 & 1358.2 & $108+.0$ & 1288.2 & 1236.8 & 4.79 & 5.46 & 4.98 & 5.91 & 5.28 \\
\hline 16.39 & 16.17 & 1411.0 & 1393.7 & 1263.0 & 1405.7 & 1368.3 & 7.34 & 5.46 & 5.56 & 5.46 & 5.95 \\
\hline 13.94 & 14.89 & - & 1228.0 & - & 1030.7 & 1129.3 & - & - & 一 & - & - \\
\hline 17.04 & 15.92 & - & 13584 & 1141.8 & 1409.4 & 1303.2 & - & - & - & - & - \\
\hline 16.17 & 16.70 & - & 1572.8 & - & 1496.4 & 1534.6 & - & - & - & - & - \\
\hline 7.91 & 7.76 & 530.4 & - & 410.1 & 582.8 & 507.8 & - & - & - & - & - \\
\hline 7.78 & 7.57 & 561.8 & 680.1 & 390.7 & 653.2 & 571.4 & - & - & - & - & - \\
\hline 7.69 & 7.81 & 631.0 & 778.2 & 471.2 & 681.9 & 640.6 & - & - & - & - & - \\
\hline 10.97 & 10.27 & 554.7 & - & 536.2 & 612.6 & 567.8 & - & - & - & - & - \\
\hline 11.92 & 10.82 & 746.3 & 545.0 & 434.1 & 682.2 & 601.9 & - & - & - & - & - \\
\hline 11.73 & 11.30 & $759: 3$ & 761.4 & 565.2 & 673.3 & 689.8 & - & - & - & - & - \\
\hline 1194 & 11.11 & 679.9 & - & 595.7 & 721.9 & 665.8 & 2.47 & - & 1. 20 & 1.40 & 1.69 \\
\hline 12.62 & 11.55 & 774.9 & 804.8 & 554.4 & 846.3 & 745.1 & 2.00 & 2.24 & 160 & $4+1$ & 2.56 \\
\hline 12.41 & 12.01 & 853.6 & 933.5 & 600.3 & sis. 6 & 814.0 & 2.45 & 3.31 & 3.01 & 2.20 & 2.74 \\
\hline- & 11.36 & 689.0 & 763.0 & - & - & 726.0 & 3.21 & 4.96 & - & - & 4.08 \\
\hline - & 12.09 & 742.2 & 642.4 & - & - & 692.3 & 2.86 & 3.98 & - & 一 & 3.42 \\
\hline 12.58 & 10.87 & - & - & 460.9 & 824.3 & 642.6 & - & - & - & - & - \\
\hline 13.11 & 11.43 & - & - & 577.2 & 1022.4 & 799.8 & -- & 一 & - & - & - \\
\hline 13.00 & 12.53 & - & - & 949.7 & 1002.0 & 975.8 & - & - & - & - & - \\
\hline- & 12.26 & 660.6 & - & - & - & 666.6 & 2.19 & 3.42 & - & - & $\stackrel{2}{2} 81$ \\
\hline - & 12.37 & 774.8 & 761.0 & - & - & 767.9 & 2.64 & 304 & - & - & 2.84 \\
\hline 10.97 & 11.56 & 648.5 & 775.5 & $5 \times 0.1$ & 579.9 & 646.0 & - - & - & - & - & - \\
\hline 10.48 & 11.33 & 669.3 & 762.9 & 590.6 & 5208 & 635.9 & - & - & - & - & - \\
\hline 11.66 & 11.66 & - & -- & 673.5 & 756.2 & 714.8 & - & - & - & - & - \\
\hline 13.05 & 12.37 & - & - & 752.8 & 9704 & 861.6 & - & - & - & - & - \\
\hline 11.80 & 12.25 & 690.5 & 908.5 & 710.6 & 673.3 & 745.7 & 1.66 & 1.65 & 1.61 & 1.61 & 1.63 \\
\hline 11.11 & 12.22 & 783.3 & 850.0 & 677.0 & 647.6 & 7339.5 & 1.93 & 2.35 & 1. 21 & 1.82 & 1.83 \\
\hline
\end{tabular}


Tabelle Die mechanischen Eigenschaften der Jahrringe im Sinne

\begin{tabular}{|c|c|c|c|c|c|c|c|c|c|c|c|c|c|}
\hline \multirow{2}{*}{\multicolumn{5}{|c|}{ Art }} & \multirow{2}{*}{ 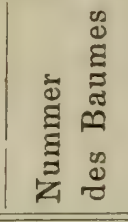 } & \multirow{2}{*}{ 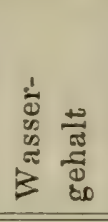 } & \multicolumn{5}{|c|}{ Dichte der Jahrringe } & \multicolumn{2}{|r|}{$\begin{array}{l}\text { Schall- } \\
\text { in den }\end{array}$} \\
\hline & & & & & & & 1 & 2 & 3 & 4 & 5 & 1 & 2 \\
\hline Weissbuche & 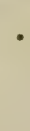 & & . & . $\cdot$ & 7 & $\begin{array}{r}37.22 \\
11.01 \\
8.95\end{array}$ & $\begin{array}{l}0.721 \\
0.571 \\
0.566\end{array}$ & $\begin{array}{l}0.772 \\
0.650 \\
0.594\end{array}$ & $\frac{-}{-}$ & $\frac{-}{-}$ & - & $\begin{array}{r}9.90 \\
12.91 \\
13.30\end{array}$ & \begin{tabular}{|l|}
10.19 \\
12.55 \\
12.96
\end{tabular} \\
\hline Zitterpappel & & . & . & . . & 8 & $\begin{array}{r}38.56 \\
21.83 \\
8.61\end{array}$ & $\begin{array}{l}0.462 \\
0.436 \\
0.421\end{array}$ & $\begin{array}{l}0.467 \\
0.498 \\
0.502\end{array}$ & $\begin{array}{l}0.496 \\
0.486 \\
0.473\end{array}$ & $\begin{array}{l}0.553 \\
0.527 \\
0.521\end{array}$ & I & $\begin{array}{l}11.36 \\
13.33 \\
14.85\end{array}$ & $\begin{array}{l}13.86 \\
15.58 \\
15.16\end{array}$ \\
\hline Erle . . . & & • & . & . . & 12 & $\begin{array}{r}28.55 \\
16.03 \\
9.25\end{array}$ & $\begin{array}{l}0.513 \\
0.490 \\
0.453\end{array}$ & $\begin{array}{l}0.547 \\
0.525 \\
0.501\end{array}$ & $\begin{array}{l}0.522 \\
0.504 \\
0.475\end{array}$ & - & - & $\begin{array}{l}11.17 \\
13.05 \\
15.09\end{array}$ & $\begin{array}{l}14.06 \\
15.12 \\
14.97\end{array}$ \\
\hline Platane. & . & . & . & . & 22 & $\begin{array}{l}31.62 \\
15.08 \\
10.71\end{array}$ & $\begin{array}{l}0.541 \\
0.521 \\
0.505\end{array}$ & $\begin{array}{l}0.605 \\
0.569 \\
0.533\end{array}$ & $\begin{array}{l}0.679 \\
0.611 \\
0.613\end{array}$ & - & - & $\begin{array}{l}11.18 \\
12.53 \\
13.97\end{array}$ & $\begin{array}{l}1314 \\
14.24 \\
14.13\end{array}$ \\
\hline Ahorn . & & · & . & . $\cdot$ & 23 & $\begin{array}{r}29.04 \\
13.79 \\
8.75\end{array}$ & $\begin{array}{l}0.583 \\
0.549 \\
0.518\end{array}$ & $\begin{array}{l}0.630 \\
0.584 \\
0.592\end{array}$ & $\begin{array}{l}0.630 \\
0.602 \\
0.607\end{array}$ & - & - & $\begin{array}{l}1128 \\
12.74 \\
13.90\end{array}$ & $\begin{array}{l}11.41 \\
13.13 \\
13.63\end{array}$ \\
\hline Eiche. . & & . & . & $=$. & 3 & $\begin{array}{r}19.08 \\
17.40 \\
10.96 \\
9.20\end{array}$ & $\begin{array}{c}\overline{0.757} \\
0.749 \\
0.707\end{array}$ & $\begin{array}{l}0.715 \\
0.759 \\
0.774 \\
0.758\end{array}$ & $\begin{array}{l}0.813 \\
0.799 \\
0.789 \\
0.752\end{array}$ & $\begin{array}{l}0.800 \\
0.719 \\
0.743 \\
0.724\end{array}$ & $\begin{array}{l}0.591 \\
0.614 \\
0.627 \\
0.655\end{array}$ & $\begin{array}{c}-\overline{11.44} \\
11.55 \\
15.23\end{array}$ & $\begin{array}{l}12.52 \\
13.21 \\
13.98 \\
13.98\end{array}$ \\
\hline Eiche. . & & . & . & . & $34(3)$ & $\begin{array}{r}17.16 \\
16.63 \\
8.72 \\
8.54\end{array}$ & $\begin{array}{c}0.713 \\
- \\
0.677 \\
0.739\end{array}$ & $\begin{array}{l}0.751 \\
0.765 \\
0.718 \\
9.763\end{array}$ & $\begin{array}{l}0.749 \\
0.711 \\
0.680 \\
0.742\end{array}$ & $\begin{array}{c}0.641 \\
0.629 \\
- \\
0.627\end{array}$ & $\begin{array}{l}- \\
-\end{array}$ & $\begin{array}{c}12.58 \\
- \\
13.76 \\
13.38\end{array}$ & \begin{tabular}{|l|}
12.47 \\
13.01 \\
13.01 \\
13.16
\end{tabular} \\
\hline Eiche. . & & . & . & . . & 45 & $\begin{array}{c}44.83 \\
- \\
19.53 \\
- \\
10.54 \\
- \\
9.16 \\
-\end{array}$ & $\begin{array}{l}0.726 \\
- \\
\overline{-} \\
0.646 \\
- \\
0.692 \\
-\end{array}$ & $\begin{array}{c}0.832 \\
- \\
- \\
\overline{-} \\
0.693 \\
- \\
0.702 \\
-\end{array}$ & $\begin{array}{c}0.852 \\
\overline{-} \\
0.701 \\
- \\
0.699 \\
- \\
-\end{array}$ & $\begin{array}{l}0.685 \\
\overline{-} \\
0.523 \\
\overline{-} \\
0.561 \\
\overline{-} \\
0.713 \\
-\end{array}$ & $\begin{array}{l}0.635 \\
0.605 \\
0.580 \\
0.531 \\
0.554 \\
0.482 \\
0.587 \\
0.523\end{array}$ & $\begin{array}{c}9.84 \\
- \\
\overline{1} \\
\overline{12.14} \\
\overline{12.37} \\
-\end{array}$ & $\begin{array}{c}9.66 \\
- \\
- \\
12.09 \\
- \\
13.12 \\
-\end{array}$ \\
\hline Birke . & & . & - & . & 39 & $\begin{array}{r}37.83 \\
10.67 \\
9.02\end{array}$ & $\begin{array}{l}0.783 \\
0.583 \\
0.580\end{array}$ & $\begin{array}{l}0.751 \\
0.672 \\
0.670\end{array}$ & $\begin{array}{l}0.795 \\
0.721 \\
0.742\end{array}$ & \begin{tabular}{|l|}
0.787 \\
0.736 \\
0.747
\end{tabular} & $\begin{array}{l}0.797 \\
0.742 \\
0.710\end{array}$ & $\begin{array}{l}11.33 \\
15.41 \\
14.91\end{array}$ & $\begin{array}{l}11.52 \\
15.50 \\
15.92\end{array}$ \\
\hline Rothbuche & & . & . & . & $41(1)$ & $\begin{array}{r}19.46 \\
18.22 \\
13.94 \\
9.77\end{array}$ & $\begin{array}{c}0.785 \\
-\overline{7} \\
0.730 \\
0.716\end{array}$ & $\begin{array}{l}0.745 \\
0.707 \\
0.729 \\
0.779\end{array}$ & $\begin{array}{l}0.751 \\
0.737 \\
0.716 \\
0.716\end{array}$ & $\begin{array}{l}0.712 \\
0.707 \\
0.708 \\
0.709\end{array}$ & - & $\begin{array}{r}9.04 \\
- \\
9.73 \\
10.37\end{array}$ & $\begin{array}{l}9.13 \\
9.13 \\
9.65 \\
8.73\end{array}$ \\
\hline Rothbuche & & & . & . & $41(3)$ & $\begin{array}{r}16.50 \\
15.69 \\
11.83 \\
7.63\end{array}$ & $\begin{array}{c}-\overline{0} \\
0.663 \\
0.664 \\
-\end{array}$ & $\begin{array}{l}0.807 \\
0.798 \\
0.775 \\
0.782\end{array}$ & $\begin{array}{l}0.760 \\
0.708 \\
0.744 \\
0.696\end{array}$ & $\begin{array}{l}- \\
-\end{array}$ & $\frac{-}{-}$ & $\begin{array}{l}- \\
9.06 \\
9.23 \\
-\end{array}$ & $\begin{array}{r}9.16 \\
10.48 \\
10.76 \\
10.84\end{array}$ \\
\hline
\end{tabular}


$\mathrm{Nr}$. IX.

der Fasern bei verschiedenen Feuchtigkeitsgraden.

\begin{tabular}{|c|c|c|c|c|c|c|c|c|c|c|c|c|}
\hline \multicolumn{3}{|c|}{$\begin{array}{l}\text { geschwindigkeit } \\
\text { Jahrringen }\end{array}$} & \multicolumn{5}{|c|}{$\begin{array}{c}\text { Elasticitiitscoëfficient in den } \\
\text { Jahrringen }\end{array}$} & \multicolumn{5}{|c|}{$\begin{array}{c}\text { Festigkeit in den Jahrringen } \\
\text { per } \square \text { Millimeter }\end{array}$} \\
\hline 3 & 4 & 5 & 1. & 2 & 3 & 4 & 5 & 1 & 2 & 3 & 4 & 5 \\
\hline - & - & 一 & 795.6 & 902.3 & - & - & - & 2.60 & 2.86 & - & - & - \\
\hline - & - & - & 1070.4 & 1152.1 & - & - & - & 2.82 & 3.31 & - & - & - \\
\hline - & - & - & 1125.6 & 1122.1 & - & - & - & - & - & - & - & - \\
\hline 13.46 & 14.00 & - & 671.2 & 1009.8 & 1010.9 & 1220.0 & - & 1.30 & 4.08 & - & 2.66 & - \\
\hline 15.49 & 16.50 & - & 871.7 & 1359.5 & 1312.0 & 1615.0 & - & 3.18 & 5.54 & 6.60 & 7.60 & - \\
\hline 16.10 & 16.88 & - & 1045.0 & 1298.6 & 1380.0 & 1669.3 & - & - & - & - & - & - \\
\hline 13.80 & - & - & 720.2 & 1216.7 & 1118.8 & - & - & 3.11 & 4.39 & 3.82 & - & - \\
\hline 14 & - & - & 938.9 & 1350.6 & 12 & - & - & 3.16 & 4.53 & 4.96 & - & - \\
\hline 15.38 & - & - & 1162.0 & 1263.6 & 1265.0 & 一 & - & - & - & - & 一 & - \\
\hline 13.54 & - & - & 761.2 & 1174.8 & 1400.4 & - & - & 2.26 & 6.33 & 7.27 & - & - \\
\hline 14.56 & - & - & 920.3 & 1298.9 & 1457.1 & - & - & 3.19 & 7.08 & 5.44 & - & - \\
\hline 13.53 & - & 一 & 1108.7 & 1186.2 & 1263.1 & - & - & - & - & - & - & - \\
\hline 11.74 & - & - & 834.3 & 923.2 & 978.0 & - & - & 1.54 & 1.30 & 1.56 & - & - \\
\hline 13.01 & - & - & 1002.2 & 1136.5 & 1146.3 & - & - & 4.05 & 5.07 & 5.10 & - & - \\
\hline 13.95 & 一 & - & & 1237.8 & 1330.3 & - & - & - & - & - & - & - \\
\hline 14.51 & 13.05 & 12.94 & & 126 & & 1534.1 & 1114.1 & - & 5.06 & 6.90 & 8.90 & 6.47 \\
\hline 13.05 & 12.59 & 12. & & 1488.8 & & 91.7 & & - & - & - & - & - \\
\hline 13.59 & 13.21 & 12 & & 1705.1 & 1654.0 & $1+72.4$ & 1176.0 & 4.31 & 5.26 & 6.72 & 8.87 & 7.69 \\
\hline 13.40 & 14.11 & 12.87 & & 1631.0 & 1535.6 & 1624.3 & 1217.9 & - & - & - & $1-$ & - \\
\hline & $1-1$ & - & - & & & 9 & - & - & -- & - & - & - \\
\hline 12.00 & 12.03 & - & - & 1456.9 & 115 & 1027.8 & - & - & 5.72 & 6.33 & 5.00 & - \\
\hline 11.38 & - & - & 1441.7 & 1368.9 & 123 & - & - & - & - & - & - & - \\
\hline 12.59 & 13.07 & - & 1488.6 & 1497.6 & 1329.7 & 1206.1 & - & 2.72 & 6.94 & 6.68 & 5.93 & - \\
\hline 9.66 & 8.54 & 7 & 790.7 & 877.8 & 889.4 & 575.6 & 449. & - & - & - & - & - \\
\hline- & - & 7 & - & - & - & - & 416. & - & - & - & - & - \\
\hline $\begin{array}{c}10.76 \\
-\end{array}$ & $\begin{array}{c}9.02 \\
-\end{array}$ & 9.62 & - & - & $\begin{array}{c}913.9 \\
-\end{array}$ & 478.4 & 604 & - & - & 5.88 & 5.04 & $\begin{array}{l}3.80 \\
+90\end{array}$ \\
\hline 12.32 & $\overline{10.63}$ & $\begin{array}{r}8.80 \\
10.04\end{array}$ & 1071.0 & 1138.0 & 1193.0 & 728.0 & 63 & $\overline{2.49}$ & $\overline{4.25}$ & 3.83 & $3 . \overline{86}$ & $\begin{array}{l}4.20 \\
3.48\end{array}$ \\
\hline - & - & & - & - & - & - & 52 & - & - & - & - & 2.49 \\
\hline- & 12.41 & 10.84 & 1192.0 & 1364.3 & - & 1237.0 & 783.0 & - & - & - & - & - \\
\hline 一 & - & 10.09 & - & - & - & - & 606.1 & - & - & - & 一 & 一 \\
\hline 12.05 & a & & 30.6 & 1122.4 & 1298.7 & 1098.5 & 97 & 3.89 & - & 4.55 & - & 3.13 \\
\hline 15.41 & 13.38 & & & 1817.0 & 19 & & 1234.4 & 7.03 & 7.18 & 7.01 & 4.14 & 3.15 \\
\hline 14.68 & 13.73 & 11.87 & 1452.0 & 1912.4 & 1802.0 & 1583.7 & 1125.1 & - & 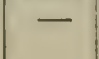 & - & - & - \\
\hline 10.62 & & - & 722.0 & & 960.6 & & - & - & - & - & - & - \\
\hline 10.10 & 11.54 & - & - & 662.5 & 841.6 & 105 & - & - & - & 6.40 & 4.64 & - \\
\hline 11.22 & 12.21 & 一 & 778.1 & 826.5 & 1013.3 & 1188.8 & - & 2.99 & 4.86 & 5.89 & 6.02 & - \\
\hline 11.81 & 11.87 & - & 866.9 & $65+.1$ & 1122.6 & 1125.8 & - & - & - & - & - & - \\
\hline 10.07 & - & - & - & & & - & - & - & 3.95 & 4.14 & - & - \\
\hline 11.59 & - & - & 612.4 & 992.8 & 1070.0 & - & - & - & - & - & - & - \\
\hline 11.90 & - & - & 643.1 & 1018.7 & 1191.9 & - & - & 1.98 & 5.19 & 6.57 & - & - \\
\hline 11.77 & - & - & - & 1038.3 & 1092.4 & - & - & - & - & - & - & - \\
\hline
\end{tabular}




\section{$-90-$
-}

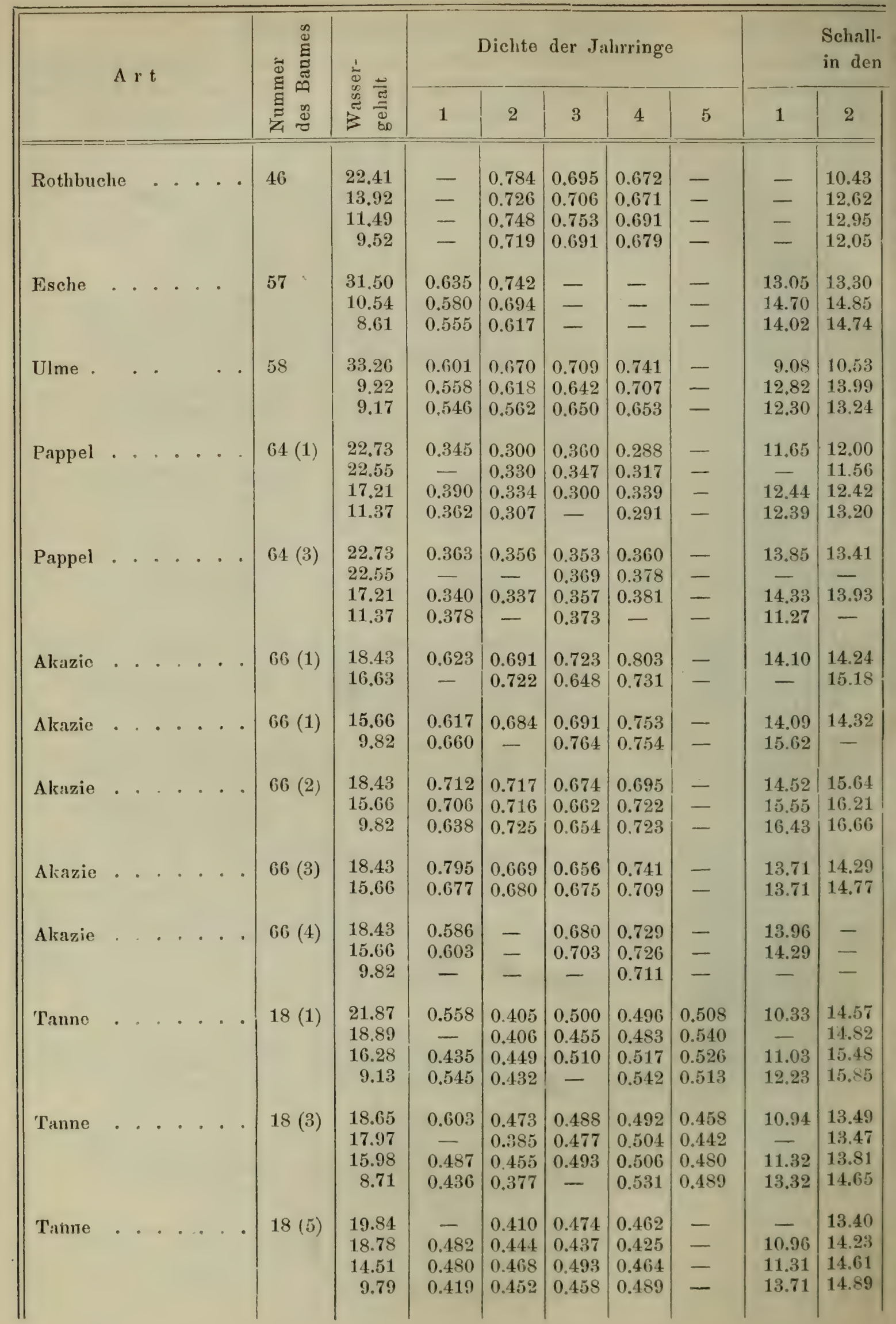




\begin{tabular}{|c|c|c|c|c|c|c|c|c|c|c|c|c|}
\hline \multicolumn{3}{|c|}{$\begin{array}{l}\text { greschwindiglreit } \\
\text { Jahrringen }\end{array}$} & \multicolumn{5}{|c|}{$\begin{array}{l}\text { Elasticitatscoëfficient in den } \\
\text { Jabrringen }\end{array}$} & \multicolumn{5}{|c|}{$\begin{array}{l}\text { Festigkeit in den Jahringes } \\
\text { per } \square \text { Millimeter }\end{array}$} \\
\hline 3 & 4 & 5 & 1 & 2 & 3 & 4 & 5 & 1 & 2 & 3 & 4 & 5 \\
\hline 10.00 & 9.94 & - & - & 959.3 & 732.9 & 748.8 & - & - & - & - & - & - \\
\hline 12.60 & 12.31 & - & - & 1301.7 & 1260.4 & 1147.0 & - & - & 5.61 & 3.60 & 3.57 & - \\
\hline 12.12 & 12.30 & - & - & 1411.3 & 1242.7 & 1175.8 & - & - & 8.20 & 5.41 & 5.11 & - \\
\hline 11.57 & 11.15 & - & - & 1174.1 & 1043.3 & 951.9 & - & - & - & - & - & - \\
\hline- & - & - & 1216.7 & 1476.4 & - & - & - & 4.65 & 6.49 & - & - & - \\
\hline- & - & - & 1409.2 & 1723.0 & - & - & $\cdots$ & 5.68 & 7.71 & - & - & - \\
\hline- & - & - & 1227.9 & 1509.2 & - & - & - & - & $\rightarrow$ & - & - & - \\
\hline 12.51 & 12.07 & - & 558.2 & 836.1 & 1248.7 & 1215.6 & - & 2.07 & 5.74 & 8.89 & 8.59 & - \\
\hline 13.28 & 13.95 & - & 1033.1 & 1361.5 & 1273.9 & 1548.5 & - & - & - & - & - & - \\
\hline 14.46 & 14.62 & - & 929.7 & 1108.4 & 1530.2 & 1569.7 & - & 1.62 & 3.62 & 7.68 & 5.38 & - \\
\hline 11.72 & 11.08 & - & 527.1 & 486.1 & 556.4 & 397.9 & - & - & - & - & - & - \\
\hline 12.19 & 11.05 & - & - & 496.6 & 580.2 & 435.3 & - & - & 2.05 & $2.0 \dot{t}$ & - & - \\
\hline 12.52 & 11.61 & - & 679.0 & 579.9 & 529.0 & 514.4 & - & 3.26 & 2.26 & 2.43 & 1.77 & - \\
\hline- & 11.29 & - & 6249 & 601.3 & - & 417.9 & - & - & - & - & - & - \\
\hline 13.77 & 13.50 & - & 778.0 & 718.4 & 752.5 & 738.3 & - & - & - & - & - & - \\
\hline 12.62 & 13.41 & - & - & - & 661,6 & 765.3 & - & - & - & - & - & - \\
\hline $1+.2: 3$ & 13.72 & - & 785.3 & 735.7 & 813.4 & 807.2 & - & 2.02 & 1.01 & 2.34 & 2.26 & - \\
\hline $1 \tilde{0} .47$ & - & - & 539.8 & - & 1005.6 & - & - & - & - & - & - & - \\
\hline 1.4 .40 & 13.72 & - & 1394.0 & 1577.0 & 1684.3 & 1703.0 & - & - & - & - & - & - \\
\hline 1.4 .41 & 13.68 & - & - & 1871.6 & 1517.8 & 1539.8 & - & - & 8.67 & 8.92 & $9.1 \check{5}$ & - \\
\hline 14.44 & 13.95 & - & 1378.4 & 1578.0 & 1644.1 & 1661.1 & - & 5.09 & 11.61 & 11.03 & 9.62 & - \\
\hline 15.18 & 13.23 & - & 1812,0 & - & 1980.0 & 1486.0 & - & - & - & - & - & - \\
\hline 14.67 & 13.89 & - & 1689.0 & 1973.0 & 1634.0 & 1583.3 & - & - & - & - & - & - \\
\hline 14.82 & 14.04 & - & 1920.0 & 2117.0 & 1637.0 & 1601,3 & - & 4.43 & 10.19 & 10.30 & 9.85 & - \\
\hline 15.92 & 15.76 & - & 1755.0 & 2176.0 & 1887.0 & 2078.0 & - & - & - & - & - & - \\
\hline 14.18 & 14.06 & - & 1682.0 & 1538.0 & 1484.0 & 1650.0 & - & - & - & - & - & - \\
\hline 14.07 & 14.07 & - & 1433.0 & 1669.0 & 1503.0 & 1578.0 & - & 2.47 & 4.14 & 5.76 & 6.91 & - \\
\hline 14.77 & 14.47 & - & 1285.0 & - & 1669.0 & 1717.0 & - & - & - & - & - & - \\
\hline 14.77 & 14.65 & - & 1386.0 & - & 1726.0 & 1752.5 & - & 5.09 & - & 10.19 & 11.88 & - \\
\hline- & 16.13 & - & - & - & - & 2220.0 & - & - & - & - & - & - \\
\hline 15.54 & 14.28 & 15.76 & 670.1 & 967.8 & 1368.2 & 1167.1 & 1422.1 & - & - & - & - & - \\
\hline 15.79 & 14.99 & 15.47 & - & 1004.9 & 1280.2 & 1233.6 & 1455.7 & - & 4.21 & 4.90 & 5.32 & 5.61 \\
\hline 16.29 & 15.03 & 16.46 & 595.6 & 1211.0 & 1541.4 & 1311.1 & 1604.7 & 1.59 & 4.43 & 4.66 & 4.87 & 8.41 \\
\hline 16.40 & 14.81 & 17.01 & 916.9 & 1220.7 & 1864.0 & 1359.6 & 1670.1 & - & - & - & - & - \\
\hline 15.69 & 15.58 & 16.11 & $\$ 12.1$ & 966.4 & 1249.6 & 1318.3 & 1340.0 & - & - & - & - & - \\
\hline 15.30 & 15.23 & 16.32 & - & 790.2 & 1257.3 & 1317.7 & 1324.6 & - & -2.98 & 3.41 & 4.26 & 4.33 \\
\hline 16.06 & 16.09 & 16.58 & 702.9 & 977.9 & 1448.7 & 1471.3 & 1487.1 & 3.89 & 3.87 & $\check{5} .39$ & 7.13 & 6.83 \\
\hline- & 16.87 & 17.54 & 870.3 & 919.0 & - & 1685.1 & 1695.3 & - & - & - & - & - \\
\hline 15.04 & 16.32 & - & - & 828.5 & 1207.2 & 1384.6 & - & - & 2.12 & 2.71 & 4.13 & - \\
\hline 14.70 & 15.96 & - & 651.5 & 948.8 & 1071.3 & 1217.2 & - & $\longrightarrow$ & - & - & - & - \\
\hline 14.92 & 16.17 & - & 691.3 & 1129.0 & 1236.8 & 1368.3 & - & 2.38 & 3.47 & 5.28 & 5.95 & - \\
\hline 15.92 & 16.70 & - & 894.3 & 1129.3 & 1303.2 & 1534.6 & - & - & - & - & 7 & - \\
\hline
\end{tabular}




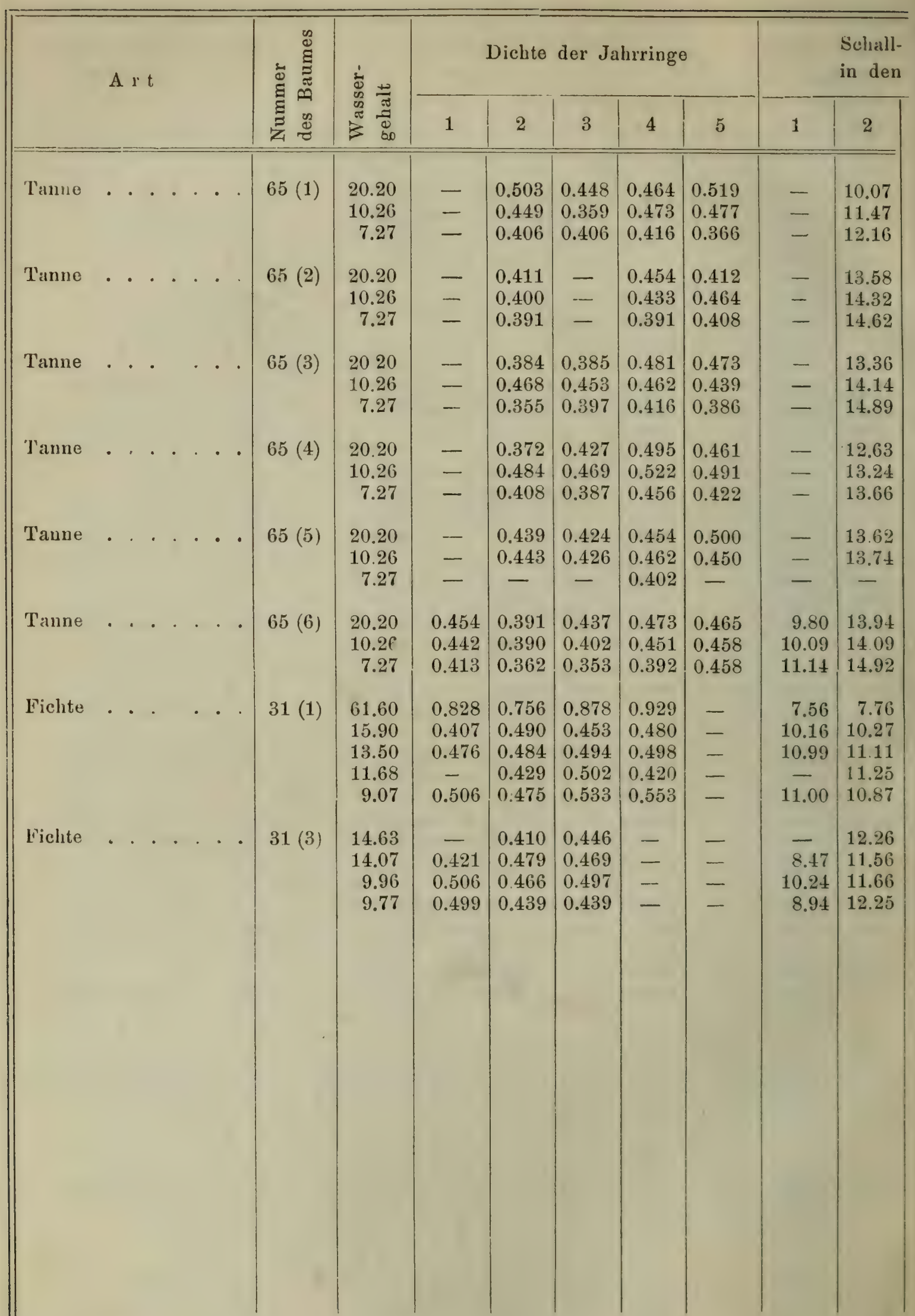

Note. Bei der Eiche Nr. 45 (Seite 88 und 89) sind die auf den Jahrring 6 


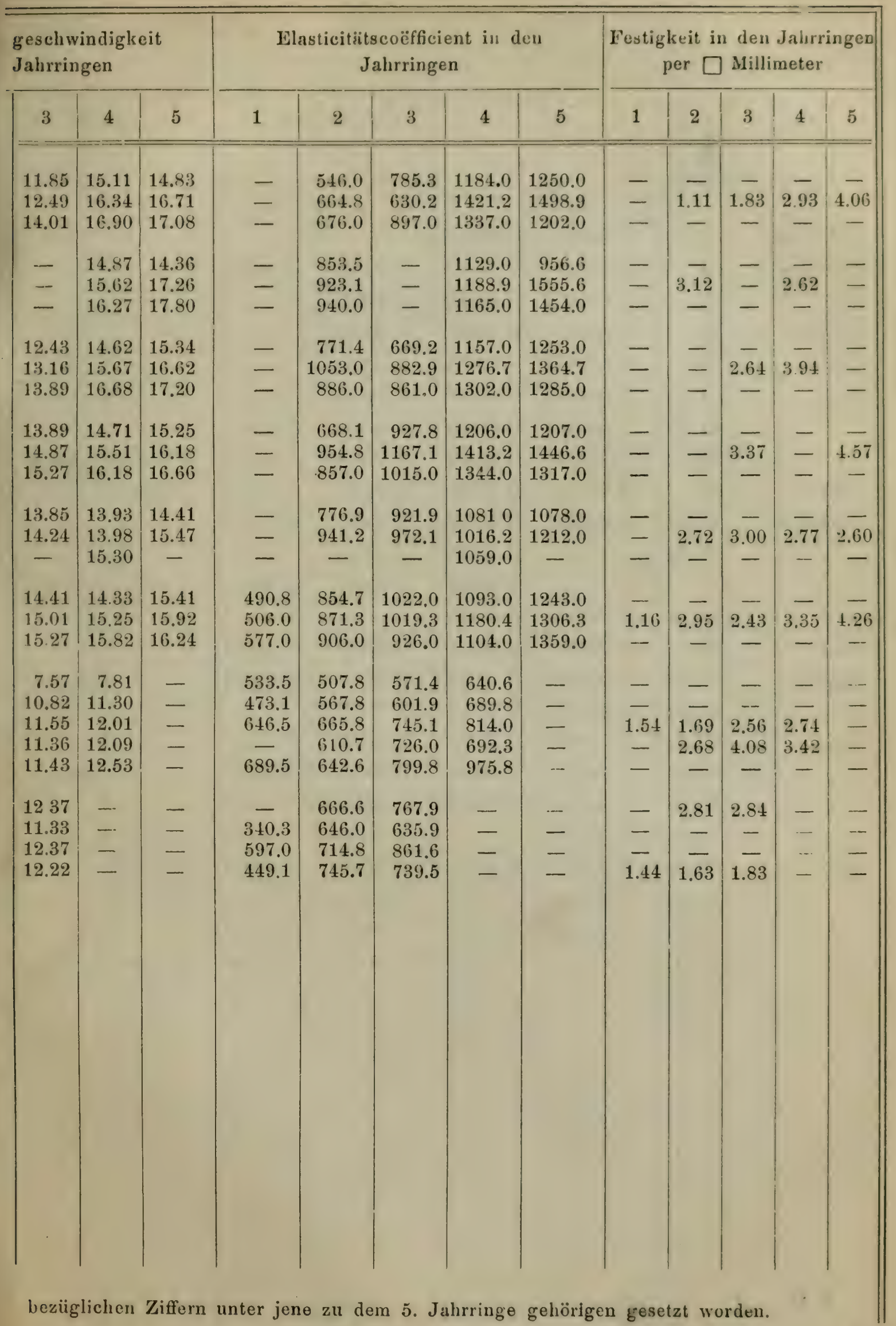


Tabelle

Variationscoëfficient der Dichte und Schallgeschwindigkeit

\begin{tabular}{|c|c|c|c|c|c|c|c|c|}
\hline \multirow{2}{*}{\multicolumn{2}{|c|}{ Art }} & \multirow{2}{*}{ 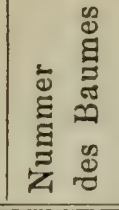 } & \multicolumn{6}{|c|}{$\begin{array}{l}\text { Variationscoëficient der Dichte in den } \\
\text { Jahrringen }\end{array}$} \\
\hline & & & 1 & 2 & 3 & 4 & 5 & 6 \\
\hline \multicolumn{2}{|c|}{ Weissbuche } & 7 & 0.00777 & 0.00709 & - & - & - & - \\
\hline \multicolumn{2}{|c|}{ 'Zitterpappel } & 8 & 0.00316 & - & 0.00137 & 0.00237 & - & - \\
\hline \multicolumn{2}{|c|}{ Erle . . } & 12 & 0.00482 & 0.00378 & 0.00370 & - & - & - \\
\hline \multicolumn{2}{|c|}{ Sycomore. } & 22 & 0.00270 & 0.00464 & 0.00535 & - & - & - \\
\hline \multicolumn{2}{|c|}{ Ahorn . } & 23 & 0.00465 & 0.00388 & 0.00235 & - & - & - \\
\hline \multicolumn{2}{|c|}{ Eiche. . . } & $34(1)$ & 0.00484 & 0.00015 & 0.00455 & - & - & - \\
\hline \multicolumn{2}{|l|}{$"$} & $34(3)$ & 0.00598 & 0.00521 & 0.00599 & 0.00253 & - & - \\
\hline \multicolumn{2}{|l|}{$"$. } & 45 & 0.00226 & 0.00462 & 0.00612 & 0.00450 & 0.00309 & 0.00485 \\
\hline \multicolumn{2}{|c|}{ Birke . . } & 39 & 0.00920 & 0.00380 & 0.00287 & 0.00207 & 0.00316 & - \\
\hline \multicolumn{2}{|c|}{ Rothbuche } & $41(1)$ & 0.01088 & 0.00389 & 0.00662 & 0.00072 & - & - \\
\hline \multicolumn{2}{|l|}{$n$} & $41(3)$ & - & 0.00498 & 0.00210 & - & - & - \\
\hline \multicolumn{2}{|l|}{$n$} & 46 & - & 0.00645 & 0.00045 & 0.00017 & - & - \\
\hline \multicolumn{2}{|l|}{ Esche } & 57 & 0.00481 & 0.00522 & - & - & - & - \\
\hline \multicolumn{2}{|l|}{ Ulme. } & 58 & 0.00339 & 0.00496 & $\cdot 0.00369$ & 0.00342 & - & - \\
\hline \multicolumn{2}{|l|}{ Pappel } & $64(1)$ & +0.00434 & +0.00205 & 0.03019 & +0.00092 & 一 & - \\
\hline \multicolumn{2}{|l|}{$n$} & $64(3)$ & 0.00392 & 0.00967 & +0.00499 & - & - & - \\
\hline \multicolumn{2}{|c|}{ Aksizio. } & $66(1)$ & 0.00348 & 0.00366 & 0.01598 & 0.01478 & - & - \\
\hline$"$ & . & $66(2)$ & 0.00755 & 0.00050 & 0.00494 & +0.00935 & - & - \\
\hline \multicolumn{2}{|l|}{$"$} & $66(3)$ & 0.05358 & +0.00593 & +0.00104 & 0.01559 & - & - \\
\hline \multicolumn{2}{|l|}{$"$} & $66(4)$ & +0.01047 & - & +0.01221 & 0.00218 & - & - \\
\hline \multicolumn{2}{|l|}{ Tanno } & $18(1)$ & 0.02063 & +0.00883 & 0.03020 & 0.00879 & +0.00942 & - \\
\hline \multicolumn{2}{|l|}{$"$} & $18(3)$ & 0.04995 & 0.01733 & 0.03315 & +0.00931 & +0.01240 & - \\
\hline \multicolumn{2}{|l|}{$"$} & $18(5)$ & 0.00775 & +0.00733 & 0.07364 & 0.07556 & - & - \\
\hline$"$ & . . & $65(1)$ & - & 0.01285 & 0.01361 & 0.00800 & 0.01547 & - \\
\hline$"$ & . . & $65(2)$ & - & 0.00320 & - & 0.00769 & 0.00075 & - \\
\hline$"$ & . . & $65(3)$ & - & 0.00584 & +0.01009 & 0.00721 & 0.01072 & - \\
\hline$"$ & . . & $65(4)$ & - & +0.01888 & 0.00724 & 0.00609 & 0.00654 & - \\
\hline$"$ & . . & $65(5)$ & - & +0.00092 & +0.00047 & 0.00886 & 0.01006 & - \\
\hline$n$ & . . & $65(6)$ & 0.00482 & 0.00300 & 0.01146 & 0.00896 & 0.00133 & - \\
\hline Fichte & . . & $31(1)$ & 0.00912 & 0.00773 & 0.00893 & 0.00972 & - & - \\
\hline$n$ & . . & $31(3)$ & - & 0.01301 & 0.01488 & - & - & - \\
\hline
\end{tabular}


$\mathrm{Nr} \cdot \mathrm{X}$.

in den Jahrringen bei Verlust von $1 \%$ Feuchtigkoit.

\begin{tabular}{|c|c|c|c|c|c|c|c|}
\hline \multirow{2}{*}{ Mittelzahl } & \multicolumn{6}{|c|}{$\begin{array}{l}\text { Variationscoëficient der Schallgeschwindigkeit } \\
\text { in den Jahringen }\end{array}$} & \multirow{2}{*}{ Mittelzalıl } \\
\hline & 1 & 2 & 3 & 4 & 5 & 6 & \\
\hline 0.00748 & 0.01187 & 0.00922 & - & - & - & - & 0.00951 \\
\hline 0.00230 & 0.01031 & 0.00527 & 0.00778 & 0.00877 & - & - & 0.00803 \\
\hline 0.00410 & 0.01581 & 0.00468 & 0.00641 & - & - & - & 0.00897 \\
\hline 0.00123 & 0.00961 & 0.00433 & 0.00226 & - & - & - & 0.00540 \\
\hline 0.00363 & 0.00996 & 0.00973 & 0.00818 & - & - & - & 0.00929 \\
\hline \multirow{3}{*}{0.00420} & 0.02094 & 0.00808 & 0.00484 & 0.01118 & 0.00815 & - & \multirow{3}{*}{0.00805} \\
\hline & 0.00924 & 0.00577 & 0.00008 & 0.00746 & - & - & \\
\hline & 0.00701 & 0.00868 & 0.00626 & 0.00735 & 0.00882 & 0.00691 & \\
\hline \multirow[t]{2}{*}{0.00422} & 0.01212 & 0.01299 & 0.00892 & 0.00773 & 0.00540 & - & \multirow{4}{*}{$\begin{array}{l}0.00943 \\
0.01068\end{array}$} \\
\hline & 0.01450 & 0.00290 & 0.01089 & 0.00686 & - & - & \\
\hline \multirow[t]{2}{*}{$0.00486^{\circ}$} & 0.00486 & 0.00559 & 0.00443 & - & - & - & \\
\hline & - & 0.01963 & 0.02074 & 0.01642 & - & - & \\
\hline 0.00501 & 0.00464 & 0.00514 & - & - & - & - & 0.00489 \\
\hline 0.00386 & 0.01593 & 0.01217 & 0.00451 & 0.00762 & - & - & 0.01006 \\
\hline \multirow{2}{*}{0.00450} & 0.00893 & 0.00757 & 0.01236 & 0.00516 & - & - & \multirow{2}{*}{0.00592} \\
\hline & -0.00506 & 0.00702 & 0.00846 & 0.00295 & - & - & \\
\hline \multirow{4}{*}{0.00555} & 0.00613 & 0.00203 & 0.00364 & 0.00095 & - & - & \multirow{4}{*}{0.00576} \\
\hline & 0.02044 & 0.01036 & 0.00679 & 0.00977 & - & - & \\
\hline & - & 0.01213 & -0.00280 & 0.00026 & - & - & \\
\hline & 0.00853 & - & -- & 0.00811 & - & - & \\
\hline \multirow{9}{*}{0.01034} & 0.01328 & 0.00461 & 0.00612 & 0.00966 & 0.00266 & - & \multirow{9}{*}{0.00797} \\
\hline & 0.01745 & 0.00876 & 0.00883 & 0.01029 & 0.00992 & - & \\
\hline & 0.01769 & 0.00570 & 0.00685 & 0.00412 & - & - & \\
\hline & - & 0.01502 & 0.00976 & 0.00867 & 0.01224 & 一 & \\
\hline & - & 0.00570 & - & 0.00617 & 0.01942 & $\cdots$ & \\
\hline & - & 0.00736 & 0.00750 & 0.00906 & 0.00888 & - & \\
\hline & --- & 0.00558 & 0.00739 & 0.00660 & 0.00664 & - & \\
\hline & - & 0.00089 & 0.00283 & 0.00398 & 0.00740 & - & \\
\hline & 0.00677 & 0.00326 & 0.00480 & 0.00725 & 0.00374 & - & \\
\hline \multirow{2}{*}{0.01056} & 0.00854 & 0.00817 & 0.01001 & 0.01086 & - & - & \multirow{2}{*}{0.01369} \\
\hline & 0.03187 & 0.00799 & 0.02030 & - & - & - & \\
\hline
\end{tabular}




\section{Tabelle Nr. XI.}

Lineare Transversalschwindung der Hölzer in Folge ihrer Austrocknung.

\begin{tabular}{|c|c|c|c|c|c|c|c|c|c|c|c|}
\hline & $r t$ & & & & & 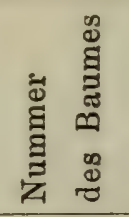 & 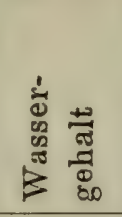 & 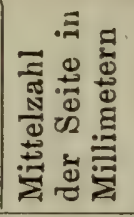 & $\begin{array}{c}\text { Lineare } \\
\text { Schwindung } \\
\text { für } 1 \% \\
\text { Wasserverlust }\end{array}$ & \multicolumn{2}{|c|}{ Mittelzahl } \\
\hline Weissbuche & . & & . & & . . & 7 & $\begin{array}{r}37.22 \\
11.01 \\
8.95\end{array}$ & $\begin{array}{l}6.84 \\
6.51 \\
6.62\end{array}$ & $\left.\begin{array}{l}0.00184 \\
0.00114\end{array}\right\}$ & 0.00149 & \\
\hline Zitterpappel & . & . & - & . & . . & 8 & $\begin{array}{r}38.56 \\
21.83 \\
8.61\end{array}$ & $\begin{array}{l}7.22 \\
6.74 \\
6.41\end{array}$ & $\left.\begin{array}{l}0.00397 \\
0.00374\end{array}\right\} \ldots$ & 0.00385 & \\
\hline Erle . . & • & . & . & . . & . . & 12 & $\begin{array}{r}28.55 \\
16.03 \\
9.25\end{array}$ & $\begin{array}{l}6.95 \\
6.65 \\
6.66\end{array}$ & $\left.\begin{array}{l}0.00345 \\
0.00216\end{array}\right\} \cdots$ & 0.00280 & \\
\hline Sycomore . & . & . & . & . & . . & 22 & $\begin{array}{l}31.62 \\
15.08 \\
10.71\end{array}$ & $\begin{array}{l}6.97 \\
6.59 \\
6.54\end{array}$ & $\left.\begin{array}{l}0.00329 \\
0.00295\end{array}\right\} \ldots$ & 0.00312 & \\
\hline Ahorn . & . & . & - & . & . . & 23 & $\begin{array}{r}29.04 \\
13.79 \\
8.75\end{array}$ & $\begin{array}{l}7.29 \\
6.92 \\
6.81\end{array}$ & $\left.\begin{array}{l}0.00333 \\
0.00324\end{array}\right\} \cdots$ & 0.00328 & \\
\hline Eiche... & . & . & . & & . . & 34 (1) & $\begin{array}{r}19.08 \\
17.40 \\
10.96 \\
9.20\end{array}$ & $\begin{array}{l}7.07 \\
7.02 \\
6.74 \\
6.72\end{array}$ & $\left.\begin{array}{l}0.00421 \\
0.00575 \\
0.00501\end{array}\right\}$ & 0.00499 & \\
\hline Eiche... & . & . & . & . & . . & $34(3)$ & $\begin{array}{r}17.16 \\
8.54\end{array}$ & $\begin{array}{l}7.00 \\
668\end{array}$ & $0.00530 \ldots$ & 0.00530 & 0.00461 \\
\hline Eiche.. & . & . & . & . & . . & 45 & $\begin{array}{l}44.83 \\
19.53 \\
10.54\end{array}$ & $\begin{array}{r}11.10 \\
10.00 \\
9.90\end{array}$ & $\left.\begin{array}{l}0.00392 \\
0.00315\end{array}\right\} \cdots$ & 0.00353 & \\
\hline Birke . . & . . & . & . & . & . . & 39 & $\begin{array}{r}37.83 \\
10.67 \\
9.02\end{array}$ & $\begin{array}{l}7.25 \\
6.58 \\
6.51\end{array}$ & $\left.\begin{array}{l}0.00340 \\
0.00354\end{array}\right\} \cdots$ & 0.00347 & \\
\hline Rothbuche & . & . & : & . & . . & $41(1)$ & $\begin{array}{r}19.46 \\
13.94 \\
9.77\end{array}$ & $\begin{array}{l}6.96 \\
6.84 \\
6.64\end{array}$ & $\left.\begin{array}{l}0.00312 \\
0.00474\end{array}\right\} \cdots$ & 0.00393 & \\
\hline Rothbuche & . & . & . & - & . . & $41(3)$ & $\begin{array}{r}16.50 \\
11.83 \\
7.63\end{array}$ & $\begin{array}{l}6.82 \\
6.75 \\
6.71\end{array}$ & $\left.\begin{array}{l}0.00220 \\
0.00182\end{array}\right\} \cdots$ & 0.00201 & 0.00412 \\
\hline Rothbuche & . & . & . & . & . . & 46 & $\begin{array}{r}22.41 \\
13.92 \\
11.49 \\
9.52\end{array}$ & $\begin{array}{l}7.23 \\
6.82 \\
6.67 \\
6.72\end{array}$ & $\left.\begin{array}{l}0.00668 \\
0.00709 \\
0.00547\end{array}\right\}$ & $0.006+1$ & \\
\hline
\end{tabular}




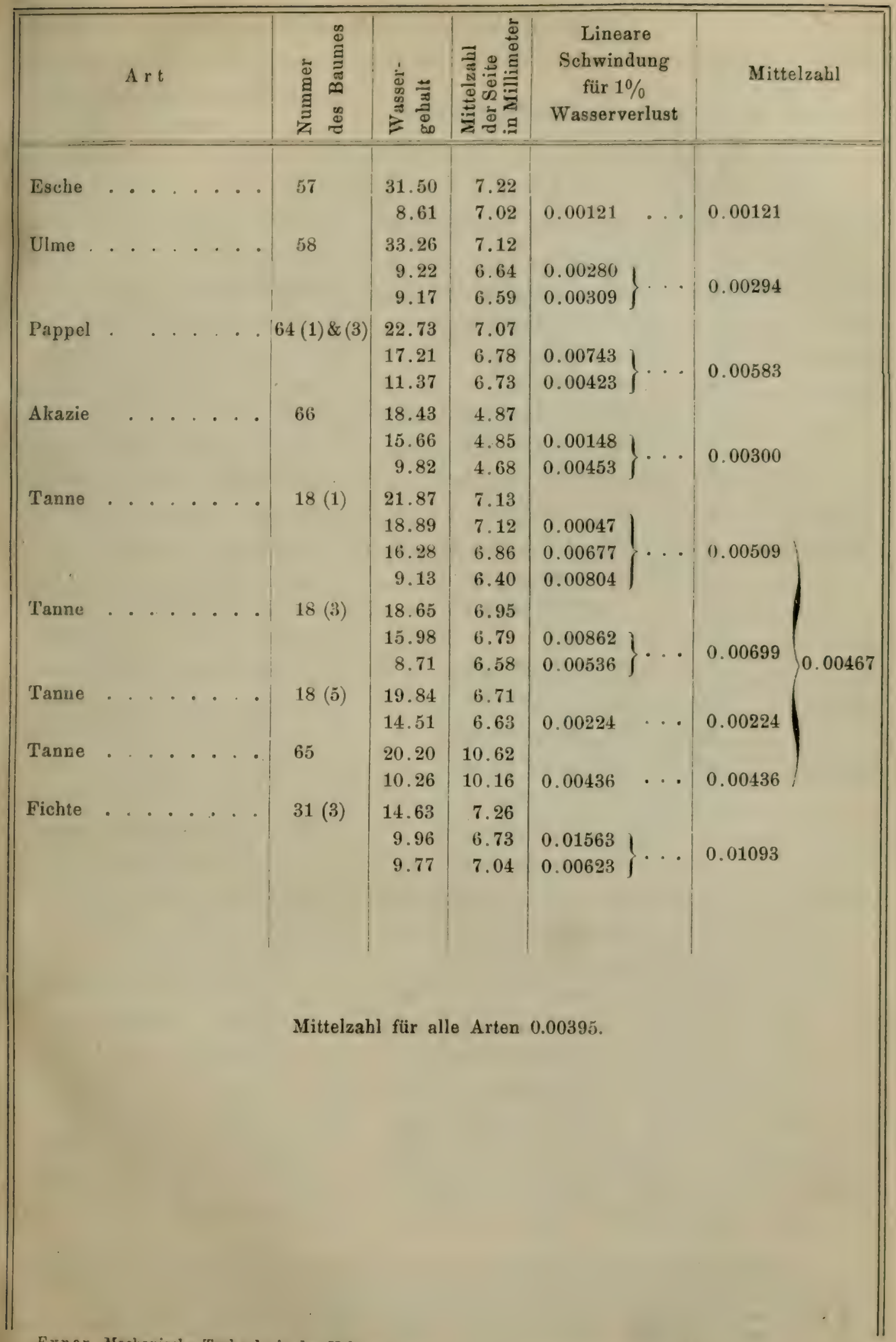




\section{Dichte, wirklicher Elasticitätscoëfficient und Festig-}

\begin{tabular}{|c|c|c|c|c|c|c|c|c|c|c|c|c|c|c|c|c|}
\hline \multirow{2}{*}{\multicolumn{4}{|c|}{ A r t }} & \multirow{2}{*}{ 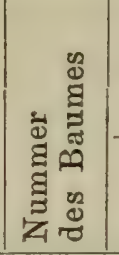 } & \multicolumn{6}{|c|}{ Dichte der Jahrringe } & \multicolumn{6}{|c|}{$\begin{array}{c}\text { Schallgeschwindigkeit in den } \\
\text { Jahrringen }\end{array}$} \\
\hline & & & & & 1 & 2 & 3 & 4 & 5 & 6 & 1 & 2 & 3 & 4 & 5 & 6 \\
\hline Weissbr & ach & & & 7 & 0.622 & 0.692 & - & - & - & - & 11.87 & 11.74 & - & - & - & - \\
\hline Zitterpa & ppe & & & 8 & 0.434 & 0.499 & 0.484 & 0.526 & - & - & 13.54 & 15.52 & 15.58 & 16.55 & - & - \\
\hline Erle & . & • & & 12 & 0.497 & 0.532 & 0.510 & - & - & - & 12.45 & 14.78 & 14.61 & - & - & - \\
\hline Sycomo & re & . & & 22 & 0.527 & 0.579 & 0.631 & - & - & - & 12.12 & 13.91 & 14.26 & - & - & - \\
\hline Ahorn & . & • & & 23 & 0.563 & 0.603 & 0.613 & . - & - & - & 12.15 & 12.43 & 12.49 & - & - & - \\
\hline Eiche. & . & & & 45 & 0.668 & 0.731 & 0.704 & 0.526 & 0.581 & 0.532 & 11.50 & 11.42 & 10.74 & 9.01 & 9.59 & 8.78 \\
\hline$n \quad \cdot$ & - & • & & $34(1)$ & 0.760 & 0.708 & 0.816 & 0.806 & 0.587 & - & 11.39 & 12.35 & 14.61 & 13.03 & 12.95 & - \\
\hline$" \quad$. & . & 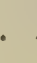 & & $34(3)$ & 0.704 & 0.747 & 0.751 & 0.646 & - & - & 12.31 & 12.24 & 11.76 & 12.02 & - & - \\
\hline Birke. & . & . & & 39 & 0.652 & 0.699 & 0.746 & 0.754 & 0.761 & - & 14.01 & 14.13 & 14.26 & 12.61 & 11.57 & - \\
\hline Rothbuc & he & & & 41 (1) & 0.790 & 0.747 & 0.754 & 0.712 & - & - & 8.97 & 9.08 & 10.56 & 11.46 & - & - \\
\hline$"$ & & & & $41(3)$ & 0.662 & 0.824 & 0.663 & - & - & - & 8.87 & 9.14 & 10.11 & - & - & - \\
\hline$n$ & & • & & 46 & - & 0.768 & 0.698 & 0.672 & - & - & - & 11.05 & 10.74 & 10.61 & - & - \\
\hline Esche & • & & & 57 & 0.605 & 0.716 & - & - & - & - & 1395 & 14.15 & - & - & 一 & - \\
\hline Ulme . & . & 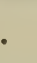 & . & 58 & 0.577 & 0.641 & $0.672 \mid$ & 0.722 & - & - & 11.14 & 12.44 & 12.93 & 13.11 & - & - \\
\hline Pappel & - & . & & $64(1)$ & 0.367 & 0.316 & 0.330 & 0.313 & - & - & 12.04 & 12.21 & 12.12 & 11.34 & - & - \\
\hline$\eta$ & $\cdot$ & • & & $64(3)$ & 0.352 & 0.347 & 0.355 & 0.370 & - & - & 14.09 & 13.67 & 14.01 & 13.61 & - & - \\
\hline Akazie & . & • & & $66(1)$ & 0.626 & 0.697 & 0.741 & 0.831 & - & - & 14.11 & 14.19 & 14.38 & 13.59 & - & - \\
\hline$"$ & . & . & & $66(2)$ & 0.715 & 0.718 & 0.681 & 0.680 & - & - & 13.94 & 15.32 & 14.58 & 13.80 & - & - \\
\hline$"$ & . & - & & $66(3)$ & 0.862 & 0.663 & 0.645 & 0.759 & - & - & 13.71 & 14.02 & 14.24 & 14.05 & - & - \\
\hline$n$ & $\cdot$ & - & & $66(4)$ & 0.576 & 0.621 & 0.667 & 0.731 & - & - & 13.77 & - & 14.77 & 14.37 & - & - \\
\hline T'anne & . & • & & $18(1)$ & 0.517 & 0.406 & 0.471 & 0.488 & 0.528 & - & 11.65 & 14.72 & 15.70 & 14.72 & 15.55 & - \\
\hline$"$ & . & . & & $18(3)$ & 0.662 & 0.482 & 0.485 & 0.485 & 0.447 & - & 10.75 & 13.33 & 15.50 & 15.32 & 15.87 & - \\
\hline$n$ & . & . & & $18(5)$ & 0.483 & 0.405 & 0.480 & 0.468 & - & - & 10.86 & 13.36 & 15.10 & 16.37 & - & - \\
\hline$"$ & $\cdot$ & - & & $65(1)$ & - & 0.502 & 0.446 & 0.466 & 0.518 & - & - & 10.36 & 11.98 & 15.13 & 14.88 & - \\
\hline$"$ & - & - & & $65(2)$ & - & 0.411 & 0.432 & 0.453 & 0.413 & - & - & 13.59 & - & 14.88 & 14.42 & - \\
\hline$"$ & . & . & & $65(3)$ & - & 0.386 & 0.387 & 0.481 & 0.472 & - & - & 13.40 & 12.44 & 14.63 & 15.36 & - \\
\hline$"$ & 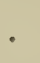 & • & & $65(4)$ & - & 0.374 & 0.428 & 0.495 & 0.462 & - & - & 12.75 & 13.91 & 14.72 & $|15.37|$ & - \\
\hline$"$ & $\cdot$ & - & & $65(5)$ & - & 0.439 & 0.424 & 0.454 & 0.499 & - & - & 13.62 & 13.86 & 13.93 & 14.43 & - \\
\hline$r$ & . & - & & $65(6)$ & 0.454 & 0.391 & 0.436 & 0.472 & 0.465 & - & 9.80 & 13.94 & 14.42 & 14.35 & 15.42 & - \\
\hline Fichte & . & $\cdot$ & & 31 (1) & 0.445 & 0.514 & 0.491 & 0.521 & - & - & 9.92 & 10.05 & 10.53 & 10.99 & - & - \\
\hline$"$ & . & . & & $31(3)$ & 0.313 & 0.534 & $=0.510$ & - & - & - & 7.82 & 10.61 & 10.10 & - & - & - \\
\hline
\end{tabular}

Da die Breite der Jahrringe bei der Eiche 45, der Rothbuche 46, bei den Akazien 66 und don Tannen 65 Columnen werden erhalten, indem man die den einzelnen Jahrringen entsprechenden Zahlen für Dichte. Elasticitat tenen Produkto summirt und durch das Quadrat der Anzahl der in Rechumg gezegenen Jahringe dividirt. In den zahl weniger dem Quadrat von 1 oder 2 zu nehmen. Da bei der Akazio 66 (4) und bei der Tamne 65 (2) eine der 
Nr. XII.

keit im Sinne der Faser bei 20\% Fouchtigkeit.

\begin{tabular}{|c|c|c|c|c|c|c|c|c|c|c|c|c|c|c|}
\hline \multicolumn{6}{|c|}{$\begin{array}{l}\text { Elasticitätscoëfficient in den } \\
\text { Jahrringen }\end{array}$} & \multicolumn{6}{|c|}{ Festigkeit in den Jahrringen } & \multicolumn{3}{|c|}{$\begin{array}{l}\text { Mittelzahl für die } \\
\text { ganze Baummasse }\end{array}$} \\
\hline 1 & 2 & 3 & 4 & 5 & 6 & 1 & 2 & 3 & 4 & 5 & 6 & 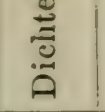 & 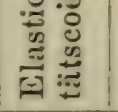 & 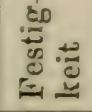 \\
\hline 892.5 & 971.4 & - & - & - & - & 2.74 & 3.16 & - & - & - & - & 0.587 & 966.3 & 2.99 \\
\hline 865.1 & 1306.9 & 1277.6 & 1566.5 & - & - & 3.38 & 5.70 & 7.00 & 8.14 & - & - & 0.501 & 1399.8 & 7.20 \\
\hline 773.4 & 1166.7 & 10929 & - & - & - & 3.14 & 4.49 & 4.60 & - & - & - & 0.514 & 1100.4 & 4.54 \\
\hline 764.9 & 1106.9 & 1267.8 & - & - & - & 2.91 & 6.86 & 5.98 & - & - & - & 0.611 & 1199.0 & 6.16 \\
\hline 875.8 & 981.7 & 1007.7 & - & - & - & 3.02 & 3.53 & 3.66 & - & - & - & 0.607 & 997.1 & 3.58 \\
\hline 882.1 & 951.8 & 810.9 & 426.1 & 533.7 & 409.5 & 3.19 & 5.45 & 5.97 & 5.12 & 3.82 & 4.24 & 0.573 & 557.8 & 4.62 \\
\hline 1006.9 & 1102.8 & 1778.7 & 1397.5 & 1005.3 & - & 4.08 & 5.04 & 6.92 & 8.90 & 6.36 & - & 0.719 & 1287.2 & 7.41 \\
\hline 1070.0 & 1122.5 & 1041.8 & 936.2 & - & - & 2.23 & 5.33 & 6.20 & 4.68 & - & - & 0.697 & 994.6 & 5.33 \\
\hline 1188.3 & 1296.3 & 1408.5 & 1113.2 & 945.9 & - & 5.50 & 6.48 & 5.91 & 3.58 & 3.14 & - & 0.749 & 114.5 .3 & 4.30 \\
\hline 678.7 & 657.6 & 897.7 & 998.4 & - & - & 2.59 & 4.21 & 6.64 & 4.24 & - & - & 0.742 & 857.4 & 5.25 \\
\hline 568.0 & 716.8 & 739.0 & - & - & - & 1.11 & 3.35 & 3.24 & - & - & - & 0.735 & 682.8 & 3.23 \\
\hline - & 849.7 & 729.5 & 685.5 & - & - & - & 3.13 & 1.96 & 2.04 & - & - & 0.700 & 718.4 & 2.23 \\
\hline 1063.8 & 1294.4 & - & - & - & - & 5.22 & 7.16 & - & - & - & - & .694 & 1249.0 & 6.78 \\
\hline 544.8 & 949.7 & 1076.0 & 1188.5 & - & - & 1.82 & 4.57 & 8.22 & 6.82 & - & - & 0.685 & 1087.7 & 6.99 \\
\hline 624.9 & 553.4 & 569.4 & 472.7 & - & - & 3.03 & 2.15 & 2.21 & 1.64 & - & - & 0.324 & 554.2 & 2.13 \\
\hline 751.1 & 697.0 & 749.0 & 736.7 & - & - & 1.88 & 0.94 & 2.17 & 2.10 & - & - & 0.357 & 705.8 & 1.82 \\
\hline 1175.7 & 1323.9 & 1445.4 & $=1447.8$ & - & - & 2.63 & 4.61 & 5.36 & 7.82 & - & - & 0.765 & 1406.8 & 6.12 \\
\hline 1310.6 & 1589.7 & 1365.6 & 1221.6 & - & - & 4.43 & 10.19 & 10.30 & 9.85 & 5 & - & 0.690 & 1341.2 & 9.71 \\
\hline 1528.4 & 1229.3 & 1233.8 & 1413.4 & - & - & 2.47 & 4.14 & 5.76 & 6.91 & - & - & 0.712 & 1329.9 & 5.75 \\
\hline 1030.3 & 1201.4 & 1372.6 & 1423.9 & - & - & 5.09 & 7.64 & 10.19 & 11.88 & - & - & 0.681 & 1341.5 & 10.13 \\
\hline 704.4 & 883.2 & 1165.5 & 1061.5 & 1286.6 & - & 1.38 & 3.59 & 5.01 & 5.54 & 4.91 & - & 0.479 & 1098.6 & 4.89 \\
\hline 837.5 & 937.6 & 1275.6 & 1246.1 & 1232.5 & - & 1.87 & 2.41 & 2.48 & 3.02 & 3.15 & - & 0.474 & 1206.5 & 2.81 \\
\hline 619.4 & 786.0 & 1190.0 & 1363.7 & - & - & 1.40 & 2.08 & 2.64 & 4.08 & $3-$ & - & 0.463 & 1197.0 & 3.19 \\
\hline- & 576.9 & 685.4 & 1142.3 & 1228.1 & $1-$ & - & 1.11 & 1.83 & 2.93 & 4.06 & - & 0.486 & 1009.1 & 2.90 \\
\hline- & 812.8 & 943.4 & 1074.0 & 919.5 & - & - & 3.12 & 2.87 & 2.62 & - & - & 0.428 & 956.2 & 2.80 \\
\hline- & 742.1 & 641.3 & 1102.4 & 1192.4 & - & - & - & 2.64 & 3.94 & - & - & 0.446 & 995.0 & 3.40 \\
\hline- & 651.0 & 886.7 & 1148.5 & 1168.6 & - & - & - & 3.37 & 2.97 & 4.57 & - & 0.453 & 1039.3 & 4.08 \\
\hline- & 872.0 & 872.1 & 943.3 & 1112.6 & - & - & 2.72 & 3.00 & 2.77 & 2.60 & - & 0.463 & 983.0 & 2.75 \\
\hline 466.9 & 813.6 & 970.8 & 1040.7 & 1183.9 & - & 1.16 & 2.95 & 2.43 & 3.35 & 4.26 & - & 0.452 & 1028.1 & 3.36 \\
\hline 430.0 & 509.8 & \begin{tabular}{l|l}
3 & 534.6
\end{tabular} & 617.9 & - & - & 1.54 & 1.69 & 2.56 & 2.74 & - & - & 0.502 & 533.7 & 2.21 \\
\hline 209.7 & 658.7 & \begin{tabular}{|l|l}
570.1 \\
\end{tabular} & - & - & - & 1.44 & 2.81 & 2.84 & - & - & - & 0.512 & 594.5 & 2.76 \\
\hline
\end{tabular}

nicht gemessen werden konnte, hat man sie bei allon als gleich vorauggesetzt. Die Zahlen der letzten drei und Festigkeit mit der ihmen aus der Reiho der ungeraden Zahlen entsprechenden Ziffer multiplicirt, die erhalFällen, wo dio Angabo für 1 oder 1 und 2 mangeln sollte, hat man als Dirisor das Quadrat der lefzten Orinturigmittleren Schichten mangelte, so hat man für dio fehlendo Zahl das Mittel der ben.len benachbarten genommen. 
Tabelle

Zahl der Jahrringe die zwischen 2 concentrischen Kreisen, innerhalb dieser Kreise

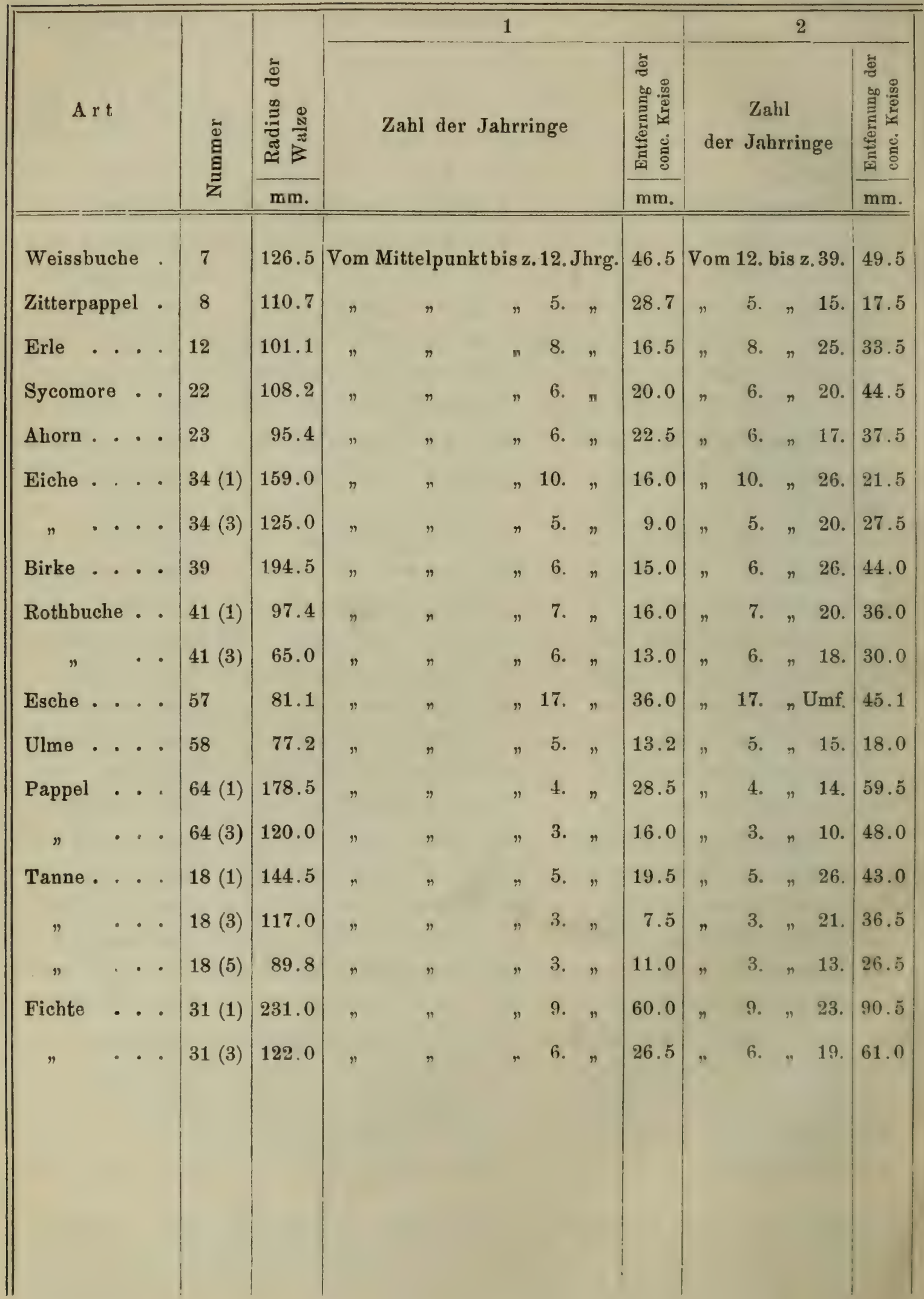


Nr. XIII.

deren die Stäbe genommen wurden, enthalten sind und Entfernung von einander.

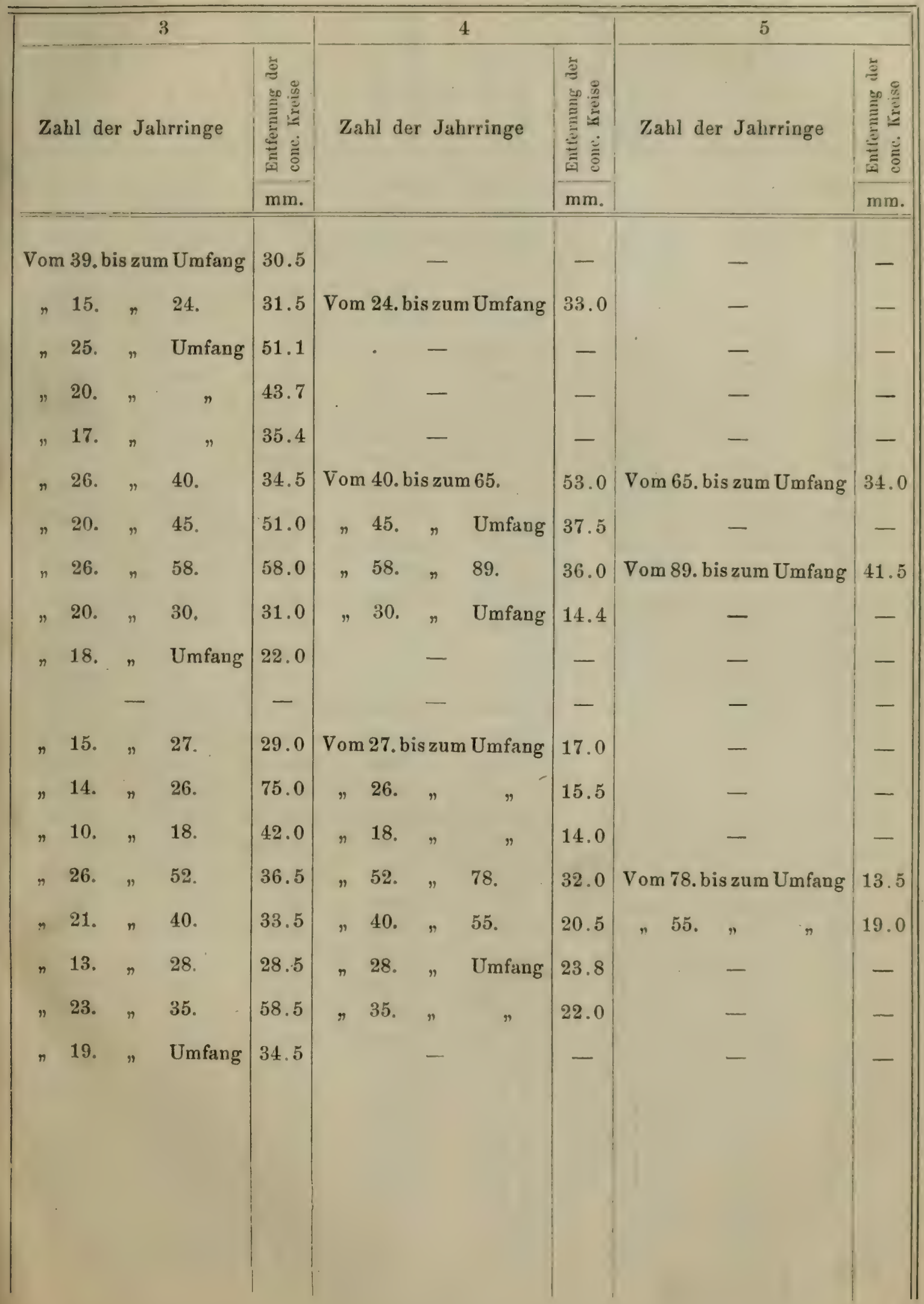


Versuche über die Biegung der ganzen Trumme.

\begin{tabular}{|c|c|c|c|c|c|c|c|c|c|c|c|}
\hline & & $r t$ & & & & 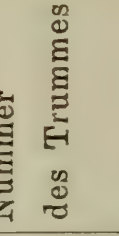 & 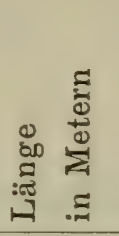 & 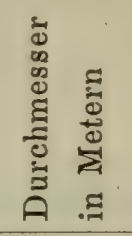 & 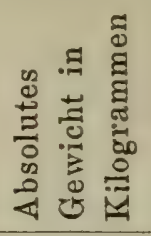 & 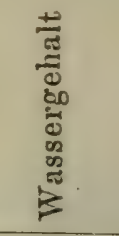 & 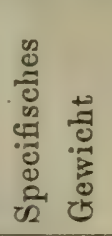 \\
\hline Rothbu & che & & . • & . . & & 1 & 2.058 & 0.4113 & 258.90 & 38.30 & 0.947 \\
\hline$\eta$ & & & . . & . . & & 6 & 2.031 & 0.2870 & 121.10 & 42.37 & 0.922 \\
\hline$"$ & & & . . & . $\cdot$ & & 11 & 2.060 & 0.2427 & 93.40 & 43.17 & 0.980 \\
\hline$"$ & & & . . & $\cdots$ & & 27 & 2.033 & 0.2513 & 90.30 & 37.42 & 0.895 \\
\hline$"$ & & & . . & . & & 29 & 2.028 & 0.3695 & 192.70 & 39.43 & 0.886 \\
\hline$\eta$ & & & - . & . . & & 32 & 2.036 & 0.4098 & 260.90 & 38.21 & 0.972 \\
\hline$\eta$ & & & . . & . . & & 33 & 2.042 & 0.2284 & 74.80 & 39.88 & 0.894 \\
\hline$"$ & & & . . & . . & & $41(2)$ & 2.061 & 0.1771 & 47.50 & 39.38 & 0.936 \\
\hline$"$ & & & . . & . . & & 42 & 2.013 & 0.1432 & 28.00 & 33.45 & 0.864 \\
\hline r & & & . . & $\cdots$ & & 44 & 2.058 & 0.1681 & 43.25 & 42.58 & 0.947 \\
\hline n & & & - . & . . & & S.-O. & 2.045 & 0.1969 & 57.20 & 34.35 & 0.919 \\
\hline$"$ & & & - . & . . & 46 & N.-O. & 2.054 & 0.1964 & 59.00 & 33.07 & 0.948 \\
\hline$n$ & & & . . & . . & & N.-W. & 2.061 & 0.1960 & 58.60 & 34.87 & 0.942 \\
\hline$"$ & & & . & $\cdots$ & & 49 & 2.039 & 0.1649 & 38.18 & 37.42 & 0.877 \\
\hline$"$ & & & . . & . . & & 53 & 2.030 & 0.2456 & 94.20 & 39.10 & 0.979 \\
\hline$\eta$ & & & . & $\cdots$ & & 55 & 2.066 & 0.2409 & 91.30 & 45.18 & 0.970 \\
\hline$"$ & & & - . & $\cdots$ & & 60 & 2.063 & 0.3111 & 138.10 & 37.15 & 0.881 \\
\hline$"$ & & & . . & $\cdots$ & & 62 & 2.075 & 0.2712 & 108.70 & 35.36 & 0.907 \\
\hline Eiche & $\cdot \cdot$ & - & . . & $\cdots$ & & 2 & 2.077 & 0.2582 & 102.70 & 38.01 & 0.944 \\
\hline n & •. & . & - . & $\cdots$ & & 3 & 2.036 & 0.2109 & 68.50 & 33.72 & 0.963 \\
\hline$"$ & . . & . & . . & . . & & 4 & 2.066 & 0.2577 & 104.50 & 36.29 & 0.970 \\
\hline$"$ & . & . & . & $\cdots$ & & 5 & 2.054 & 0.2525 & 113.20 & 52.11 & 1.101 \\
\hline$"$ & . $\cdot$ & . & . . & $\cdots$ & & 9 & 2.088 & 0.2507 & 111.80 & 55.89 & 1.085 \\
\hline$"$ & $\cdot$ & . & . . & $\cdots$ & & 10 & 2.065 & 0.1892 & 56.80 & 37.69 & 0.978 \\
\hline$n$ & $\cdot \cdot$ & . & . & $\cdots$ & & 26 & 2.009 & 0.1754 & 46.50 & 34.97 & 0.958 \\
\hline$"$ & . & . & . & $\cdots$ & & 30 & 2.024 & 0.3252 & 160.20 & 37.52 & 0.953 \\
\hline$n$ & • $\cdot$ & . & . . & . . & & $34(2)$ & 2.077 & 0.2881 & 129.20 & 40.21 & 0.954 \\
\hline$n$ & . & - & . & $\cdots$ & & $34(4)$ & 2.055 & 0.1968 & 58.80 & 40.03 & 0.941 \\
\hline$n$ & - $\cdot$ & . & - . & $\cdots$ & & 35 & 2.025 & 0.1986 & 59.40 & 38.45 & 0.947 \\
\hline$"$ & . . & . & . & $\cdots$ & & 36 & 2.030 & 0.1119 & 18.56 & 34.23 & 0.930 \\
\hline$"$ & . & • & . . & $\cdots$ & & 37 & 2.022 & 0.3547 & 182.90 & 41.70 & 0.915 \\
\hline$"$ & . & . & • · & . . & & 38 & 2.033 & 0.2499 & 111.40 & 53.75 & 1.117 \\
\hline$n$ & . $\cdot$ & . & - . & $\cdots$ & & 43 & 2.066 & 0.2790 & 128.70 & 37.11 & 1.019 \\
\hline$n$ & . & . & . . & $\cdot$ & & N.-W. & 2.029 & 0.1220 & 24.60 & 44.64 & 1.037 \\
\hline$"$ & - . & . & . $\cdot$ & $\cdots$ & 45 & S.-O. & 2.036 & 0.1224 & 24.70 & 40.69 & 1.031 \\
\hline$"$ & . . & . & . . & . & 45 & N.-O. & 2.049 & 0.1222 & 23.90 & 41.72 & 0.995 \\
\hline$"$ & . . & • & - . & $\cdots$ & & S.-W. & 2.019 & 0.1224 & 23.30 & 40.60 & 0.981 \\
\hline$"$ & . $\cdot$ & . & , . & . & & 48 & 2.053 & 0.1865 & 55.20 & 41.01 & 0.984 \\
\hline$"$ & $\cdot \cdot$ & . & • . & . & & 52 & 2.066 & 0.1725 & 43.19 & 35.03 & 0.894 \\
\hline$"$ & $\cdot \cdot$ & - & & $\cdot \cdot$ & & 56 & 2.042 & 0.1931 & 53.10 & 42.46 & 0.888 \\
\hline
\end{tabular}


Nr. XIV.

Entfernung der Stützpunkto 1.924 Meter.

\begin{tabular}{|c|c|c|c|c|c|c|c|}
\hline \multirow{2}{*}{ 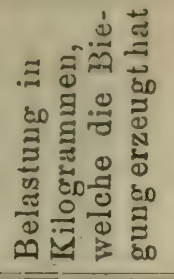 } & \multicolumn{3}{|c|}{ Pfeilhöhe in Millimeter } & \multirow{2}{*}{ 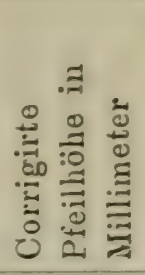 } & \multirow{2}{*}{ 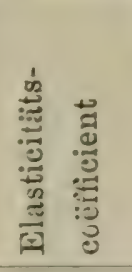 } & \multicolumn{2}{|c|}{ Bei $20 \% /$ Fenchtigkeit } \\
\hline & 1 & 2 & Mittelzahl & & & 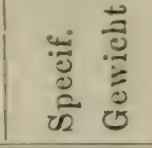 & 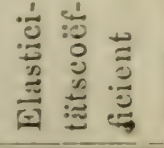 \\
\hline 10500 & 4.18 & 3.99 & 4.085 & 1.185 & 936.0 & 0.863 & 1218.7 \\
\hline 9000 & 8.13 & 7.72 & 7.925 & 5.439 & 737.3 & 0.821 & 1008.5 \\
\hline 6000 & 12.12 & 11.62 & 11.870 & 10.213 & 511.8 & 0.870 & 706.9 \\
\hline 6000 & 6.37 & 6.36 & 6.365 & 4.708 & 965.9 & 0.820 & 1243.7 \\
\hline 10500 & 4.60 & 5.19 & 4.895 & 1.995 & 853.6 & 0.802 & 1127.0 \\
\hline 10500 & 3.65 & 3.56 & 3.605 & 0.705 & - & - & - \\
\hline 6000 & 11.39 & 11.12 & 11.255 & 9.598 & 694.4 & 0.898 & 921.9 \\
\hline 3000 & 15.68 & 15.44 & 15.560 & 14.731 & 625.8 & 0.847 & 825.8 \\
\hline 1030.5 & 10.34 & 9.72 & 10.030 & 9.744 & 760.3 & 0.807 & 929.4 \\
\hline 2000 & 8.14 & 8.50 & 8.320 & 7.768 & 974.7 & 0.843 & 1336.8 \\
\hline \multirow{3}{*}{6500} & 20.94 & 20.69 & 20.815 & 19.020 & 687.3 & 0.855 & 850.4 \\
\hline & 23.88 & 23.90 & 23.895 & 22.100 & 597.6 & 0.888 & 726.8 \\
\hline & 24.84 & 25.01 & 24.925 & 23.130 & 575.6 & 0.874 & 717.1 \\
\hline 2000 & 8.81 & - & 8.810 & 8.258 & 990.2 & 0.803 & 1274.9 \\
\hline 6000 & 8.37 & 8.18 & 8.275 & 6.618 & 753.2 & 0.888 & 989.7 \\
\hline 6000 & 9.53 & 8.75 & 8.990 & 7.333 & 734.4 & 0.851 & 1037.8 \\
\hline 10500 & 8.72 & 8.38 & 8.550 & 5.650 & 599.7 & 0.807 & 769.6 \\
\hline 9000 & 8.06 & 7.63 & 7.845 & 5. 359 & 908.5 & 0.839 & 1176.7 \\
\hline 9000 & 10.33 & 10.68 & 10.505 & 8.019 & 763.3 & 0.873 & 925.6 \\
\hline 6000 & 14.59 & 14.88 & 14.735 & 13.078 & 701.0 & 0.908 & 816.4 \\
\hline 8000 & 9.84 & 9.92 & 9.880 & 7.670 & 714.9 & 0.904 & 852.2 \\
\hline 8000 & 10.39 & 10.40 & 10.395 & 8.185 & 726.9 & 0.953 & 996.4 \\
\hline 8000 & 11.13 & 11.16 & 11.145 & 8.935 & 685.1 & 0.921 & 964.3 \\
\hline 5000 & 11.99 & 10.57 & 11.280 & 9.899 & 1191.6 & 0.905 & 1439.6 \\
\hline 2815.5 & 12.16 & 11.59 & 11.875 & 11.099 & 810.2 & 0.897 & 953.3 \\
\hline 10500 & 5.98 & 6.24 & 6.110 & 3.210 & 884.1 & 0.883 & 1066.3 \\
\hline 9000 & 8.76 & $8: 28$ & 8.520 & 6.034 & 654.5 & 0.873 & 809.7 \\
\hline 5000 & 14.71 & 14.58 & 14.645 & 13.264 & 759.6 & 0.861 & 938.2 \\
\hline 4000 & 11.01 & 11.16 & 11.085 & 9.980 & 778.8 & 0.873 & 946.9 \\
\hline 1030.5 & 22.40 & 24.29 & 23.345 & 23.059 & 861.6 & 0.874 & 1006.4 \\
\hline 10500 & 5.88 & 5.85 & 5.865 & 2.965 & 676.3 & 0.832 & 848.1 \\
\hline 8000 & 14.20 & 14.22 & 14.210 & 12.000 & 516.7 & 0.959 & 716.0 \\
\hline 9000 & 8.08 & 7.62 & 7.850 & 5.364 & 837.1 & 0.946 & 1006.3 \\
\hline \multirow{4}{*}{1000} & 17.84 & 18.02 & 17.930 & 17.654 & 772.9 & 0.930 & 898.2 \\
\hline & 19.62 & 18.83 & 19.225 & 18.949 & 710.7 & 0.941 & 960.9 \\
\hline & 19.54 & 19.87 & 19.705 & 19.429 & 697.7 & 0.903 & 875.9 \\
\hline & 21.21 & 22.68 & 21.945 & 21.669 & 621.5 & 0.896 & S82. 6 \\
\hline 3000 & 10.97 & 10.40 & 10.685 & 9.856 & 760.5 & 0.897 & 948.3 \\
\hline 3000 & 15.65 & 16.76 & 16.205 & 15.376 & 666.1 & 0.838 & 784.7 \\
\hline 5000 & 9.49 & 10.34 & 9.915 & 8.534 & 1273.9 & 0.804 & 160s.s \\
\hline
\end{tabular}




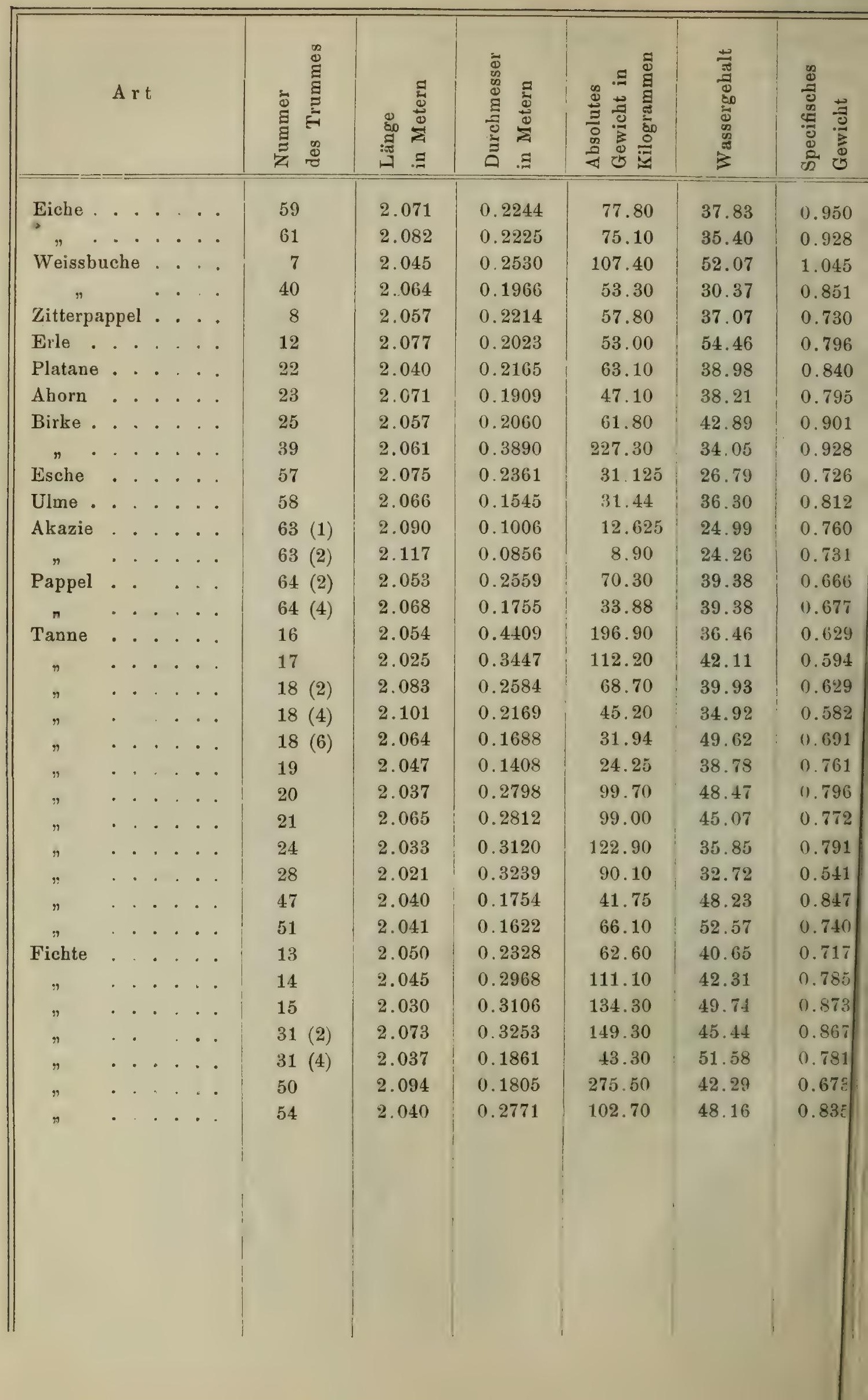




\begin{tabular}{|c|c|c|c|c|c|c|c|}
\hline \multirow{2}{*}{ 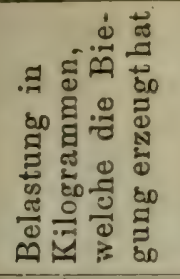 } & \multicolumn{3}{|c|}{ Pfeilhöhe in Millinetern } & \multirow{2}{*}{ 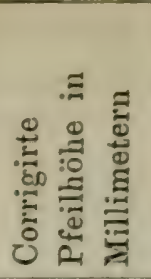 } & \multirow{2}{*}{ 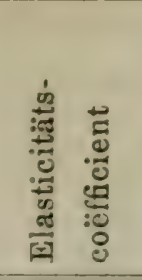 } & \multicolumn{2}{|c|}{ Bei $20 \%$ Feuchtigkeit } \\
\hline & 1 & 2 & Mittelzahl & & & 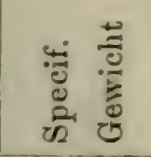 & 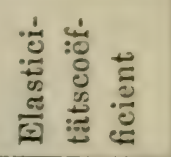 \\
\hline 6000 & 12.86 & 12.52 & 12.690 & 11.033 & 648.3 & 0.879 & 784.2 \\
\hline 6000 & 11.93 & 10.06 & 10.995 & 9.338 & 792.5 & 0.868 & 936.5 \\
\hline 8000 & 11.01 & 10.98 & 10.995 & 8.785 & 671.9 & 0.796 & 871.6 \\
\hline 5000 & 10.42 & 10.34 & 10.380 & 8.999 & 1124.3 & 0.785 & 1252.5 \\
\hline 6000 & 14.24 & 13.99 & 14.115 & 12.458 & 605.9 & 0.701 & 752.6 \\
\hline 4000 & 10.44 & 10.81 & 10.625 & 9.520 & 758.3 & 0.683 & 1115.9 \\
\hline 6000 & - & - & 9.840 & 8.183 & 1008.8 & 0.773 & 1127.8 \\
\hline 3000 & 9.39 & 8.94 & 9.165 & 8.336 & 819.1 & 0.742 & 1045.7 \\
\hline 4000 & 10.98 & 11.03 & 11.005 & 9.900 & 678.2 & 0.814 & 905.7 \\
\hline 10500 & 4.56 & 4.48 & 4.520 & 1.620 & 855.7 & 0.873 & 1032.3 \\
\hline 2000 & 9.57 & 9.66 & 9.615 & 9.063 & 963.8 & 0.701 & 993.8 \\
\hline 2000 & 11.09 & 11.69 & 11.390 & 10.838 & 979.0 & 0.761 & 1242.9 \\
\hline - & 4.95 & 4.97 & 4.960 & 4.909 & 1109.3 & 0.739 & 1141.4 \\
\hline 184.5 & 10.07 & 11.10 & 10.585 & 10.534 & 986.2 & 0.713 & 1010.7 \\
\hline 3000 & 8.90 & - & 8.900 & 8.071 & 262.0 & 0.608 & 297.2 \\
\hline 2500 & Bruch & Bruch & Bruch & Bruch & - & 0.618 & - \\
\hline 10500 & 7.41 & 7.12 & 7.265 & 4.365 & 192.4 & 0.522 & 203.9 \\
\hline 10500 & 12.68 & 12.45 & 12.565 & 9.665 & 232.6 & 0.458 & 248.3 \\
\hline 9000 & 13.54 & 13.95 & 13.745 & 11.259 & 542.0 & 0.499 & 578.0 \\
\hline 5000 & 7.69 & 7.19 & 7.440 & 6.059 & 1127.1 & 0.492 & 1193.7 \\
\hline 3000 & 14.77 & 14.94 & 14.855 & 14.026 & 796.4 & 0.480 & 814.4 \\
\hline 1030.5 & 17.41 & 16.31 & 16.860 & 16.574 & 478.0 & 0.613 & 509.1 \\
\hline 9000 & 10.08 & 9.69 & 9.885 & 7.399 & 599.9 & 0.562 & 637.3 \\
\hline 9000 & 9.87 & 10.18 & 10.025 & 7.539 & 577.1 & 0.571 & 615.4 \\
\hline 10500 & 9.69 & 9.86 & 9.775 & 6.875 & 487.2 & 0.661 & 516.7 \\
\hline 10500 & 16.97 & 17.55 & 17.260 & 14.360 & 200.8 & 0.470 & 211.6 \\
\hline 3000 & 13.74 & 13.92 & 13.830 & 13.001 & 738.7 & 0.600 & 785.2 \\
\hline 6000 & 12.25 & 12.54 & 12.395 & 10.738 & 543.6 & 0.491 & 572.2 \\
\hline 8000 & 36.20 & Bruch & 36.200 & 33.990 & 242.2 & 0.561 & 311.6 \\
\hline 10500 & 13.58 & 13.92 & 13.750 & 10.850 & 377.0 & 0.600 & 491.1 \\
\hline 10500 & 11.78 & 12.47 & 12.125 & 9.225 & 369.7 & 0.599 & 502.0 \\
\hline 10500 & 14.91 & 15.03 & 14.970 & 12.070 & 234.8 & 0.634 & 312.2 \\
\hline 5000 & $\mathrm{D}$ e $\mathrm{r}$ & $\mathrm{B}$ a $\mathrm{um}$ & $\mathrm{b} \theta \mathrm{k} a \mathrm{~m}$ & is se & - & 0.521 & - \\
\hline 10500 & - & - & 一 & - & 227.7 & 0.515 & 296.6 \\
\hline 9000 & 5.04 & 5.26 & 5.150 & 2.250 & 238.6 & 0.586 & 321.8 \\
\hline & 21.69 & 21.96 & 21.825 & 19.339 & & & \\
\hline
\end{tabular}


Nr. XV.

Festigkeit im Sinne von auf der Faser senkrechten Richtungen.

\begin{tabular}{|c|c|c|c|c|c|c|c|c|c|c|c|c|c|}
\hline & & \multirow{3}{*}{ 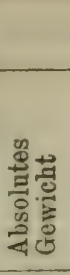 } & \multicolumn{11}{|c|}{ Im Sinne der Tangente zu den Jahrringen } \\
\hline \multirow{3}{*}{ 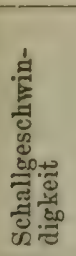 } & \multirow{3}{*}{ 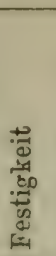 } & & \multirow{3}{*}{ 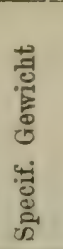 } & \multirow{2}{*}{ 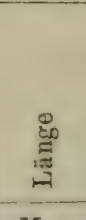 } & \multirow{2}{*}{$\begin{array}{l}\stackrel{0}{0} \\
\text { 芴 } \\
\end{array}$} & \multirow{2}{*}{$\begin{array}{l}\stackrel{8}{0} \\
\ddot{0}\end{array}$} & \multicolumn{2}{|c|}{$\begin{array}{l}\text { Zahl } \\
\text { der transversal. } \\
\text { Doppel- } \\
\text { schwingungen } \\
\text { im Sinne }\end{array}$} & \multicolumn{2}{|c|}{$\begin{array}{c}\text { Elastici- } \\
\text { tātscoerficient } \\
\text { im Sinne } \\
\end{array}$} & \multirow{3}{*}{ 芯 } & \multirow{3}{*}{ 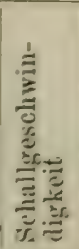 } & \multirow{3}{*}{ 竎 } \\
\hline & & & & & & & \multirow{2}{*}{ 竞 } & \multirow{2}{*}{ 吾 } & \multirow{2}{*}{ 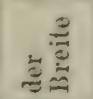 } & \multirow{2}{*}{ 竎 } & & & \\
\hline & & Gr. & & $\mathrm{Mm}$. & $\mathrm{Mm}$. & $\mathrm{Mm}$ & & & & & & & \\
\hline
\end{tabular}

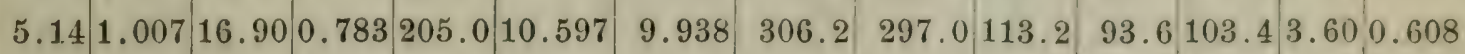
\begin{tabular}{|l|l|l|l|l|l|l|l|l|l|l|l|l|l|l|l|}
4.86 & 0.171 & 11.55 & 0.537 & 196.0 & 10.642 & 10.224 & 252.5 & 256.0 & 41.4 & 46.1 & 43.7 .2 .74 & 0.414
\end{tabular} \begin{tabular}{l|l|l|l|l|l|l|l|l|l|l|l|l|l|l|l|l|l|}
4.12 & 0.329 & 11.31 & 0.600 & 174.0 & 10.605 & 10.217 & 379.8 & 362.6 & 59.9 & 58.9 & 59.4 & 3.14 & 0.175
\end{tabular} \begin{tabular}{l|l|l|l|l|l|l|l|l|l|l|l|l|l|l|}
4.51 & 0.522 & 13.32 & 0.694 & 183.0 & 10.529 & 9.958 & 365.7 & 354.6 & 78.6 & 82.5 & 80.5 & 3.42 & 0.610
\end{tabular}

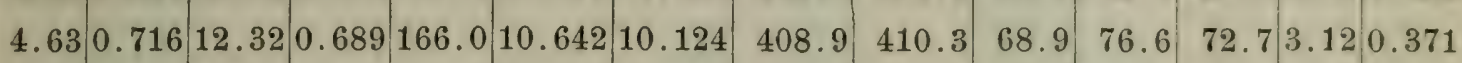

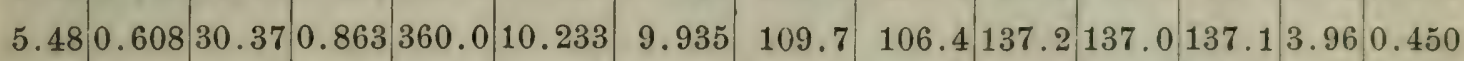

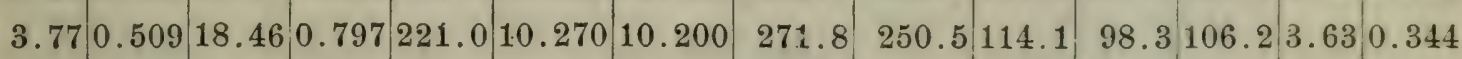

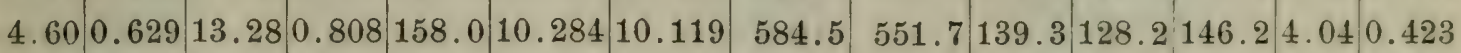
\begin{tabular}{l|l|l|l|l|l|l|l|l|l|l|l|l|l|l|l|l|l|l|l|}
3.23 & 0.823 & 18.90 & 0.801 & 231.0 & 10.408 & 9.397 & 299.2 & 271.1 & 154.7 & 155.8 & 155.2 & 4.57 & 1.063
\end{tabular} \begin{tabular}{l|l|l|l|l|l|l|l|l|l|l|l|l|l|l|l|l|l}
5.70 & 1.216 & 22.45 & 0.802 & 261.0 & 10.677 & 10.052 & 227.4 & 223.0 & 154.0 & 167.2 & 160.6 & 4.22 & 0.969
\end{tabular} \begin{tabular}{ll|l|l|l|l|l|l|l|l|l|l|l|l|l|l|l|l|l|l|}
5.53 & 0.649 & 14.22 & 0.794 & 171.0 & 10.526 & 9.949 & 528.9 & 518.2 & 156.6 & 168.2 & 162.4 & 4.26 & 0.611
\end{tabular} \begin{tabular}{l|l|l|l|l|l|l|l|l|l|l|l|l|l|l|l|l|l}
5.36 & 0.791 & 12.02 & 0.770 & 146.5 & 10.438 & 10.206 & 731.4 & 695.7 & 159.1 & 150.5 & 154.8 & 4.31 & 0.676
\end{tabular}

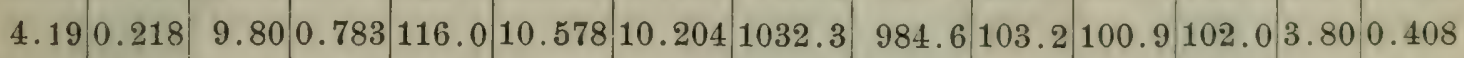
\begin{tabular}{l|l|l|l|l|l|l|l|l|l|l|l|l|l|l|}
4.28 & 0.345 & 9.51 & 0.709 & 126.0 & 10.702 & 9.953 & 699.4 & 666.7 & 61.8 & 65.0 & 63.4 & 3.05 & 0.366
\end{tabular}

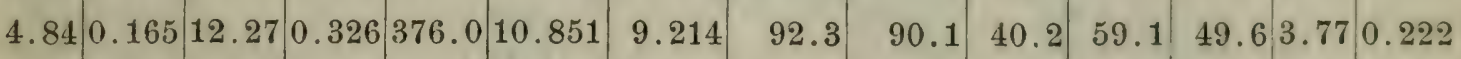
\begin{tabular}{l|l|l|l|l|l|l|l|l|l|l|l|l|l|l|l|l|l|l|l|}
4.07 & 0.128 & 9.55 & 0.349 & 231.0 & 11.034 & 10.745 & - & - & - & - & - & - & 0.202
\end{tabular}

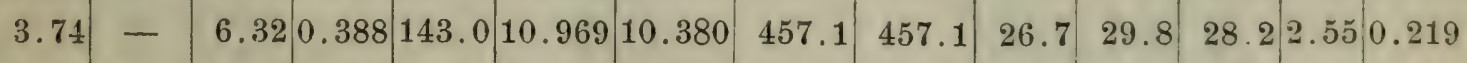

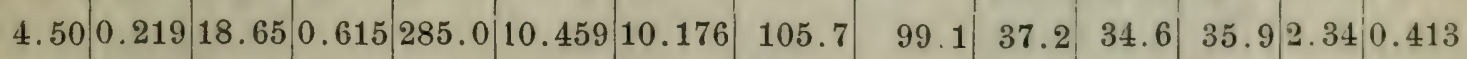

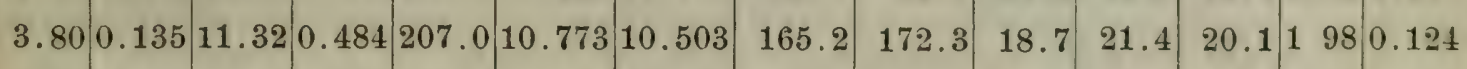

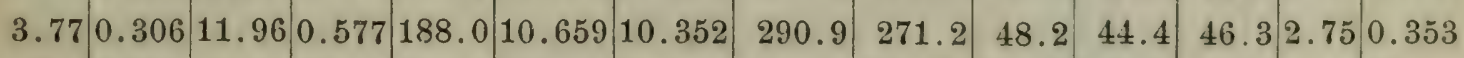

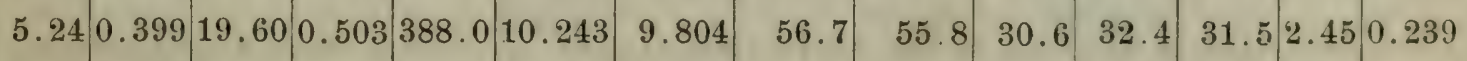

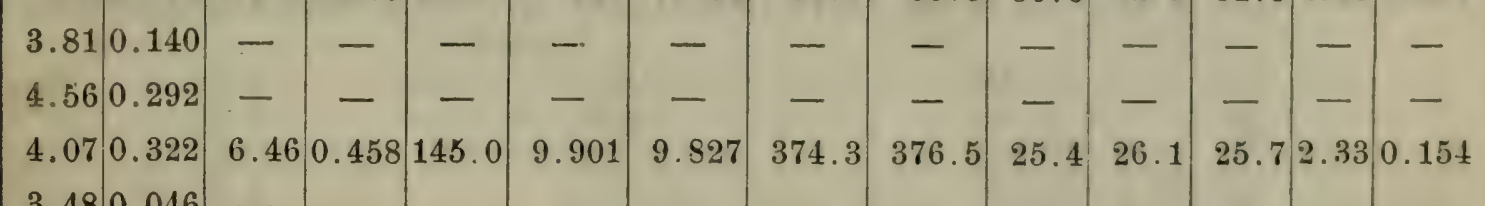
\begin{tabular}{l|l|l|l|l|l}
3.48 & 0.046 & - & - & - \\
4.44 & - & 5.60 & 0.830 & 98.4
\end{tabular} $4.44-5.600 .830 \quad 98.4$

\begin{tabular}{l|l|l|l|l|l|l|l|l}
8.103 & 8.472 & 1319.6 & 1354.5 & 164.8 & 158.8 & 161.8 & 4.16 & 1.092
\end{tabular} 6.630 .800119 .5

\begin{tabular}{l|l|l|l|l|l|l|l|l|l|l|l|l|l|l|}
8.187 & 8.467 & 864.9 & 876.7 & 145.5 & 139.8 & 142.6 & 3.98 & 1.370
\end{tabular} 
Einfluss der Höhe im Baume. Verhältniss der Elasticitätscoëfficienten

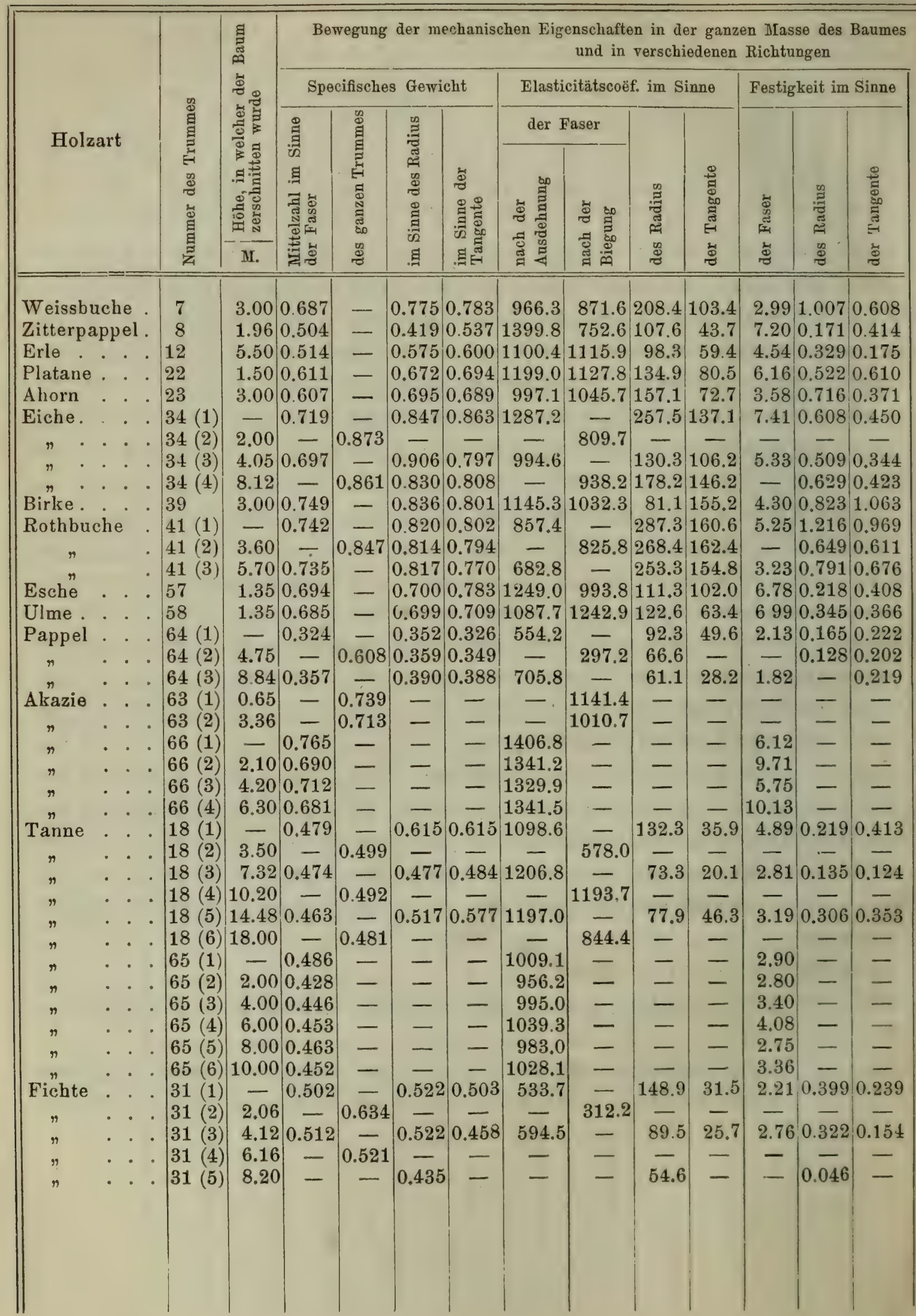


Nr. XVI.

und Festigkeiten in den drei auf einander senkrechten Richtungen.

bei verschiedenen Höhen

Bowegung der mechanischen Eigenschaften in ein und demselben Jahrringe hei verschiedener Höhe

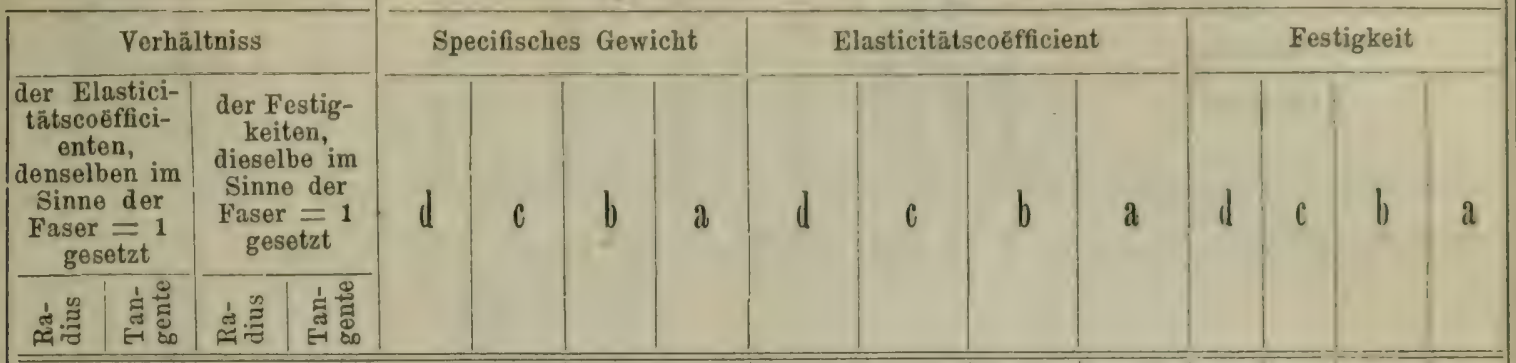

$\begin{array}{lllll}0.216 & 0.107 & 0.337 & 0.203\end{array}$ $\begin{array}{llllll}0.077 & 0.031 & 0.024 & 0.057\end{array}$ \begin{tabular}{ll|l|l|l|l|l|l|l|l|l|l|l|}
0.089 & 0.054 & 0.072 & 0.038
\end{tabular} \begin{tabular}{ll|l|l|l|l|l|l|l|l|l|l|}
0.112 & 0.067 & 0.085 & 0.099
\end{tabular}

$0.1570 .0730 .200 \quad 0.104$

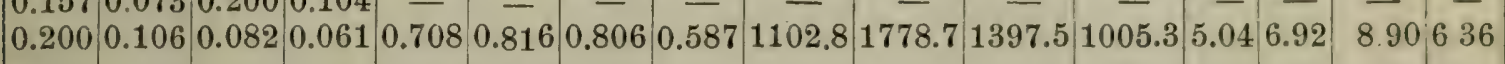

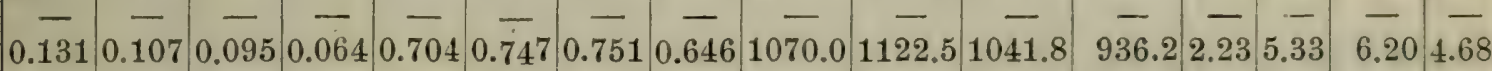
$0.1900 .156--$

$\begin{array}{lllll}0.071 & 0.135 & 0.191 & 0.247\end{array}$

\begin{tabular}{lll|l|l|l|l|l|l|l|l|l}
0.335 & 0.187 & 0.232 & 0.184 & 0.747 & 0.754 & 0.712 & -
\end{tabular} 0.3250 .197 $\begin{array}{lllllllll}0.371 & 0.227 & 0.245 & 0.209 & 0.662 & 0.824 & 0.663 & -\end{array}$ \begin{tabular}{ll|l|l|l|l|l|l|l}
0.089 & 0.082 & 0.321 & 0.602 & - & - & - & - \\
0.113 & 0.058 & 0.493 & 0.524 & - & - & - & -
\end{tabular} \begin{tabular}{ll|l|l|l|l|l|l|l|l|l|l}
0.167 & 0.090 & 0.077 & 0.104 & 0.316 & 0.330 & 0.313 & -
\end{tabular} \begin{tabular}{l|ll|l|l|l|l|l}
0.224 & - & - & - & - & - & - & - \\
0.086 & 0.040 & - & 0.120 & 0.352 & 0.347 & 0.370 & -
\end{tabular}

\begin{tabular}{lll|l|l|l|l|l}
- & - & - & - & - & - & - & - \\
- & - & - & - & $\overline{6}$ & - & - & -
\end{tabular}

\begin{tabular}{lll|l|l|l|l|l}
- & - & - & - & 0.697 & 0.741 & 0.831 & - \\
- & - & - & - & - & - & - & -
\end{tabular} \begin{tabular}{l|c|c|c|c|c|c|c}
$-\overline{120}$ & 0.033 & 0.045 & 0.084 & 0.406 & 0.471 & 0.488 & 0.528
\end{tabular} \begin{tabular}{c|c|c|c|c|c|c|c}
$0 . \overline{061}$ & $0 . \overline{016}$ & $0 . \overline{048}$ & $0 . \overline{044}$ & $0 . \overline{662}$ & $0 . \overline{482}$ & $0 . \overline{485}$ & $0 . \overline{447}$
\end{tabular}

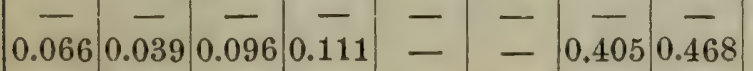
\begin{tabular}{c|c|c|c|}
- & - & - & - \\
657.6 & 897.7 & 998.4 & - \\
- & - & - & - \\
568.0 & 716.8 & 739.0 & - \\
- & - & - & - \\
- & - & - & - \\
553.4 & 569.4 & 472.7 & - \\
- & - & - & - \\
751.1 & 697.0 & 736.7 & - \\
- & - & - & - \\
- & - & - & - \\
1323.9 & 1445.4 & 1447.8 & - \\
- & - & - & - \\
- & - & - & -
\end{tabular}

\begin{tabular}{c|c|c|c}
- & - & - & - \\
\hline-21 & 6.64 & 4.24 & - \\
- & - & - & - \\
1.11 & 3.35 & 3.24 & - \\
- & - & - & - \\
- & - & - & - \\
2.15 & 2.21 & 1.64 & - \\
- & - & - & - \\
1.88 & 0.94 & 2.10 & - \\
- & - & - & - \\
- & - & - & - \\
4.61 & 5.36 & 7.82 & - \\
- & - & - & - \\
\hline 5.09 & 7.64 & 11.88 & -
\end{tabular} \begin{tabular}{r|r|r|c|r|r|r|r|r}
1030.3 & 1201.4 & 1423.9 & - & 5.09 & 7.64 & 11.88 & - \\
883.2 & 1165.5 & 1061.5 & 1286.6 & 3.59 & 5.01 & 5.54 & 4.91 \\
\hline
\end{tabular}

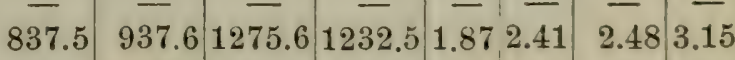

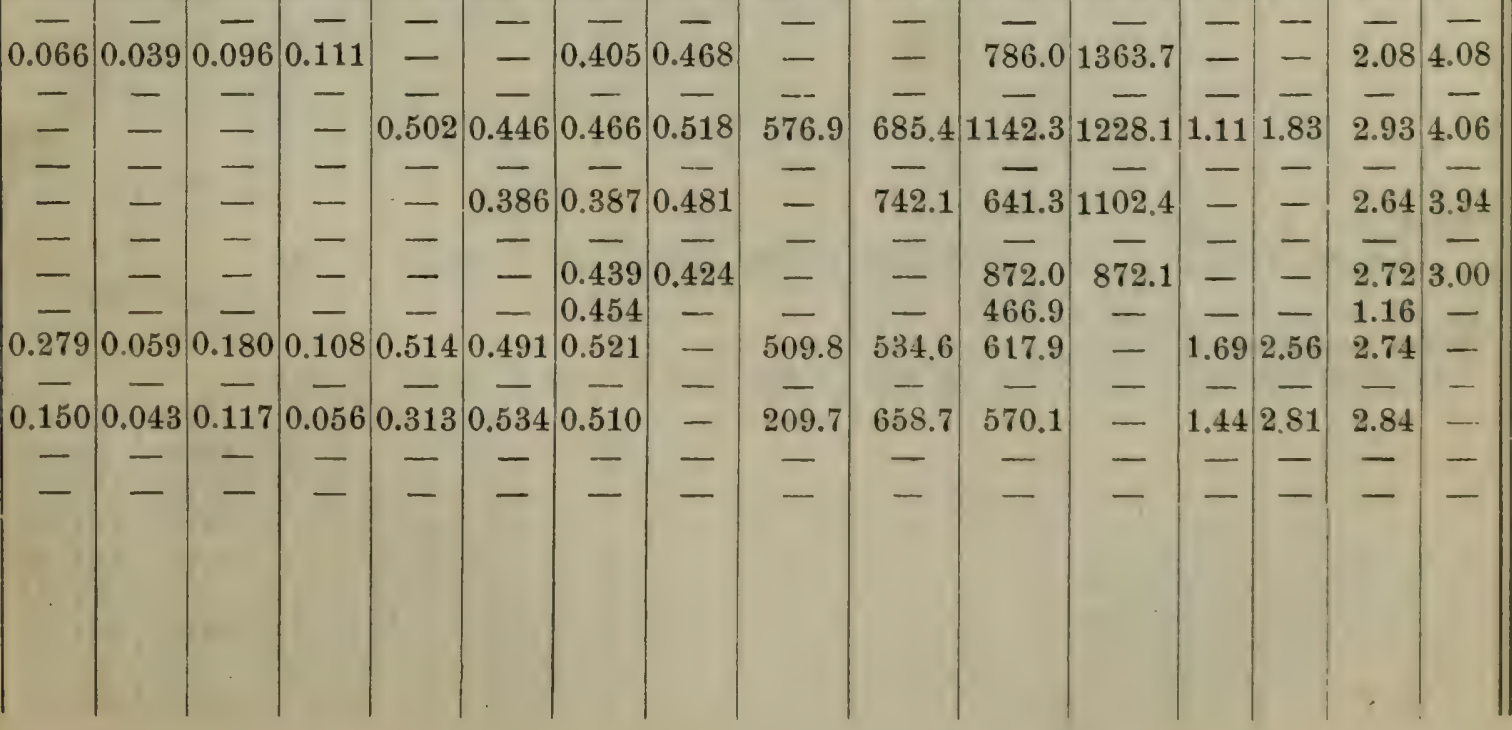


Versuche mit den im Hande1

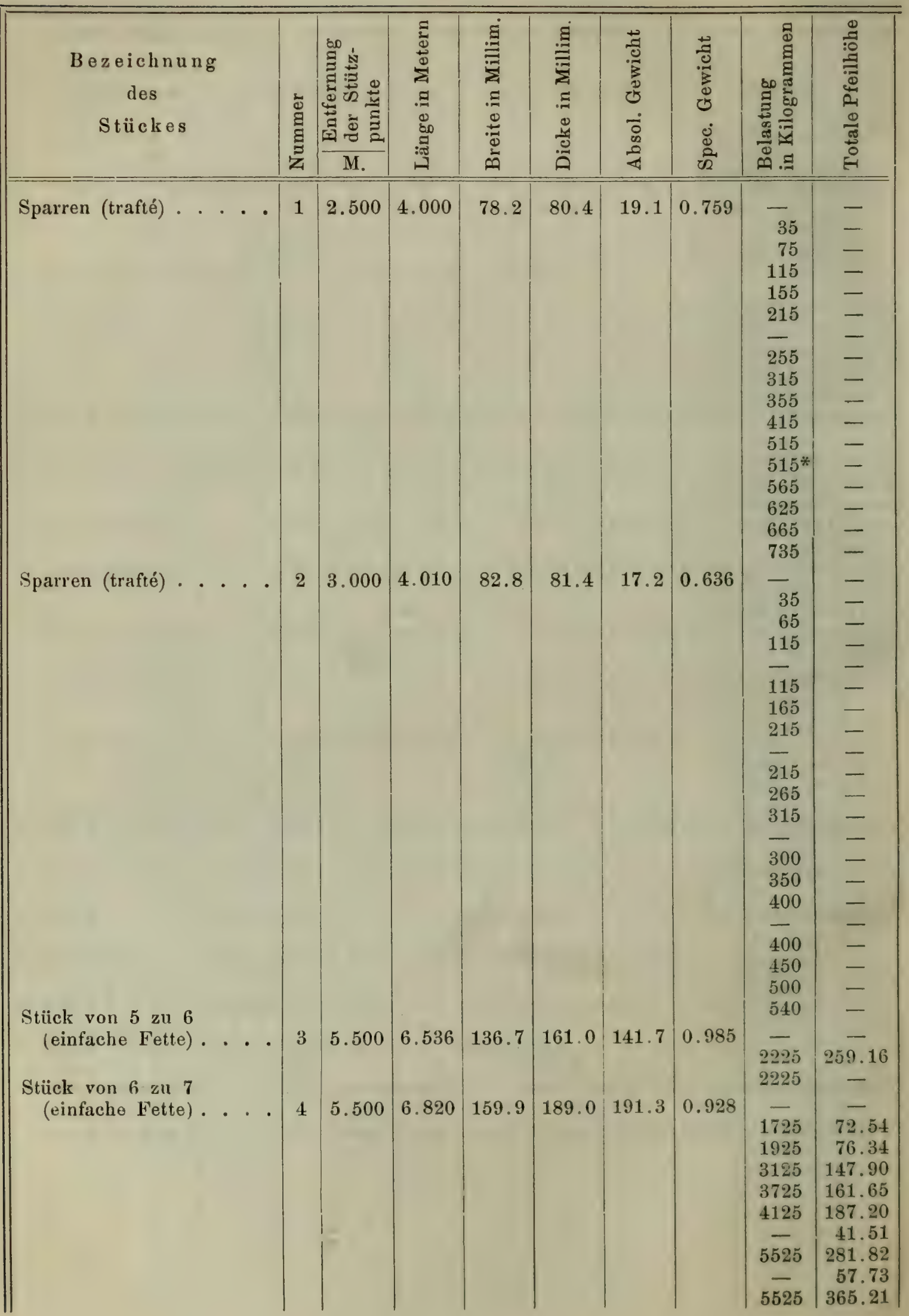


Nr. XVII.

\section{$-111$}

vorkommenden Eichenholzstücken.

\begin{tabular}{|c|c|c|c|c|c|c|c|c|}
\hline \multicolumn{2}{|c|}{ 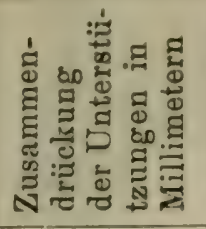 } & \multirow[t]{2}{*}{ 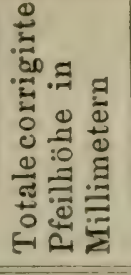 } & \multirow[t]{2}{*}{ 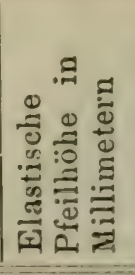 } & \multirow{2}{*}{\multicolumn{2}{|c|}{$\begin{array}{c}\text { Mittlere Pfeilhöhe } \\
\text { für eine } \\
\text { Belastung von } \\
100 \text { Kilogrammen } \\
\text { in Millim. }\end{array}$}} & \multirow[t]{2}{*}{ 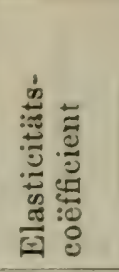 } & \multirow{2}{*}{ 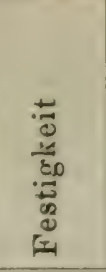 } & \multirow[t]{2}{*}{ Anmerkung } \\
\hline $\mathbf{A}$ & B & & & & & & & \\
\hline - & - & - & & & & & & \\
\hline - & - & 4.20 & 4.20 & 12.000 & & & & \\
\hline - & - & 9.42 & 9.42 & 12.560 & & & & \\
\hline - & 一 & 14.86 & 14.86 & 12.922 & & & & \\
\hline - & 一 & 19.79 & 19.79 & 12.768 & & & & \\
\hline - & 一 & 26.98 & 26.16 & 12.167 & 12.412 & 774.3 & 5.60 & \\
\hline - & $\overline{-}$ & $\begin{array}{r}0.82 \\
31.84\end{array}$ & $\overline{31.02}$ & $12 \overline{1} 65$ & & & & \\
\hline - & - & 39.96 & 39.14 & 12.425 & & & & \\
\hline - & 一 & 43.83 & 43.01 & 12.116 & & & & \\
\hline - & - & 51.61 & 50.79 & 12.239 & & & & \\
\hline- & - & 66.53 & 65.71 & 12.759 & & & & \\
\hline- & 一 & 85.19 & & & & & & $\begin{array}{l}\text { "Nachdem es derselben Belastung } \\
\text { in der Dauer von } 12 \text { Stunden unter- }\end{array}$ \\
\hline - & 一 & 90.62 & & & & & & worfen wurde. \\
\hline - & - & $\begin{array}{r}98.59 \\
105\end{array}$ & & & & & & \\
\hline - & - & $\begin{array}{c}105.20 \\
\text { Bruch }\end{array}$ & & & & & & Plötzlicher Bruch. \\
\hline - & - & - & & & & & & \\
\hline - & - & 8.56 & 8.56 & 24.457 ) & & & & \\
\hline - & - & 20.17 & 20.17 & 31.031 & & & & \\
\hline - & - & 27.85 & 27.85 & 24.217 & & & & \\
\hline - & - & - & - & - & & & & \\
\hline - & 一 & 26.05 & 26.05 & 22.600 & & & & \\
\hline - & - & 41.16 & 41.16 & 24.946 & & & & \\
\hline - & - & 49.56 & 49.86 & 23.194 & & & & \\
\hline - & - & 0.30 & - & - & & & 4.60 & \\
\hline - & - & 51.56 & 51.86 & 24.121 & 25.139 & 601.3 & & \\
\hline - & - & 64.18 & - & 24.035 & & & & \\
\hline - & - & $\begin{array}{r}77.68 \\
1.97\end{array}$ & $\begin{array}{l}75.71 \\
-\end{array}$ & $\begin{array}{c}24.035 \\
-\end{array}$ & & & & \\
\hline - & - & 77.94 & 75.97 & 25.323 & & & & \\
\hline - & - & 91.23 & - & - & & & & \\
\hline- & - & 113.38 & 105.04 & 26.260 & & & & \\
\hline - & - & 8.34 & - & - & & & & \\
\hline - & - & 113.73 & 105.39 & $2(6.347)$ & & & & \\
\hline- & - & 132.39 & & & & & & \\
\hline - & - & $\begin{array}{c}147.36 \\
\text { Bruch }\end{array}$ & & & & & & \\
\hline- & - & - & & & & & & \\
\hline 1.86 & 7.95 & 254.26 & 254.26 & 11.425 & 11.425 & 638.1 & 5.71 & \\
\hline - & - & $\begin{array}{c}\text { Bruch } \\
-\end{array}$ & & & & & & $\begin{array}{l}\text { fernung von } 50 \text { Centimetern ron der } \\
\text { Mitte an einer Aststelle. }\end{array}$ \\
\hline 1.56 & 3.93 & 69.80 & 69.80 & $4.046)$ & & & & \\
\hline 1.74 & 4.47 & 73.24 & 73.24 & 3.810 & & & & \\
\hline 3.93 & 9.93 & 140.97 & - & - & & & & \\
\hline 4.18 & 10.55 & 154.29 & - & - & 3.826 & 1007.0 & 10.55 & \\
\hline 4.85 & 11.69 & 178.93 & 144.08 & 3.493 & & & & $\begin{array}{l}\text { - Der Bruch erfolgte sehr langsam, } \\
\text { nachdern die Belastung von } 5525 \mathrm{Ki} \text { - }\end{array}$ \\
\hline 5.30 & 8.03 & 34.85 & - & - & & & & logrnmmen durch mehr als eine \\
\hline 6.16 & 20.45 & 268.52 & 218.56 & $3.955)$ & & & & Lingsrisse hatten sich gevildet und \\
\hline 6.14 & $\begin{array}{c}9.41 \\
-\end{array}$ & $\begin{array}{c}49.96 \\
\text { Bruch }\end{array}$ & & & & & & $\begin{array}{l}\text { daun trat der Brucis } 25 \text { Centimeter } \\
\text { ron der Mitte ein. }\end{array}$ \\
\hline - & - & & & & & & & \\
\hline
\end{tabular}




\begin{tabular}{|c|c|c|c|c|c|c|c|c|c|}
\hline $\begin{array}{c}\text { Bezeichnung } \\
\text { des } \\
\text { Stückes }\end{array}$ & 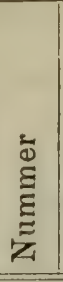 & 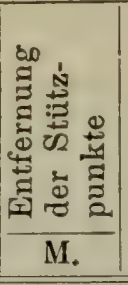 & 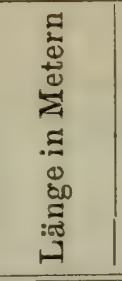 & 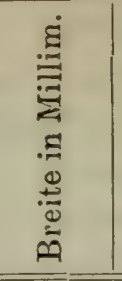 & 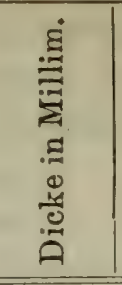 & 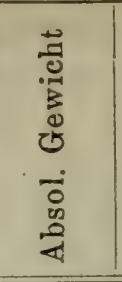 & 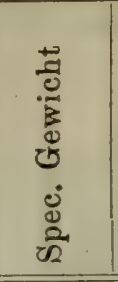 & 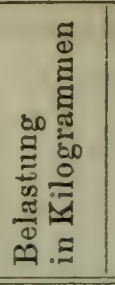 & 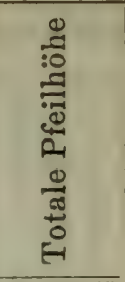 \\
\hline Stück von 7 zu 8 (Fette) & 5 & 5.500 & 6.103 & 182.1 & 209.7 & 220.0 & 0.944 & $\overline{2389}$ & Bruch \\
\hline Stiick von $7 \mathrm{zu} 8$ (Fette) & 6 & 5.500 & 7.062 & 190.7 & 220.0 & 273.2 & 0.922 & $\begin{array}{c}- \\
2725 \\
- \\
- \\
2725 \\
3225 \\
3725 \\
4225 \\
4725 \\
5125 \\
5225\end{array}$ & \begin{tabular}{r}
\multicolumn{1}{c}{$\overline{72.49}$} \\
5.64 \\
5.14 \\
71.56 \\
80.58 \\
92.12 \\
114.33 \\
149.47 \\
208.13 \\
Bruch
\end{tabular} \\
\hline Stück von 8 zu 9 (Fette) & 8 & 5.500 & 6.110 & 216.7 & 236.7 & 300.3 & 0.958 & $\begin{array}{c}- \\
2225 \\
- \\
2225 \\
2725 \\
3025 \\
3025^{*} \\
3725 \\
4525 \\
5125 \\
5725^{*} \\
6125 \\
6725 \\
7189\end{array}$ & $\begin{array}{r}\overline{43.17} \\
2.70 \\
43.44 \\
50.49 \\
57.13 \\
58.53 \\
68.64 \\
89.81 \\
103.54 \\
120.99 \\
146.67 \\
204.00 \\
\text { Bruch }\end{array}$ \\
\hline Stück von $81 / 2$ zu $91 / 2$ (Fette) & 7 & 5.500 & 5.870 & 231.8 & 252.8 & 346.7 & 1.008 & $\begin{array}{c}- \\
3925 \\
- \\
3925 \\
5525 \\
6025 \\
6725 \\
7725 \\
7889\end{array}$ & \begin{tabular}{r}
\multicolumn{1}{c}{$\overline{57.68}$} \\
5.82 \\
58.43 \\
85.18 \\
93.43 \\
113.39 \\
161.21 \\
Bruch
\end{tabular} \\
\hline Brett (échantillon).... & 10 & 3.000 & 3.652 & 143.4 & 42.2 & 18.2 & 0.824 & \begin{tabular}{r|}
- \\
35 \\
55 \\
75 \\
95 \\
115 \\
135 \\
155 \\
175 \\
195 \\
215 \\
255 \\
295 \\
375
\end{tabular} & $\begin{array}{l}- \\
= \\
- \\
- \\
- \\
- \\
- \\
- \\
- \\
-\end{array}$ \\
\hline
\end{tabular}




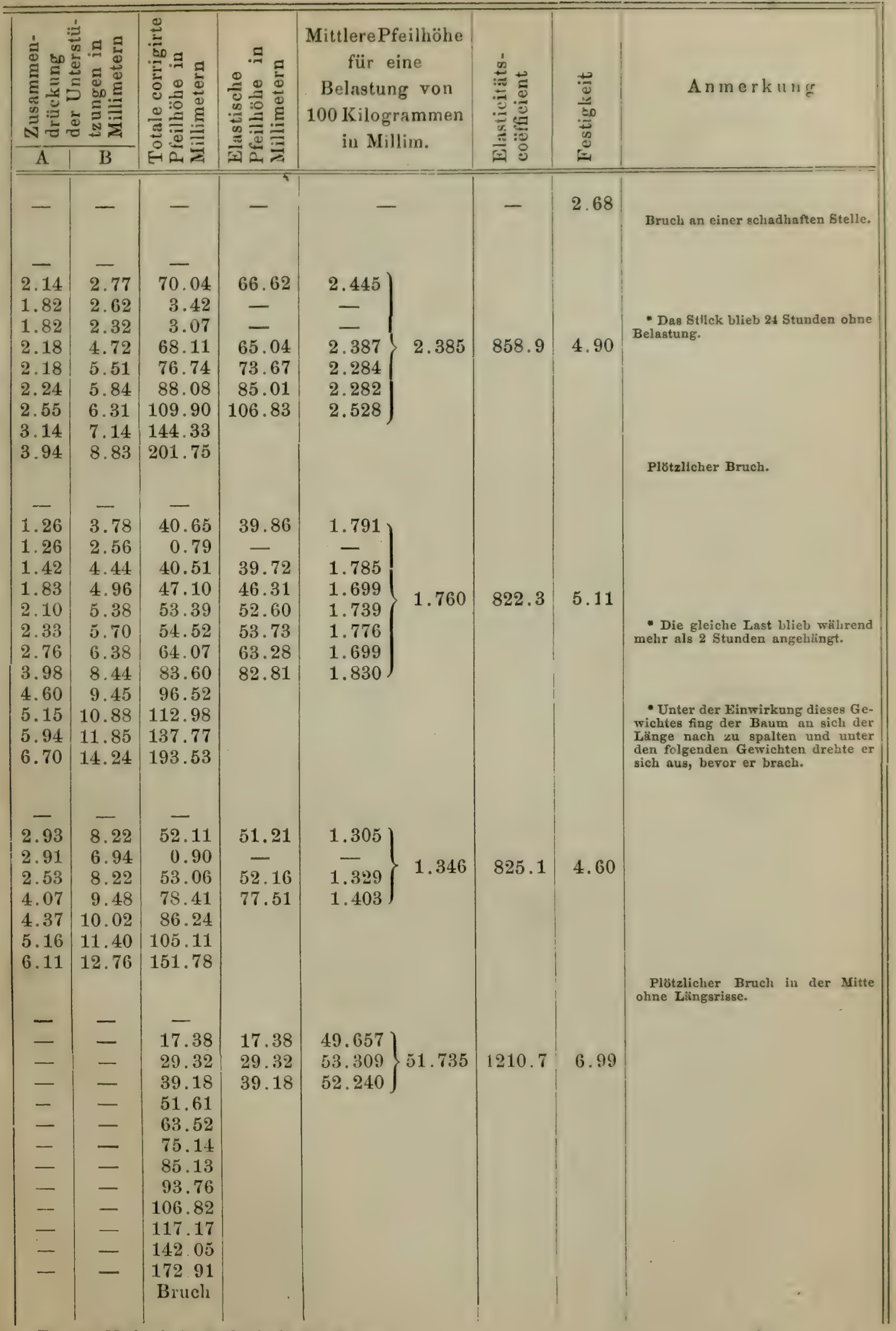

Exner. Mechanische Technologie des Holzes. 


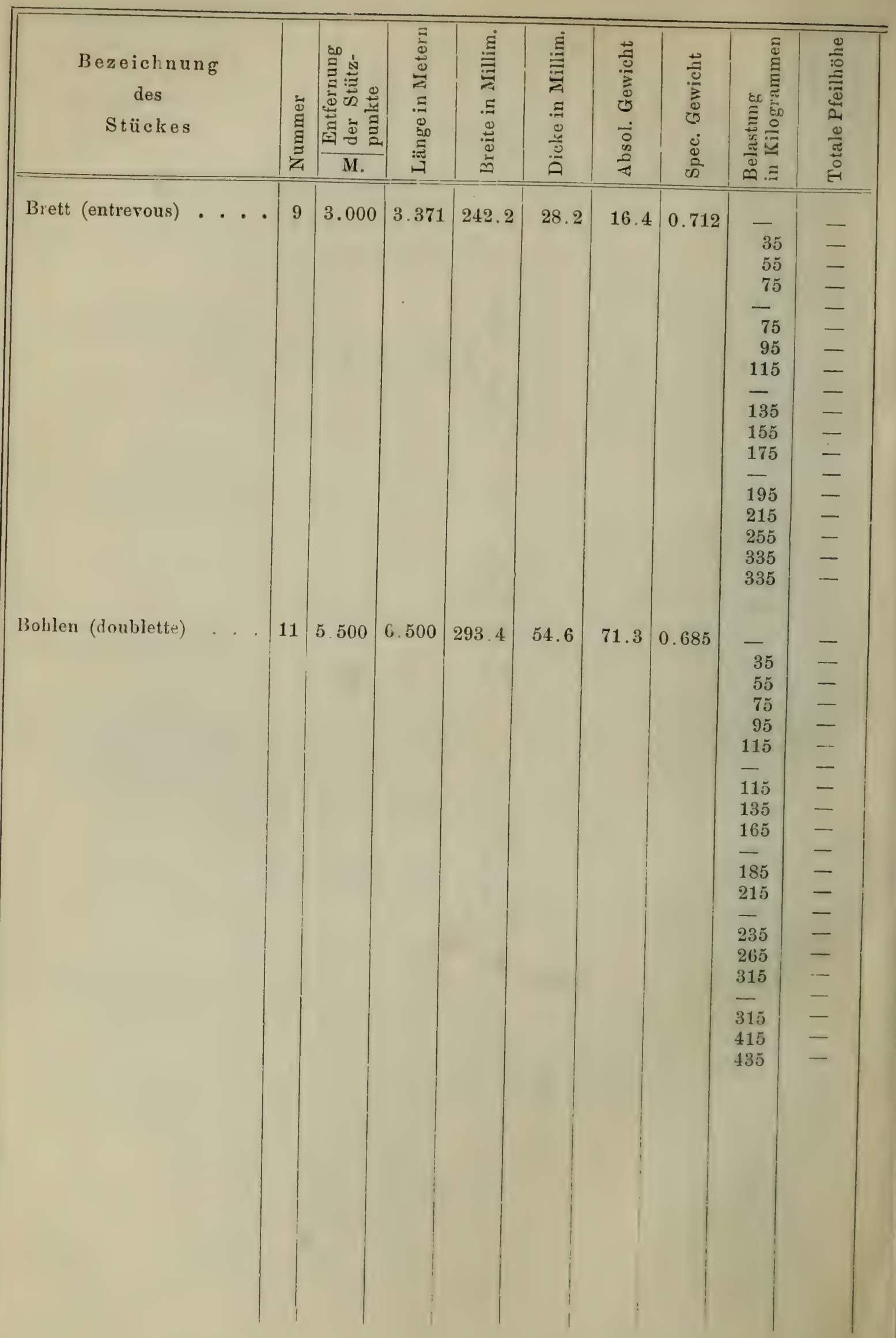




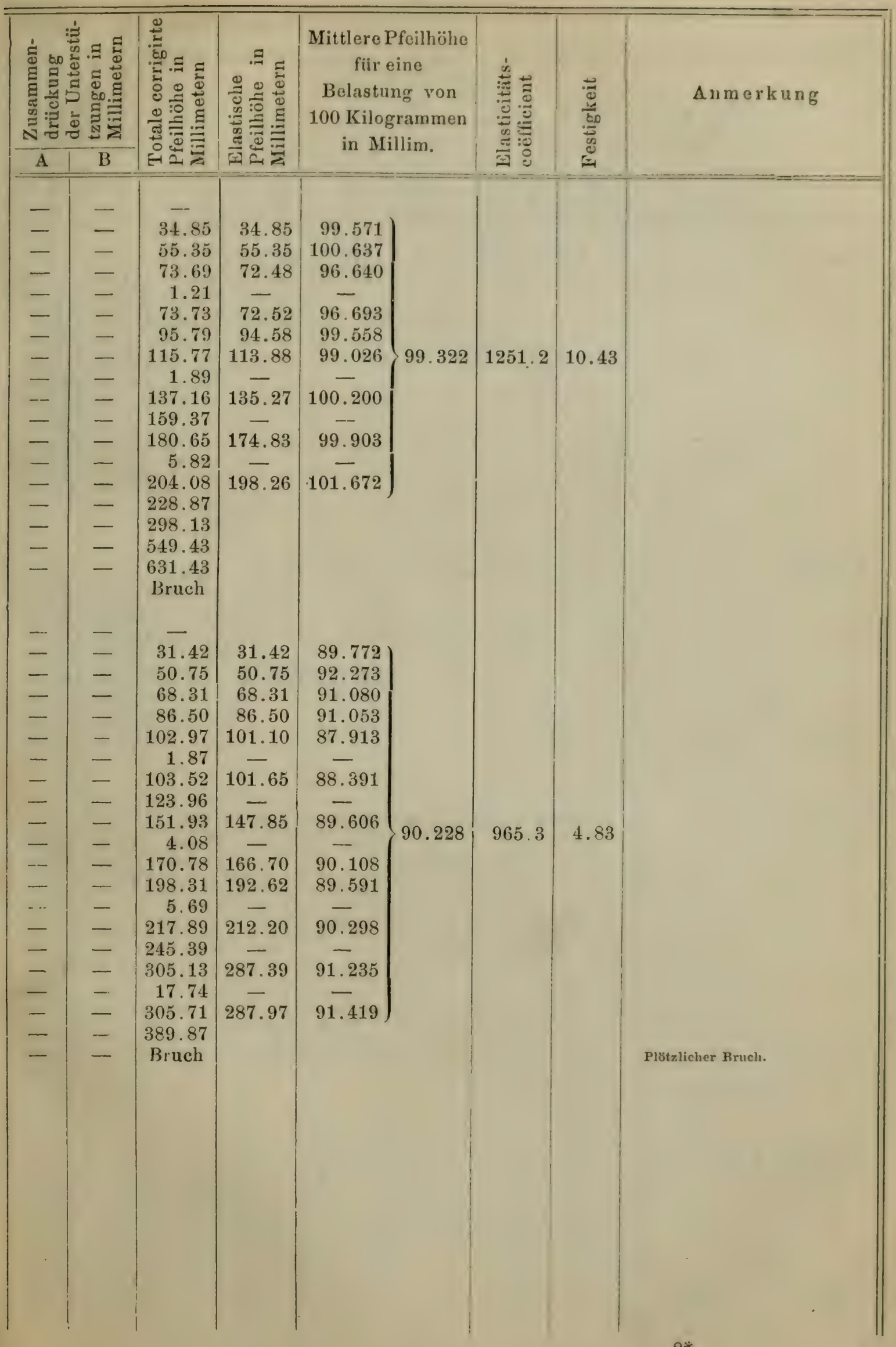


Versuche mit den im Handel

\begin{tabular}{|c|c|c|c|c|c|c|c|c|c|c|}
\hline $\begin{array}{c}\text { Bezeichnun } \\
\text { des } \\
\text { Stückes }\end{array}$ & & : & 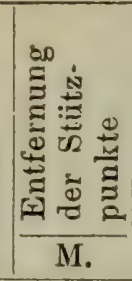 & 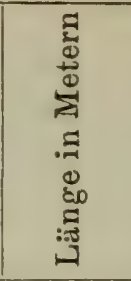 & 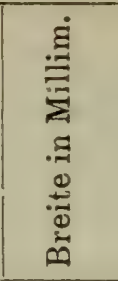 & 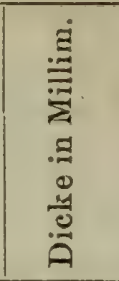 & 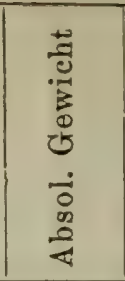 & 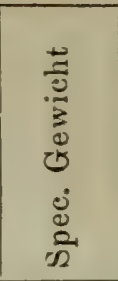 & 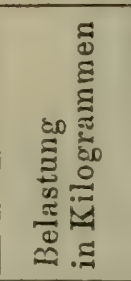 & 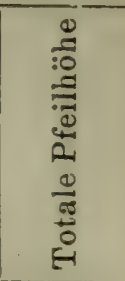 \\
\hline Sparren . . . & $\cdot \cdot$ & 2 & 9.000 & 10.360 & 87.8 & 115.0 & 50.75 & 0.485 & $\begin{array}{l}\overline{77.0} \\
\overline{117} .0 \\
\overline{6} .0 \\
\overline{6} .0 \\
21 \overline{7} .0 \\
\overline{317} .0 \\
\overline{417.0}\end{array}$ & \begin{tabular}{r}
\multicolumn{1}{c}{$\overline{94.01}$} \\
1.27 \\
146.33 \\
2.76 \\
217.39 \\
10.39 \\
284.61 \\
12.13 \\
463.55 \\
38.81 \\
Bruch
\end{tabular} \\
\hline Sparren . . . & $\cdot \cdot$ & 6 & 9.000 & 10.560 & 93.7 & 130.1 & 58.45 & 0.454 & $\begin{array}{l}\overline{1 \overline{17} .0} \\
\overline{217} .0 \\
\overline{317} .0 \\
\overline{-} \\
417.0 \\
512.0\end{array}$ & $\begin{array}{r}- \\
77.79 \\
1.18 \\
158.89 \\
3.55 \\
232.31 \\
6.29 \\
314.90 \\
\text { Bruch }\end{array}$ \\
\hline Sparren . . . & $\cdot \cdot$ & 8 & 9.000 & 10.500 & 96.5 & 124.1 & 63.25 & 0.503 & 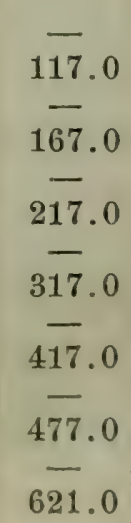 & $\begin{array}{r}- \\
83.89 \\
1.18 \\
122.15 \\
3.07 \\
157.07 \\
3.27 \\
233.10 \\
5.02 \\
308.43 \\
6.82 \\
362.88 \\
13.16 \\
\text { Bruch }\end{array}$ \\
\hline $\begin{array}{r}\text { Stück von } 6 \mathrm{zu} 7 \\
\text { (einfache Fette) }\end{array}$ & . . & 9 & 9.000 & 10.410 & 164.0 & 194.5 & 169.50 & 0.510 & $\begin{array}{r}- \\
313.0 \\
1012.8 \\
313.0 \\
1712.8 \\
313.0 \\
2512.8\end{array}$ & $\begin{array}{r}- \\
20.16 \\
110.79 \\
28.25 \\
204.66 \\
36.80 \\
\text { Bruch }\end{array}$ \\
\hline
\end{tabular}


Nr. XVIII.

\section{- 117}

vorkommenden Tannenholz-Sortimenten.

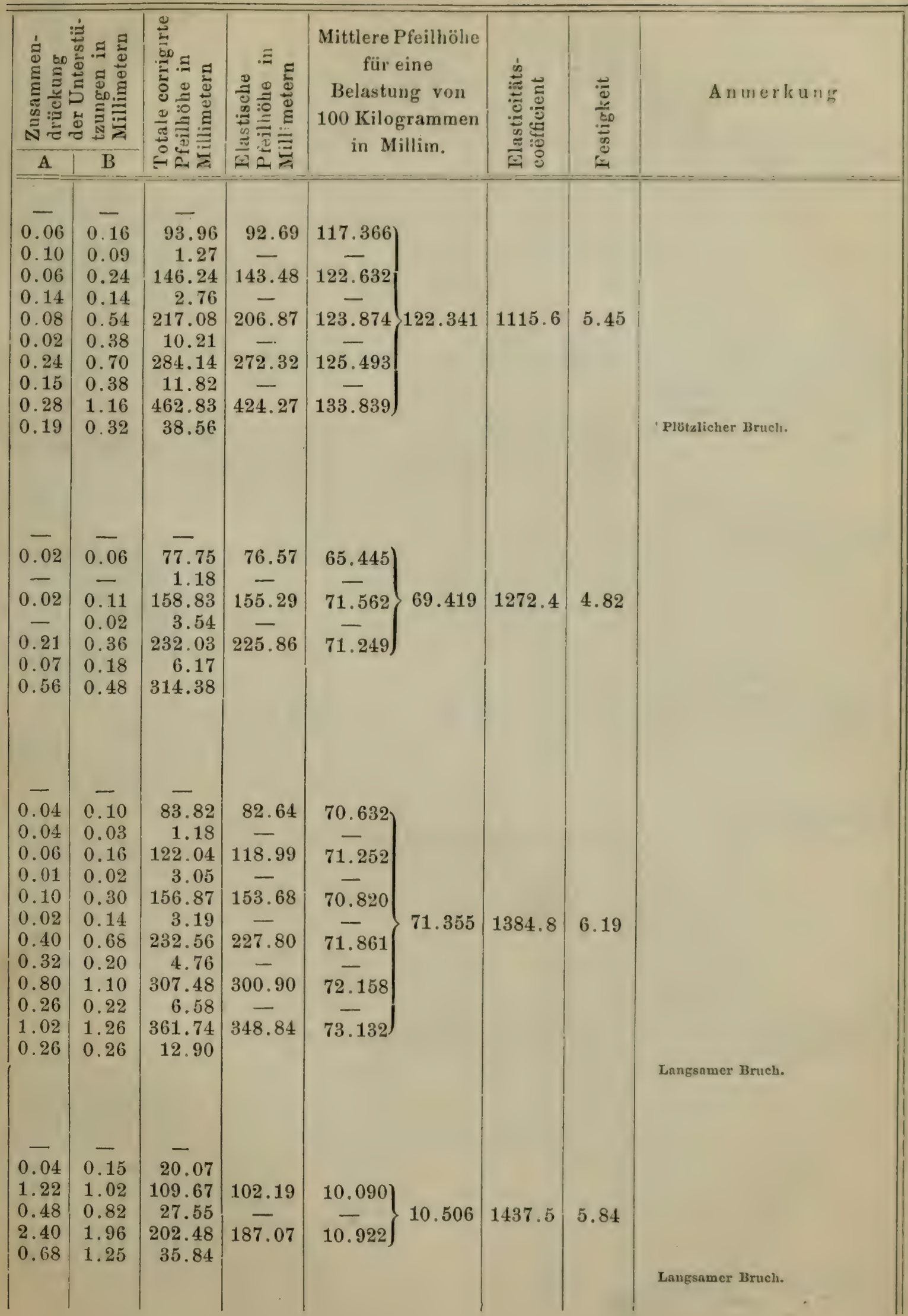




\begin{tabular}{|c|c|c|c|c|c|c|c|c|c|}
\hline $\begin{array}{c}\text { Bezeichung } \\
\text { des } \\
\text { Stiickes }\end{array}$ & 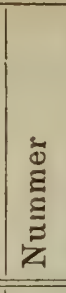 & 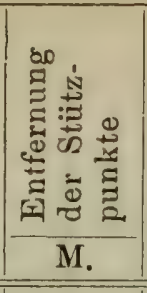 & 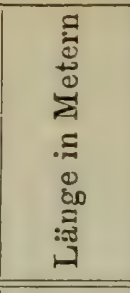 & 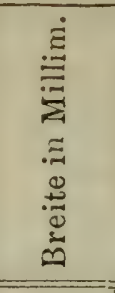 & 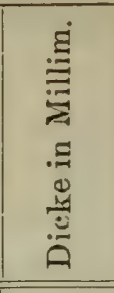 & 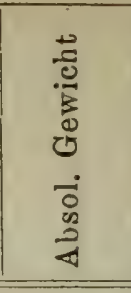 & 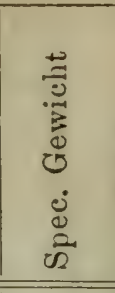 & 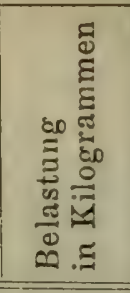 & 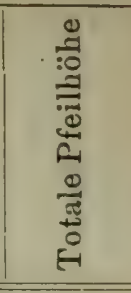 \\
\hline $\begin{array}{l}\text { Stiick von } 6 \text { zu } 7 \\
\text { (einfache Fette) }\end{array}$ & 5 & 9.000 & 10.490 & 169.2 & 196.1 & 171.00 & 0.491 & $\begin{array}{r}313.0 \\
1012.8 \\
313.0 \\
1712.8 \\
313.0 \\
1932.8\end{array}$ & $\begin{array}{r}29.99 \\
139.49 \\
41.41 \\
283.66 \\
63.40 \\
\text { Bruch }\end{array}$ \\
\hline $\begin{array}{l}\text { Stäck von } 6 \text { zu } 7 \\
\quad \text { (einfache Fette) } .\end{array}$ & 1 & 9.000 & 10.470 & 176.5 & 198.4 & 210.00 & 0.573 & $18 \overline{0} .0$ & $-\overline{B r u c h}$ \\
\hline $\begin{array}{l}\text { Stïck von } 8 \text { zu } 9 \\
\text { (Fette). } . . . . .\end{array}$ & 3 & 9.000 & 10.470 & 220.2 & 245.5 & 304.00 & 0.537 & $\begin{array}{l}17 \overline{25} .0 \\
\overline{-} \\
33 \overline{79} .0\end{array}$ & $\begin{array}{r}-\overline{-} \\
96.77 \\
3.48 \\
\text { Bruch }\end{array}$ \\
\hline $\begin{array}{c}\text { Stück von } 8 \text { zu } 9 \\
\text { (Fette) } \ldots\end{array}$ & 4 & 9.000 & 10.420 & 223.4 & 240.4 & 347.00 & 0.620 & $\begin{array}{l}17 \overline{25} .0 \\
\overline{-} \overline{25} .0 \\
\overline{-} \\
2939.0\end{array}$ & $\begin{array}{r}- \\
99.02 \\
0.89 \\
151.56 \\
6.44 \\
\text { Bruci }\end{array}$ \\
\hline $\begin{array}{c}\text { Stück von } 8 \text { zu } 9 \\
(\text { Fette) } .\end{array}$ & 10 & 9.000 & 10.540 & 225.5 & 243.0 & 281.25 & 0.487 & $\begin{array}{l}\overline{17 \overline{44} .0} \\
\overline{4} \overline{24} .0\end{array}$ & $\begin{array}{r}-\overline{97.04} \\
1.55 \\
\text { Bruch }\end{array}$ \\
\hline $\begin{array}{c}\text { Stück von } 9 \text { zu } 10 \\
\text { (Fette) } \ldots\end{array}$ & 11 & 11.000 & 13.000 & 254.6 & 283.5 & 475.00 & 0.506 & $\begin{array}{l}17 \overline{44} .0 \\
\overline{-} \overline{44.0} \\
42 \overline{4} .0 \\
53 \overline{9} .0\end{array}$ & $\begin{array}{r}- \\
85.48 \\
0.51 \\
171.40 \\
7.88 \\
240.89 \\
22.99 \\
\text { Bruch }\end{array}$ \\
\hline $\begin{array}{c}\text { Stück von } 11 \text { zu } 12 \\
\text { (recharge) }\end{array}$ & 7 & 13.000 & 14.000 & 289.9 & 324.1 & 697.00 & 0.530 & $\begin{array}{r}1 \overline{4} \overline{4} .0 \\
\overline{-} \\
2544.0 \\
3344.0 \\
39 \overline{4} 0 \\
\overline{-} \\
49 \overline{4} .0 \\
\overline{-} \\
5944.0 \\
6404.0\end{array}$ & $\begin{array}{r}- \\
86.63 \\
5.11 \\
137.84 \\
176.26 \\
11.10 \\
210.54 \\
18.78 \\
281.82 \\
28.89 \\
421.47 \\
\text { Bruch }\end{array}$ \\
\hline
\end{tabular}




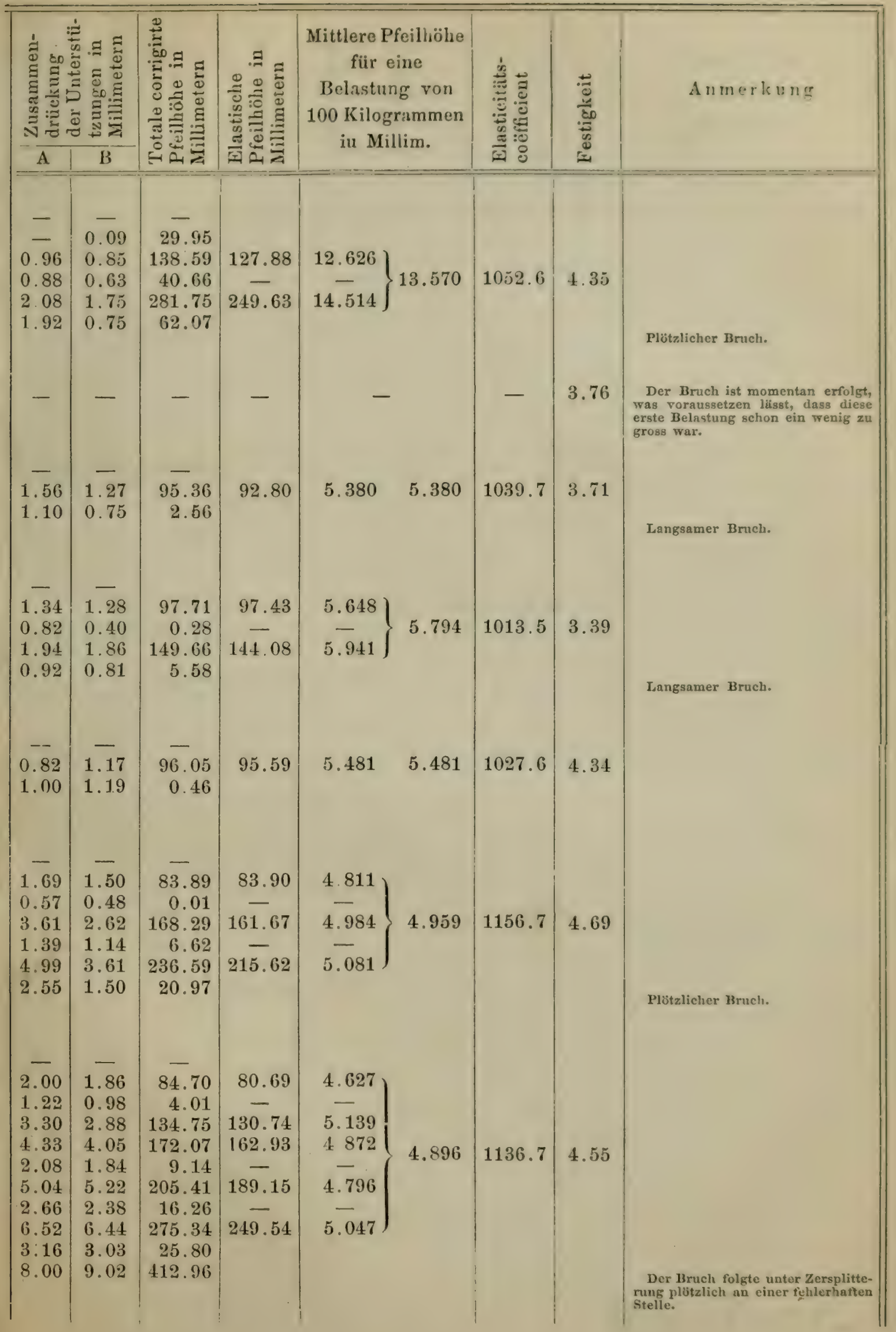




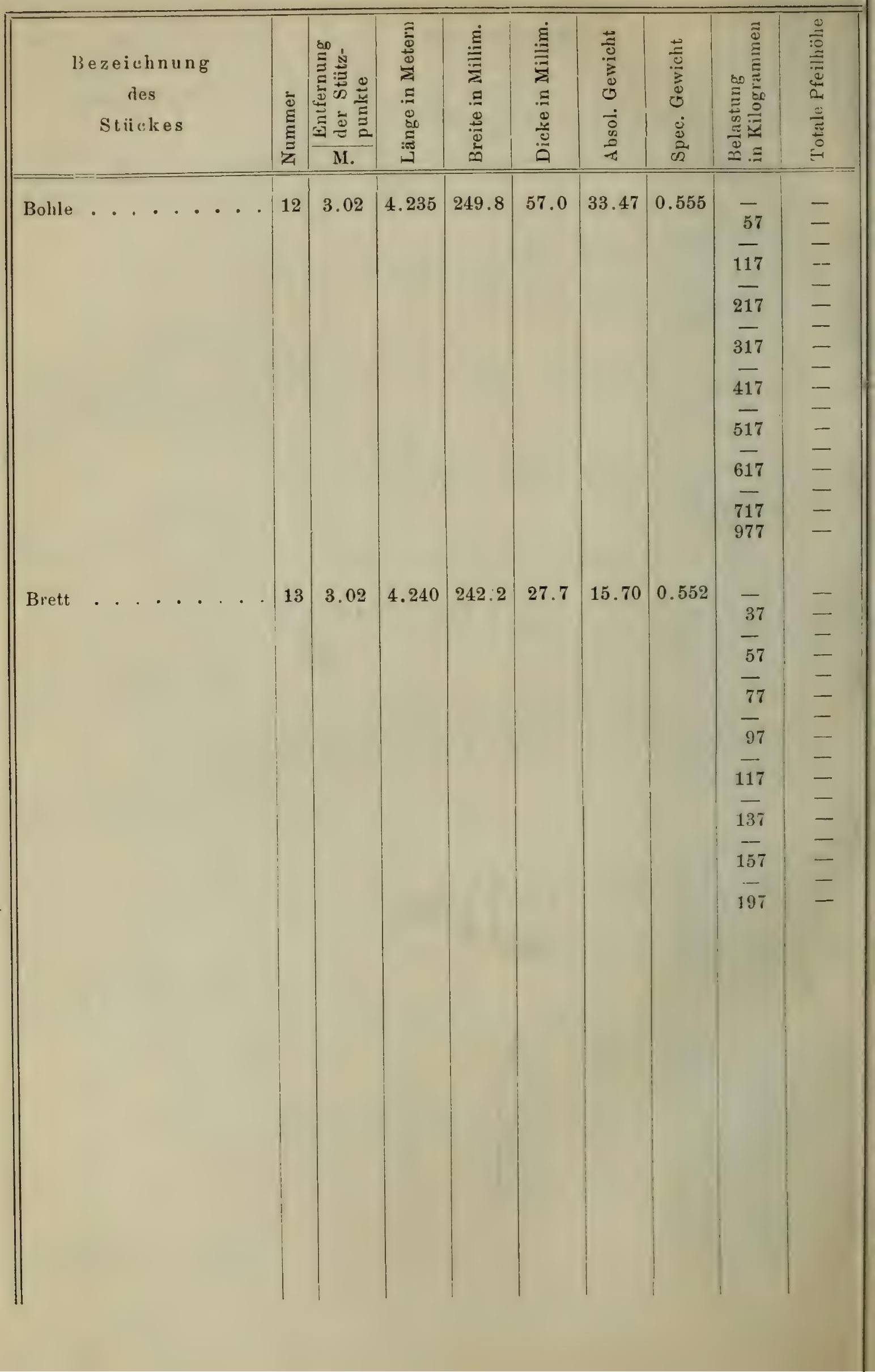




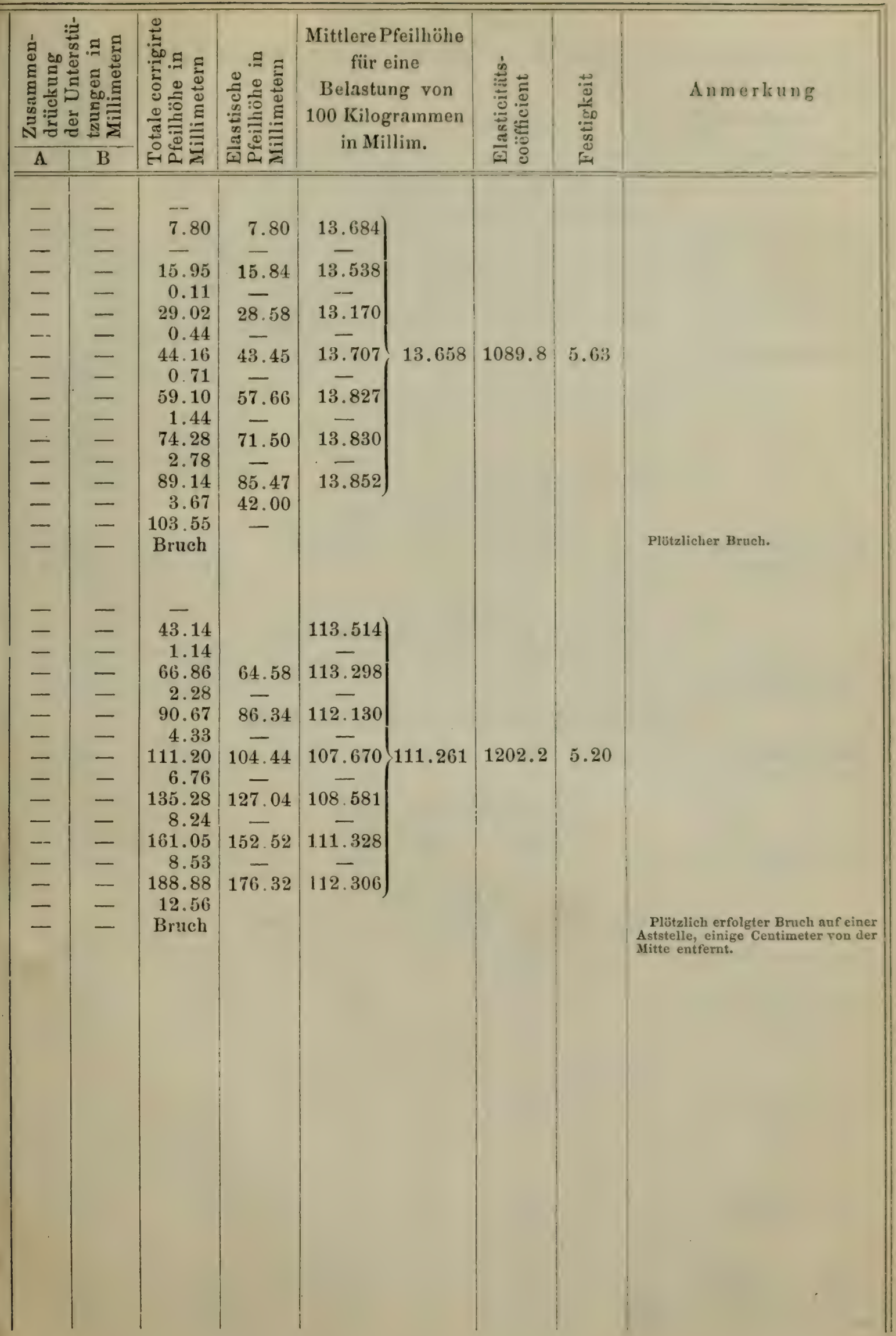




\section{Tabelle Nr. XIX.}

Einfluss des Alters der Exposition und Bodenbeschaffenheit.

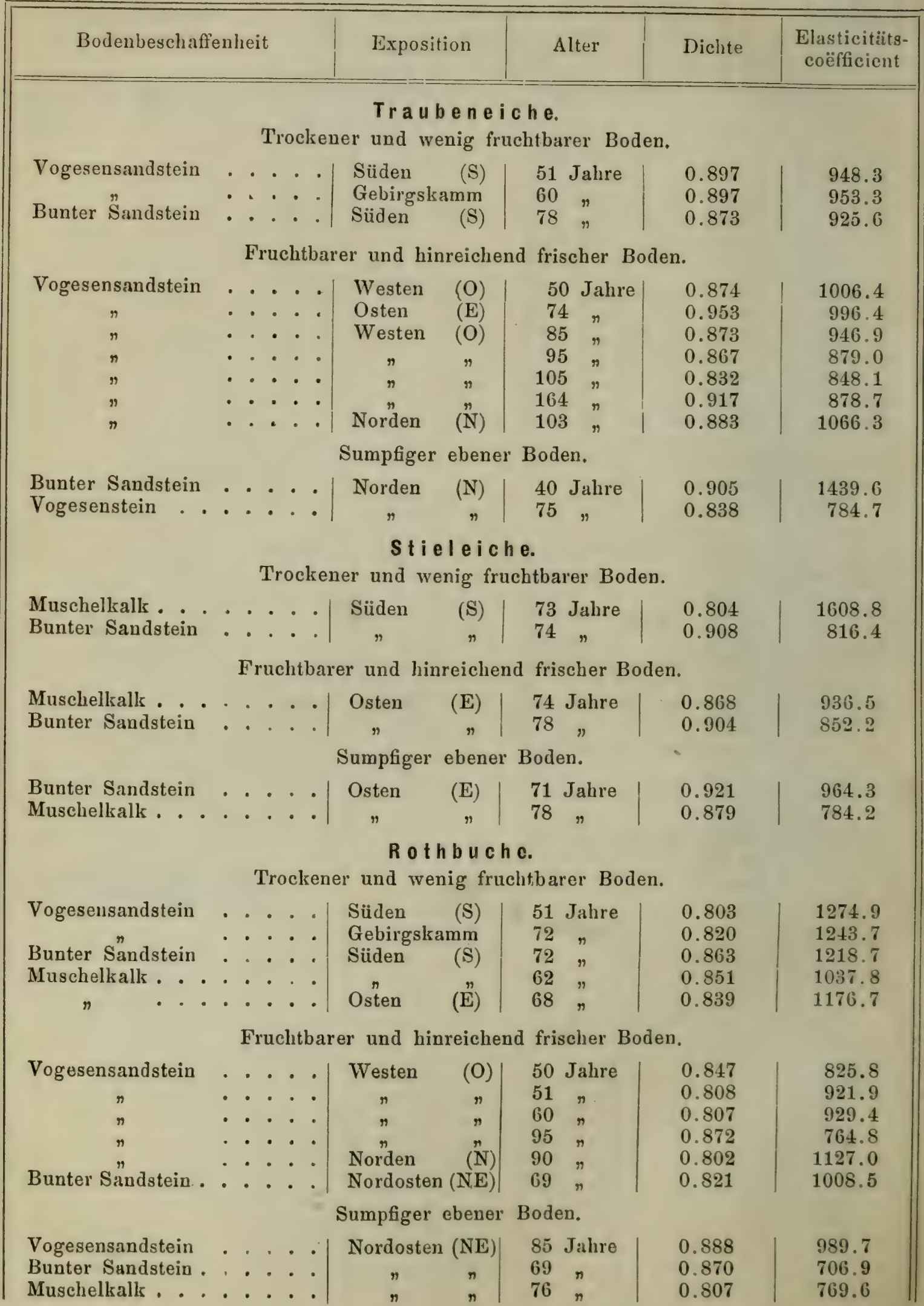


- $123-$

Tanne. (Vogesensandstein.)

\begin{tabular}{|c|c|c|c|c|c|c|c|c|c|}
\hline \multirow{2}{*}{$\begin{array}{l}\text { Bezeichnung } \\
\text { des } \\
\text { Stückes }\end{array}$} & \multicolumn{3}{|c|}{$\begin{array}{l}\text { Trockenor Bodon } \\
\text { gegen Süden }\end{array}$} & \multicolumn{3}{|c|}{$\begin{array}{l}\text { Fruehtharer Boden } \\
\text { gegen Norden }\end{array}$} & \multicolumn{3}{|c|}{$\begin{array}{l}\text { Fruchtluarer Boden } \\
\text { gegen NW. } \\
\text { (aber ein wenig trocken) }\end{array}$} \\
\hline & Dichte & $\begin{array}{l}\text { Elastici- } \\
\text { tiitscoèt }\end{array}$ & $\begin{array}{l}\text { Festig- } \\
\text { keit }\end{array}$ & llichte & $\begin{array}{l}\text { Elastici- } \\
\text { titaconf. }\end{array}$ & $\begin{array}{l}\text { Festig- } \\
\text { kerit }\end{array}$ & Dichte & $\begin{array}{l}\text { Elastici- } \\
\text { tätscouif. }\end{array}$ & $\begin{array}{l}\text { Festig- } \\
\text { keit }\end{array}$ \\
\hline Sparren . . & 0.485 & 1115.6 & 5.45 & 0.454 & 1272.4 & 4.82 & 0.50 .3 & 1384.5 & 6.19 \\
\hline Stück v. $6 \% .7$ & 0.573 & - & 3.76 & 0.491 & 1052.6 & 4.35 & 0.510 & 1437.5 & 5.84 \\
\hline 8,9 & 0.537 & 1039.7 & 3.71 & 0.620 & 1013.5 & 3.39 & 0.487 & 1027.6 & 4.34 \\
\hline 9,10 & - & - & 一 & - & - & - & 0.506 & 1156.7 & 4.69 \\
\hline$n \quad 11,12$ & - & - & - & - & - & - & () 530 & 1136.7 & 4.55 \\
\hline
\end{tabular}





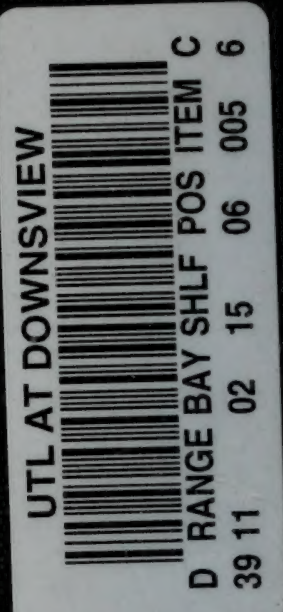

